\title{
Cognitive adaptations in two sympatric mouse lemur species occupying different ecological niches
}

\author{
Dissertation
}

\author{
for the award of the degree \\ "Doctor rerum naturalium" (Dr. rer. nat.) \\ of the Georg-August-University Göttingen
}

within the doctoral program Behavior and Cognition (BeCog) of the Georg-August-University School of Science (GAUSS)

submitted by

\section{Johanna Henke-von der Malsburg}

from Gräfelfing, Germany

Göttingen, 2021 


\section{Thesis committee}

Dr. Claudia Fichtel

Behavioral Ecology and Sociobiology Unit, German Primate Centre, Leibniz Institute for Primate Research, Göttingen

Prof. Dr. Julia Ostner

Department of Behavioral Ecology, Johann-Friedrich-Blumenbach Institute of Zoology and Anthropology, Georg-August-University Göttingen \& Research Group Social Evolution in Primates, German Primate Centre, Leibniz Institute for Primate Research, Göttingen

Prof. Dr. Lars Penke,

Biological Personality Psychology, Georg-Elias-Müller Institute of Psychology, GeorgAugust-University Göttingen

\section{Members of the examination board}

Reviewer: $\quad$ Dr. Claudia Fichtel

Second reviewer: Prof. Dr. Julia Ostner

\section{Further members of the examination board}

Prof. Dr. Peter M. Kappeler

Department of Sociobiology/Anthropology, Johann-Friedrich-Blumenbach Institute of Zoology and Anthropology, Georg-August-University Göttingen \& Behavioral Ecology and Sociobiology Unit, German Primate Centre, Leibniz Institute for Primate Research, Göttingen

Prof. Dr. Stefan Scheu

Animal Ecology Working Group, Johann-Friedrich-Blumenbach Institute of Zoology and Anthropology, Georg-August-University Göttingen Germany

Prof. Dr. Katja Liebal

Human Biology \& Primate Cognition, Institute of Biology, Leipzig University, Germany 


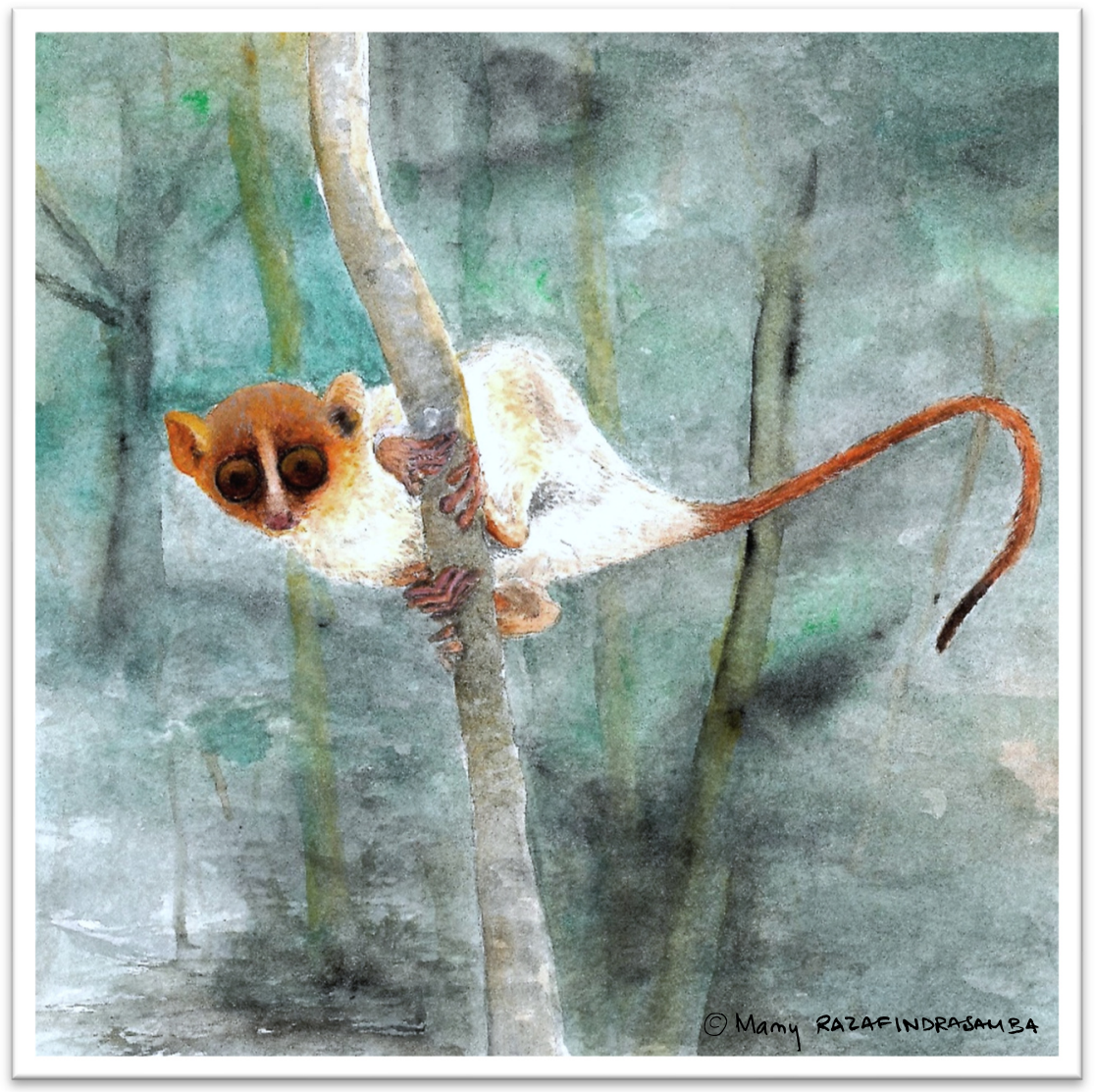





\section{Table of contents}

Table of contents

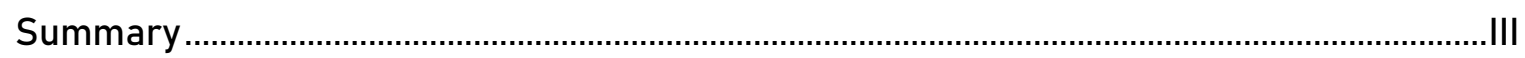

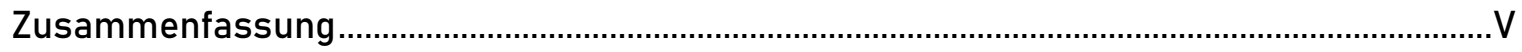

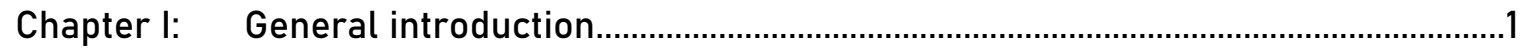

1 Towards understanding variation in brain size and cognitive abilities..............2

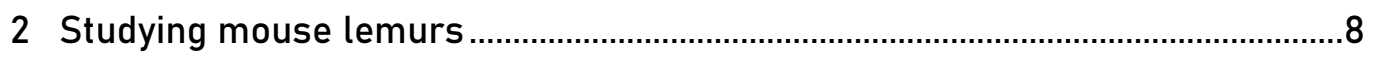

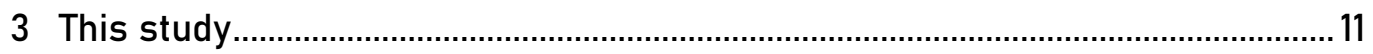

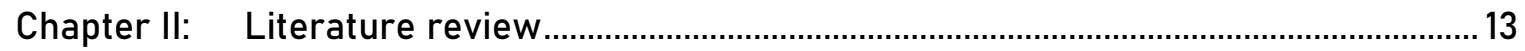

Linking ecology and cognition: does ecological specialization predict cognitive test

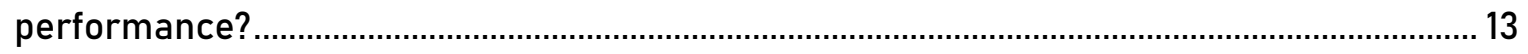

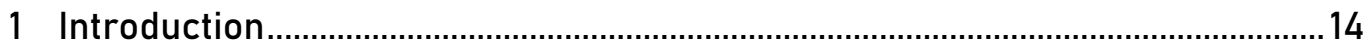

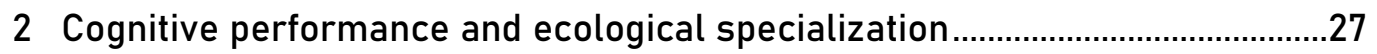

3 Ecological generalism versus ecological specialization...................................... 31

4 Variation in cognitive performance in relation to group size ............................ 44

5 Variation in cognitive performance in relation to brain size ............................. 45

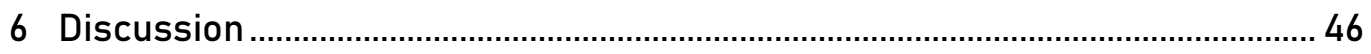

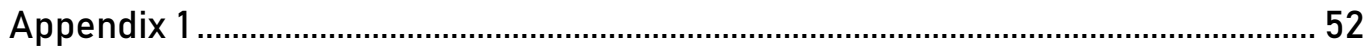

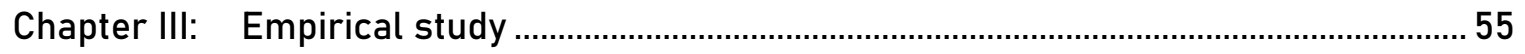

Linking cognition to ecology in wild sympatric mouse lemur species................................. 55

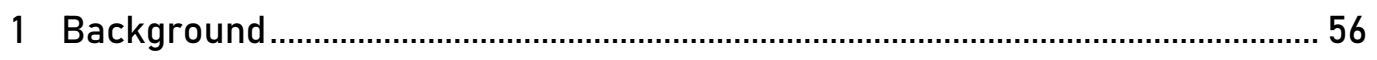

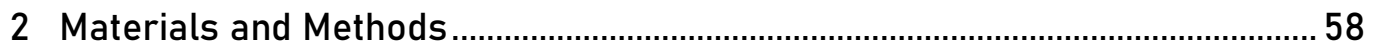

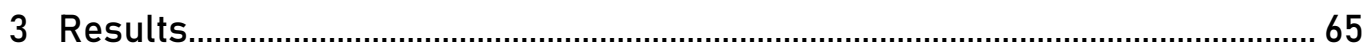

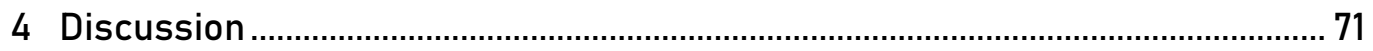

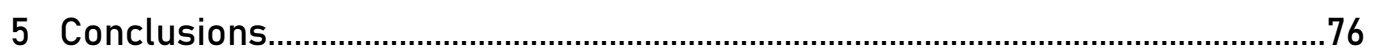

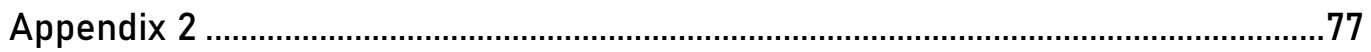

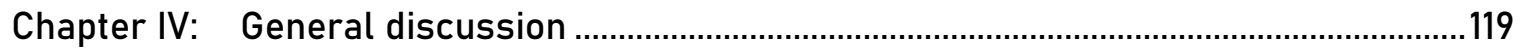

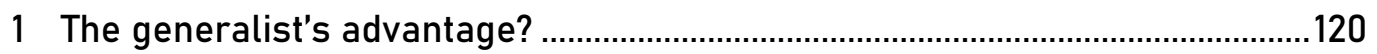

2 Coevolution with brain size and fitness implications ....................................... 127

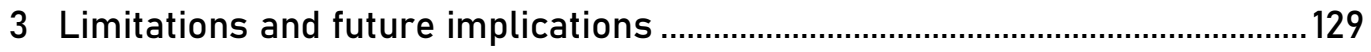

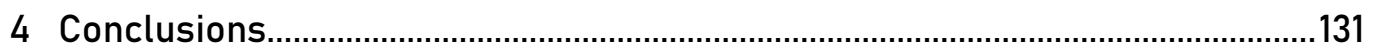

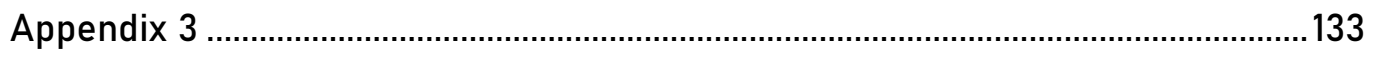

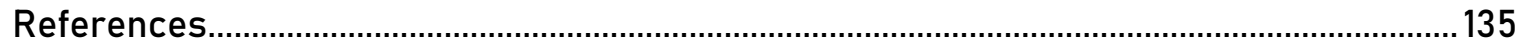

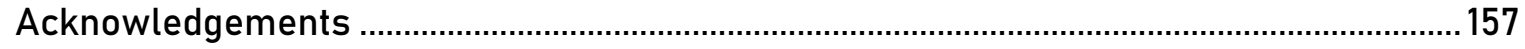

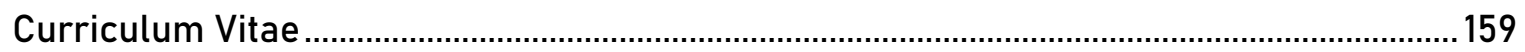

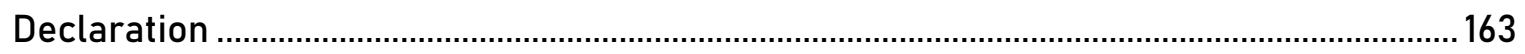




\section{Summary}

Cognition describes an individual's abilities to perceive, process, and act on information of the abiotic and biotic environment. The investigation of cognitive variation between individuals and species has been of interest for many decades and several hypotheses have been proposed to explain the evolution of cognition. Sizes of certain brain areas covary with the cognitive abilities they process, and socio-ecological factors have been related to various brain size measures. However, covariations of phenotypic cognitive performances with socio-ecological factors are essential to understand the adaptive value of cognitive abilities. While links with social factors have been the focus of comparative studies, which and how cognitive abilities and ecological factors link with each other, has been less systematically investigated. Precisely, it is assumed, that ecological generalists consistently outperform ecological specialists in cognitive challenges, but a respective link lacks systematic empirical evidence.

In my first theoretic approach, I reviewed the literature for comparative studies that experimentally assessed cognitive performances among at least two species of the same phylogenetic order. I found that cognitive variation can be related to species-specific dietary preferences, related foraging behaviors, migratory behavior, or habitat complexity. However, other ecological factors, such as the synecology with predators or parasites, or the degree of ecological specialization, have largely been disregarded to be linked with cognitive performances. Only a subset of the reviewed studies specifically mentioned the degree of ecological specialization of the species in comparison. After complementing the respective information using additional literature, I applied a sign-test to estimate whether the degree of dietary or habitat specialization consistently covaries with cognitive performances. Across a total of 34 comparisons, $\mathrm{N}=26$ cognitive performances differed between the species in comparison. In $62 \%$ of these comparisons, the dietary generalist achieved higher performance scores than the relative dietary specialist. Habitat generalists achieved higher scores than relative habitat specialists in $72 \%$ of the comparisons. However, ecological generalism was not significantly associated with higher performances that go beyond innovative and flexible learning abilities. It 
remains therefore questionable, whether generalist species consistently outperform more specialist species across a broader range of cognitive abilities.

In my second empirical approach, I applied a conclusive cognitive test battery to two wild primate sister species. The gray and the Madame Berthe's mouse lemur possess a comparable social system, occur in sympatry at the chosen study site, but are differentially adapted to ecological factors. Essentially, the gray mouse lemur is a dietary and habitat generalist, while the Madame Berthe's mouse lemur is more specialized along these ecological niche axes. Occupying different ecological niches, while experiencing similar ecological challenges, assumes complex evolutionary processes for ecological adaptations that may be linked to cortical development, and may covary with cognition. Following the Opportunistic Intelligence Hypothesis, the generalist gray mouse lemur should consistently outperform the specialist Madame Berthe's mouse lemur across the various cognitive tests. Alternatively, species-specific performance levels could covary with specific ecological adaptations. For instance, gray mouse lemurs feed more on gum, a characteristic that has been associated with better self-control. Madame Berthe's mouse lemurs have larger home ranges and feed mostly on spatially clumped homopteran secretions, which may covary with spatial abilities. In summary, the two species differed in about two third of the assessed performance scores. Gray mouse lemurs were more innovative, which may provide them the advantage to adaptively respond to variable environmental conditions. However, they did not consistently outperform Madame Berthe's mouse lemurs, which were more active and learned visual and spatial reward contingencies faster.

Hence, the experimental results in mouse lemurs parallel the theoretic findings of the reviewed literature, in that generalist species do not outperform specialist species per se. Despite the apparent superposition of generalists with respect to innovative problemsolving, they do not consistently outperform specialists in other cognitive tasks. Rather, performances are better linked to other ecological factors, which might be more accurately disentangled in future studies using metric estimates instead of the categoric classification of ecological specialization. Nevertheless, as the first large scale comparative approach within this exciting research field, the results of my thesis offer substantial insight into the link between cognitive test performances and ecological factors. 


\section{Zusammenfassung}

Mit kognitiven Fähigkeiten können Tiere Informationen aus der abiotischen und biotischen Umwelt wahrnehmen, verarbeiten und auf diese Informationen in einem angemessenen Umfang reagieren. Die Untersuchung kognitiver Variabilität zwischen Individuen und Arten ist von großem wissenschaftlichem Interesse, das zur Formulierung mehrerer Hypothesen zur Erklärung kognitiver Evolution geführt hat. Hirnareale können in Größe und Entwicklung mit kognitiven Fähigkeiten, die sie verarbeiten korrelieren, und sozio-ökologische Faktoren wurden mit verschiedenen Maßen zur kognitiven Entwicklung in Verbindung gebracht. Um den adaptiven Wert kognitiver Fähigkeiten zu verstehen, sind jedoch Kovariationen zwischen phänotypischen kognitiven Leistungen und sozio-ökologischen Faktoren ausschlaggebend. Während die Untersuchung entsprechender Zusammenhänge mit sozialen Faktoren im Fokus vergleichender Studien standen, wurde weniger systematisch untersucht, inwiefern kognitive Fähigkeiten mit welchen ökologischen Faktoren verknüpft sind. So wird zwar vermutet, dass ökologische Generalisten ökologische Spezialisten bei kognitiven Herausforderungen konsequent übertreffen, ein entsprechender Zusammenhang ist jedoch empirisch nicht systematisch belegt.

In dem ersten theoretischen Ansatz meiner Dissertationsarbeit, habe ich bisherige vergleichende Studien untersucht, in denen kognitive Leistungen zwischen mindestens zwei Arten der gleichen phylogenetischen Ordnung experimentell verglichen wurden. Diese Studien belegen, dass kognitive Variationen mit artspezifischen Nahrungspräferenzen, damit im Zusammenhang stehenden Futtersuchverhalten, sowie der Anpassung zur Migration oder der Komplexität des Lebensraums zusammenhängen können. Andere ökologische Faktoren, wie beispielsweise eine Synökologie mit Raubtieren oder Parasiten, oder der Grad der ökologischen Spezialisierung, wurden jedoch nur gelegentlich mit kognitiven Leistungen in Verbindung gebracht. Nur ein Teil der untersuchten Studien erwähnte ausdrücklich den Grad der ökologischen Spezialisierung der verglichenen Arten. Nachdem ich die entsprechenden Informationen durch das Heranziehen zusätzlicher Literatur ergänzt hatte, verwendete ich einen Vorzeichentest, um abzuschätzen, ob der Grad der Nahrungs- oder 


\section{Zusammenfassung}

Habitatspezialisierung konsistent mit kognitiven Leistungen kovariiert. In insgesamt 26 von 34 Vergleichen unterschieden sich die untersuchten Arten in ihren kognitiven Leistungen. In $62 \%$ dieser Vergleiche erzielte der Nahrungsgeneralist höhere Leistungswerte als der relative Nahrungsspezialist. Habitatgeneralisten erzielten in $72 \%$ der Vergleiche höhere Werte als relative Habitatspezialisten. Allerdings war die Ausprägung zum ökologischen Generalisten nicht signifikant mit höheren Leistungswerten verbunden, vor allem, wenn andere kognitive Fähigkeiten als innovative und flexible Lernfähigkeiten untersucht wurden. Es bleibt daher fraglich, ob Generalisten über ein breiteres Spektrum kognitiver Fähigkeiten hinweg generell besser abschneiden als spezialisiertere Arten.

In dem zweiten empirischen Ansatz meiner Dissertationsarbeit, habe ich zwei wildlebende Primatenarten in einer umfangreichen kognitiven Testbatterie getestet. Die beiden Schwesterarten, der Graue und der Madame Berthe-Mausmaki besitzen ein vergleichbares Sozialsystem, sind im gewählten Studienort zeitgleich vorzufinden, sind dort aber unterschiedlich an ökologische Faktoren angepasst. Im Wesentlichen ist der Graue Mausmaki ein Nahrungs- und Habitatgeneralist, während der Madame BertheMausmaki entlang dieser Achsen ihrer ökologischen Nische stärker spezialisiert ist. Das Besetzen unterschiedlicher ökologischer Nischen bei ähnlichen ökologischen Herausforderungen steht mit komplexen evolutionären Prozessen für ökologische Anpassungen in Zusammenhang, die mit der kortikalen Entwicklung verbunden sein und mit kognitiven Fähigkeiten kovariieren können. Entsprechend der Hypothese zur Opportunistischen Intelligenz, sollte der generalistische Graue Mausmaki den spezialisierten Madame Berthe-Mausmaki durchwegs in kognitiven Tests übertreffen. Alternativ dazu könnten artspezifische Leistungsniveaus mit artspezifischen ökologischen Anpassungen kovariieren. Zum Beispiel fressen Graue Mausmakis mehr Baumharz, eine Eigenschaft, die mit besserer Selbstkontrolle in Verbindung gebracht wurde. Madame Berthe-Mausmakis bewegen sich über größere Aktionsräume und ernähren sich hauptsächlich von ungleichmäßig verteilten Insektensekreten, was mit räumlichen Fähigkeiten kovariieren könnte. Letztendlich haben sich die beiden Arten in etwa zwei Dritteln der untersuchen kognitiven Leistungswerte unterschieden. Graue Mausmakis waren dabei innovativer, was ihnen den Vorteil verschaffen könnte, adaptiv 
auf variable Umweltbedingungen zu reagieren. Jedoch übertrafen sie die Madame BertheMausmakis nicht durchgehend. Diese waren beispielsweise aktiver und konnten visuelle und räumliche Zusammenhänge mit einer Futterbelohnung schneller erlernen.

Meine experimentellen Ergebnisse der Mausmakis decken sich also mit den theoretischen Erkenntnissen der untersuchen Literatur, indem Generalisten Spezialisten nicht per se in kognitiven Experimenten übertreffen. Trotz der scheinbaren Überlegenheit von Generalisten in Bezug auf innovatives Problemlösen, sind sie Spezialisten in anderen kognitiven Aufgaben nicht durchweg überlegen. Vielmehr sind kognitive Leistungen besser mit anderen ökologischen Faktoren verknüpft, die in zukünftigen Studien detaillierter entschlüsselt werden könnten, wenn anstelle der kategorischen Klassifizierung der ökologischen Nische eher metrische Variablen gemessen würden. Nichtsdestotrotz geben die Ergebnisse meiner Arbeit als erster groß angelegter vergleichender Ansatz in diesem spannenden Forschungsfeld einen wesentlichen Einblick in den Zusammenhang zwischen testbaren kognitiven Fähigkeiten und ökologischen Faktoren. 


\section{Chapter I: General introduction}

Cognition - "The word seems straightforward, yet it is often a cause of debate in the psychological and neuroscience fields, particularly about whether a behavior of an animal that happens not to be human is truly "cognitive", in a similar sense to human cognition." - Geoffrey North (in Bayne et al. 2019, p R608)

In non-human animals, we consider cognition as all cognitive traits, that provide an individual the abilities to "acquire, process, store and act on information from the environment" (Shettleworth 2009). Cognitive traits vary between individuals, as well as between species. Due to the neurological nature of cognition, it is assumed that cognitive abilities covary with neuronal structures, such as the development of certain brain areas. Consequently, the Social Intelligence Hypothesis (Humphrey 1976; Whiten and Byrne 1988) and the Ecological Intelligence Hypothesis (Parker and Gibson 1977; Milton 1988) suggest that brain size varies with social or ecological factors. While these hypotheses have been posited to be exclusive, more recent studies support a rather complementary influence of social and ecological factors on cognitive evolution (Emery and Clayton 2004; Lefebvre and Sol 2008; Reader et al. 2011; MacLean et al. 2012; Rosati 2017a). A respective link between social complexity and cognitive abilities finds broad support (Bond et al. 2003; Bond et al. 2007; MacLean et al. 2008; Amici et al. 2008; Byrne and Bates 2010; MacLean et al. 2013; Perry et al. 2017; Ashton et al. 2018b; Amici et al. 2018). However, which specific ecological factors covary with which cognitive abilities has rarely been empirically investigated across the animal kingdom.

To better understand the ecological nature of cognitive variation and, therefore, the adaptive value of certain cognitive abilities, potential links between ecological factors and cognitive abilities need to be systematically investigated. By comparing individuals of different species that exhibit different ecological adaptations while possessing comparable social organizations, the coevolution of cognitive traits and adaptations to ecological conditions may be better understood. With my thesis, I contribute to this endeavor by investigating cognitive variation among two closely related and sympatric primate species that are differently adapted to ecological conditions but are socially similarly organized. 
In the following, I explain the principles of cognitive variation in response to socioecological factors. Thereby, I wrap-up the most prominent hypotheses that have been proposed to understand the evolution of brain size and correlating cognitive abilities. Further, I provide a brief overview of common links, as well as mixed patterns of previous investigations on cognitive variation in relation to socio-ecological factors. Subsequently, I describe the concept of niche theory with the classification of ecological specialization and respective adaptive responses to habitat and dietary breadth. Then, I introduce two mouse lemur species which I tested for this thesis by also giving an overview of prior investigations in this genus. Finally, I outline the specific aims of my thesis and provide an overview of the content of the next chapters.

\section{Towards understanding variation in brain size and cognitive abilities}

As Thorndike emphasized in the $18^{\text {th }}$ century, it is "[associations], and not instinct, [that] make the animal use the best feeding grounds, sleep in the same lair, avoid new dangers and profit by new changes in nature" (Thorndike 1898). An essential prerequisite for associative learning abilities, as for cognitive traits in general, are neuronal structures. However, the more complex the neuronal structures and the larger the metabolically expensive brain, the more energy must be consumed (Rumbaugh and Washburn 2003; Roth and Dicke 2005). Therefore, larger brains should only evolve when their benefits exceed their high metabolic costs (Laughlin et al. 1998; Lennie 2003). Essentially, such benefits can be related to an animal's socio-ecological conditions. Hence, different hypotheses have been proposed to explain variation in brain size and subsequently variation in cognitive abilities.

\subsection{Principles of cognitive variation in response to socio-ecological factors}

According to the Social Intelligence Hypothesis (SIH), it is suggested, that brain size covaries with social factors (Humphrey 1976; Whiten and Byrne 1988; Dunbar 1998; Dunbar et al. 
2007). Specifically, social complexity seems to be associated with brain size (Jolly 1966; Barton 1996; Kudo and Dunbar 2001; Byrne and Bates 2010). More socially complex species are those that live in larger groups instead of smaller pair-bonds or that spend most of their active time solitary. Subsequently, they experience more complex social challenges that require specific social skills, like managing group dynamics, or effectively communicating and coordinating with group members (Dunbar et al. 2007; Kappeler 2019; Peckre et al. 2019). In support for the SIH, primates, bats, and specifically insectivores that live in larger groups developed larger brains than species living in smaller groups (Barton 1996; Dunbar 1998; Kudo and Dunbar 2001; Byrne and Bates 2010; MacLean et al. 2013; Todorov et al. 2019). However, this correlation is debated in carnivores and ungulates (Shultz and Dunbar 2006; Dunbar and Shultz 2007; Shultz and Dunbar 2007; Holekamp et al. 2007; Pérez-Barbería et al. 2007; Finarelli and Flynn 2009), and seems to be absent in birds (Beauchamp and Fernández-Juricic 2004; Iwaniuk and Arnold 2004). Additionally, recent comparative analyses indicate that brain size is better predicted by ecological than social factors (DeCasien et al. 2017; Powell et al. 2017; DeCasien and Higham 2019).

Following the Ecological Intelligence Hypothesis (EIH), larger brains allow animals to develop better cognitive abilities to cope with ecological challenges (Parker and Gibson 1977; Milton 1988). Evidence for the EIH has been reported in many animals, including birds and primates (Harvey et al. 1980; MacLean et al. 2009; DeCasien et al. 2017; Powell et al. 2017). Specifically, birds that rely on food-caching or food-hoarding develop larger hippocampi, the brain area which is associated with spatial processing and memory (Krebs 1990; Sherry et al. 1992; Clayton 1998). This relation parallels with spatial abilities in mammals, with species feeding on spatiotemporally distributed fruits having larger brains than more folivorous species (Clutton-Brock and Harvey 1980; MacLean et al. 2009; Mace et al. 2009; Rosati et al. 2014). In primates, specific manipulation skills, such as extractive foraging techniques, covary with neocortex size (Heldstab et al. 2016). Nocturnal or cathemeral lemurs have larger relative brain sizes than diurnal species (MacLean et al. 2009).

Additionally, the Cognitive Buffer Hypothesis (Deaner et al. 2003) posits that a larger brain specifically buffers challenges that come along with variable environmental conditions, as it allows for more adaptive behavioral flexibility. Therefore, generalists, 
Chapter I: General introduction

which face more variable conditions, might have evolved larger brains than more specialized species (Reader 2003; Lefebvre et al. 2004; Sol et al. 2005b; Ducatez et al. 2015).

\section{2 (Co-)Variation of cognitive abilities}

Despite an apparent covariation of cognitive abilities with brain size or brain structure (Clutton-Brock and Harvey 1980; Barton and Harvey 2000; Kudo and Dunbar 2001; Deaner et al. 2007; Chittka and Niven 2009; Dicke and Roth 2016), the suggested hypotheses do not consider a direct relationship of social or ecological factors with cognitive abilities. However, when investigating variation in cognitive abilities in animals, we mostly rely on the behavioral phenotype that can be observed while solving problems (Shettleworth 2009). Thus, cognitive abilities have been operationalized using performance scores in artificial experimental set-ups (Ramsey et al. 2007; Healy et al. 2009; Pritchard et al. 2016; Shaw and Schmelz 2017).

Yet, such empirical studies report evidence for a covariation with social, as well as ecological factors and a link with the size of specific cortical areas. For instance, social complexity correlated positively with performance scores of inhibitory control, transitive inference, or flexible learning abilities in primates and birds (Bond et al. 2003; Bond et al. 2007; MacLean et al. 2008; Amici et al. 2008; Ashton et al. 2018a; Amici et al. 2018; Miller et al. 2019; Ashton et al. 2019). Inhibitory control is also enhanced in more frugivorous or gum-feeding primates, as compared to rather folivorous primates or primates that predominantly feed on mobile insects, and inhibitory control further correlates with brain size (Stevens et al. 2005; MacLean et al. 2014). Migratory or food-storing birds and mammals experience better spatial abilities than residential or non-storing species and develop larger hippocampi (Shettleworth et al. 1990; Clayton and Krebs 1994a; Cristol et al. 2003; Emery and Clayton 2004; Barkley and Jacobs 2007). Spatial abilities are also enhanced in species living in more complex habitats, or species that feed on more stable rather than mobile food items (Pleskacheva et al. 2000; White and Brown 2015a; Trapanese et al. 2019; Teichroeb and Vining 2019). More actively foraging species are more flexible learners than sit-and-wait predators (Day et al. 1999a). Flexible learning and related innovative propensities are also enhanced in species or individuals, that experience 
harsher environmental conditions, more urban environments or seasonal changes in food availability (van Woerden et al. 2012; Tebbich et al. 2016; Sayol et al. 2016; Preiszner et al. 2017; Chow et al. 2021). Further, learning abilities have been related to both ecological factors and brain morphology in fish (White and Brown 2015a; White and Brown 2015b; Pike et al. 2018). However, some studies report no difference in cognitive performances in relation to socio-ecological factors or brain size measures (Healy and Suhonen 1996; Bond et al. 2007; Clarin et al. 2013; Hernández-Montero et al. 2020; Pladevall et al. 2020).

Additionally, it is questioned if cognitive abilities emerge in a convergent manner or rather in concordance with a mosaic brain evolution (Barton and Harvey 2000). Functionally different brain areas vary in size in relation to cognitive and sensory specializations to respective ecological adaptations and, therefore, cognitive abilities would vary between cognitive domains (Cosmides and Tooby 1994; Domain-Specific Hypothesis; Barton 1996; Striedter 2006). For example, olfactory cortical structures are larger in nocturnal than in diurnal primates, which have larger visual cortexes (Barton et al. 1995). Further, both of these brain regions are enlarged in primates with high-quality diets or group-living species, while brain regions that are related to spatial processing are enlarged in primates with low-quality diets or solitary species (DeCasien and Higham 2019).

Alternatively, according to the Domain-General Hypothesis or the congruent General Intelligence Hypothesis, cognitive abilities covary across cognitive domains and correlate onto a common psychometric g-factor (Spearman 1904; Humphreys 1979; Jensen 1985; Deaner et al. 2006). In WEIRD (western, educated, industrialized, rich, and democratic) people, a higher-level $g$-factor even provides advantages in several decision-making processes (Gottfredson 1997; Henrich et al. 2010). In non-human animals, a $g$-factor has been reported for rodents, dogs, birds, and non-human primates (Anderson 1993; Herndon et al. 1997; Galsworthy et al. 2002; Matzel et al. 2003; Banerjee et al. 2009; Keagy et al. 2011; Reader et al. 2011; Isden et al. 2013; Hopkins et al. 2014; Matzel et al. 2011; Woodley of Menie et al. 2015; Shaw et al. 2015; Arden and Adams 2016; Ashton et al. 2018a; Damerius et al. 2019). However, other studies on rodents, birds, as well as non-human primates reject the Domain-General Hypothesis (Locurto et al. 2003; Locurto et al. 2006; 
Herrmann et al. 2007; Boogert et al. 2011; Herrmann and Call 2012; Amici et al. 2012; Anderson et al. 2017; Huebner et al. 2018).

Therefore, common patterns of (co)variation of cognitive abilities are not yet completely understood across the animal kingdom. Cognitive abilities may correlate within, and partly also between cognitive domains. Further, cognitive abilities can covary with certain social, as well as ecological factors. The investigation of a respective link with sociality has received great interest in previous studies (Bond et al. 2003; Bond et al. 2007; MacLean et al. 2008; Amici et al. 2008; Byrne and Bates 2010; Ashton et al. 2018b; Amici et al. 2018). The link between cognitive variation and ecological factors remains biased towards innovative and spatial abilities, and towards a species diet, invasion potential, or migratory behavior (Pleskacheva et al. 2000; Day et al. 2003; Cristol et al. 2003; Stevens et al. 2005; Bond et al. 2007; MacLean et al. 2009; White and Brown 2015a; Rosati 2017b; Trapanese et al. 2019; Teichroeb and Vining 2019). Specifically, a covariation between cognitive abilities and the degree of ecological specialization has been rarely investigated.

\subsection{Niche theory and the classification of ecological specialization}

The degree of ecological specialization aims to characterize an animal's ecological niche, i.e., a multidimensional space comprising all ecological factors to which the animal has evolved adaptive phenotypic traits. Species that experience a broad habitat or dietary breadth are classified as ecological generalists, while species with more specialized preferences are classified as ecological specialists (Hutchinson 1953; MacArthur 1957; Hutchinson 1957; Roughgarden 1972; Sargeant 2007). Adaptive phenotypic traits can be either specific morphological structures or behavioral tactics to explore and exploit specific habitats and food patches most efficiently, or more general and flexible behaviors to be able to explore and exploit various habitats and food types. For example, nectivorous hummingbirds (Trochilidae) or bats (e.g., Leptonycteris spp.) have evolved specialized rostra, such as elongated and thinner beaks or tongues and snouts, with which they can more efficiently exploit flowers that are specifically rich in nectar (Hoffmeister 1957; Temeles et al. 2002; Rico-Guevara et al. 2019). In line with these morphological adaptations, these species have evolved behavioral adaptations which allows them to 
better profit from their specialized lifestyle. Nectivorous Saussure's long-nosed bats (L. yerbabuenae), for example, have more pronounced spatial skills compared to Palla's long-tongued bats (Glossophaga soricine), which additionally feed on fruits and insects (Henry and Stoner 2011). In contrast, generalists do not possess such specialized adaptations, but are more likely to innovate and they are more flexible learners than specialists (Daly et al. 1982; Overington et al. 2011; Mettke-Hofmann 2014; Ducatez et al. 2015). This provides the generalist with the advantage to flexibly respond to variable environmental conditions, whereas specialists experience advantages over generalists in more stable environments (Lahann et al. 2006; Dewar and Richard 2007; Wilson and Yoshimura 2008). Specifically, specialists may exhibit better long-term memory which allows to use and improve specific routes during habitat exploration, and to discriminate between and relocate former food patches (Mettke-Hofmann 2014).

Despite suggestive covariations between cognitive abilities and the degree of ecological specialization, respective empirical studies have rarely been conducted. Instead, it remains hypothesized that generalist species exhibit better cognitive abilities per se (Parker 1978). Supporting this, generalists are more likely to innovate, and innovative propensity has been reported to correlate with general intelligence and, further, with brain size measures (Reader 2003; Reader et al. 2011; Ducatez et al. 2015). Additionally, self-control is enhanced in dietary generalist primates and dietary generalist birds and primates developed larger brains than dietary specialists (Reader 2003; Lefebvre et al. 2004; Sol et al. 2005b; MacLean et al. 2014; Ducatez et al. 2015).

Yet, for an overall conclusion, we lack systematic investigations across a broader variety of cognitive abilities. Therefore, in the scope of my thesis, I reviewed the literature on comparative approaches that experimentally assessed interspecific variation in cognitive abilities in relation to ecological factors. With this basis of previously reported common links across studies, I empirically investigated interspecific variation across various cognitive abilities with the use of a comprehensive cognitive test battery applied to wild non-human primates. As study species served two sympatric species of mouse lemurs that experience similar environmental conditions and possess comparable social systems, while exhibiting different adaptations to ecological factors, including different degrees of ecological specialization. 


\section{Studying mouse lemurs}

Studying non-human primates is specifically of broad interest, due to their close relatedness to us humans. Researchers hope to shed light on our distinctiveness to nonhuman animals and on the evolutionary success of our own species. In the scope of the present topic to investigate variation in cognitive abilities in relation to ecological factors, mouse lemurs (Microcebus spp.) represent a suitable study organism. These small (appr. $31-100$ g), nocturnal, and primarily solitary lemurs encompass 25 described species that are endemic to Madagascar but occupy different ecological niches (Yoder et al. 2000; Mittermeier et al. 2008; Schüßler et al. 2020). Adaptations to ecological conditions can even differ across populations of one species that are exposed to different environments (Ganzhorn and Schmid 1998; Lahann et al. 2006; Schülke and Ostner 2007; Zimmermann and Radespiel 2014). In several regions, at least two mouse lemur species occur in sympatry, usually with one species being more ecologically specialized than the other (Schmid and Kappeler 1994; Rakotondravony and Radespiel 2009; Rakotondranary and Ganzhorn 2012). In central Western Madagascar, the generalist gray mouse lemur (M. murinus) and the more specialized Madame Berthe's mouse lemur (M. berthae) occur in sympatry in parts of the remaining dry-deciduous lowland forests, as at my study site in Kirindy forest (Schwab and Ganzhorn 2004).

The larger gray mouse lemur $(60 \mathrm{~g})$ is a habitat and dietary generalist, occurring across various habitat types (Lahann et al. 2006; Mittermeier et al. 2010), including highly degraded forest fragments (Ganzhorn and Schmid 1998; Ganzhorn et al. 2012), and experiencing a broad dietary breadth (Dammhahn and Kappeler 2008a). The World's smallest primate, the Madame Berthe's mouse lemur ( $31 \mathrm{~g})$ is a habitat and dietary specialist, occurring only in dry-deciduous lowland forest in central Western Madagascar (Rasoloarison et al. 2000; Schäffler and Kappeler 2014), and experiencing a narrower dietary breadth (Dammhahn and Kappeler 2008a). In Table 1.1, I summarize the main species-specific characteristics concerning socio-ecological factors, as well as prior research efforts in the broad context of cognition and the currently assigned IUCN states. In summary, the two species differ in various ecological adaptations, while social characteristics are largely comparable. As they occur in sympatry at my study site in Kirindy forest, they experience similar environmental characteristics. The fact that they, 
Table 1.1 Main socio-ecological characteristics of gray and Madame Berthe's mouse lemurs, as well as IUCN state and cognitive research effort. Dietary breadth (B) based on Levin's standardized index (Krebs 1989)

\begin{tabular}{|c|c|c|}
\hline & Gray mouse lemur & Mme Berthe's mouse lemur \\
\hline $\begin{array}{l}\text { mean body mass }{ }^{1,2} \\
\text { distribution }^{1,2} \\
\text { occurrence }\end{array}$ & $\begin{array}{l}60 \mathrm{~g} \\
\text { throughout western Madagascar } \\
\text { diverse: evergreen littoral forest, } \\
\text { dry-deciduous forest, primary } \\
\text { and secondary forest, degraded } \\
\text { forest fragments } 3\end{array}$ & $\begin{array}{l}31 \mathrm{~g} \\
\text { central western Madagascar } \\
\text { specific: dry-deciduous } \\
\text { lowland forest, primary } \\
\text { forest only }{ }^{4}\end{array}$ \\
\hline \multicolumn{3}{|l|}{ diet } \\
\hline $\begin{array}{l}\text { dietary breadth } \\
\text { - } \text { composition }^{5}\end{array}$ & $\begin{array}{ll}\text { - } & \text { broad }(B=0.63) \\
\text { - } & \text { patchy homopteran } \\
\text { secretions }(59 \%), \text { abundant } \\
\text { animal matter }(17 \%), \\
\text { stationary fruits/flowers } \\
(9 \%), \text { patchy/stationary gum } \\
(9 \%), \text { others }(6 \%)\end{array}$ & $\begin{array}{ll}\text { - } & \text { narrow }(\mathrm{B}=0.12) \\
\text { - } & \text { patchy homopteran } \\
& \text { secretions }(82 \%), \text { mobile } \\
\text { insects }(11 \%), \text { stationary } \\
\text { fruits/flowers }(2 \%), \\
\text { patchy/stationary gum } \\
(0.2 \%), \text { others }(5 \%)\end{array}$ \\
\hline $\begin{array}{l}\text { ecological } \\
\text { specialization }{ }^{4,6} \\
\text { energy saving } \\
\text { strategies }^{7}\end{array}$ & $\begin{array}{l}\text { habitat generalist } \\
\text { dietary generalist }\end{array}$ & $\begin{array}{l}\text { habitat specialist } \\
\text { dietary specialist }\end{array}$ \\
\hline $\begin{array}{ll}\text { - } & \text { males } \\
\text { - } & \text { females }\end{array}$ & $\begin{array}{ll}\text { - } & \text { short-term torpor } \\
\text { - } & \text { short- \& long-term torpor }\end{array}$ & $\begin{array}{l}\text { - } \text { short-term torpor } \\
\text { - } \text { short-term torpor }\end{array}$ \\
\hline home range size ${ }^{8}$ & & \\
\hline - males & - 2.8 ha & - 4.9 ha \\
\hline - females & - 1.7 ha & - 2.4 ha \\
\hline sleeping sites & mainly tree holes ${ }^{9}$ & mainly leave nests 8 \\
\hline sociality & $\begin{array}{l}\text { solitary forager, formation of } \\
\text { sleeping groups, cooperative } \\
\text { breeder }^{10}\end{array}$ & $\begin{array}{l}\text { solitary forager, solitary } \\
\text { resting, formation of } \\
\text { sleeping groups }\end{array}$ \\
\hline mating season & October - November ${ }^{11}$ & November ${ }^{8}$ \\
\hline cognition research & $\begin{array}{l}\text { diverse: captivity \& wild, social } \\
\& \text { physical abilities, personality, } \\
\text { aging effects }^{12}\end{array}$ & $\begin{array}{l}\text { wild only, innovation, } \\
\text { personality }{ }^{13}\end{array}$ \\
\hline IUCN state & least concern ${ }^{14}$ & critically endangered ${ }^{15}$ \\
\hline
\end{tabular}

${ }^{1}$ Schmid and Kappeler 1994; Rasoloarison et al. 2000; ${ }^{2}$ Mittermeier et al. 2010; ${ }^{3}$ Ganzhorn and Schmid 1998; Lahann et al. 2006; ${ }^{4}$ Schäffler and Kappeler 2014; ${ }^{5}$ Dammhahn and Kappeler 2008a; ${ }^{6}$ Dammhahn and Kappeler 2008b; ${ }^{7}$ Schmid 1999; Schmid et al. 2000; Dammhahn and Kappeler 2012; 8 Dammhahn and Kappeler 2005; ${ }^{~}$ Schmid 1998; ${ }^{10}$ Eberle and Kappeler 2006; ${ }^{11}$ Eberle and Kappeler 2004a; ${ }^{12}$ Schilling 1979 ; Joly et al. 2004; Picq 2007; Dammhahn 2012; Joly et al. 2014b; Huebner et al. 2018; ${ }^{33}$ Henke-von der Malsburg and Fichtel 2018; ${ }^{14}$ Reuter et al. 2020; ${ }^{15}$ Markolf et al. 2020 


\section{Chapter I: General introduction}

nevertheless, occupy different ecological niches, assumes complex evolutionary processes for ecological adaptations that may be linked to cortical development, and may covary with cognition. Hence, they are suitable study species to investigate patterns of covariation of interspecific differences in cognitive abilities and adaptations to ecological factors in the wild.

Assuming, that social factors cannot solely explain variation in cognition (Rosati 2017a), I expected interspecific variation in cognitive performances in relation to the species' adaptations to ecological conditions. Specifically, according to the Opportunistic Intelligence Hypothesis, the generalist gray mouse lemur should consistently outperform the specialist Madame Berthe's mouse lemur. Alternatively, species-specific ecological characteristics, irrespective of the degree of ecological specialization, should covary with specific cognitive performances. For instance, since Madame Berthe's mouse lemurs inhabit larger home ranges and feed on more stationary food items (Dammhahn and Kappeler 2008b), they should exhibit better spatial abilities. Instead, gray mouse lemurs feeding more on fruits and flowers (Dammhahn and Kappeler 2008b), should exhibit better causal understanding.

Finally, by studying Madame Berthe's mouse lemurs' various cognitive abilities, I extend our overall knowledge of cognition in non-human primates. Cognitive abilities of gray mouse lemurs have been studied to a large extend in previous studies, both in captivity and in the wild (Lührs et al. 2009; Dammhahn 2012; Schilling 2012; Joly et al. 2014; Huebner et al. 2018; Kittler et al. 2018; Schmidtke et al. 2018; Henke-von der Malsburg and Fichtel 2018; Fichtel et al. 2020). Using a great variety of experimental tasks, we already have knowledge about cognitive abilities in the social and the physical domain, about personality traits, sensory predispositions, as well as aging effects and links with fitness parameters (Jolly 1964; Schilling 1979; Santos et al. 2005; Bons et al. 2006; Joly et al. 2004; Picq 2007; Siemers et al. 2007; Lührs et al. 2009; Dammhahn and Almeling 2012; Languille et al. 2012; Schilling 2012; Huebner et al. 2018; Kittler et al. 2018; Fichtel et al. 2020). Additionally, both species habituate easily to short-term captivity, they are trap happy and their populations have been monitored for over 25 years in Kirindy forest (Eberle and Kappeler 2002; Kraus et al. 2008; Hämäläinen et al. 2014b; Huebner et al. 2018; Henke-von der Malsburg and Fichtel 2018). Thus, wild populations of mouse lemurs at 
this study site are particularly suited to examine their cognitive skills using a comprehensive test battery that requires many experimental sessions and, accordingly, the chance to repeatedly recapture single individuals.

\section{This study}

The specific aim for my thesis was to broaden our understanding of cognitive evolution in relation to ecological factors. Therefore, I empirically investigated patterns of covariation between cognitive performances and species-specific adaptations to ecological conditions, specifically the degree of ecological specialization.

Specifically, I answered the questions (1) how ecological factors, including the degree of ecological specialization, covary with cognitive performances across various species of the animal kingdom (Chapter II), (2) whether the generalist gray mouse lemur consistently outperforms the specialist Madame Berthe's mouse lemur in cognitive performances (Chapter III), (3) to which extent cognitive abilities can covary with ecological factors (Chapter III) and (4) whether cognitive performances in mouse lemurs correlate to general intelligence ( $g$-factor) which further correlates across species ( $G$-factor; Chapter III). For this, I reviewed the literature by selecting experimental comparative studies that investigated variation in cognition in relation to ecological adaptation. To add experimental data to this theoretic approach, and to test formulated hypotheses concerning the evolution of cognition, I conducted a comprehensive cognitive test battery on wild populations of the sympatric gray and Madame Berthe's mouse lemurs, which differ in their degree of ecological specialization.

In Chapter II of my thesis, I start with a summary of proposed hypotheses aiming to explain the evolution of cognition and provide an overview of covariations with brain size, sociality, and ecology. I continue evaluating the results of my literature survey on experimental comparative studies investigating covariations of cognitive performances with ecological factors in non-human animals. Finally, I estimate general patterns of covariation between cognitive performances and the degree of habitat and dietary specialization, the social group size, as well as the brain size. 
In Chapter III, I first introduce the topic of interspecific variation in cognitive abilities across non-human animals and in relation to socio-ecological factors. Then, I describe the species-specific socio-ecological factors characterizing gray and Madame Berthe's mouse lemurs in more detail. For this experimental approach, I applied a test battery comprising several cognitive tasks to investigate innovative problem-solving abilities, persistence, associative and reversal learning abilities, spatial abilities, inhibitory control, means-end understanding, and goal directedness. I included two additional personality tests to measure the individuals' explorative and neophilic tendencies which might confound cognitive test performances. In addition to the investigation of interspecific variation in cognitive abilities, I estimate the probability of general intelligence in mouse lemurs.

In Chapter IV, I summarize and connect the main aspects of the results reported in Chapter II and III. Additionally, I evaluate the gained knowledge in terms of how it helps to understand cognitive evolution and provide ideas of how this data can be used for future studies extending this exciting field of research. 


\section{Chapter II: Literature review}

\section{Linking ecology and cognition: does ecological specialization predict cognitive test performance?}

Johanna Henke-von der Malsburg ${ }^{1,2,3}$, Peter M. Kappeler ${ }^{1,2}$, Claudia Fichtel ${ }^{1,3}$

${ }^{1}$ Behavioral Ecology and Sociobiology Unit, German Primate Centre, Leibniz Institute for Primatology, Göttingen, Germany

${ }^{2}$ Department of Sociobiology/Anthropology, Johann-Friedrich-Blumenbach Institute of Zoology and Anthropology, Georg-August-University Göttingen, Germany

${ }^{3}$ Leibniz ScienceCampus ‘Primate Cognition', Göttingen, Germany

This manuscript is published in

Behavioral Ecology and Sociobiology (2020) 74:154

DOI 10.1007/s00265-020-02923-z

Author contributions: JHM, PMK and CF conceptualized the review; JHM reviewed the literature, drafted the manuscript and designed the figures.

JHM, PMK and CF wrote the manuscript.

Note: The original publication has been requested for correction. Here, I include the corrected version. 


\section{Abstract}

Variation in cognitive abilities is thought to be linked to variation in brain size, which varies across species with either social factors (Social Intelligence Hypothesis) or ecological challenges (Ecological Intelligence Hypothesis). However, the nature of the ecological processes invoked by the Ecological Intelligence Hypothesis, like adaptations to certain habitat characteristics or dietary requirements, remains relatively poorly known. Here, we review comparative studies that experimentally investigated interspecific variation in cognitive performance in relation to a species' degree of ecological specialization. Overall, the relevant literature was biased towards studies of mammals and birds as well as studies focusing on ecological challenges related to diet. We separated ecological challenges into those related to searching for food, accessing a food item, and memorizing food locations. We found interspecific variation in cognitive performance that can be explained by adaptations to different foraging styles. Species-specific adaptations to certain ecological conditions, like food patch distribution, characteristics of food items or seasonality also broadly predicted variation in cognitive abilities. A species' innovative problem-solving and spatial processing ability, for example, could be explained by its use of specific foraging techniques or search strategies, respectively. Further, habitat generalists tended to outperform habitat specialists. Hence, we found evidence that ecological adaptations and cognitive performance are linked, but that the classification concept of ecological specialization cannot sufficiently explain variation in cognitive performance.

\section{Keywords}

cognition, ecological adaptation, foraging style, habitat complexity, Ecological Intelligence Hypothesis, brain size

\section{Introduction}

Cognition can be defined as the ability to perceive, memorize and process information from an individual's social as well as ecological environment (Shettleworth 2009), and variation in this ability is thought to be positively correlated with brain size. Relative brain 
size varies considerably among species (e.g., Sol et al. 2008; Mace et al. 2009) and is indeed associated with variation in average species-typical cognitive performance (Deaner et al. 2006; Reader et al. 2011). Several hypotheses have been proposed to explain this link between interspecific variation in brain size and the associated cognitive abilities. The most prominent hypotheses are the Social Intelligence Hypothesis (SIH; Humphrey 1976) and the Ecological Intelligence Hypothesis (EIH; Parker and Gibson 1977; Milton 1988), which have been subsequently refined as the Domain-General Hypothesis (Deaner et al. 2006), Domain-Specific Hypothesis (Whiten and Byrne 1988), Adaptive Intelligence Hypothesis (Tooby and Cosmides 2003), Machiavellian Intelligence Hypothesis (Whiten and Byrne 1988), Cultural Intelligence Hypothesis (Herrmann et al. 2007; van Schaik and Burkart 2011), Social Brain Hypothesis (Dunbar 1998; Dunbar et al. 2007) and Cognitive Buffer Hypothesis (CBH; Deaner et al. 2003) (see Fig. 2.1) .

The SIH suggests that bigger brains co-evolved with increasing social complexity, i.e., cognitive challenges to manage social relationships, which, in turn, have evolved as means of solving ecological problems (Jolly 1966; Humphrey 1976; Whiten and Byrne

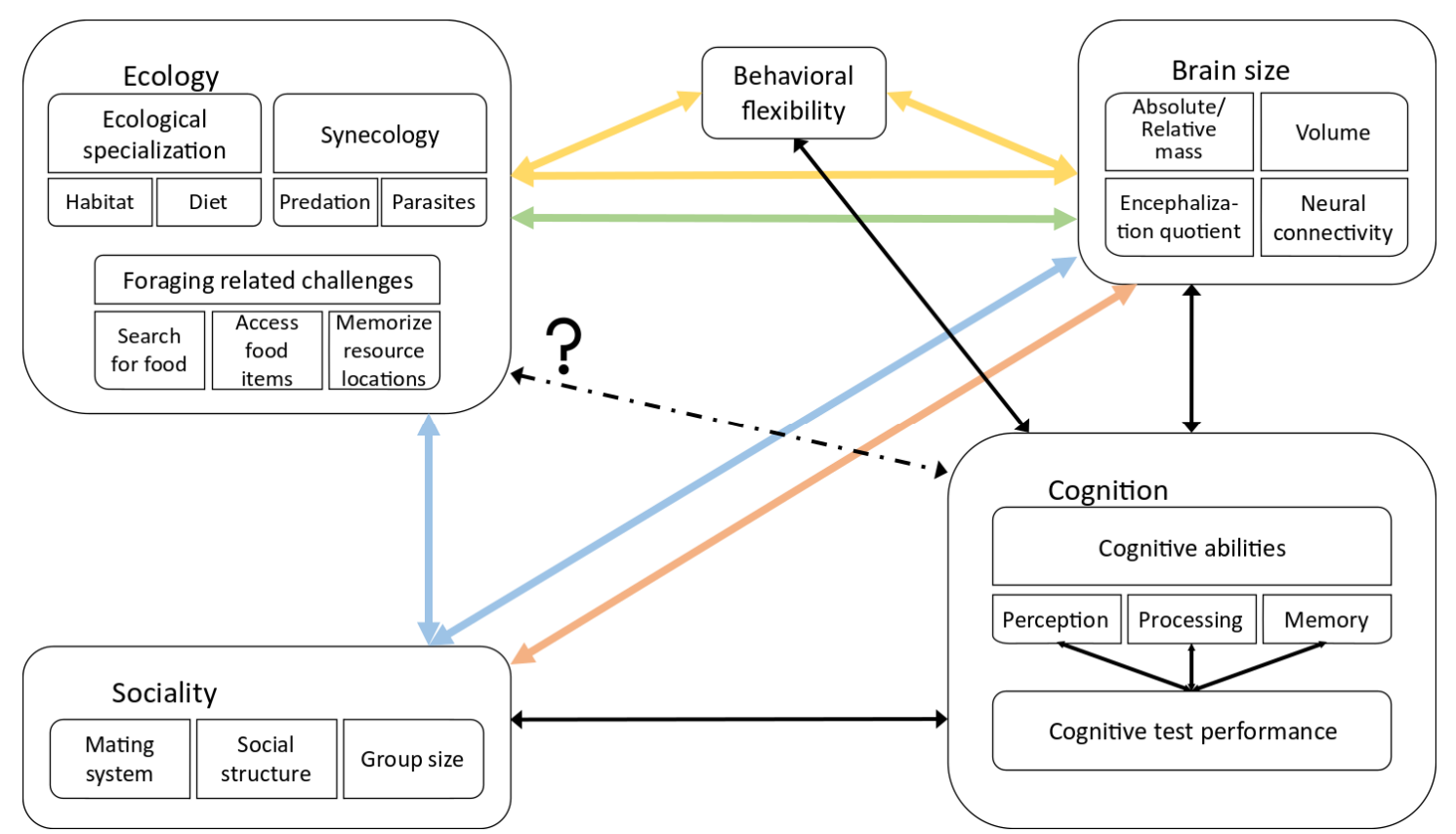

Fig. 2.1 Relationships between ecology, sociality, brain size measures and cognitive abilities. Main hypotheses related to particular links are represented with colored thick lines: red for the Social Intelligence Hypothesis (challenges related to sociality drive brain development; Humphrey 1976), green for the Ecological Intelligence Hypothesis (challenges related to ecology drive brain evolution; Parker and Gibson 1977), blue for the Social Brain Hypothesis (ecological challenges predict sociality which drives brain size; Dunbar 1998; Dunbar and Shultz 2017), and yellow for the Cognitive Buffer Hypothesis (environmental variation drives brain size which favours adaptive behavioural flexibility; Deaner et al. 2003). Thinner black lines represent previously reported relationships between cognitive abilities and sociality, behavioral flexibility, and brain size. The dashed line represents the relationship between cognitive abilities and ecology discussed in this paper 
1988; Barton 1996; Dunbar 1998; Kudo and Dunbar 2001; Dunbar et al. 2007; Byrne and Bates 2010). In contrast, the EIH posits that ecological challenges in food acquisition, including spatial or spatio-temporal processes to memorize seasonally available food or manipulative skills for extractive foraging, have ultimately selected for larger brains (e.g., Clutton-Brock and Harvey 1980; Heldstab et al. 2016; DeCasien et al. 2017; Powell et al. 2017). At the end of the day, brains evolve within species, where environmentally induced changes in physiological traits, such as glucose concentrations and hormone levels, impact cognitive performance (Roth et al. 2010; Thornton and Lukas 2012; Maille and Schradin 2016a).

Comparative studies investigating the link between measures of brain size and socio-ecological factors revealed inconsistent results, finding either a positive relationship between measures of brain size and sociality in primates and ungulates (Dunbar 1998; Pérez-Barbería et al. 2007; Dunbar and Bever 2010) or equivocal support in carnivores (Holekamp et al. 2007; Pérez-Barbería et al. 2007; Finarelli and Flynn 2009), for instance. Among primates, the most recent comparative analyses indicated that brain size is better predicted by ecological than social factors (DeCasien et al. 2017; Powell et al. 2017). However, compared to social factors (Ashton et al. 2018b), less is known about the extent to which variation in specific ecological factors predicts cognitive performance (Fig. 2.1). Here, we therefore explore the question whether ecological adaptations can explain interspecific variation in cognitive abilities by first briefly summarizing hypotheses about the evolution of cognitive abilities addressing potential links between cognition and brain size, sociality, as well as ecology, respectively. Second, we provide a summary of comparative studies relating interspecific variation in cognitive performance with ecology, specifically the degree of ecological specialization. Using these studies, we also explore the relative impact of sociality and brain size on variation in cognitive performance. Finally, we discuss the effects of the degree of ecological specialization, potentially confounding factors in experimental studies, and limitations of this review that may inform future research on this topic. 


\subsection{The link between cognition and brain size}

Some comparative studies have suggested that measures of brain size co-vary positively with performance in cognitive tests (see Appendix 1, Table S1-1 for explanations of cognitive terms), such as performance in inhibitory control across vertebrates (MacLean et al. 2012), a general intelligence ( $g$ )-factor extracted from performance in innovation, social learning, tool use, extractive foraging and tactical deception (Reader and Laland 2002; Reader et al. 2011), a g-factor extracted from performance in tests on spatial and causal understanding, reversal learning and delayed response (Deaner et al. 2006, 2007), or performance in problem-solving (Benson-Amram et al. 2016). In addition, guppies (Poecilia reticulata) selected for larger brains outperformed those with smaller brains in a numerical learning assay (Kotrschal et al. 2013), demonstrating this link also at the intraspecific level.

However, it is questionable whether simple measures of brain size can be used to infer its impact on the solution of a specific problem (Healy and Rowe 2007). In addition, different neuroanatomical measures of brain size have been combined with inconsistently labelled methods and various cognitive response measures (Healy and Rowe 2007). To circumvent these problems, some studies extracted a general intelligence factor, which is a statistical value derived from correlating performance in several cognitive tasks (Spearman 1904; Jensen 1985). In primates (Reader and Laland 2002; Deaner et al. 2006; Deaner et al. 2007; Reader et al. 2011), the g-factor co-varied positively with brain size. Although some other studies provided evidence for a g-factor in humans (Jensen 1985), non-human primates (Fernandes et al. 2014; Damerius et al. 2019), rodents, rabbits, cats, dogs (Galsworthy et al. 2014), and birds (Sol et al. 2005a; Ducatez et al. 2015), we lack validation that this correlation factor represents general intelligence (Burkart et al. 2017). In fact, it can simply reflect the selection of cognitive tasks that tap into similar cognitive domains, which would not necessarily validate the notion of general intelligence (Shaw and Schmelz 2017; Bräuer et al. 2020).

Alternatively, domain-specific cognition might support the notion of mosaic brain evolution (Barton and Harvey 2000). The vertebrate brain consists of several functionally different structures, of which many vary in size within and between clades (Striedter 2006). Differences in the relative size of different brain regions are thought to reflect both 
neurodevelopmental/functional size changes and selection for ecologically relevant cognitive and sensory specialization (Barton 1996). For example, food-caching birds have relatively, but not absolutely larger hippocampi (Krebs 1990), and brain regions associated with spatial processing are enlarged in species with better spatial abilities (Sherry et al. 1992; Clayton 1998). Moreover, group-living primates with high-quality diets have larger brain regions for olfactory or visual processing, whereas solitary species or those with lowquality diets have larger brain regions for processing spatial memory (DeCasien and Higham 2019). Finally, the number of neurons in the mammalian cerebral cortex, or in the bird pallium, appear to be good predictors of inhibitory control (Herculano-Houzel 2017). Hence, specific neuroanatomical measures can be broadly associated with variation in cognitive performance.

\subsection{The link between cognition and sociality}

Cognitive abilities have been linked to traits associated with variation in social factors. Variation in sociality is often operationalized in terms of group size (Lukas and CluttonBrock 2013; Kappeler 2019), which varies from solitary individuals and small pair-bonded units to large aggregations. The need for several social skills, like effective communication or coordination with other group members, requires specific neural structures (Dunbar and Shultz 2007; Peckre et al. 2019). Thus, group size or group dynamics are contributing to the complex social challenges an animal faces (Kappeler 2019).

Accordingly, primates living in dynamic fission-fusion systems (Aureli et al. 2008) performed better in inhibitory control tasks than those living in more stable groups, suggesting that the former exhibit greater behavioral flexibility (Amici et al. 2008; Amici et al. 2018). In lemurs and birds, species organized into more complex social groups outperformed others in transitive inference tasks (Bond et al. 2003; MacLean et al. 2008). A similar inter-specific difference was found in birds subjected to a reversal-learning task (Bond et al. 2007). Moreover, within species, Australian magpies (Gymnorhina tibicen dorsalis) living in larger groups performed better in several cognitive tests (inhibitory control, associative and reversal learning abilities, spatial memory and innovation) than those living in smaller groups (Ashton et al. 2018a, 2019). Since traits such as inhibitory 
control and (reversal) learning abilities reflect behavioral flexibility, the underlying variation in cognition can be related to variation in sociality.

\subsection{The link between cognition and ecology}

Ecological challenges, such as variable schedules of resource availability, habitat complexity, predation risk or parasite exposure, may have contributed to cognitive evolution (Garamszegi et al. 2007; Lefebvre and Sol 2008; Shumway 2008; Soler et al. 2012; Morand-Ferron et al. 2016; Sayol et al. 2016). With respect to resource variation, the CBH posits that larger brains evolved to allow species to adjust their behavior adaptively in response to variable environmental conditions (Deaner et al. 2003). For instance, frugivorous species, which have on average larger brains than folivorous species, rely more on resources that vary in their spatiotemporal distribution than folivorous species (Clutton-Brock and Harvey 1980; Mace et al. 2009). Moreover, birds and Old World primates exposed to seasonal changes in food availability tend to have larger brains than species living in non-seasonal habitats (van Woerden et al. 2012; Sayol et al. 2016). Innovativeness, which is adaptive in variable environmental conditions, correlates positively with brain size in primates and birds (Reader and Laland 2002; Lefebvre et al. 2004; Overington et al. 2009), but it also correlates positively with parasitism and immunocompetence in birds (Møller et al. 2005; Garamszegi et al. 2007; Vas et al. 2011), though the link between innovation and the transmission mode of parasite types is not obvious (Ducatez et al. 2020a). Finally, in line with the EIH, brain size was best predicted by diet or home range size in primates (DeCasien et al. 2017; Powell et al. 2017).

Comparisons within species also revealed evidence that variation in cognitive abilities is associated with parasitism (Dunn et al. 2011; Bókony et al. 2014), predation (Brown and Braithwaite 2005; Park et al. 2008; Ferrari 2014), habitat complexity (Roth et al. 2010; Tebbich and Teschke 2014; Croston et al. 2017; Morand-Ferron et al. 2019), or foraging behavior (Mazza et al. 2019; Sonnenberg et al. 2019). However, only a few comparative studies explicitly explored which cognitive skills might be associated with which species-specific ecological challenges. In birds, innovation was positively correlated with parasitism (Garamszegi et al. 2007; Vas et al. 2011; Soler et al. 2012) or habitat breadth 
(Overington et al. 2011), but not with predation (Overington et al. 2011). In primates, abundance of socially transmitted parasites were positively associated with rates of social learning, and environmentally transmitted parasites were positively associated with rates of exploration (McCabe et al. 2015). Habitat complexity was positively correlated with spatial cognition and brain size in rodents (Mackay and Pillay 2017) and fish (White and Brown 2015a). Hence, some results point towards an association between cognitive performance and ecological adaptations and a systematic summary of respective associations across species may contribute to a better understanding of potential causalities and their relative effect sizes.

The required assessment of a species' ecological adaptation can be based on a characterization of its niche, which is a multidimensional space comprising all ecological factors that determine species viability (Hutchinson 1953; MacArthur 1957). Specialization and generalization represent the extremes of the continuous variation along each of the dimensions (Sargeant 2007). Utilizing this niche concept, however, involves some difficulties. First, it is important to differentiate between intrinsic specializations due to evolutionary adaptations (i.e., an individual's genetics describing its fundamental niche) and extrinsic specializations due to interspecific competition over resources (i.e., the observed realized niche), for instance (Hutchinson 1957; Devictor et al. 2010). Second, niche breadth can be defined by the diversity of resources used by a species, or by its overlap, measured as the deviation from other species' resource values (Sargeant 2007). Considering niche breadth, a specialist would then be a species consistently using a narrower niche than other species (Roughgarden 1972; Bolnick et al. 2003). Considering niche overlap, however, a specialist would use items/tactics that are rarely used within other species' niches (Bolnick et al. 2002). Moreover, classifications refer to only one niche axis (Futuyma and Moreno 1988), leading to species that can be highly specialized along one ecological gradient while being a generalist along another ecological gradient. It has therefore been proposed to use species co-occurrence as a measure of habitat breadth (Ducatez et al. 2014b). Finally, different studies use different terms for similar concepts without stating their definition, or they ignore differences between niche breadth and niche overlap, hampering broader comparisons (Colwell and Futuyma 1971; Devictor et al. 2010). 
We, thus, use the concept of ecological specialization as formalized by Hutchinson (Hutchinson 1957) and similarly to Hughes (Hughes 2000). Accordingly, we consider a dietary specialist as a species consuming a lower variety of food types and a habitat specialist as a species occurring in a lower variety of habitat types than a dietary generalist or habitat generalist, respectively. We use this distinction always relative to the species in comparison, not as an absolute attribute. Since animals are expected to have evolved cognitive adaptations to exploit these respective conditions as efficiently as possible (Mettke-Hofmann 2014), generalists and specialists are expected to vary in their performances across cognitive tasks, but also in some personality traits. Hence, generalists have been suggested to be more explorative, to have better working memory, to learn faster, to exhibit greater behavioral flexibility and to have a higher innovative potential than specialists. Specialists are instead expected to exhibit better long-term memory, despite smaller brains, than generalists (Reader 2003; Mettke-Hofmann 2014).

Below, we review relevant studies that explicitly investigated inter-specific variation in cognitive performance posed by problems that are related to species-specific adaptations to factors reflecting the degree of ecological specialization. Using the search query "(ecolog* or generali* or speciali* or "lifestyle" or opportunist*) and (cogniti* or learn* or memory) and animal" in "topic" (including titles, abstracts, keywords, and keywords plus) in the Web of Science [https://apps.webofknowledge.com/, accessed on 2019-07-14] and complementing the collection with other relevant studies via crossreferences, we found a total of $\mathrm{N}=25$ studies, that fit our criteria.

All selected studies (1) experimentally compared (2) cognitive abilities between (3) at least two species with the aim to investigate (4) different ecological adaptations with a focus on the degree of ecological specialization. We controlled for potential phylogenetic effects by excluding studies comparing species across taxonomic classes. The remaining studies either compared species within the same genus (40\%), family (48\%), or order (12\%). Most of the studies compared wild-caught or semi-free ranging (72\%) individuals in mammals $(56 \%)$ or birds $(24 \%)(\mathrm{N}=2$ for each in reptiles and fish, $\mathrm{N}=1$ for invertebrates). The investigated ecological adaptations were mostly related to dietary challenges and variation in habitat complexity (Table 2.1). As studies of cognitive performance often differ in experimental conditions (animal housing, feeding regimes, 


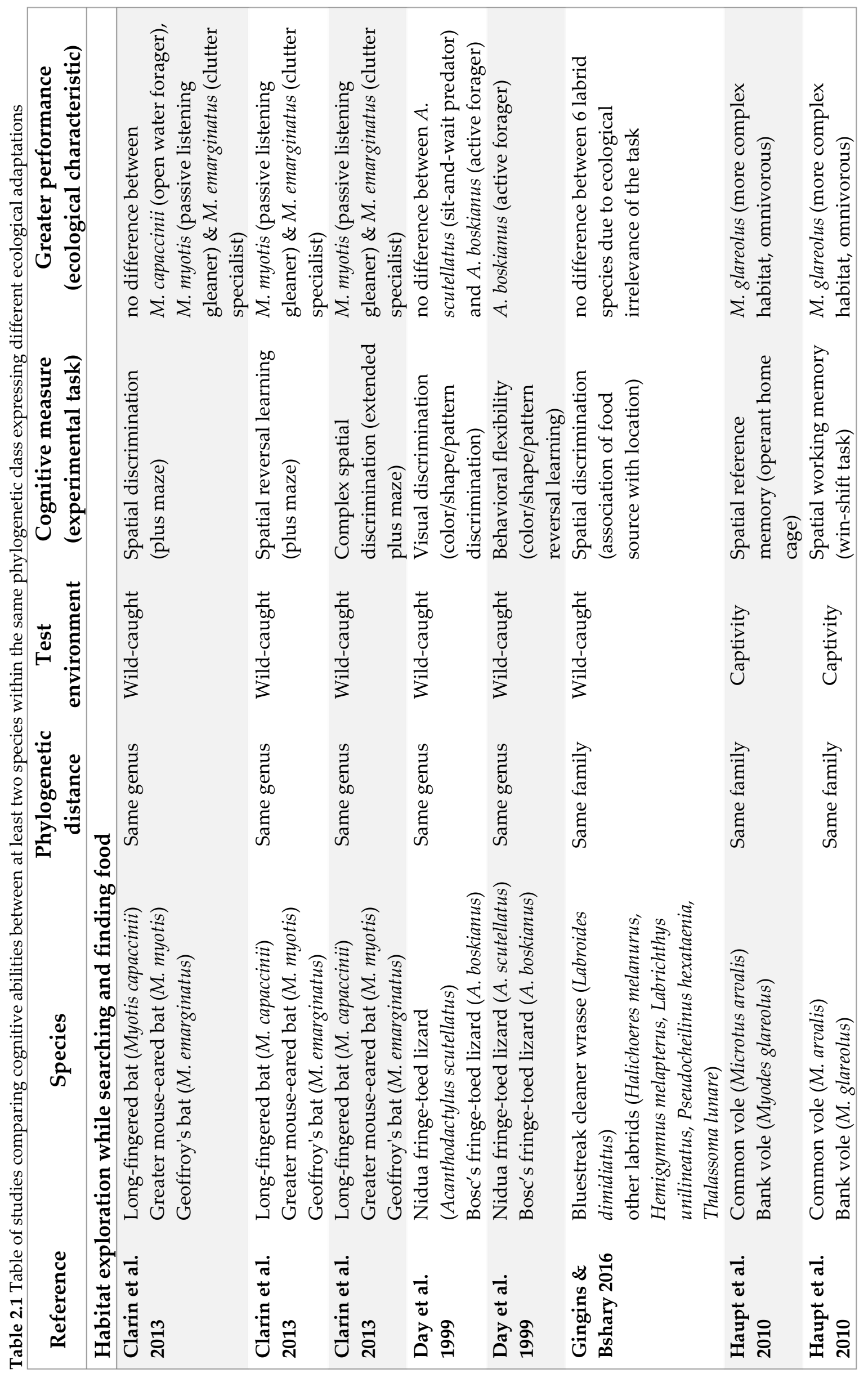




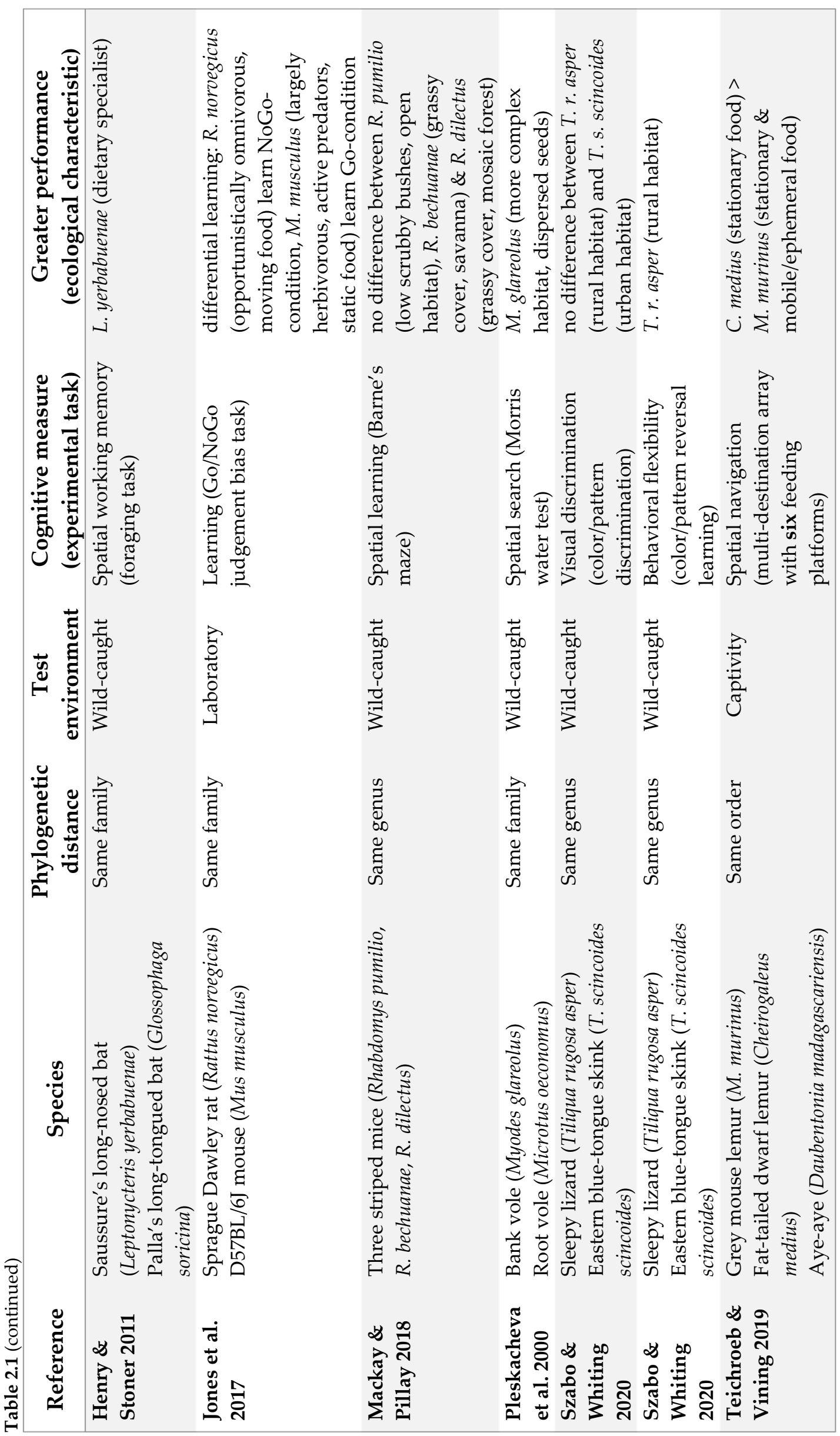



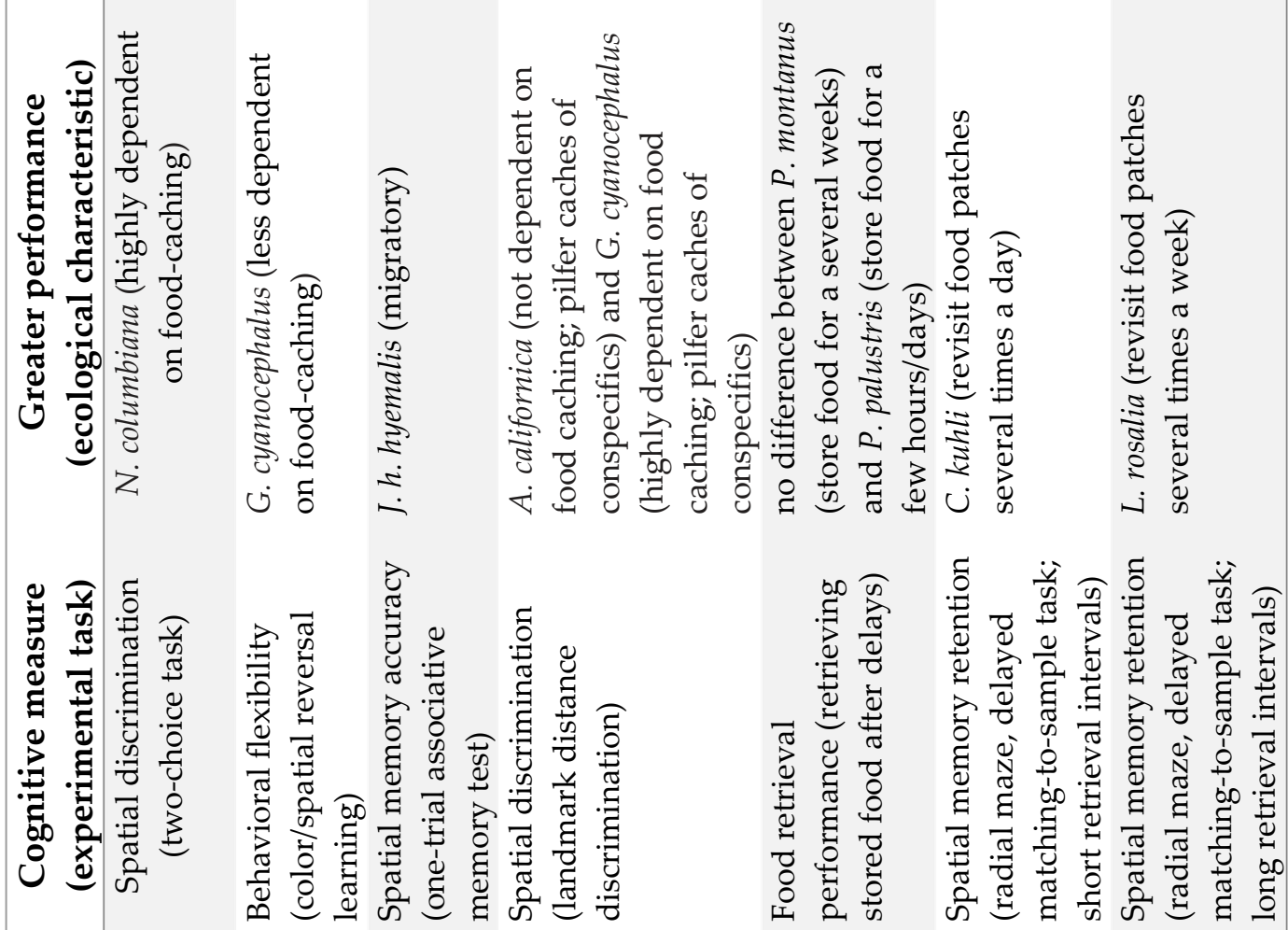

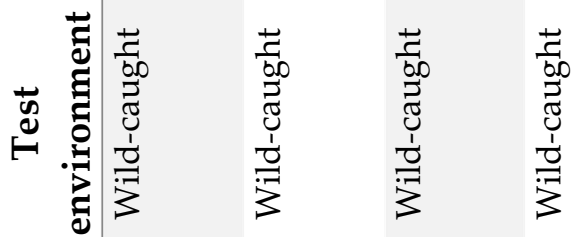
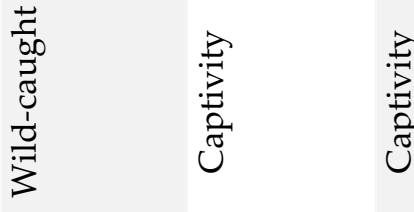

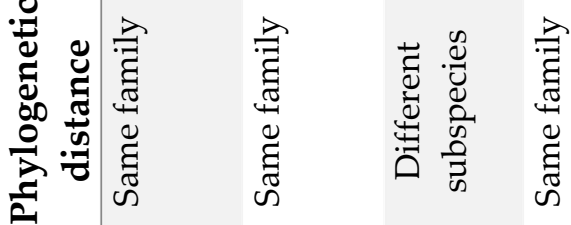

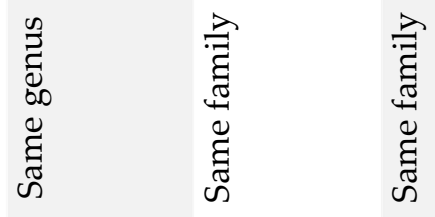

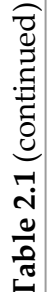
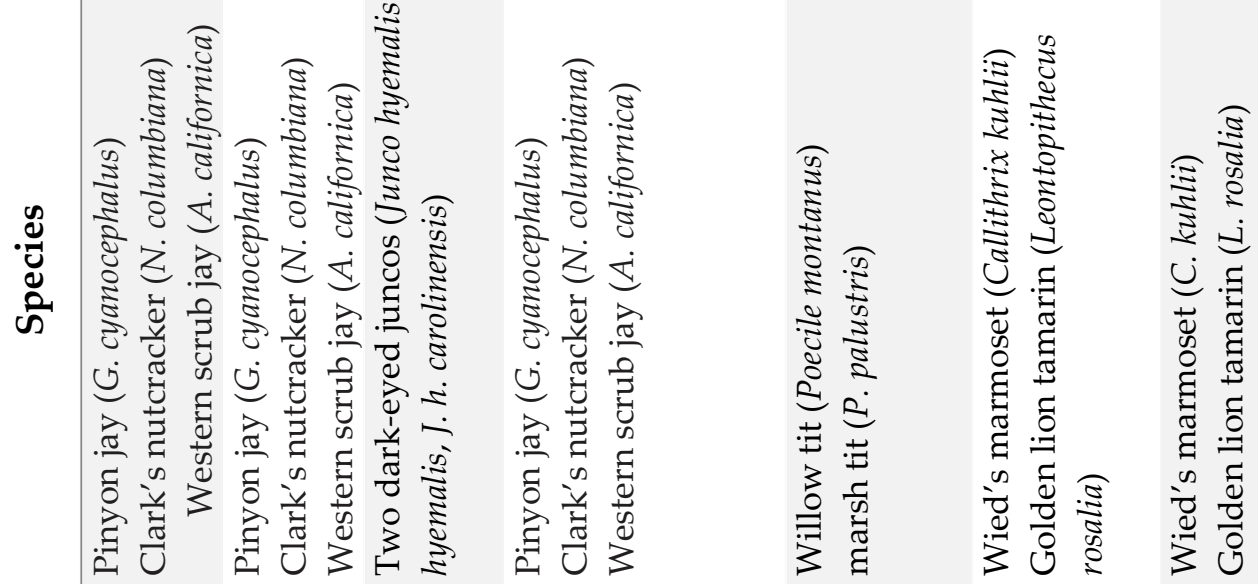

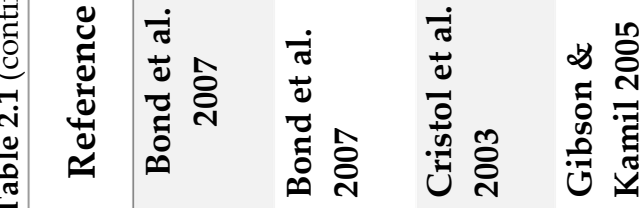

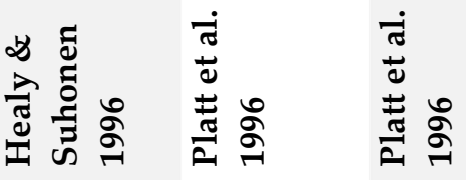




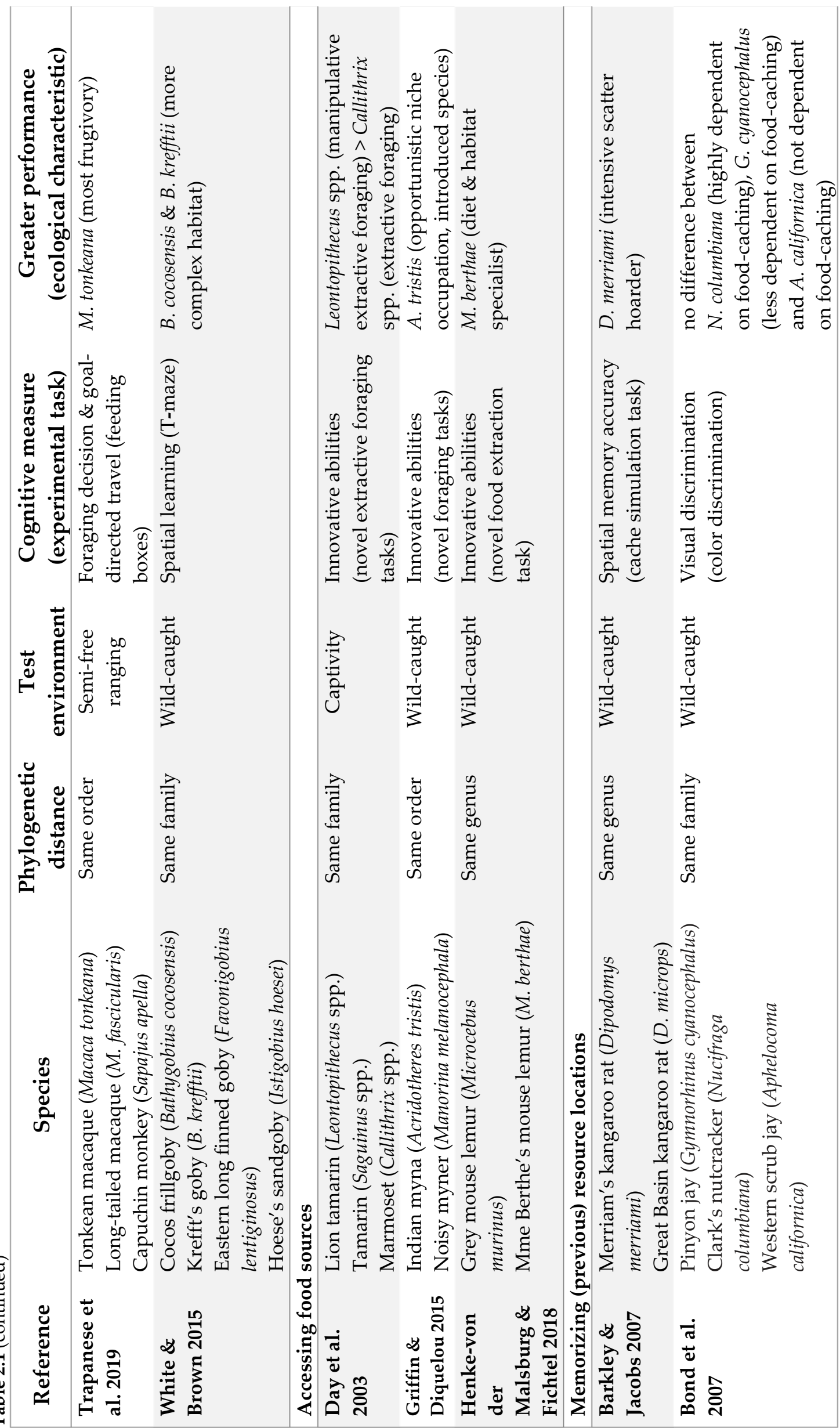


Chapter II: Literature review

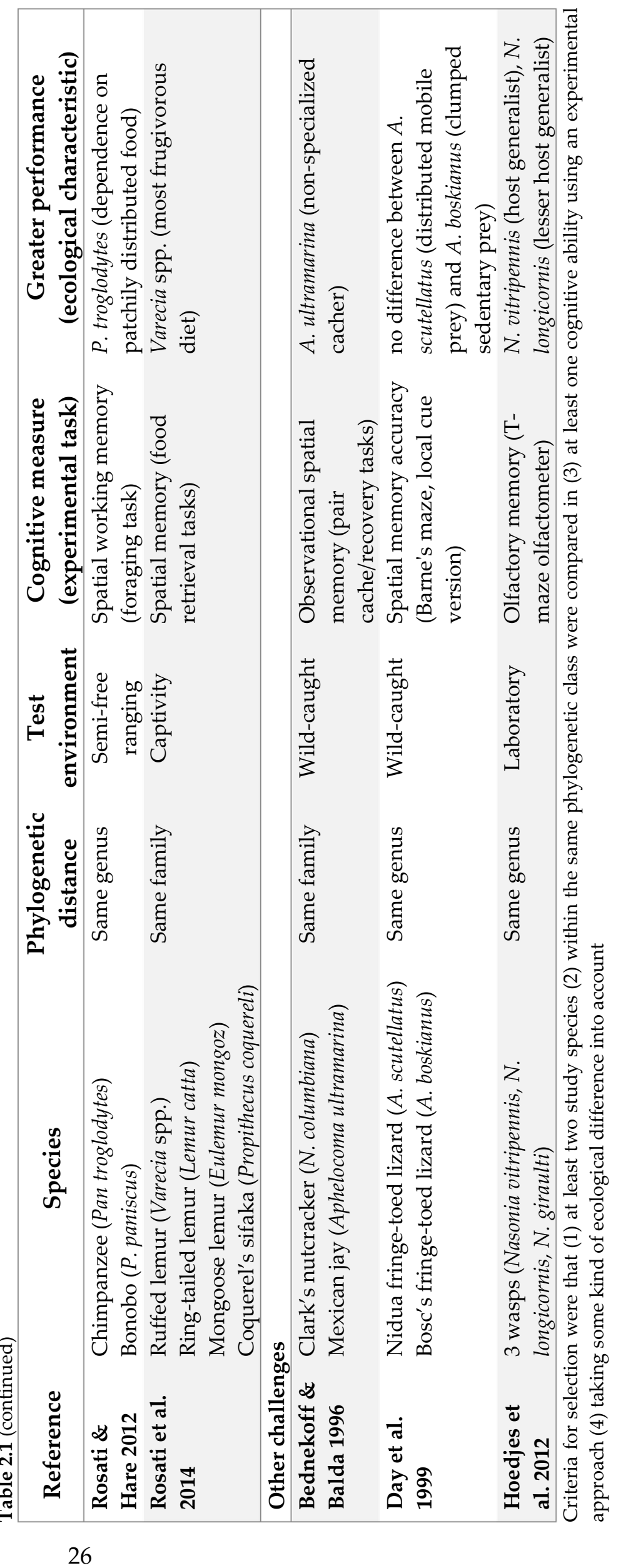


environmental conditions, local and temporal conditions, experimental task and procedure, role of experimenter, etc.), measurement of cognitive performance and analysis in interspecific comparisons, we focused on explicitly comparative studies. Since only a few species were explicitly labelled as either generalist or specialist in the original studies, we assessed the relative degrees of habitat and/or dietary specialization between the species investigated using additional literature. Similarly, if not mentioned in the original study, we gleaned information on group and brain size or proxies for brain size by consulting additional literature (Table 2.2).

\section{Cognitive performance and ecological specialization}

Ecological challenges are of various nature, including the avoidance of predators and parasites and securing access to shelter or resources. However, most existing studies investigated variation in cognitive performance related to experienced foraging challenges. In the following, we distinguish among challenges related to habitat exploration while searching and finding food, accessing food items, and memorizing previous resource locations, and summarize their associations with the degree of ecological specialization.

\subsection{Habitat exploration while searching and finding food}

Exploring different habitat types while searching for food may require different foraging techniques. More complex habitats with variable and unpredictable environments may require superior spatial learning abilities, allowing animals to flexibly adjust to these variable environments. Bats have been widely studied across habitats since their echolocation varies with landscape features (Schnitzler et al. 2003). Geoffroy's bats (Myotis emarginatus) and greater mouse-eared bats (M. myotis), which forage in more complex and less stable habitats, learned a complex spatial discrimination faster and showed more flexibility when reward contingencies changed, than long-fingered bats (M. capaccinii), which forage in simpler and more stable open water habitats (Clarin et al. 2013). Similarly, Cocos frillgobies (Bathygobius cocosensis) and Krefft's gobies (B. krefftii) that occur in 


\section{Chapter II: Literature review}

spatially complex rock pool habitats performed better in a spatial learning test than Eastern long finned gobies (Favonigobius lentiginosus) and Hoese's sandgobies (Istigobius hoesei) occurring in homogenous sandy shores (White and Brown 2015). Also bank voles (Myodes glareolus), which occur in more complex habitats, exhibited better spatial search behavior than root voles (Microtus oeconomus), which occur in more open habitats (Pleskacheva et al. 2000). Moreover, omnivorous bank voles and herbivorous common voles (M. arvalis) were similar in their efficiency of exploiting stable habitats, but the omnivorous bank voles were more efficient in exploiting habitats with temporally changing food locations (Haupt et al. 2019). However, striped mice (Rhabdomy pumillo, $R$. bechuanae, $R$. dilectus) occurring in habitats of different complexity did not differ in spatial learning abilities, possibly due to phylogenetic constraints (Mackay and Pillay 2017).

Finally, adapting to and persisting in urban landscapes have been suggested to be associated with behavioral flexibility in several species (Sih 2013; Sol et al. 2013; Ducatez et al. 2020b). However, Eastern blue-tongue skinks (Tiliqua scincoides scincoides) originating from urbanized areas did not perform better in a reversal learning task than sleepy lizards (T. rugosa asper) originating from rural areas (Szabo and Whiting 2020). Hence, variation in habitat complexity seems to covary with learning abilities in most cases, in particular spatial learning abilities and decision-making.

Regarding the search for food, animals use specific ranging behavior to track the spatio-temporal distribution of dietary items. By using specific navigational heuristics, i.e., decision rules applied to certain situations (Gigerenzer 2008), animals can improve their exploitation of the environment while reducing cognitive effort and energy expenditure. The use of such heuristics varies with the distribution of food items or the mobility of preferred food items. In primates, the more frugivorous Tonkean macaques (Macaca tonkeana) exhibited more goal directed search strategies while foraging than the less frugivorous long-tailed macaques (M. fascicularis) or capuchin monkeys (Sapajus apella; Trapanese et al. 2019). The mobility of food items also influenced search strategies in three lemur species (Teichroeb and Vining 2019). Fat-tailed dwarf lemurs (Cheirogaleus medius), which are specialized on stationary fruits, performed best in a multi-destination array with several feeding platforms. Their superiority was explained by efficient use of specific heuristics for exploration and feeder exploitation. In comparison, dietary generalist grey 
mouse lemurs (Microcebus murinus), which feed on stationary food items like flowers and gum but also on mobile food items such as insects, used fewer heuristics. Aye-ayes (Daubentonia madagascariensis), which are specialized on mobile and ephemeral insect larvae, used basically no heuristics at all, resulting in greater explorative effort and lower cognitive task performance. Similarly, Saussure's long-nosed bats (Leptonycteris yerbabuenae), which are specialized on nectar, foraged more efficiently in a feeder set-up with artificial flowers by revisiting the feeders less often and depleting them more than long-tongued bats (Glossophaga soricina), who complement their nectar and pollen diet additionally with fruits and insects according to seasonal availability (Henry and Stoner 2011). Thus, the reliance on more mobile food items seems to increase exploration, which results in lower task performance in spatial cognition tests.

Variation in cognitive performance is also associated with foraging style. In rodents, faster exploring mice (Mus musculus) learned contingencies between an auditory cue and a food reward or punishment differently than slower exploring rats (Rattus norvegicus). Both rodents could learn either of the reward contingency, i.e., to stay in the initial compartment or move to a second compartment, to receive a food reward. However, they were unable to overcome their baseline activity tendencies to avoid a punishment: the more active mice only reached the learning criterion when they had to move to the second compartment, while rats only reached the learning criterion when they had to stay in the initial compartment to avoid punishment (Jones et al. 2017). In reptiles, the actively foraging Bosc's fringe-toed lizard (Acanthodactylus boskianus) learned a visual reversal learning paradigm faster than the Nidua fringe-toed lizard (A. scutellatus), which is a sit-and-wait predator (Day et al. 1999a). Thus, a more proactive foraging style is consistently associated with superior performance in various cognitive tests.

\subsection{Accessing food items}

Once an animal has solved the problem of locating a given food patch, it encounters the next challenge: the extraction of the actual food item. Depending on the item's characteristics, superior sensorimotor coordination or manipulative skills, including extractive foraging techniques or tool use, are beneficial but also energetically more costly, 
and ultimately require a larger brain (Parker and Gibson 1977; Heldstab et al. 2016). Sensorimotor control and extractive foraging abilities seem to be especially helpful for solving innovative problems, and the majority of innovative behaviors has indeed been recorded in the foraging domain (Reader and Laland 2002). Indian mynas (Acridotheres tristis) which exhibited greater diversity in motor behaviors, were more innovative than noisy miners (Manorina melanocephala) (Griffin and Diquelou 2015). Madame Berthe's mouse lemurs (Microcebus berthae) that expressed better motor control than grey mouse lemurs, subsequently learned a modified innovative problem faster (Henke-von der Malsburg and Fichtel 2018). In seven species of callitrichid monkeys, the existence of extractive foraging techniques predicted innovative abilities, with lion tamarins (Leontopithecus spp.), which are manipulative extractive foragers with a higher innovative potential, exceeding the gum-specialized, extractive foraging marmosets (Callithrix spp.) and the non-extractive, but only visually foraging tamarins (Saguinus spp.) (Day et al. 2003). These examples suggests that efficient access to food sources can vary with cognitive abilities such as innovative behaviors, which also seemed to be influenced by motor coordination.

\subsection{Memorizing previous resource locations}

When animals are highly reliant on cached food or when they migrate to other habitats, usually due to seasonal food shortage (Wall and Stephen 1990; Dingle 2014), they require well developed spatial cognitive abilities. In both cases, animals with good spatial processing abilities are better at memorizing locations of food patches than animals with no or only basic spatial processing abilities (Shettleworth 1990; Sherry et al. 1992). For example, compared to non-migratory dark-eyed juncos (Junco hyemalis carolinensis), migratory dark-eyed juncos (J.h. hyemalis) showed greater accuracy in remembering feeder locations that have been visited only once before a certain retrieval interval (Cristol et al. 2003). Moreover, Merriam's kangaroo rats (Dipodomys merriami), intensive scatter hoarders, showed better spatial memory accuracy in a cache simulation task than Great Basin kangaroo rats (D. microps), leaf-eating specialists that do not rely on scatter-hoarding (Barkley and Jacobs 2007). Furthermore, food caching Clark's nutcrackers (Nucifraga co- 
lumbiana) performed better in an initial spatial discrimination task than less food caching pinyon (Gymnorhinus cyanocephalus) and non-food caching Western scrub jays (Aphelocoma californica). Pinyon jays, however, performed better than the other two corvid species in serial reversal learning tasks (Bond et al. 2007) and Western scrub jays performed better in a distance discrimination task (Gibson and Kamil 2005). Thus, the reliance on food caching can co-vary with spatial memory, but not necessarily with other cognitive abilities.

Although primates do not rely on food-caching, spatial memory abilities can be beneficial for relocating a certain food patch. Indeed, chimpanzees (Pan troglodytes) that feed on more patchy distributed foods than bonobos (P. paniscus) exhibited better retrieval performance when food items were hidden by a human demonstrator (Rosati and Hare 2012). Similarly, lemurs varied in performance in spatial memory tasks according to their diet. The most frugivorous ruffed lemurs (Varecia spp.) showed better spatial memory than ring-tailed lemurs (Lemur catta), mongoose lemurs (Eulemur mongoz) and Coquerel's sifakas (Propithecus coquereli); the latter being the most folivorous species (Rosati et al. 2014). Wied's marmosets (Callithrix kuhlii) performed better than golden lion tamarins (Leontopithecus rosalia) in spatial memory experiments with relatively short retention intervals ( 5 or $30 \mathrm{~min}$ ). However, they performed poorly after longer retention intervals (24 or $48 \mathrm{~h}$ ), while the tamarins could maintain their performance level (Platt et al. 1996). Again, the observed interspecific differences match the species' foraging strategies: while marmosets revisit single food patches several times per day (Rylands 1989), tamarins do so only every three days (Peres 1989). Hence, spatial memory abilities also vary in accordance with a species-typical foraging ecology.

\section{Ecological generalism versus ecological specialization}

Empirical comparisons of cognitive performance between generalists and specialists have either investigated effective habitat exploration in, for instance, spatial memory tasks (Pleskacheva et al. 2000; Haupt et al. 2010), or specific foraging abilities like innovative propensities (Day et al. 2003; Overington et al. 2009; Overington et al. 2011; Griffin and Diquelou 2015; Henke-von der Malsburg and Fichtel 2018), associative learning (Hoedjes 
Chapter II: Literature review

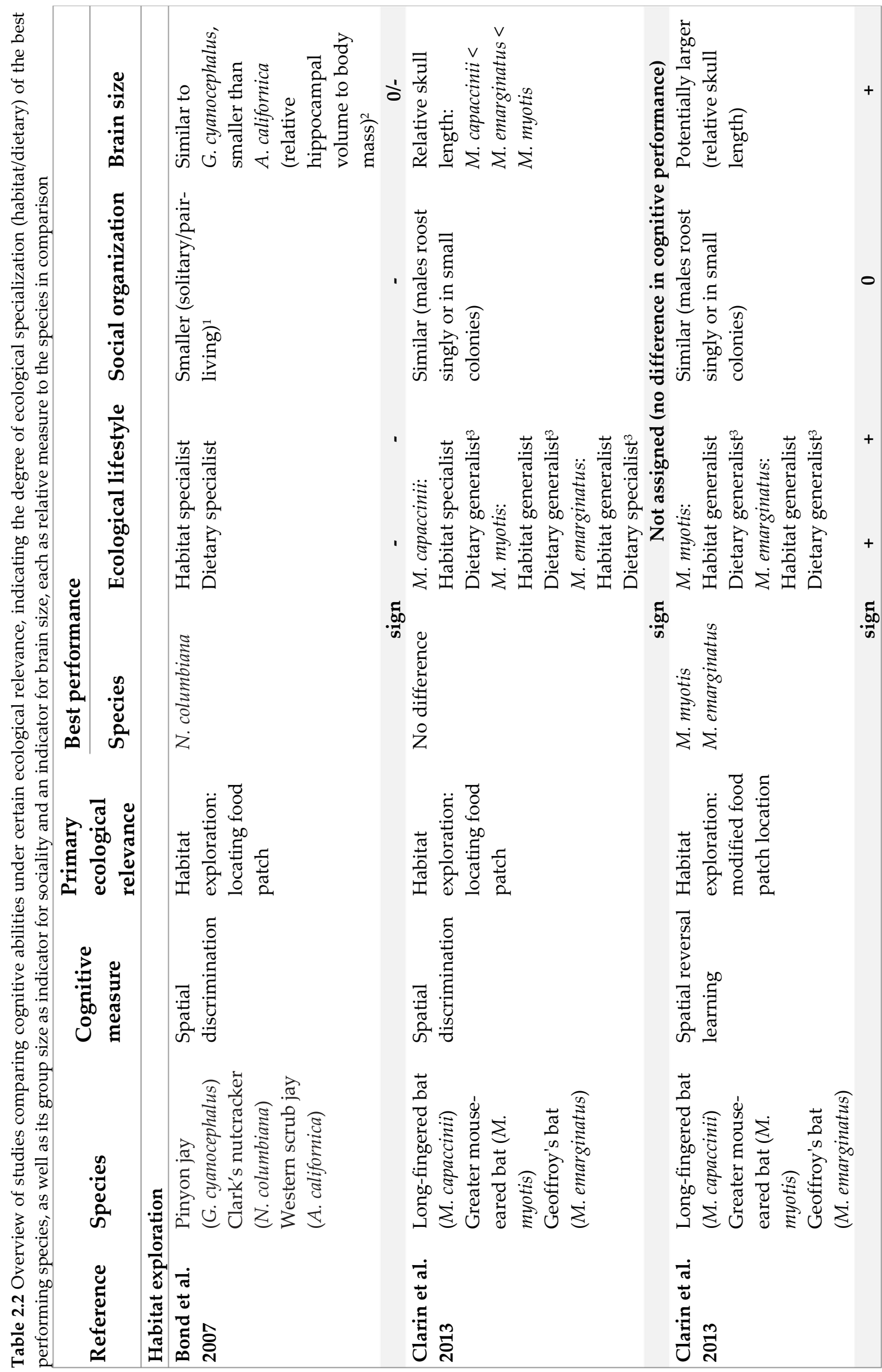




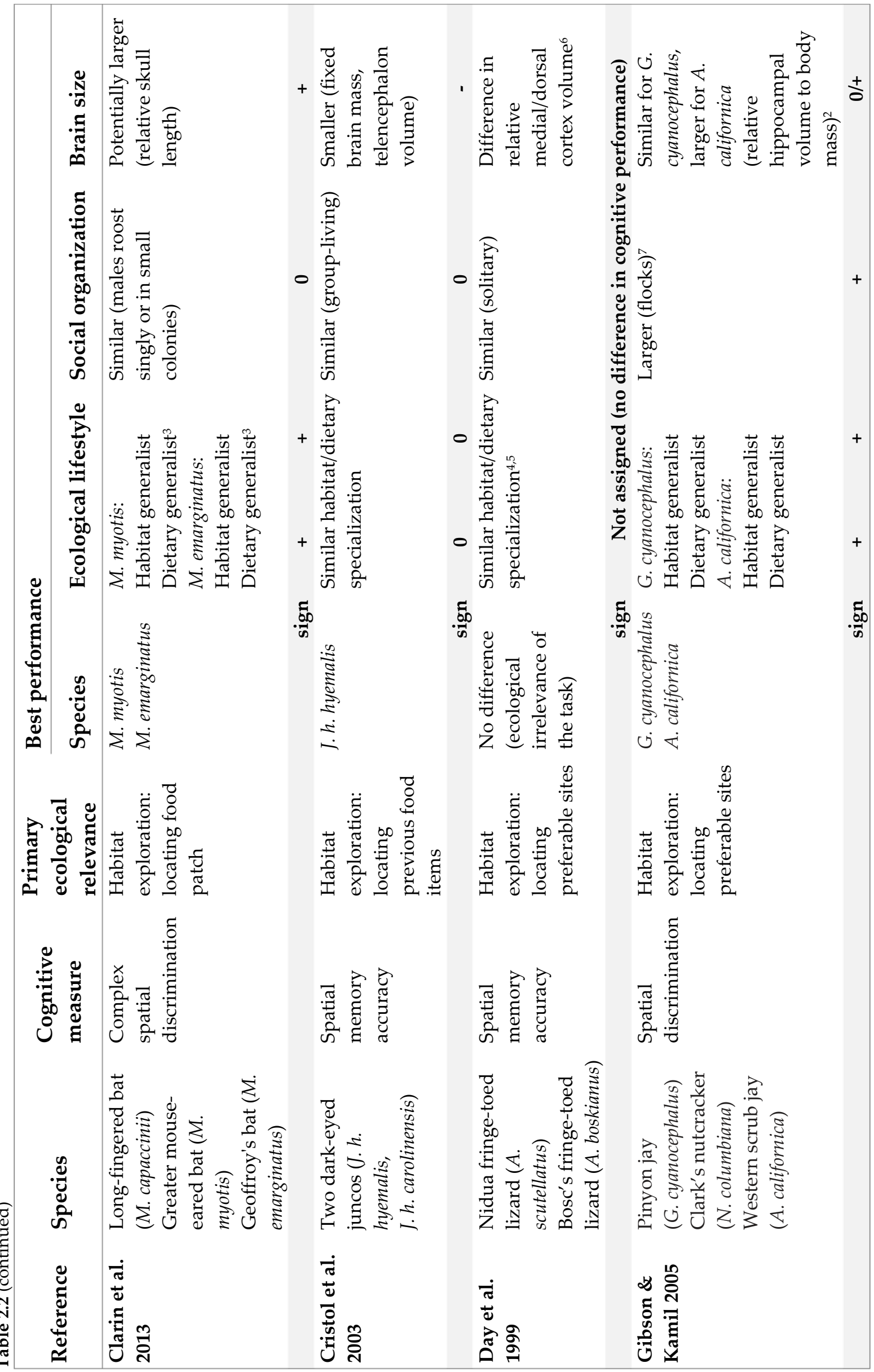




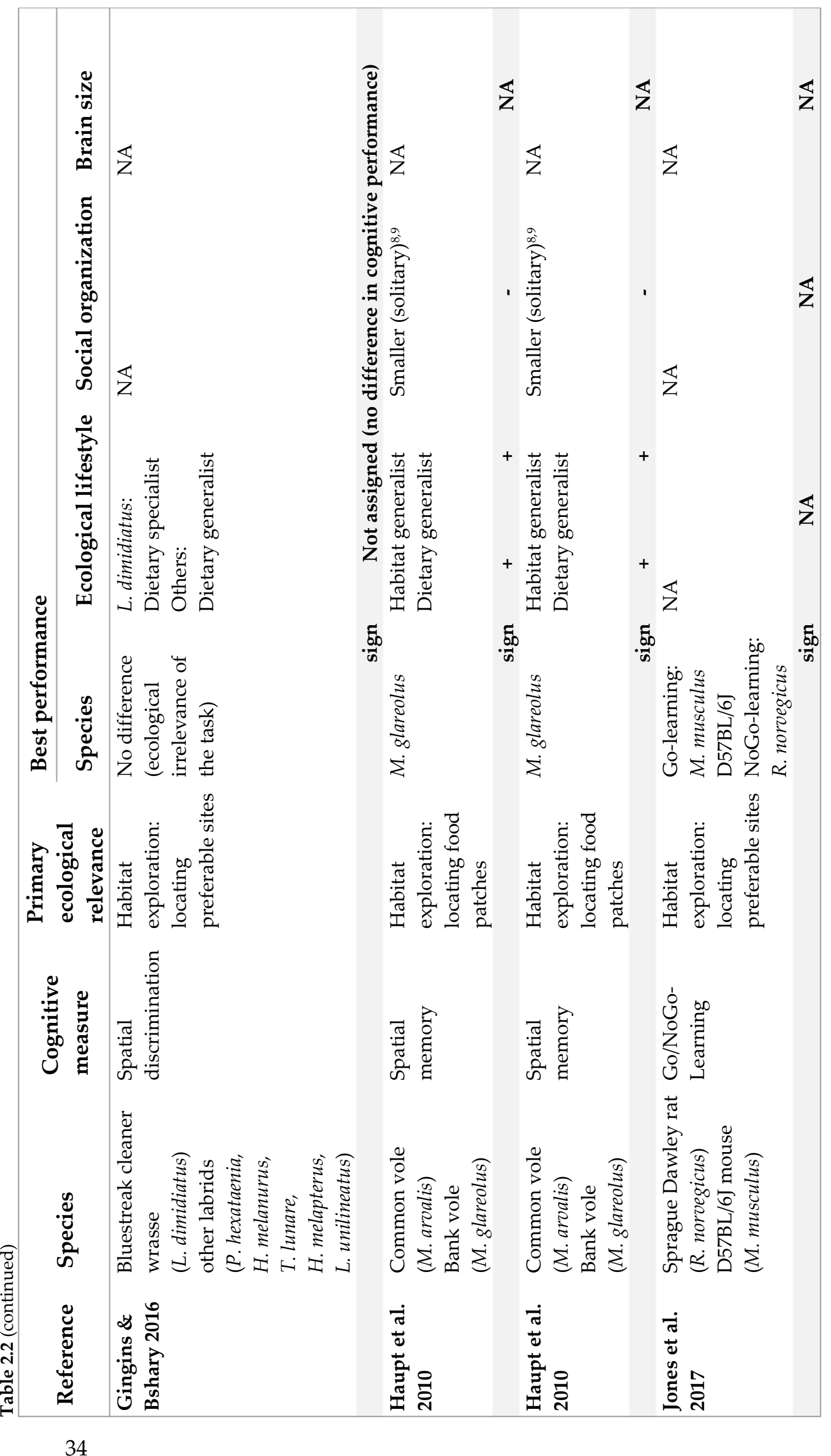




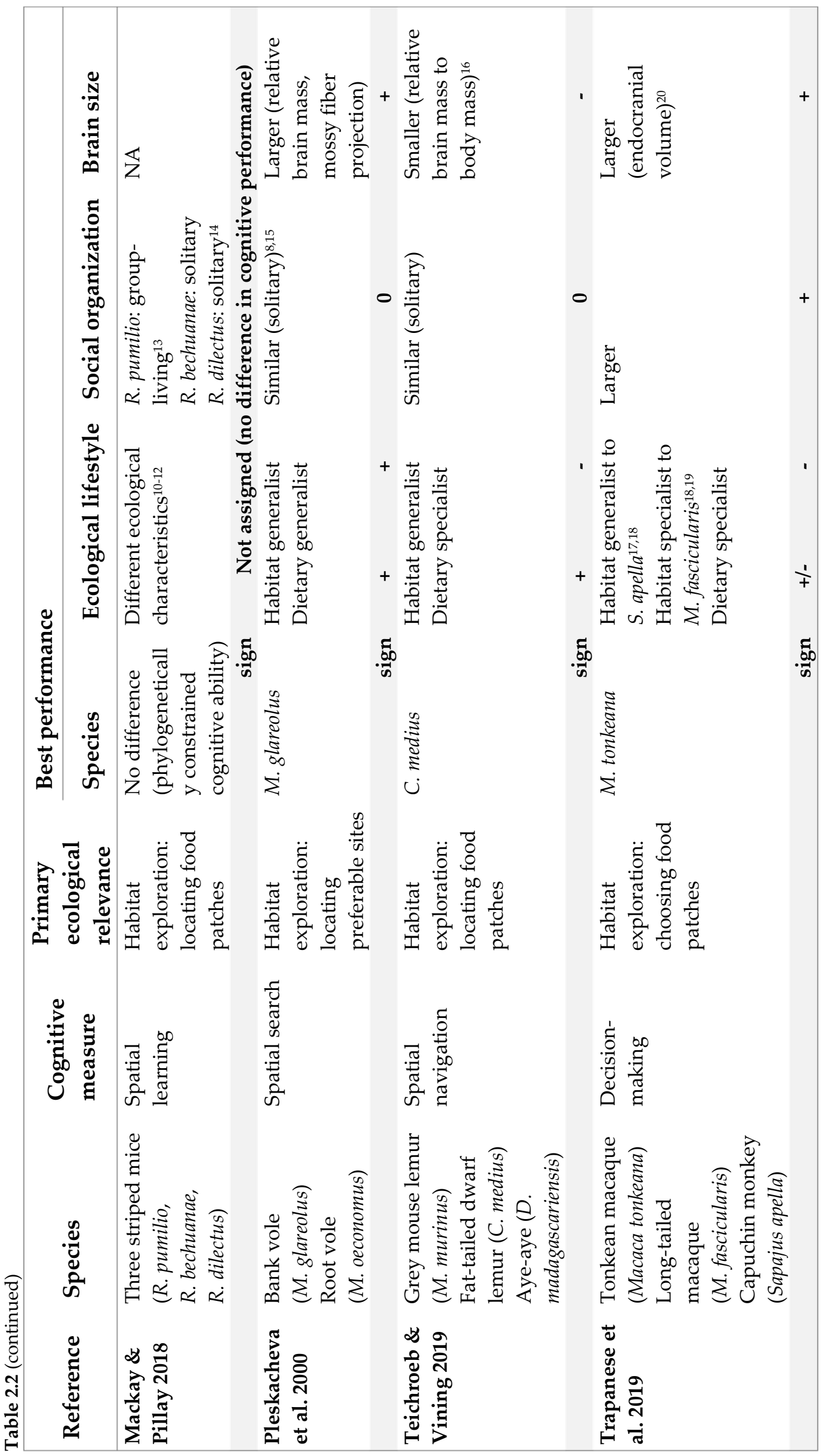




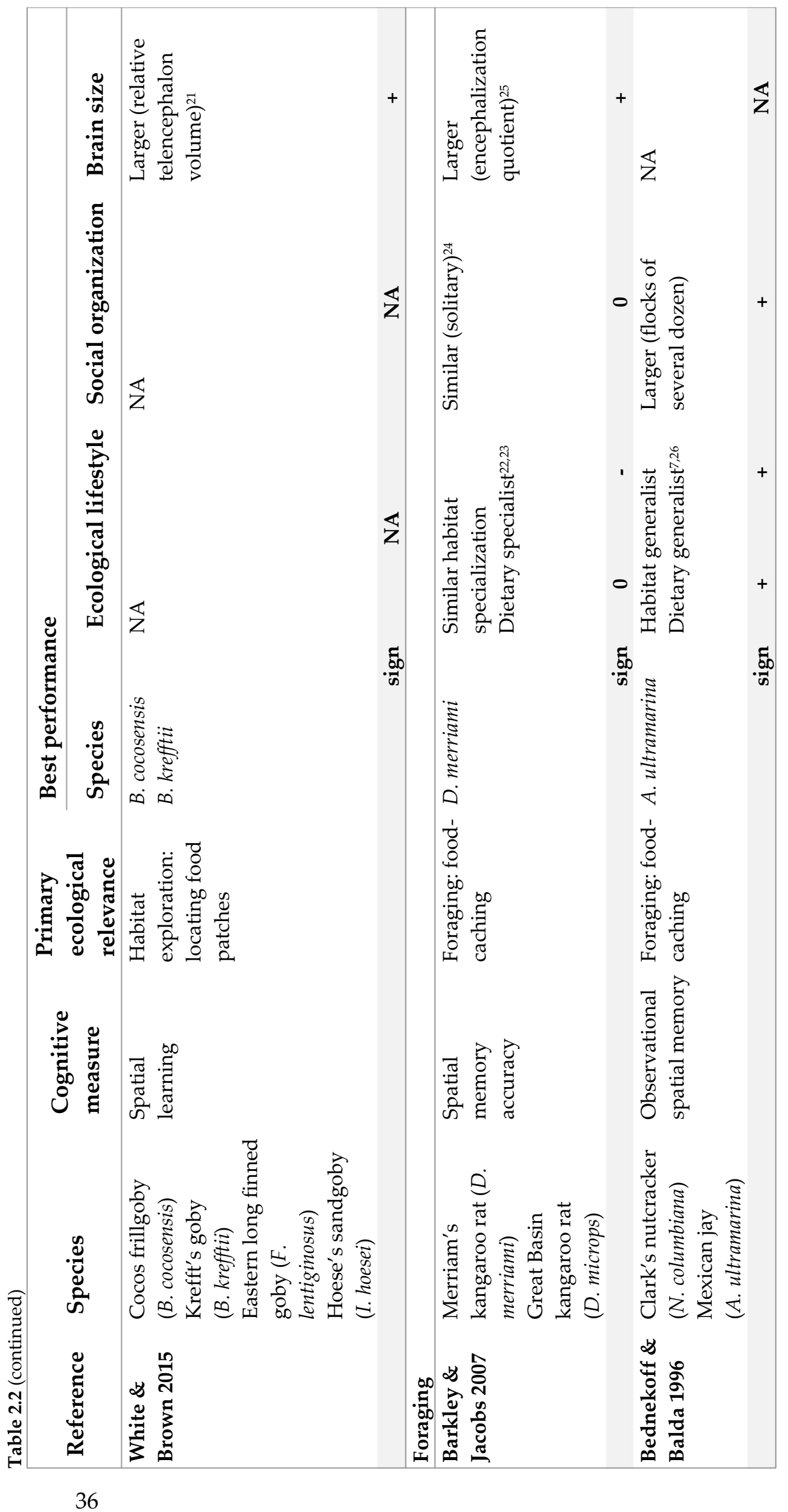




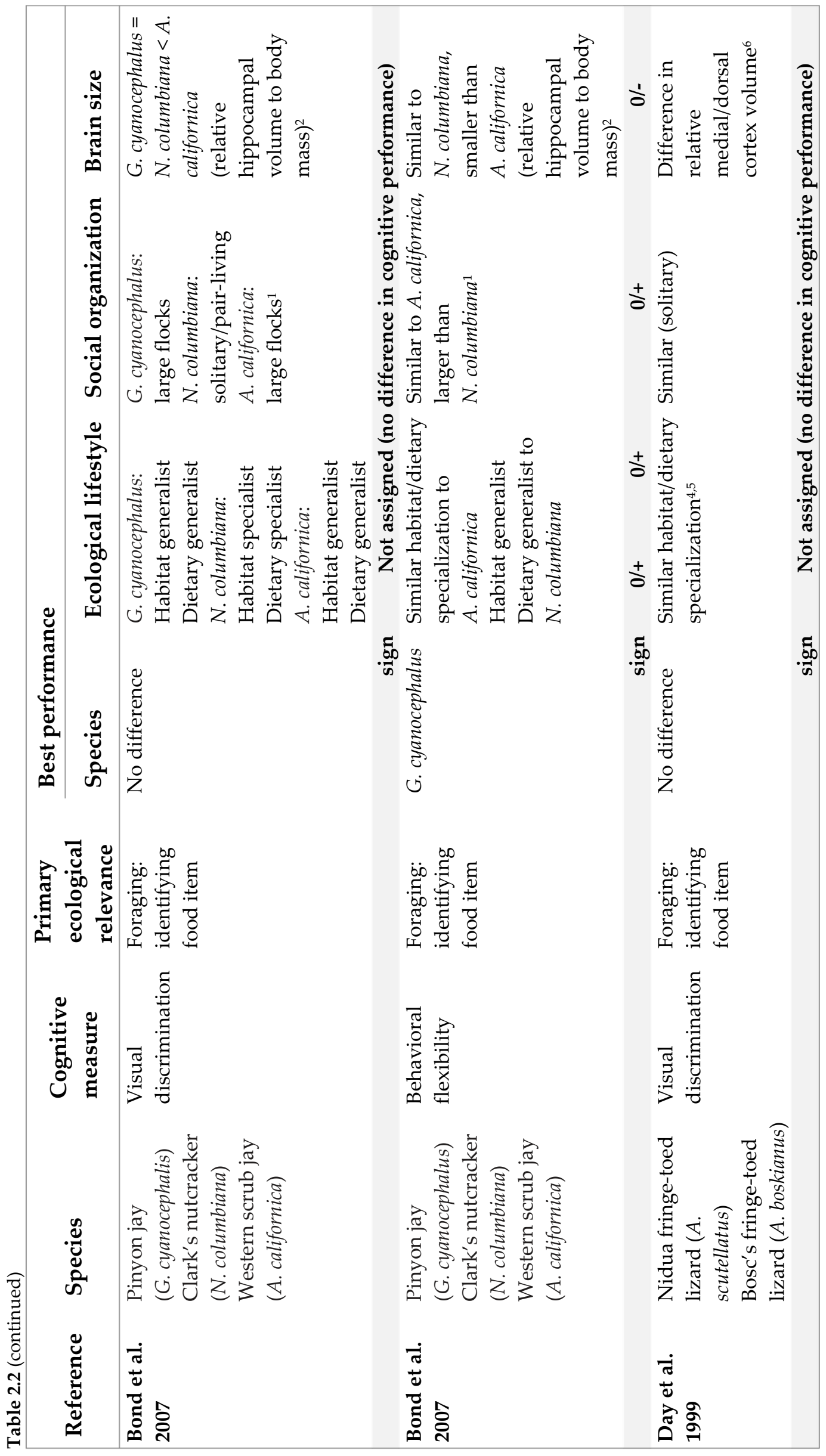




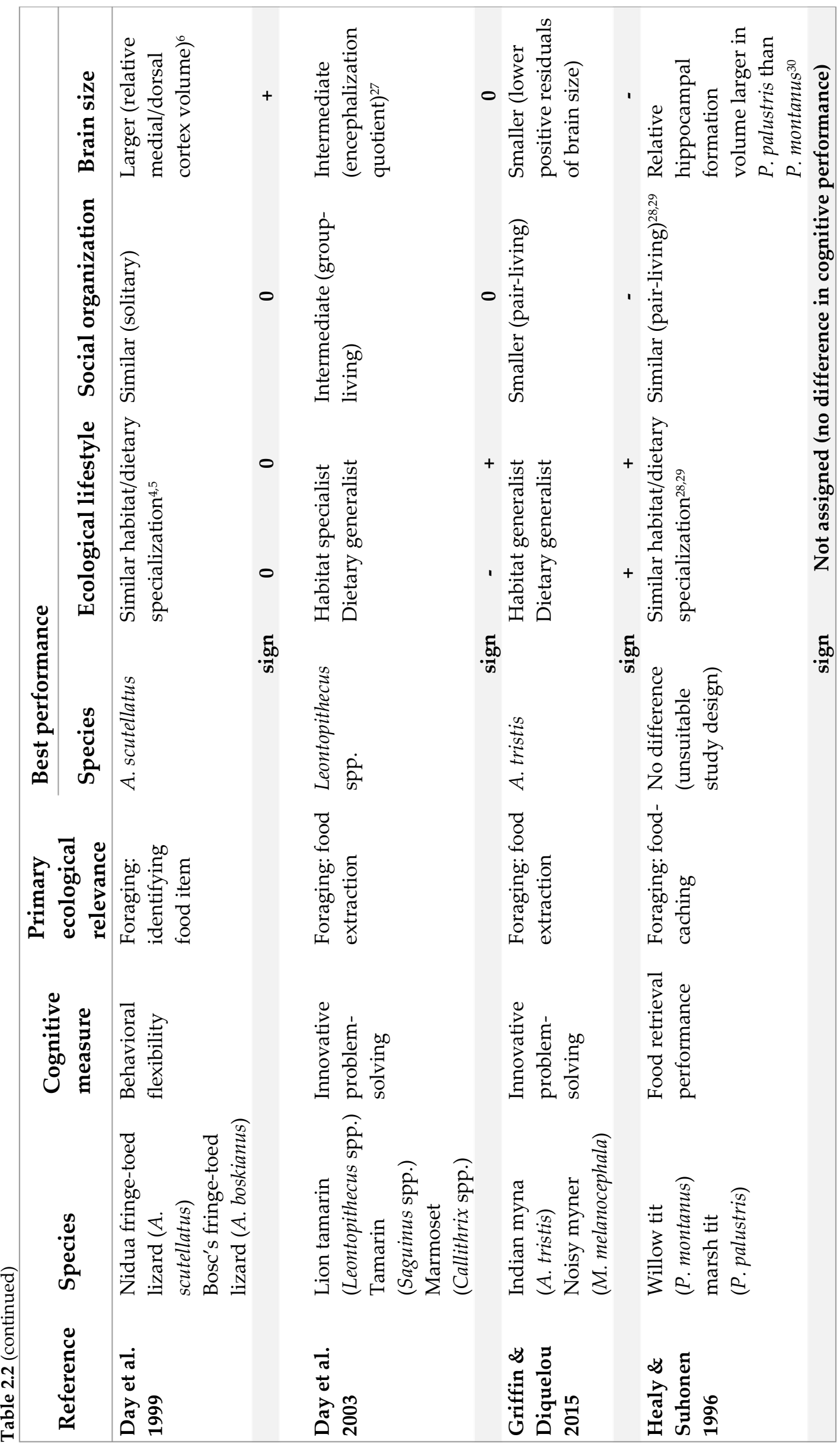




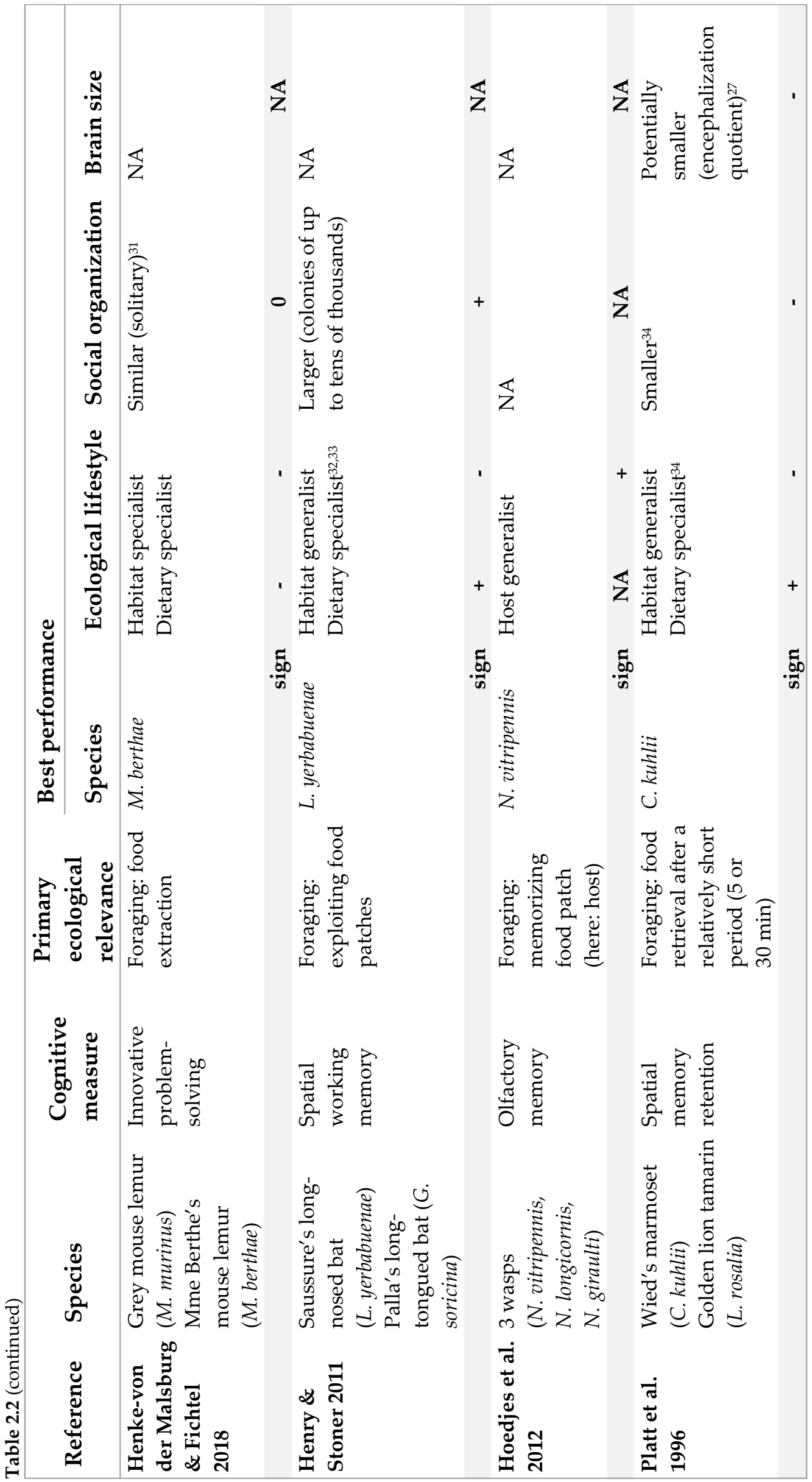


Chapter II: Literature review

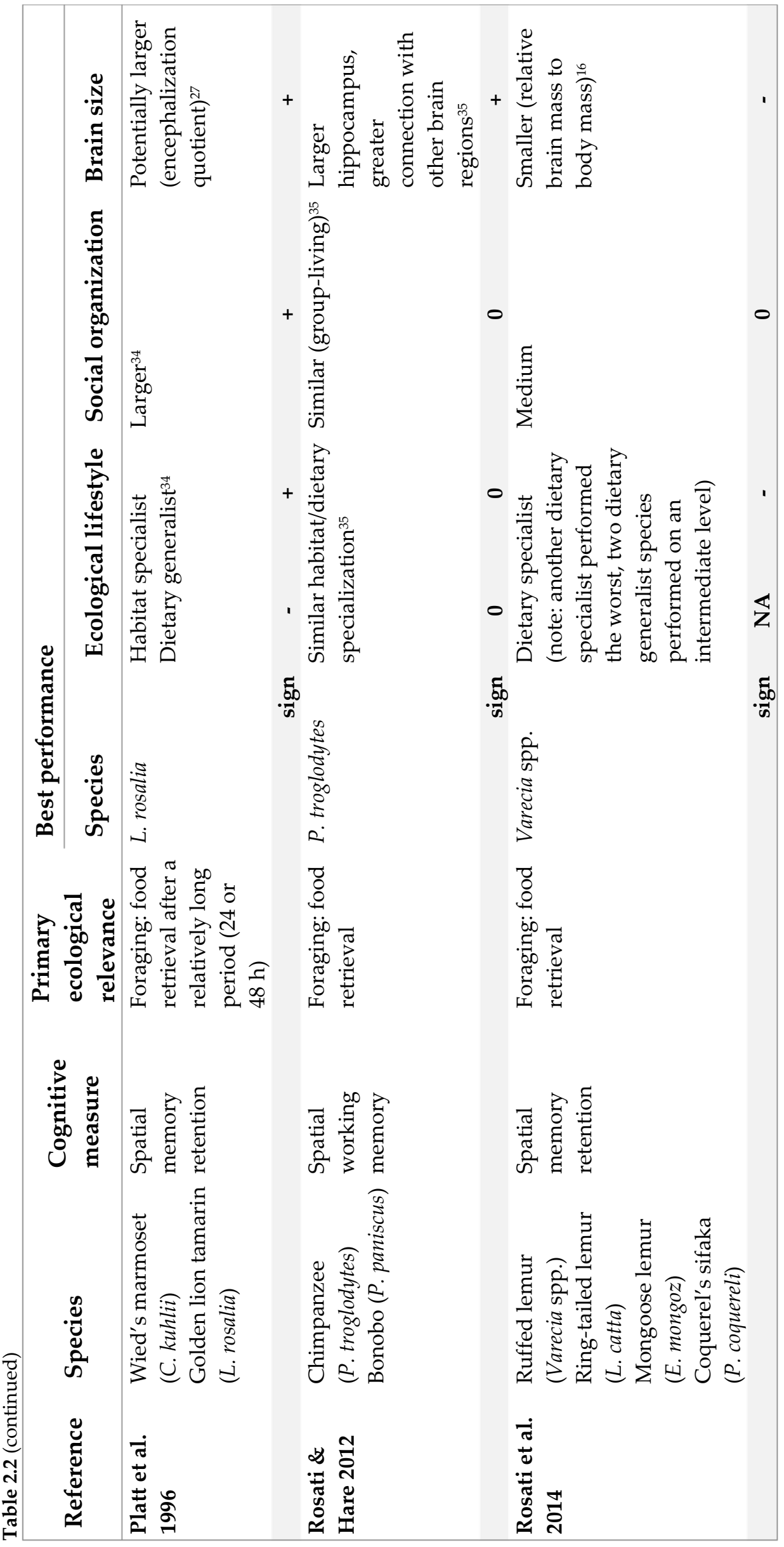




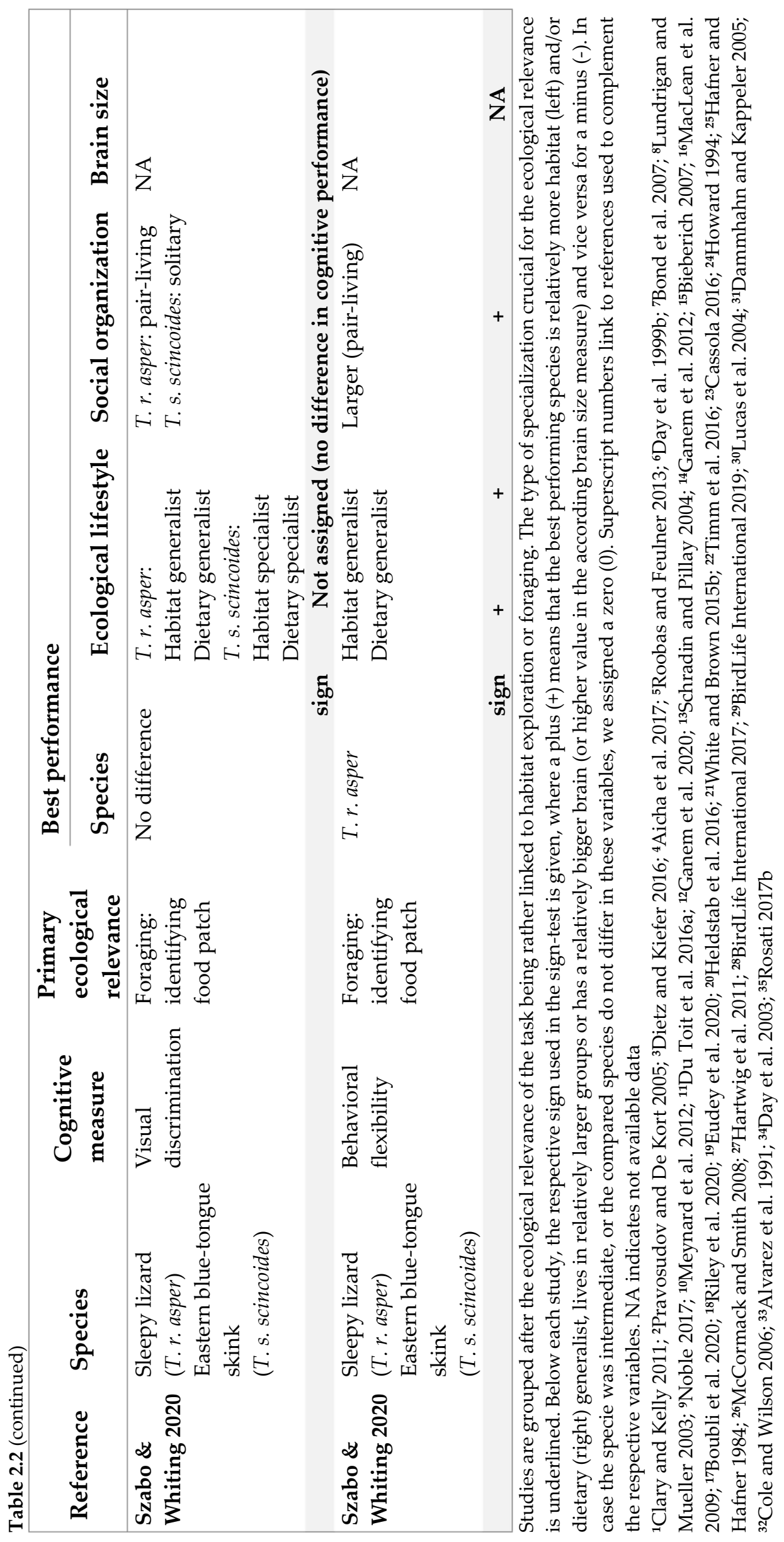


et al. 2012), or behavioral flexibility (Day et al. 1999a), using a variety of problem-solving experiments, including food extraction tasks, visual or olfactory discriminations, or reversal learning tasks.

\subsection{Habitat exploration and the degree of habitat specialization}

Occurring across larger and more complex areas, habitat generalists would benefit from advanced navigational strategies to efficiently explore these areas, specifically when they feed on patchy distributed but potentially predictable food sources. The habitat generalist bank vole, for instance, showed increased spatial memory compared to the more specialized root vole (Pleskacheva et al. 2000). In lemurs, the species with greater habitat specialization performed better in the spatial memory task due to more efficient use of navigational heuristics (Teichroeb and Vining 2019). In bats, Saussure's long-nosed bats, a relative habitat generalist, exhibited more efficient feeder exploitation than the sympatric but more specialized long-tongued bats (Henry and Stoner 2011). Similarly, the relative habitat generalists, the greater mouse-eared and Geoffroy's bats, learned a complex visual discrimination faster and were more flexible in a reversal learning task than the most specialized long-fingered bat (Clarin et al. 2013).

Migratory vertebrates tend to be more habitat specialists than generalists, possibly because they need to disperse further to find a habitat patch with suitable conditions (Martin and Fahrig 2018). In a comparative study on spatial memory accuracy in two junco subspecies (Cristol et al. 2003), the migratory subspecies performed better than the residential subspecies. However, since both subspecies rely on comparable diets and occur in similar habitats throughout the year, and due to a lack of data on population densities and different habitat types, it is neither possible to calculate a species specialization index (Martin and Fahrig 2018), nor to classify the migratory subspecies as more specialized than the residential one. Overall, one may argue, however, that the degree of habitat specialization tends to covary with spatial memory accuracy, learning flexibility and decision-making. 


\subsection{Foraging and the degree of dietary specialization}

Food storing or caching can be considered as a behavioral adaptation to variable resource abundance. While a positive link between these behaviors and spatial memory abilities has been reported (Clayton and Krebs 1994a, b; Bednekoff et al. 1997), it is not clear how the degree of ecological generalism may fit into this relationship. The aforementioned example on spatial memory accuracy in a cache simulation task reported the better performing kangaroo rat to be a leaf-eating specialist (Barkley and Jacobs 2007), but others classified them as less specialized because they also, although more rarely, feed on seeds like other kangaroo rats (Cassola 2016; Timm et al. 2016), indicating that the classification into generalists and specialists is not always straightforward.

The degree of dietary breadth was related to discriminative learning abilities in a comparative study of parasitic wasps. The more dietary generalists (Nasonia vitripennis, $N$. longicornis) learned an association between an odor and a rewarding host better than the more specialized N. giraulti (Hoedjes et al. 2012). Also, the dietary generalist bank vole performed better in a spatial learning task with temporally changing food locations than the more dietary specialist common vole (Haupt et al. 2010). Hence, dietary generalism seems to covary with discriminative and spatial learning abilities.

Individuals with greater innovative abilities are also expected to deal more efficiently with changing environmental conditions, including diet (Sol et al. 2005a). Since generalist species are by definition exposed to more variable conditions, they are suggested to express greater behavioral flexibility and greater innovative propensities than specialists (Ducatez et al. 2015; Navarrete et al. 2016). Among birds, habitat generalist species have indeed higher innovation rates than habitat specialists (Overington et al. 2011). Moreover, the omnivorous Indian myna showed a higher innovative propensity than the noisy miner, which is specialized on honey (Griffin and Diquelou 2015). In contrast, the more dietary and habitat specialized Madame Berthe's mouse lemur outperformed the sympatric generalist gray mouse lemur in innovative extractive foraging tasks with variable difficulties (Henke-von der Malsburg and Fichtel 2018). The better performance of the specialist might have been a result of enhanced executive control enabling individuals to inhibit the use of a previously learned problem-solving technique and to develop an adjusted solution to a modified problem, indicating that other factors 
than ecological specialization may explain variation in performance. Across primates, however, performance in inhibitory control tests was best predicted by absolute brain size and dietary breadth, suggesting that species differences in dietary specialization might indeed be related to levels of self-control (MacLean et al. 2014). Hence, dietary specialization seems to covary with learning ability, spatial learning, and inhibitory control.

In general, cognitive performance can be related to the degree of ecological specialization. Eighteen of the interspecific comparisons reviewed here report cognitive differences between species expressing a different degree of habitat specialization, while five do not (sign-test: $p=0.011)$. By trend, habitat generalists $(\mathrm{N}=13)$ were more likely to outperform habitat specialists $(\mathrm{N}=5$; sign-test: $p=0.064)$. Twenty-one of the comparisons report cognitive differences between species expressing a different degree of dietary specialization while three do not (sign-test: $p<0.001)$. However, the degree of dietary specialization could not explain interspecific variation in cognitive performance (sign-test: $\mathrm{N}_{\text {generalist }}=13, \mathrm{~N}_{\text {specialist }}=8, p=0.383$ ).

\section{Variation in cognitive performance in relation to group size}

Because performance in cognitive tests in these studies might be explained by consistent differences in sociality, we scored the species included in this review with respect to their group size to index their social complexity (Table 2.2). While half of the comparisons $(\mathrm{N}=12)$ controlled for group size effects, the other half $(\mathrm{N}=12)$ compared species living in differently sized groups, which also exhibit interspecific cognitive variation. However, we do not find species living in larger groups to generally perform better than those living in smaller groups, which might also be due to our small sample size (sign-test: $\mathrm{N}_{\text {larger }}=7$, $\left.\mathrm{N}_{\text {smaller }}=5, p=0.774\right)$.

Five studies supported a positive link between group size and cognitive performance: Mexican, pinyon, as well as Western scrub jays, which live in larger flocks,

performed better in spatial learning paradigms than Clark's nutcrackers (Bednekoff and Balda 1996; Gibson and Kamil 2005), Saussure's long-nosed bats, which form larger 
colonies, performed better in a spatial working paradigm than Palla's long-tongued bats (Henry and Stoner 2011), pair-living sleepy lizards were more behaviorally flexible in a visual reversal learning task than solitary Eastern blue-tongue skinks (Szabo and Whiting 2020), and golden lion tamarins, which live in relatively larger groups, showed better memory retention for longer retention intervals than Wied's marmosets (Platt et al. 1996). However, Wied's marmosets outperformed golden lion tamarins in the same task using a shorter retrieval period (Platt et al. 1996). In lemurs, performance in a spatial memory task was not linearly related to group size across four species (Rosati et al. 2014). Moreover, in innovative problem-solving paradigms, primates living in intermediate-sized (Day et al. 2003) or birds living in smaller groups (Griffin and Diquelou 2015) outperformed the respective species living in relatively larger groups.

Based on the currently available evidence, it is therefore not possible to determine whether sociality covaries with cognitive performance scores in these studies. First, we set the focus on studies comparing species with variation in ecological adaptations, which were not designed to compare species with different group size. Second, we indexed sociality in terms of group size, but disregarded group composition, stability, cohesion, or hierarchy. Finally, most of the cognitive tests were not explicitly designed to have any functional relevance in terms of sociality or variation in social traits, so that a correlation with performance scores is unlikely. Hence, to address the relative importance of either ecological or social factors driving the evolution of brain size, comprehensive cognitive test batteries addressing both sets of factors are required (Shaw and Schmelz 2017; Völter et al. 2018; Fichtel et al. 2020).

\section{Variation in cognitive performance in relation to brain size}

Brain size can be assessed via absolute or relative brain mass or volume, via an encephalization quotient (Jerison 1973; Hartwig et al. 2011), or neural connectivity, for instance. Since the studies included in this review were not designed to compare potential effects of a certain brain size measure, we had to find comparable measures or proxies elsewhere (Table 2.2). With this information, we did not find evidence for a link between experimental cognitive performance and brain size in this sample (sign-test: $N_{\text {bigger }}=10$, 
$\left.\mathrm{N}_{\text {smaller }}=7, p=0.629\right)$. Since the available brain size measures differed between these relatively few studies, the observed lack of an effect of brain size on cognitive performance might reflect this methodological shortcoming.

\section{Discussion}

In this review, we summarized comparative research investigating variation in cognitive performance in relation to specific adaptations to ecological factors animals are exposed to in their daily life. Although these ecological factors can vary greatly among species in the same taxonomic group, studies systematically investigating the relationship between ecological factors and cognitive performance are still rare. Most studies reported a predicted relationship between the measured cognitive performance and an ecological factor differentiating the study species (Table 2.1), but we cannot know whether this pattern is affected by a publication bias against studies reporting no effect. Our rough control indicated that phylogeny as well as group and brain size did not have pervasive effects on the observed pattern. Nonetheless, some studies failed to find an effect of ecology, perhaps due to unsuitable study designs (Bednekoff and Balda 1996; Healy and Suhonen 1996) and/or ecological irrelevance of the respective cognitive ability tested (Day et al. 1999a; Gingins and Bshary 2016). Below, we discuss the main correlates of interspecific variation in cognitive performance in terms of flexible foraging strategies, spatiotemporal habitat exploration and food patch exploitation, as well as the degree of ecological specialization. Further, we highlight the importance of considering potentially confounding factors when designing a study appropriate for the investigation of speciesspecific ecological adaptations (Shaw and Schmelz 2017; Schubiger et al. 2020).

\subsection{Adaptations to ecological factors correlate with cognitive performance}

\subsubsection{Flexible foraging strategies}

Interspecific differences in cognitive performance have been reported as a function of variation in ranging behavior and search strategies (Trapanese et al. 2019; Teichroeb and 
Vining 2019), foraging activity (Day et al. 1999a; Day et al. 2003; Jones et al. 2017), foraging techniques (Day et al. 2003), characteristics of preferred dietary items (Pleskacheva et al. 2000; Henry and Stoner 2011; Teichroeb and Vining 2019), adaptations to habitat complexity (Pleskacheva et al. 2000; Clarin et al. 2013; White and Brown 2015a), or adaptations to seasonality (Cristol et al. 2003; Barkley and Jacobs 2007; Henry and Stoner 2011) (Fig. 2.2). Active or manipulative extractive foragers have been reported to learn more flexibly (Day et al. 1999a) or to be more innovative (Day et al. 2003), respectively. Flexible learning and innovative abilities can be linked in innovators that possess the ability to invent a new behavior or to modify an existing behavior (Reader and Laland 2003) and to incorporate these into the behavioral repertoire via flexible learning

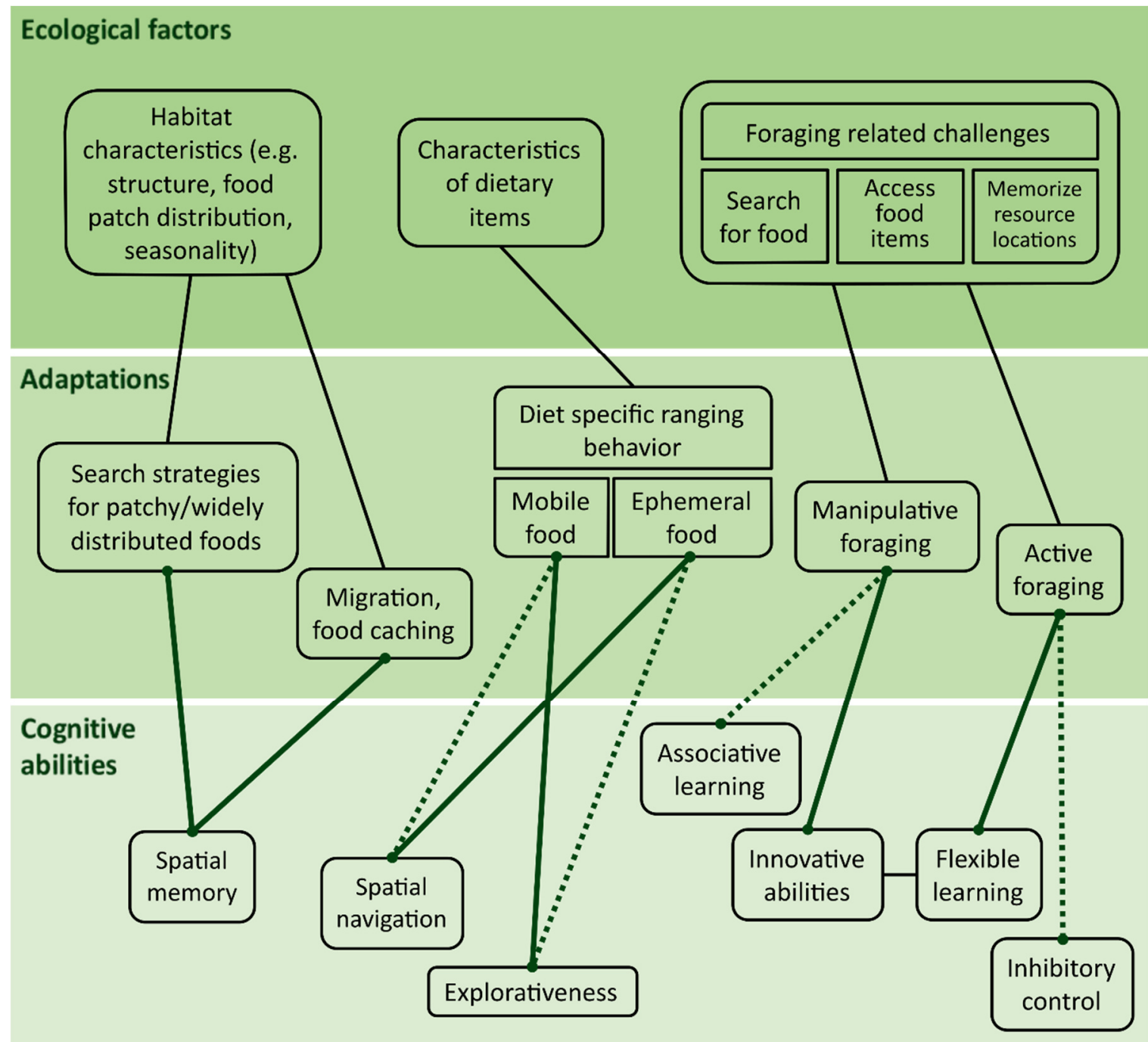

Fig. 2.2 Links between ecology and cognition. We identified several links (thick lines) between cognitive abilities and adaptations to ecological factors (thin lines) such as certain habitat characteristics, characteristics of dietary items or ecological challenges related to the foraging process (including the search for food, access to food items and memorizing resource locations). Positive relationships between specific adaptations and cognitive abilities are represented with thick solid lines while negative relationships are represented with dashed lines 
mechanisms (Dukas and Ratcliffe 2009). In contrast to sit-and-wait predators, active foragers need to rapidly adjust the current foraging strategy to the behavior of the prey of interest as well as to the environment while searching and hunting for prey. They would specifically benefit by learning the association of a certain stimulus with a reward (i.e., the prey) and to flexibly update such associations whenever the stimulus or other conditions change. This underlying behavioral flexibility is then an advantageous characteristic when environmental conditions change (Lee 2003; Lefebvre et al. 2004). Further, innovations appear predominantly in the foraging context (Reader and MacDonald 2003), favoring a link with a species' foraging ecology rather than with its sociality.

\subsubsection{Spatiotemporal habitat exploration and food patch exploitation}

When searching for food, animals always need to deal with the trade-off between habitat exploration and food patch exploitation (Hills et al. 2015). The decision of when to switch from one to the other varies with the spatiotemporal distribution of food items since this determines the energetic costs of habitat exploration and energy intake during patch exploitation. Habitat exploration is particularly costly when food items are sparse or patchy distributed or when food abundance is (seasonally) low. Using specific navigational heuristics or investment in spatial memory can reduce foraging costs under these conditions. However, the underlying capacities are energetically constrained and should, therefore, evolve in species that feed on stationary rather than mobile food items, on dispersed rather than highly abundant items, or in species, that are exposed to harsh environments (Roth and Pravosudov 2009).

Also, when relocating a certain food patch, irrespective of the length of a retrieval interval, the energetic investment in spatial memory capacities can be beneficial. At least in birds, rodents, and primates, there is evidence that the evolution of spatial memory abilities parallels a species-specific foraging ecology. Better spatial cognition has been reported for species feeding on dispersed items of rather unpredictable abundance (Platt et al. 1996; Pleskacheva et al. 2000; Rosati and Hare 2012; Clarin et al. 2013), frugivorous species (Rosati et al. 2014; Trapanese et al. 2019; Teichroeb and Vining 2019), scatter hoarders (Barkley and Jacobs 2007) or migrating species (Cristol et al. 2003). In such cases, 
better-adapted species evolve greater hippocampi as an adaptation to the highly demanding ecological challenge of memorizing previous food locations or caches.

\subsubsection{Other ecological adaptations}

Evidence for a general association between cognitive performance and ecological factors might be biased since most studies measuring interspecific variation conducted experiments on only a few cognitive skills such as spatial processing, flexible learning or innovative problem-solving. It remains to be investigated, however, whether other cognitive tasks, such as those estimating the ability of causal reasoning or numerical understanding, or a combination of different tasks in a valid test battery can also be linked to ecological factors (Shaw and Schmelz 2017). Also, the potential effects of other ecological factors, such as predation and parasite risk, on relevant cognitive abilities remains largely unstudied (Garamszegi et al. 2007; Soler et al. 2012). Thus, there is a need for additional studies to obtain a more comprehensive understanding of the ecologycognition link.

\subsection{How does cognitive performance correlate with the degree of ecological specialization?}

In several studies we found the degree of ecological specialization to be correlated with variation in certain cognitive abilities (Platt et al. 1996; Pleskacheva et al. 2000; Day et al. 2003; Barkley and Jacobs 2007; Haupt et al. 2010; Henry and Stoner 2011; Hoedjes et al. 2012; Rosati et al. 2014; Griffin and Diquelou 2015; Henke-von der Malsburg and Fichtel 2018). We found that habitat generalists were likely to outperform habitat specialists, but this relation was not significant and the degree of dietary specialization did not consistently covary with cognitive performance. Given, that habitat generalists regularly face more challenges by exploring a broader, more variable habitat than specialists, they might specifically exhibit better spatial processing abilities and more behavioral flexibility (Overington et al. 2011); at least in the sense of 'behavioral flexibility' allowing for adaptations to variable environments (Lea et al. 2020). However, behavioral flexibility 
does not necessarily result in better cognitive performance per se because less behaviorally flexible species, as specialists, may instead possess other behavioral characteristics that promote better performance in certain cognitive skills (Henke-von der Malsburg and Fichtel 2018). In studies reporting the more specialized species to exhibit better performance than the generalist (e.g. Teichroeb and Vining 2019), better performance was linked to specific adaptations for habitat exploration or exploitation. In this context, efficiency can be of various nature: to not use certain paths multiple times while foraging, to deplete a certain food patch to a certain extent varying with search decision rules (Wilke et al. 2009), or to not use ineffective solutions but to inhibit the execution of related behaviors. Greater efficiency might be more likely to vary with specific adaptations to certain ecological challenges than to the degree of ecological specialization per se and would require a more detailed investigation than the currently available evidence allows.

\subsection{Confounding factors and limitations of the review}

Several factors may confound a putative relationship between a particular ecological factor and a corresponding cognitive ability. First, the cognitive trait under study must have ecological relevance. This problem becomes apparent for example in a study designed to compare cognitive abilities in an ecologically non-relevant context, i.e., spatial discrimination abilities across six species of labrid fishes (Gingins and Bshary 2016). Because these cleaner fish do not rely on advanced spatial abilities, as they do not actively search for food patches but are visited by parasitized client fish, it is not surprising that they exhibited similar performances when associating a food source with a location.

Second, social factors that differ systematically between species may obscure the effects of ecological factors on cognitive abilities. For example, more social Mexican jays performed better than food-caching Clark's nutcrackers in a spatial memory task in which the birds' recovery performance of food caches made by conspecifics was measured (Bednekoff and Balda 1996). Similarly, of three corvid species, pinyon jays, who live in the most socially complex environment, performed better in a reversal learning task than Clark's nutcrackers and Mexican jays with a more generalist ecology (Bond et al. 2007). 
Finally, given the currently available sample size of studies meeting our criteria, the purported link between ecological specialization and cognitive performance might reflect a publication bias. Comparisons of mostly mammals (especially primates) and birds, and the focus on ecological challenges related to foraging may also create a bias. Nonetheless, we hope that the present review will stimulate more comparative research regarding this interesting topic, using additional taxa and, most importantly, a wider range of ecologically relevant cognitive traits to draw firm conclusions about the relative importance of various ecological factors in shaping cognitive abilities.

\section{Acknowledgments}

We are thankful for constructive comments by two anonymous reviewers and thank TRC Illing for discussions. 


\section{Appendix 1}

Table S1-1 Definitions and explanations of cognitive terms.

\begin{tabular}{|c|c|}
\hline \multicolumn{2}{|l|}{ General terms } \\
\hline Cognitive ability & $\begin{array}{l}\text { Ability to perceive, process and memorize information from the } \\
\text { environment or other individuals. }\end{array}$ \\
\hline Decision-making & $\begin{array}{l}\text { Determination of an action considering environmental cues and } \\
\text { experience. }\end{array}$ \\
\hline Learning & $\begin{array}{l}\text { Any change in behavior as result of experience; operationalized by } \\
\text { an increase in performance. }\end{array}$ \\
\hline $\begin{array}{l}\text { Cognitive task } \\
\text { performance }\end{array}$ & $\begin{array}{l}\text { Operationalization for an individual's learning abilities, often } \\
\text { measured as success latency or ratio to solve a problem. }\end{array}$ \\
\hline $\begin{array}{l}\text { Problem-solving } \\
\text { ability }\end{array}$ & $\begin{array}{l}\text { Ability to solve problems incorporating objects (i.e., inanimate } \\
\text { objects, food), e.g., puzzle box, object manipulation test. }\end{array}$ \\
\hline $\begin{array}{l}\text { General intelligence } \\
\text { (g-factor) }\end{array}$ & $\begin{array}{l}\text { Composite factor derived from correlating cognitive performances } \\
\text { across various cognitive tasks. }\end{array}$ \\
\hline $\begin{array}{l}\text { Cognitive test } \\
\text { battery }\end{array}$ & $\begin{array}{l}\text { Array of at least two different cognitive tasks; often conducted to } \\
\text { investigate correlations between cognitive abilities. }\end{array}$ \\
\hline \multicolumn{2}{|c|}{ Cognitive abilities related to perception } \\
\hline $\begin{array}{l}\text { Discrimination } \\
\text { learning }\end{array}$ & $\begin{array}{l}\text { Learning contingencies between events via conditioning. In a visual } \\
\text { discrimination learning task, usually objects differing in shape, color, } \\
\text { pattern, and/or location serve as stimuli, of which only one is } \\
\text { associated with a reward. Performance is measured as trials or time } \\
\text { until a specified learning criterion is reached. }\end{array}$ \\
\hline Reversal learning & $\begin{array}{l}\text { Reversed learning after an initial discrimination learning with the } \\
\text { previously rewarded stimulus becoming unrewarded. }\end{array}$ \\
\hline Numerical learning & $\begin{array}{l}\text { Learning of contingencies in relation with numbers, e.g., the ability } \\
\text { to discriminate between quantities. }\end{array}$ \\
\hline \multicolumn{2}{|c|}{ Cognitive abilities related to processing } \\
\hline Behavioral flexibility & $\begin{array}{l}\text { Ability to flexibly adjust the behavior according to the actual } \\
\text { circumstances. }\end{array}$ \\
\hline Innovation & $\begin{array}{l}\text { Solution to a novel problem or novel solution to a modified problem; } \\
\text { operationalized using problem-solving tasks. }\end{array}$ \\
\hline Causal reasoning & $\begin{array}{l}\text { Cognitive ability to relate two events with each other using causal } \\
\text { understanding instead of arbitrary contingencies like space or time; } \\
\text { operationalized using, e.g., a string-pulling task where pulling a } \\
\text { string should be related to getting access to an attached reward. }\end{array}$ \\
\hline Tool use & $\begin{array}{l}\text { Behavior in which an animal uses a secondary object as a specific } \\
\text { tool to solve a given problem. }\end{array}$ \\
\hline
\end{tabular}


Table S1-1 (continued)

\begin{tabular}{|c|c|}
\hline \multicolumn{2}{|c|}{ Cognitive abilities related to memory } \\
\hline Shor-term memory & $\begin{array}{l}\text { Holding information that is currently being processed, e.g., the } \\
\text { memorization of a certain learning contingency from one } \\
\text { experimental session to the other. }\end{array}$ \\
\hline Long-term memory & $\begin{array}{l}\text { Relatively persistent storing of information; can be manifested via } \\
\text { several repetitions. }\end{array}$ \\
\hline Spatial memory & $\begin{array}{l}\text { Holding specifically spatial information; operationalized, e.g., using } \\
\text { a maze with several end locations of which only one holds the } \\
\text { rewarded stimulus, or using a cache simulation task, where the } \\
\text { subject can relocate a hidden food reward after a certain retention } \\
\text { interval. }\end{array}$ \\
\hline \multicolumn{2}{|c|}{ Cognitive abilities in social contexts } \\
\hline Social learning & $\begin{array}{l}\text { Changes in an individual's behavior resulting from attending to } \\
\text { another individual's behavior or its products. }\end{array}$ \\
\hline Recognition memory & Memory capacities to recognize other individuals. \\
\hline Transitive inference & $\begin{array}{l}\text { Deductive reasoning allowing to derive a relation between items or } \\
\text { individuals that have not been explicitly compared before. I.e., if A is } \\
\text { related to B and B is related to C, then A must also be related to C. }\end{array}$ \\
\hline Tactical deception & $\begin{array}{l}\text { Usage of a behavior in an unusual context that is likely to be } \\
\text { misinterpreted by other individuals. }\end{array}$ \\
\hline \multicolumn{2}{|c|}{ Individual characteristics that could affect problem-solving performance } \\
\hline Personality trait & $\begin{array}{l}\text { Behavior that is consistent across time and space. } \\
\text { E.g., exploration as the tendency to explore an unknown arena over } \\
\text { a short time interval. }\end{array}$ \\
\hline Persistence & $\begin{array}{l}\text { Perseverance of a certain behavior despite its inefficiency; often } \\
\text { operationalized by measuring the manipulative effort towards a } \\
\text { puzzle box with a blocked opening mechanism. }\end{array}$ \\
\hline $\begin{array}{l}\text { Executive/Inhibitory } \\
\text { /Self-control }\end{array}$ & $\begin{array}{l}\text { Ability to inhibit a prepotent behavior due to its (temporary) } \\
\text { inefficiency; operationalized using, for example, a detour reaching } \\
\text { task with a transparent cylinder. }\end{array}$ \\
\hline
\end{tabular}

\section{Electronic supplementary material}

The online version contains the literature search records as supplementary material and is available at https://doi.org/10.1007/s00265-020-02923-Z 


\section{Chapter III: Empirical study}

\section{Linking cognition to ecology in wild sympatric mouse lemur species}

Johanna Henke-von der Malsburg, ${ }^{1,2,3}$, Peter M. Kappeler ${ }^{1,2}$, Claudia Fichtel1,3

${ }^{1}$ Behavioral Ecology and Sociobiology Unit, German Primate Centre, Leibniz Institute for Primatology, Göttingen, Germany

${ }^{2}$ Department of Sociobiology/Anthropology, Johann-Friedrich-Blumenbach Institute of Zoology and Anthropology, Georg-August-University Göttingen, Germany

${ }^{3}$ Leibniz ScienceCampus 'Primate Cognition', Göttingen, Germany

This manuscript is accepted for publication in

Proceedings of the Royal Society B: Biological Sciences (2021)

Author contributions: JHM, CF and PMK conceived the study. JHM was primarily responsible for data collection and data analyses and drafted the manuscript. All authors contributed to manuscript writing. 


\section{Abstract}

Cognitive abilities covary with both social and ecological factors across animal taxa. Ecological generalists have been attributed with enhanced cognitive abilities, but which specific ecological factors may have shaped the evolution of which specific cognitive abilities remains poorly known. To explore these links, we applied a cognitive test battery (two personality, ten cognitive tests; $\mathrm{N}=1,104$ tests) to wild individuals of two sympatric mouse lemur species ( $\mathrm{N}=120$ Microcebus murinus, $\mathrm{N}=34 \mathrm{M}$. berthae) varying in ecological adaptations but sharing key features of their social systems. The habitat and dietary generalist gray mouse lemurs were more innovative and exhibited better spatial learning abilities; a cognitive advantage in responding adaptively to dynamic environmental conditions. The more specialized Madame Berthe's mouse lemurs were faster in learning associative reward contingencies; providing relative advantages in stable environmental conditions. Hence, our study revealed key cognitive correlates of ecological adaptations and indicates potential cognitive constraints of specialists that may help explain why they face a greater extinction risk in face of current environmental changes.

\section{Background}

The evolution of cognitive abilities has been linked to variation in brain size, which covaries across species with social factors (Social Intelligence Hypothesis; Humphrey 1976) and/or ecological challenges (Ecological Intelligence Hypothesis; Parker and Gibson 1977). Recent comparative analyses across primates suggested that evolutionary variation in brain size is better predicted by ecological than social factors (DeCasien et al. 2017). Yet, little is known about whether and how these factors are linked to performance in cognitive tests in primates, but also across other taxonomic groups (Ashton et al. 2018b; Henke-von der Malsburg et al. 2020). Hence, to better understand the evolution of cognitive abilities and the underlying variation in brain size, studies of how variation in specific ecological or social factors are linked to performance in cognitive tests across taxa are required.

In this context, the degree of ecological specialization has been suggested to covary with cognitive abilities (Opportunistic Intelligence Hypothesis; Parker 1978). This notion builds upon the idea of characterizing a species' ecological niche as a multidimensional 
space that combines all adaptations to ecological conditions that contribute to its evolutionary success (Hutchinson 1953). Accordingly, an ecological generalist experiences a wider niche breadth than a specialist (Sargeant 2007).

Generalist species are assumed to be better and more flexible learners than specialists (Overington et al. 2011; Mettke-Hofmann 2014). Since generalists should be exposed to a greater variety of ecological conditions, they may also face a greater variety of ecological problems. Hence, they may have evolved specific innovative problemsolving abilities to overcome various problems. Similarly, the diverse ecological conditions may create a need for greater behavioral flexibility, especially, when conditions change unexpectedly(Lea 2020). Hence, species that experience harsher or more dynamic environmental conditions are more flexible or more innovative than others (Tebbich et al. 2016). Innovative abilities and flexibility, therefore, appear to be tightly linked (Griffin 2016). Furthermore, innovation appears to be positively correlated with other cognitive abilities (Reader et al. 2011). In addition, more innovative or behaviorally flexible species experience greater colonization success(Sol et al.2002) or a greater diversification potential (Nicolakakis et al. 2003). Both evolutionary processes have been linked to the evolution of larger brains, especially when colonizing seasonal regions (Ducatez et al. 2020b). Finally, dietary generalists have indeed larger brains than dietary specialists (Lefebvre et al. 2004; Sol et al. 2005b; MacLean et al. 2014). Despite these suggestive links, generalists, do not consistently perform better in cognitive tests than specialists, however (Henke-von der Malsburg et al. 2020).

To systematically examine covariation between cognitive abilities and the degree of ecological specialization, we applied a comprehensive cognitive test battery to wild individuals of two mouse lemur species (Microcebus spp.) that vary in some of their ecological adaptations but share key features of their social systems. Gray (M. murinus, GML) and Madame Berthe's mouse lemurs (M. berthae, MBML) represent separate lineages within the genus Microcebus that shared a common ancestor as long as 9-10 MA ago (Yoder et al. 2016). The comparison of these two species is informative because they are both nocturnal solitary foragers that are syntopic, and therefore experience identical current environmental conditions, but MBML is ecologically more specialized (Dammhahn and Kappeler 2005; Dammhahn and Kappeler 2008b). Such direct 
comparisons of cognitive performance in pairs of sympatric sister species can help to reveal the role of ecological factors in the evolution of cognition.

GML inhabit various habitat types across Western Madagascar, occur in primary as well as secondary forests, and even in highly degraded forest fragments (Lahann et al. 2006; Andriatsitohaina et al. 2020), making them habitat generalists. Their feeding niche breadth, based on Levin's standardized index, has been estimated as 0.63 (Dammhahn and Kappeler 2008b), supporting this classification. MBML occur only in a few $\mathrm{km}^{2}$ of seasonally dry deciduous lowland forests (Schäffler and Kappeler 2014) and have an annual feeding niche breadth of 0.12 (Dammhahn and Kappeler 2008b), qualifying them as habitat specialists. As the smallest living primates, they are also more sensitive to natural and anthropogenic habitat modifications (Schäffler and Kappeler 2014), markedly decreasing their population size in recent years (Schäffler et al. 2021), and justifying their classification as "Critically Endangered" (Markolf et al. 2020).

Using a comprehensive test battery with ten cognitive tests and two standard personality tests, we compared cognitive abilities of these two species. In a total of 1,104 tests, we tested $\mathrm{N}=120 \mathrm{GML}$ and $\mathrm{N}=34 \mathrm{MBML}$. As ecologically relevant abilities, we chose variation in exploration and neophilia, innovative propensities, persistence, learning abilities regarding associative and flexible learning using visual and spatial cues, and spatial memory. To also examine cognitive performances in tasks without obvious ecological relevance (Henke-von der Malsburg et al. 2020), i.e., cognitive abilities that are not expected to covary with the degree of ecological specialization, we assessed variation in inhibitory control, means-end understanding and goal directedness (see Appendix 2 for justification and predictions, Fig. 3.1). Finally, we examined whether cognitive performance across tests loads onto one common general intelligence (G/g-)factor (Humphreys 1979; Deaner et al. 2006).

\section{Materials and Methods}

\subsection{Study site and study period}

We conducted this study in Kirindy Forest, a dry deciduous lowland forest in central western Madagascar within a 12,500 ha forest concession operated by the Centre National 
de Formation, d'Etudes et de Recherche en Environnement et Foresterie (CNFEREF) Morondava. Mouse lemurs at Kirindy Forest have been captured on a monthly basis as part of an ongoing long-term project (Dammhahn and Kappeler 2005; Kraus et al. 2008). We captured GML $(\mathrm{N}=120)$ in a population that has been regularly monitored since 1993 (Eberle and Kappeler 2002) and MBML $(\mathrm{N}=34)$ in another population that has been monitored since 2002 (Dammhahn and Kappeler 2005) (see Appendix 2 for the details of the capture procedure). Between 2017 and 2019, we conducted experiments with wild animals in temporary short-term captivity across three field seasons covering the transitions from the wet to the dry season (March - May/June) and the transitions from the dry to the wet season (August - October/November), respectively.

\subsection{Study animals: Housing and experimental test battery}

In the following, we briefly describe the experimental procedure and the general statistical analyses. Detailed information about sample sizes, experimental set-ups, statistical analyses and repeatability analyses are provided in the Appendix 2.

At the field station, individually marked mouse lemurs were housed in cages of $80 \mathrm{~cm} \times 80 \mathrm{~cm} \times 80 \mathrm{~cm}$ equipped with a nest box, several branches, an experimental platform, and ad libitum access to water. We kept animals for a maximum of three $(\mathrm{N}=488$; in 65 cases four, in 17 cases five) nights, after which they were released at dusk at their site of capture. In total, we tested up to 150 mouse lemurs per task in a total of 1,104 tests. Sample sizes differ between tasks as it was not possible to recapture all individuals until they have participated in all tasks of the test battery (Appendix 2: Table S2-1).

Testing started between 18:00 and 19:00h under red light conditions, when subjects were active and motivated, and ended when the motivation of the animals decreased. The experimental test battery comprised two personality tests, an open field test and a novel object test, and ten cognitive tests (food extraction task, persistence test, discrimination and reversal learning paradigms with visual discrimination, visual reversal learning, spatial discrimination, and spatial reversal learning, plus maze, cylinder test, two stringpulling tasks), for which we used small pieces of banana as food rewards (Appendix 2: Table S2-1; Fig. 3.1). 


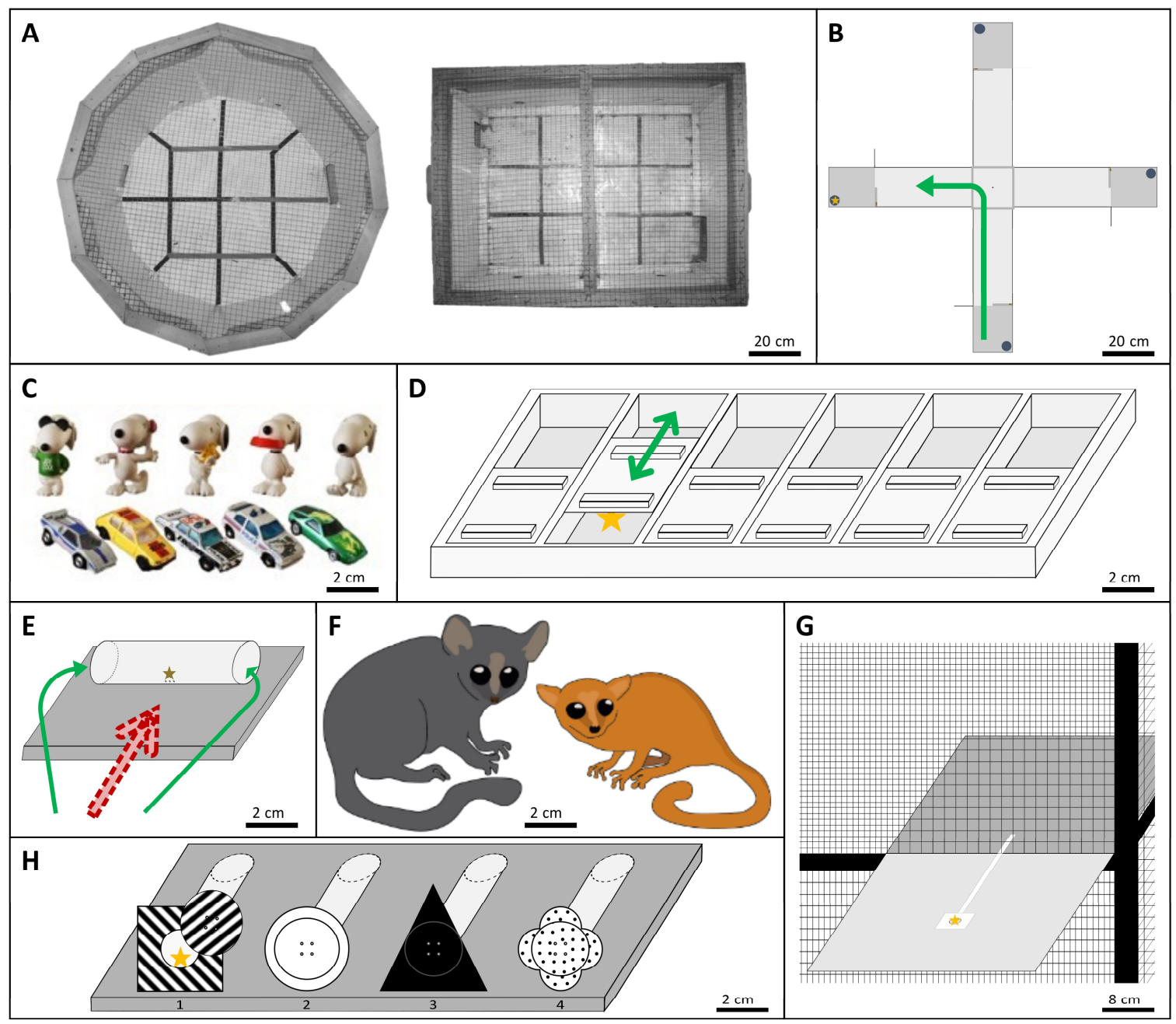

Fig. 3.1 Experimental test battery and mouse lemurs. A) Arenas used in the open field test. B) Plus maze. C) Objects used in the novel object test. D) Food extraction task and persistence test. E) Cylinder test. F) Left: gray mouse lemur, right: Madame Berthe's mouse lemur; scaled to size differences. G) String-pulling task, singlestring set-up. H) Apparatus used for the visual and spatial discrimination and reversal learning paradigm; numbers indicate the position of the forms. Green filled arrows indicate correct routes. Red striped arrows indicate incorrect routes. Yellow stars represent a food reward.

\subsubsection{Personality tests}

We assessed an individual's explorative tendencies in an unknown environment, using an open field test (Fig. 3.1A). After subjects entered the arena voluntarily, they were observed for $5 \mathrm{~min}$ exploring the arena. We used the duration the subjects spent locomoting as measure for exploration (Appendix 2: Table S2-2). To assess an individual's neophilic tendencies, we introduced a novel object (Fig. 3.1C) directly after each open field test into the arena. We counted the number of contacts over the course of a 5 min test duration and used this contact frequency as our measure for neophilia. 


\subsubsection{Food extraction task}

To assess innovative propensities, individuals could extract up to six food rewards from a problem-solving box with six uniform wells (Fig. 3.1D) within a test duration of $20 \mathrm{~min}$. As initial innovation speed, we measured the success latency as the time span between the response (i.e., entering the experimental platform and visualizing the task) and the first success (i.e., extracting the $1^{\text {st }}$ piece of banana; solver). In case an individual did not succeed at all (non-solver), we set its success latency to $20 \mathrm{~min}$ as the maximum test duration. Additionally, we counted the number of successes as measure for repeated innovative propensity.

\subsubsection{Persistence test}

To assess an individual's persistence in manipulating an object with potential access to food, we modified the problem-solving box, in that five of the six lids were blocked and only one of the six rewards could be extracted. We calculated an individual's persistence rate by dividing the duration manipulating the box by the duration being in contact with the box and noted whether an individual opened the well (solver) or not (non-solver).

\subsubsection{Visual and spatial discrimination and repeated reversal learning paradigm}

To assess an individual's associative and flexible learning abilities, we used a repeated discrimination and reversal learning paradigm with four separate tasks. On a plate, we positioned four tubes that only differed in shape and pattern of a form at the front part (Fig. 3.1H). Attached to the form was a lid that could be easily rotated to obtain access to the food reward in case of the $S^{+}$. For the first task, the visual discrimination, and the second task, the visual reversal, the shape and pattern of the form served as cue to locate the $S^{+}$. In the third task, the spatial discrimination, and the fourth task, the spatial reversal, the shape and pattern became irrelevant, and the position of the form served as $\mathrm{S}^{+}$. Across sessions of 15 trials, we counted the number of correct trials (i.e., manipulating only the $\mathrm{S}^{+}$ form and extracting the food reward) which we used as measure for associative learning 
abilities. After the subject had correctly chosen the $\mathrm{S}^{+}$-form for at least 24 out of 30 trials (80\%-learning criterion over two consecutive sessions), we proceeded with the next experimental task and used the total number of trials to reach this learning criterion as measure for the overall performance per task. As measure of flexibility, we calculated a transfer index (TI, Equation 1) for the transitions with changing reward contingencies (i.e., from the visual discrimination to the visual reversal, from the visual reversal to the spatial discrimination, and from the spatial discrimination to the spatial reversal).

$$
\text { Equation } 1 \quad \text { TI }=\frac{\text { post-reversal performance }}{\text { pre-reversal performance }}
$$

\subsubsection{Plus maze}

To assess an individual's spatial learning abilities and spatial memory, we set up a plus maze with four arms leading to four terminal boxes (Fig. 3.1B), of which only one was baited (goal box). We counted how often a subject entered the wrong arm and/or box per trial and summed it up to an error score per trial, which we defined as spatial learning performance. For the overall spatial performance, we used the mean sum of errors throughout one session of 15 trials.

\subsubsection{Cylinder test}

To assess an individual's inhibitory control, we conducted a detour-reaching task using the cylinder test design (MacLean et al. 2014) (Fig. 3.1E). After an initial training session with an opaque cylinder (see Appendix 2), we conducted the experimental session using a transparent cylinder. Throughout one session of ten trials, we counted the number of incorrect trials, i.e., when the subject did not take the detour as a first response to get access to the food reward in the center of the transparent cylinder. 


\subsubsection{String-pulling task, single-string set-up}

To assess an individual's means-end understanding, we conducted a string-pulling task in the single-string set-up (Fig. 3.1G). Within a 20 min test duration, the subject could pull a cable tie to access the food reward at the outer end of the cable tie. We measured the success latency as timespan between the response and reaching the reward. For subjects that did not succeed (non-solver), we set the success latency to the maximum time of the trial (20 min) plus the response latency. We used this success latency as proxy for meansend understanding.

\subsubsection{String-pulling task, perpendicular strings set-up}

To assess an individual's goal directedness, we conducted a string-pulling task with a perpendicular strings set-up, as modification of the single-string set-up, where a second, non-baited cable tie was presented. In a session of ten trials, we counted the number of incorrect trials, i.e., the subject did not succeed, or it manipulated the incorrect string, which we used as proxy for goal directedness.

\subsection{Statistical analyses}

\subsubsection{Variation in cognitive performances}

We conducted all statistical analyses in $\mathrm{R}$ (version 4.0.0, R Core Team, 2020), using multivariate (mixed) models to examine interspecific and intraspecific variation in performances (gaussian linear models (LM), gaussian linear mixed models (LMM), negative binomial models (NBM), negative binomial mixed models (NBMM), zeroinflated negative binomial models (0-infl NBM), cox-proportional hazards models (cox PHM), poisson models (PM)), and factor analytical approaches to examine general intelligence factors. Since there is no sexual dimorphism in either species, but body mass changes occur as a result of sex-specific energy strategies, as well as with hormonally induced somatic changes (Eberle and Kappeler 2002; Dammhahn and Kappeler 2005), we controlled for sex and body condition using the body mass index (BMI) as a proxy. In addition, we controlled for stable individual differences in behavior, i.e., personality traits, 
since an individual's exploration level or neophilic tendencies can potentially influence its engagement in experimental tasks and, subsequently, its performance level. In principle, we examined interspecific variation in performances by setting species, sex, and age (log-transformed) as fixed factors. To examine intraspecific variation in performance, we set sex, season, BMI (log-transformed) and personality factors as fixed factors. We tested for interactions of species and sex with other fixed factors but included the interaction only if the model significantly differed from the model without interactions. To test the significance of the predictors as a whole, we compared all full models with the respective null model comprising only the intercept and potential random factors (see Appendix 2; Forstmeier and Schielzeth 2011).

\subsubsection{General intelligence}

Finally, we investigated general intelligence across, as well as within species. For the interspecific G-factor, we calculated two principal axis factor analyses, using the function 'fa' with the argument 'fm' set to 'pa' ('psych' package). The first PAF contained performance scores of individuals that completed all tests ( $N=20 \mathrm{GML}, \mathrm{N}=9 \mathrm{MBML})$. For the second PAF, we used performance scores of individuals that completed all tests, except for the discrimination and reversal learning paradigm, resulting in a larger sample size ( $\mathrm{N}=76 \mathrm{GML}, \mathrm{N}=19 \mathrm{MBML}$ ). We controlled for sphericity by applying the Bartlett's test and for sampling adequacy by applying the KMO.

For the intraspecific $g$-factor, we used the same (log-transformed) performance scores as for the $G$-factor. For each species separately, we calculated two PCAs per species. The first PCA per species contained the performance scores of all tests excluding the spatial discrimination and the spatial reversal, which reduced the data sets to $N=21 \mathrm{GML}$ and $\mathrm{N}=9 \mathrm{MBML}$. For the second PCA per species, we excluded all performance scores of the repeated discrimination and reversal learning paradigm, achieving a sample size of $\mathrm{N}=76 \mathrm{GML}$ and $\mathrm{N}=19 \mathrm{MBML}$. For each PCA, we controlled for sphericity by applying the Bartlett's test and for sampling adequacy by applying the KMO. 


\section{Results}

\subsection{Interspecific comparisons}

\subsubsection{Personality: open field and novel object test}

Since locomotion loaded most strongly on the first principal component and was most repeatable (Appendix 2: Table S2-4), we retained this variable as personality trait exploration. Variation in exploration was predicted by an interaction between species and sex (LM: $p=0.018$; Appendix 2: Table S2-5, model: a). Female MBML were more explorative than males as well as GML (Fig. 3.2A). Age did not predict variation in exploration.

In the novel object test, approach speed and contact frequency were poorly repeatable (approach speed: ICC $=0.158$; contact frequency: ICC $=0.106$ ). Since approach speed was skewed towards individuals that did not contact the novel object, we retained contact frequency as a measure of neophilia. About one third of the individuals of both species $(\mathrm{N}=28$ out of $90 \mathrm{GML}$ and $\mathrm{N}=8$ out of $24 \mathrm{MBML})$ did not contact the novel object. The full model estimating variation in neophilia did not significantly differ from the null model (0-infl NBM: $p=0.073$, Appendix 2: Table S2-5, model: b). Thus, variation in neophilia was predicted by neither species $(p=0.489)$, sex $(p=0.791)$, nor age $(p=0.621)$.

\subsubsection{Food extraction task: problem solving}

Variation in innovation speed (latency to $1^{\text {st }}$ success) differed between species (cox PHM: $\mathrm{p}=0.013)$ and sexes $(\mathrm{p}=0.044$; Appendix 2: Table S2-6, model: a). GML were faster to extract the first food reward than MBML, and males of both species were faster than females. Age did not predict innovation speed $(p=0.209)$.

Variation in innovative propensity ( $\mathrm{N}$ opened wells) differed between species (Appendix 2: Table S2-6, model: b). GML opened more wells than MBML (PM: $p=0.006$, Fig. 3.2B). Sex $(p=0.670)$ and age $(p=0.874)$ did not predict innovative propensity in either species. Both measures were repeatable (innovation speed: ICC $=0.605$, innovative propensities: ICC=0.405; $\mathrm{N}=21$ individuals: $15 \mathrm{GML}$ and $6 \mathrm{MBML}$ ). 

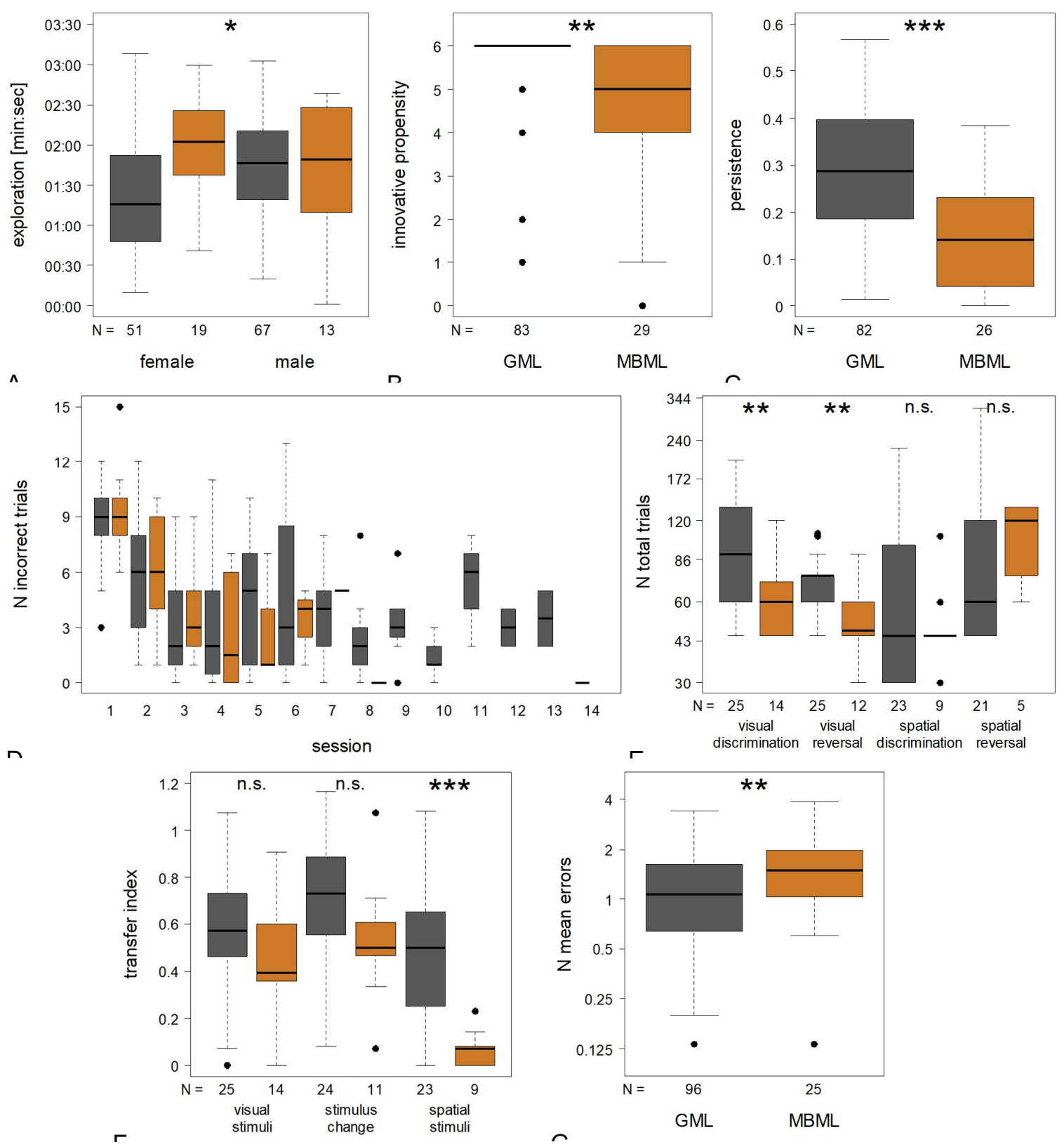

Fig. 3.2 A) Open field test: time spent exploring for females and males. B) Food extraction task: innovative propensity (N opened wells) per species. C) Persistence test: duration spent manipulating the box as a rate to the duration spent in contact with the box per species. D) Visual discrimination learning across sessions per species $(\mathrm{p}<0.05)$. E) Visual discrimination and reversal learning paradigm: overall performances within tasks. F) Visual discrimination and reversal learning paradigm: flexible learning between tasks. G) Plus maze: spatial memory performance per species. Sample sizes (N) are given below each boxplot. Gray: GML, orange: MBML. Significance levels: ${ }^{*} \mathrm{p}<0.05,{ }^{* *} \mathrm{p}<0.01,{ }^{* * *} \mathrm{p}<0.001$

\subsubsection{Persistence test}

Variation in persistence was predicted by species (LM: $\mathrm{p}<0.001$; Appendix 2: Table S2-7, model: a). GML were more persistent than MBML (Fig. 3.2C). Sex $(p=0.198)$ and age $(p=0.090)$ did not predict persistence in either species. Persistence was repeatable 
(ICC $=0.725, \mathrm{~N}=15$ individuals, $9 \mathrm{GML}$ and $6 \mathrm{MBML}$ ). Persistence across the two food extraction tests correlated positively (Spearman $r h o=0.382, \mathrm{p}<0.001, \mathrm{~N}=108$ ) and was repeatable $(\mathrm{ICC}=0.394)$.

\subsubsection{Visual discrimination}

Variation in visual discrimination learning was predicted by species in an interaction with session (NBMM: $p=0.020)$ and age $(p=0.015$; Appendix 2: Table S2-8, model: a). Both species decreased the number of incorrect trials across sessions, but this decrease was less pronounced in GML than in MBML (Fig. 3.2D). In both species, older individuals had more incorrect trials across sessions than younger individuals. Sex did not predict variation in visual discrimination learning across sessions $(p=0.102)$. Variation in the overall visual discrimination performance was predicted by species (LM: $\mathrm{p}=0.009$; Appendix 2: Table S2-8, model: b; Fig. 3.2E) and age ( $\mathrm{p}=0.008)$. GML and older individuals reached the learning criterion after more trials than MBML and younger individuals, respectively. Sex did not predict variation in visual discrimination performance $(\mathrm{p}=0.458)$.

\subsubsection{Visual reversal learning}

Variation in visual reversal learning was predicted by species (NBMM: $p=0.001$ ), session $(p<0.001)$ and age $(p=0.018$, Appendix 2: Table S2-9, model: a). Both species decreased the number of incorrect trials across sessions. However, this decrease was less pronounced in GML than in MBML. Older individuals had fewer incorrect trials across sessions than younger individuals. Sex did not predict variation in visual reversal learning across sessions $(\mathrm{p}=0.758)$. Variation in the overall visual reversal performance was predicted by species (LM: $\mathrm{p}=0.011$, Appendix 2: Table S2-9, model: b; Fig. 3.2E). GML reached the learning criterion after more trials than MBML $(p=0.003)$. Sex $(p=0.614)$ and age $(p=0.186)$ did not predict variation in the visual reversal performance.

Neither species (LM: $p=0.310$; Fig. 3.2F), sex $(p=0.192)$, nor age $(p=0.054$; Appendix 2: Table S2-9, model: c) had a significant effect on the transfer index (TI), i.e., flexible learning from visual discrimination to visual reversal learning. The TI did not 
correlate with the number of trials to reach the learning criterion in the visual reversal learning (Spearman rho $=0.006, \mathrm{p}=0.974, \mathrm{~N}=36$ ) or with the innovative propensity (Spearman rho $=0.144, \mathrm{p}=0.401, \mathrm{~N}=36$ ).

\subsubsection{Spatial discrimination}

Variation in spatial discrimination learning was predicted by species in an interaction with session (NBMM: $p=0.002)$, sex in an interaction with session $(p=0.001)$, age in an interaction with species $(p=0.005)$, and by the position of the rewarded tube $S^{+}(p<0.001$, Appendix 2: Table S2-10, model: a). Both species and sexes decreased the number of incorrect trials across sessions. However, in GML and females this decrease was less pronounced than in MBML and males, respectively. While older GML had more incorrect trials across sessions than younger individuals, older MBML had fewer incorrect trials than younger ones. Mouse lemurs that learned to associate position $1\left(\mathrm{~S}^{+}\right)$with the food reward had fewer incorrect trials than individuals learning the positions 2, 3 or 4 . Variation in spatial discrimination performance was predicted by species (Fig. 3.2E) in an interaction with age (LM: $p=0.043)$, and by the $S^{+}(p=0.002$, Appendix 2: Table S2-10, model: b). While older GML reached the learning criterion after more trials than younger individuals, the effect was reversed for MBML. Position 1 was learned after fewer trials than the other three positions. Sex $(p=0.931)$ did not predict variation in spatial discrimination performance.

The TI from visual reversal learning to spatial discrimination was predicted by sex (LM: $p=0.014$ ), with males achieving a higher TI than females, but not by species $(p=0.066$; Fig. 3.2F $)$ and age $(p=0.680 ;$ Appendix 2: Table S2-10, model: $c)$ The TI correlated negatively with the number of trials to reach learning criterion in the spatial discrimination task (Spearman rho $=-0.463, \mathrm{p}=0.008, \mathrm{~N}=32$ ). Hence, initially more flexible individuals (higher TI) were faster learners (fewer trials to reach the learning criterion). However, TI did not correlate with innovative propensity (Spearman rho $=0.039$, $\mathrm{p}=0.831, \mathrm{~N}=33$ ). 


\subsubsection{Spatial reversal learning}

Variation in spatial reversal learning was predicted by species in interaction with session (NBMM: $p=0.004)$, sex $(p=0.034)$, and by the $S^{+}(p=0.041$, Appendix 2: Table S2-11, model: a). In both species, the number of incorrect trials decreased across sessions. However, in GLM this decrease was less pronounced than in MBML. Females had fewer incorrect trials across sessions than males. Mouse lemurs that learned to associate position 1 with the food reward $(N=7)$, had fewer incorrect trials than others (position 2: $N=5$, position 3: $\mathrm{N}=6$, position 4: $\mathrm{N}=8$ ). Age did not predict variation in spatial reversal learning $(\mathrm{p}=0.931)$. Variation in the overall spatial reversal performance was predicted by sex in interaction with the $\mathrm{S}^{+}(\mathrm{LM}: \mathrm{p}=0.012$, Appendix 2: Table S2-11, model: b). Male mouse lemurs that learned to associate position 3 with the food reward $(\mathrm{N}=3)$, reached the learning criterion after more trials than the others. Species $(p=0.267 ;$ Fig. 3.2E) and age $(\mathrm{p}=0.122)$ did not predict variation in the spatial reversal performance.

The TI from spatial discrimination to spatial reversal learning was predicted by species (LM: p<0.001), with GML achieving a higher TI than MBML (Appendix 2: Table S2-11, model: c; Fig. 3.2F). Sex $(p=0.168)$ and age $(p=0.492)$ did not predict variation in flexible learning. TI correlated negatively with the number of trials to reach the learning criterion in the spatial reversal learning task (Spearman rho $=-0.522, \mathrm{p}=0.006, \mathrm{~N}=26$ ). Hence, initially more flexible individuals (higher TI) were faster learners (fewer trials to reach the learning criterion). However, TI did not correlate with innovative propensity (Spearman rho $=0.112, \mathrm{p}=0.549, \mathrm{~N}=31$ ). Since we only tested one to two GML repeatedly in 2-4 of the tasks, we could not estimate repeatability of the performance scores in this experiment.

\subsubsection{Plus maze: spatial memory}

Variation in spatial learning was predicted by species in an interaction with trial (NBMM: $\mathrm{p}=0.005)$ and by the goal box $(\mathrm{p}<0.001$; Appendix 2: Table S2-12, models: $a)$. Both species made fewer errors across trials, and mouse lemurs assigned to the straight goal box $(\mathrm{N}=22)$ made fewer errors across trials than those assigned to the left $(\mathrm{N}=52)$ or right 
goal box $(\mathrm{N}=47)$. Sex $(\mathrm{p}=0.254)$ and age $(\mathrm{p}=0.537)$ did not predict variation in spatial learning across trials.

Variation in spatial memory was predicted by species $(\mathrm{LM}: \mathrm{p}=0.003)$ and by the goal box ( $<<0.001$, Appendix 2: Table S2-12, model: b). GML made fewer errors than MBML (Fig. 3.2G). Mouse lemurs assigned to the left or right goal box made more errors than those assigned to the straight goal box. Sex $(p=0.361)$ and age $(p=0.429)$ did not predict variation in spatial memory. Spatial memory was repeatable ICC $=0.412$ ( $\mathrm{N}=21$ individuals, 15 GML and six MBML).

\subsubsection{Cylinder test: inhibitory control}

In the inhibitory control task, individuals that needed more trials to reach the learning criterion prior to testing made more errors during the testing session (NBM: $p=0.005$ ). Variation in inhibitory control was not predicted by either species $(\mathrm{p}=0.126)$, $\operatorname{sex}(\mathrm{p}=0.783)$, or age ( $\mathrm{p}=0.319 ;$ Appendix 2: Table S2-13, model: $b)$.

Inhibitory control did not correlate with innovative propensity (Spearman rho $=-0.152, \quad \mathrm{p}=0.121, \mathrm{~N}=105$ ), flexible learning (TI from visual reversal learning: Spearman rho $=-0.096, \mathrm{p}=0.576, \mathrm{~N}=36$; $\mathrm{TI}$ visual reversal to spatial discrimination learning: Spearman rho $=-0.030, \mathrm{p}=0.869, \mathrm{~N}=36$; TI from spatial reversal learning: Spearman rho $=-0.026, \mathrm{p}=0.891, \mathrm{~N}=33$ ) or overall learning performances (visual discrimination: Spearman rho $=-0.133, \mathrm{p}=0.439, \mathrm{~N}=36$; visual reversal: Spearman rho $=-0.007, \mathrm{p}=0.970, \mathrm{~N}=34$; spatial discrimination: Spearman rho $=0.008, \mathrm{p}=0.966$, $\mathrm{N}=31$; spatial reversal: Spearman rho $=0.078, \mathrm{p}=0.713, \mathrm{~N}=25$ ). The number of training trials ( $\mathrm{ICC}=0.556$ ) were repeatable, but the number incorrect test trials were only poorly repeatable $(\mathrm{ICC}=0.141)$.

\subsubsection{String pulling task: means-end understanding and goal directedness}

Performance in the means-end understanding (cox PHM: $\mathrm{p}=0.600$ ) and goal directedness (PM: $\mathrm{p}=0.874)$ was not predicted by any of the investigated factors (Appendix 2: Table S2-14, 
model: $\mathrm{a}, \mathrm{b}$ ). We estimated the repeatability for $\mathrm{N}=11$ individuals (7 GML and 4 MBML) that repeated this test (response latency: ICC=-0.137, success latency: ICC=-0.192).

\subsection{General intelligence}

We did not find evidence for an interspecific $G$-factor in either mouse lemur species. The performance scores did not load similarly onto the first component of the PAF, including data from the larger sample size (including tests on problem solving, spatial memory, means-end understanding, goal directedness, inhibitory control), and Bartlett's tests of sphericity was non-significant, indicating a generally low correlation across performance scores (Appendix 2: Table S2-15). The results were similar for the reduced PAF (including tests on visual and spatial discrimination as well as reversal learning, Appendix 2: Table S2-16).

\subsection{Intra-specific variation in performance in personality and cognitive tests}

We also investigated whether intra-specific performance in these tests was influenced by sex, age, body mass index, or in case of cognitive tests, also by variation in personality traits. In both species, variation in performance scores was only occasionally explained by these individual characteristics, with no systematic variation within species. We also did not find evidence for an intraspecific $g$-factor. The complete results and the discussion thereof can be found in the Appendix 2 (GML: Table S2-17, MBML: Table S2-18).

\section{Discussion}

Gray and Madame Berthe's mouse lemurs differed in 13 of 21 performance measures (Table 3.1), which were moderately repeatable in the majority of tests and, hence, consistent within individuals (Appendix 2: Table S2-2). Overall, the ecological generalist GML were more innovative, more persistent, showed better spatial learning and memory, and greater flexibility when spatial stimulus-reward contingencies were changed. The 
ecologically more specialized MBML were more explorative and learned visual and spatial reward contingencies faster, achieving better total performances in visual association learning. However, the two species did not differ in neophilia, inhibitory control, means-end understanding or goal directedness. Moreover, we did not find evidence for an interspecific G- or intraspecific $g$-factor. In summary, our study provides support for domain-specific cognitive co-evolution with ecological factors, and a particular advantage of generalists in confronting novel challenges with a greater innovative potential and greater flexibility when confronted with changing spatial stimuli.

Table 3.1 Overview of species differences in performance across tasks. ( $)$ ) indicates better and ( $)$ indicates worse performance, whereas (-) indicates no difference in performance.

\begin{tabular}{|c|c|c|c|c|}
\hline Task & GML & MBML & Age & Sex \\
\hline Activity & - & - & - & 凤 GML: females \\
\hline Neophilia & - & - & - & $\sqrt{n}$ males \\
\hline \multicolumn{5}{|l|}{ Innovation: } \\
\hline speed & 仓 & $\sqrt{ }$ & - & 仓 males \\
\hline propensity & $\hat{v}$ & $\sqrt{2}$ & - & - \\
\hline persistence & $\hat{\varphi}$ & $\sqrt{n}$ & - & - \\
\hline \multicolumn{5}{|l|}{ Visual discrimination: } \\
\hline learning & $\sqrt{3}$ & 仓 & $\sqrt{ }$ old & - \\
\hline performance & I & 仓 & $\sqrt{ }$ old & - \\
\hline \multicolumn{5}{|l|}{ Visual reversal: } \\
\hline learning & $\sqrt{2}$ & 仓 & 仓े old & - \\
\hline performance & 约 & 仓 & - & - \\
\hline \multicolumn{5}{|l|}{ Spatial discrimination: } \\
\hline learning & $\sqrt{3}$ & 仓 & 凤 old GML, 仓 old MBML & 仓े males \\
\hline performance & - & - & 凤 old GM,L 仓 old MBML & - \\
\hline \multicolumn{5}{|l|}{ Spatial reversal: } \\
\hline learning & 』 & 仓 & - & $\S$ males \\
\hline performance & - & - & - & $\sqrt{3}$ males: position 3 \\
\hline \multicolumn{5}{|l|}{ Flexibility (TI) } \\
\hline visual & - & - & - & - \\
\hline visual - spatial & - & - & - & 仓े males \\
\hline spatial & 仓 & $\sqrt{2}$ & - & - \\
\hline \multicolumn{5}{|l|}{ Spatial memory: } \\
\hline learning & 仓े & $\sqrt{ }$ & - & - \\
\hline performance & 仓े & $\sqrt{3}$ & - & - \\
\hline Inhibitory control & - & - & - & - \\
\hline Means-end understanding & - & - & - & - \\
\hline Goal directedness & - & - & - & - \\
\hline
\end{tabular}


In both species, performance in personality and cognitive tests was weakly and inconsistently influenced by individual characteristics and moderately repeatable, reflecting general patterns on intra-individual consistency in cognitive performances in animals (Cauchoix et al. 2018). Regarding personality traits, both species did not differ in exploration. However, female MBML were more explorative than males in comparison to GML. In addition, the two species did not differ in their neophilic response, supporting neither the Dangerous-Niche Hypothesis (i.e., generalists should be more neophobic as they may encounter more dangerous situations) nor the Neophobia Threshold Hypothesis (i.e., generalists should be less neophobic as they may have more diverse prior experiences; Greenberg 1983; Greenberg 2003).

Concerning cognitive performance, GML were indeed more innovative and achieved higher flexibility scores, at least under changing spatial stimuli. These abilities may allow them to respond more adaptively to dynamically changing habitats and anthropogenic influences (Schäffler and Kappeler 2014; Rakotoniaina et al. 2016). In our study region, the abundance of GML is generally higher than that of MBML, especially in habitats with anthropogenic influence, such as edge habitats (Schäffler and Kappeler 2014; Rakotoniaina et al. 2016). Similarly, in Northern Madagascar, GML were largely unaffected by habitat fragmentation, while the abundance of the sympatric but ecologically more specialized golden-brown mouse lemurs (M. ravelobensis) decreased with increasing habitat fragmentation (Andriatsitohaina et al. 2020). Increasing anthropogenic activities, such as deforestation or habitat fragmentation contribute to an alarming species loss in Madagascar (Schwitzer et al. 2014a). Such environmental changes may eventually promote a species turnover towards ecological generalists, whereas more specialized species may suffer from decreased population size (Irwin et al. 2010; Markolf et al. 2020). Our study therefore indicates potential cognitive constraints of ecological specialization that may help to explain why some species experience a higher extinction risk in the face of ongoing environmental changes.

MBML learned the associative reward contingencies faster; a characteristic that allows specialists to experience advantages over generalists in stable environmental conditions (Wilson and Yoshimura 2008). Specifically, MBML's better motor control to abandon previously successful behaviors (Henke-von der Malsburg and Fichtel 2018) and 
their lower persistence to produce a behavior that does not lead to success (this study), may have contributed to their superior performance, at least in visual associative learning experiments. GML feed relatively more often on tree gum, a foraging strategy associated with enhanced inhibitory control (Stevens et al. 2005). However, the two species did not differ in inhibitory control, suggesting that the fact that they feed on gum rather than the relative frequency of this foraging behavior is associated with superior inhibitory control. Both inhibitory control and reversal learning abilities reflect behavioral flexibility (MacLean et al. 2014; Beran 2015). However, these measures did not correlate with each other, suggesting that they reflect different aspects of cognitive flexibility in mouse lemurs. The TI is a standard proxy for flexible learning abilities, reflecting the potential to switch between tactics when conditions change, i.e., in the context of a first response to a modified reward contingency (Rumbaugh et al. 1972). However, it does not indicate how well the old tactic will be abandoned in favor of the new tactic. This might be better reflected by the number of trials until criterion after reversal or the number of perseverative errors across sessions, because these measures better reflect how quickly individuals may overcome the previously learned reward contingency and, therefore, how flexible an animal switches between strategies (Hauser 1999). The TI and the number of trials to reach the criterion after reversal correlated negatively with each other in the transfer from the visual to spatial stimuli and in the spatial reversal learning, indicating that individuals that responded more flexibly to the reversed reward contingency learned this reward contingency faster in spatial learning. Hence, with regard to spatial stimuli, which might be ecologically more relevant than abstract forms as in the visual reversal paradigm, mouse lemurs were able to switch flexibly between strategies, and GML were more flexible when confronted with changing spatial stimuli than MBML.

The plus maze assays spatial learning and memory that is essential for effective spatial navigation. GML learned this task faster than MBML, supporting earlier results on their spatial learning and high travel efficiency (Lührs et al. 2009). Although MBML have larger home ranges and should therefore have better spatial abilities, the ability to adapt to different habitat types may require more flexible spatial learning abilities and may therefore better explain from an evolutionary perspective why GML performed better in 
this task and also why they responded more flexibly when spatial stimulus-reward contingencies changed.

In tasks with little ecological relevance, where species differences in cognitive performance were not predicted regarding the degree of ecological specialization, such as inhibitory control, means-end understanding or goal directedness, both species performed on par, suggesting that they did not differ per se in cognitive performance. These results also support our interpretation of the adaptive nature of the observed differences as being driven by different ecological factors.

In humans, cognition has evolved towards positively correlating generally highlevel cognitive abilities (Herrmann et al. 2007; Burkart et al. 2017). However, a general correlation between cognitive abilities and brain size has received mixed support in comparative studies of animals (Burkart et al. 2017). In mouse lemurs, we did not find evidence for general intelligence, neither on the interspecific, nor on the intraspecific level. Our results rather support the Domain-Specific Hypothesis (Cosmides and Tooby 1994), which postulates enhanced abilities in only some cognitive domains, whereas abilities in others remain on more basic performance levels (Poirier et al. 2020).

Finally, this study raises new questions about the evolutionary mechanisms driving cognitive adaptations to environmental features. From a phylogenetic perspective, the split between the basic lineages to which these two species belong occurred about 810 million years ago. Phylogenetic reconstructions of the speciation patterns in mouse lemurs suggests that the longitudinal dispersal along the west coast of Madagascar by GML was achieved with relative ease throughout the Pleistocene (Yoder et al. 2016). However, habitat fragmentation via Holocene droughts may have erected natural barriers such as rivers, creating several centers of endemism (Wilmé et al. 2006), isolating some species, such as MBML, in small ranges. Thus, gray mouse lemurs actually had more time available to evolve cognitive and ecological adaptations to the habitat in which they now co-occur, but they appear to have retained cognitive abilities that may provide ecological advantages across their entire range. Genetic studies investigating patterns of gene flow and heritability in different cognitive abilities are now indicated to begin exploring the evolutionary mechanisms shaping the links between ecological adaptations and cognitive constraints. 


\section{Conclusions}

We show that direct comparisons of cognitive performances between sympatric sisterspecies with a similar social system can help to unfold the role of ecological factors in the evolution of cognition. Species-specific ecological adaptations covary with cognitive abilities. The ecologically more generalist species was particularly more innovative, persistent, exhibited better spatial learning abilities, spatial memory, as well as spatial flexibility than the specialist, affording them with the behavioral flexibility to respond adaptively to rapidly changing habitats and anthropogenic disturbances. How these differences in cognitive abilities have been maintained over millions of generations in local sympatry requires further study.

\section{Acknowledgments}

This research was funded by the Deutsche Forschungsgemeinschaft (DFG), awarded to PMK (grant number KA 1082/29-565 2) and CF (grant number FI 929/10-1). We are grateful to Bruno Tsiverymana and the other members of the Equipe Kirindy for their help with capturing and housing mouse lemurs, and to Jan Förster, Joe Buchner, Luca Hahn, Svenja Blödorn, Joana Niedner, Colleen Illing, Annkatrin Pahl, Frederike Hoppmann, and Tímea Kovács for their help with collecting data and analyzing videos. We thank the Ministry of the Environment, the Mention Zoologie et Biodiversité Animale Université d'Antananarivo and the CNFEREF Morondava for their authorization and support of this study, Katja Rudolph for providing mouse lemur icons, and two referees for very constructive comments on an earlier version of this manuscript.

\section{Ethical approval}

This study was conducted in accordance with the German and Malagasy (Commission Tripartite CAFF) legal and ethical requirements of appropriate animal procedures. Research protocols and experimental procedures were approved by the Bundesamt für Naturschutz (BfN) in Germany and by the Ministère de l'Environnement et des Eaux et Forêts in Madagascar (MINEEF). Research in Kirindy was authorized by the Centre National de Formation, d'Etudes et de Recherche en Environnement et Foresterie (CNFEREF) Morondava. 


\section{Appendix 2}

This appendix contains more detailed information on:

Predictions... .77

Supplementary methods 78

Supplementary results and discussion. .97

Supplementary tables 100

\section{Predictions}

Regarding variation in personality, we predicted that Madame Berthe's mouse lemurs (MBML), which have larger home ranges, are more explorative than gray mouse lemurs (GML). In line with the Dangerous-Niche Hypothesis, high neophobia may protect individuals from potentially dangerous situations(Greenberg 2003). As GML may encounter more dangerous situations, we predicted them to be more neophobic than MBML. Alternatively, as the ecological generalist encounters a greater variety of habitat and/or food types, it should have more diverse prior experiences against which it can rate novelty (Neophobia Threshold Hypothesis(Greenberg 1983)). Accordingly, the GML should be less neophobic than MBML.

Regarding variation in cognitive abilities, we predicted that the generalist GML exhibit greater innovative potential and greater flexible learning abilities than the ecologically more specialized sister-species. Since MBML live in less variable environments, we predicted that they learn associated reward contingencies faster. We also predicted that they have better spatial memory because they feed preferentially on spatially clumped resources, whereas GML feed on more abundant and mobile food items(Dammhahn and Kappeler 2008a). Moreover, because MBML were more flexible in solving a more complex food extraction task, as they had better inhibitory motor control than GML(Henke-von der Malsburg and Fichtel 2018), we predicted GML to be more persistent. Self-control is generally associated with dietary breadth across 36 primates(MacLean et al. 2014), suggesting that dietary generalists should have better selfcontrol than dietary specialists. Since both species do not feed on dietary items requiring specific extractive foraging techniques, we did not predict significant interspecific 
differences regarding means-end understanding or goal directedness. We nonetheless included these tests to also examine cognitive performances in tasks without obvious ecological relevance(Henke-von der Malsburg et al. 2020), in which differences in

performance are not predicted by the degree of ecological specialization. Finally, given the contrasting predictions across the various cognitive abilities, we did not expect variation in performance across tasks to be correlated and to load onto one common general intelligence (G/g-)factor(Humphreys 1979; Deaner et al. 2006). Rather, we expected both species to have evolved domain-specific cognitive abilities (Domain-Specific Hypothesis(Cosmides and Tooby 1994)).

\section{Supplemental methods}

\section{Study animals: Capture and housing}

We baited Sherman live traps with a slice of banana, set them at dusk at trail intersections and collected them at dawn. Captured mouse lemurs were brought to the near-by field station and individually identified. All individuals were weighted. If they were newly captured, they were briefly restrained with $0.6 \mu \mathrm{l}$ ketamine $(50 \mathrm{mg} / \mathrm{ml})$ per $1 \mathrm{~g}$ body mass to mark them individually with a subdermal microtransponder (Trovan, Usling, Germany). If they were captured for the first time, we also took several standard morphologic measures(Eberle and Kappeler 2002). We calculated an individual's current body mass index (BMI) by dividing its bizygomatic head breadth [mm] by the individual's current body mass [g]. We estimated an individual's age by determining the number of days between birth and the date of the respective experimental test. We set an individual's birth date to the modal birth date 1 January of the year of its first capture(Eberle and Kappeler 2002). The individuals' ages ranged from 0.26 to 8.58 yrs. $($ mean $=1.21 \pm 1.40)$ in GML and from 0.33 to 5.35 yrs. (mean = $1.20 \pm 1.17$ ) in MBML.

At the field station, mouse lemurs were housed in cages of $80 \mathrm{~cm} \times 80 \mathrm{~cm} \times 80 \mathrm{~cm}$ equipped with a nest box, several branches, an experimental platform, and ad libitum access to water. We kept animals for a maximum of three $(N=488$; in 65 cases four, in 17 cases five) nights, after which they were released at dusk at their site of capture. Since the complete experimental test battery was usually not completed during one test session 
comprising three nights, we selectively recaptured subjects after they had spent at least three nights in the forest. The animals usually exhibited great trap happiness, with some individuals entering traps more than 100 times(Eberle and Kappeler 2002). Testing started each evening between 0600 and 0700 pm under red light conditions, which wavelengths is not visible for the dichromatic mouse lemurs (Peichl et al. 2019), and ended when the motivation of the animals decreased, usually around midnight. When a mouse lemur voluntarily left its nest box, the nest box was closed to facilitate later testing. Throughout the night, it could hide between branches and rest at any time. The mouse lemurs were fed after testing with cockroaches, moths and pieces of banana. In total, we tested up to 150 mouse lemurs per test in a total of 1,104 tests (Table S2-1).

\section{General experimental procedure}

The experimental test battery comprised two personality tests, an open field test and a novel object test, and ten cognitive tests (food extraction task, persistence test, discrimination and reversal learning paradigms with visual discrimination, visual reversal learning, spatial discrimination, and spatial reversal learning, plus maze, cylinder test, two string-pulling tasks; Table S2-1; Fig. 3.1). The order of the tests was randomized and counterbalanced between subjects. All subjects were naïve to experimental conditions, unless otherwise stated $(N=20$; Table S2-1). Subjects were captured on average $2.39 \pm 1.68$ times. To complete the relatively time-consuming discrimination and reversal learning paradigm, subjects were tested on average in $3.00 \pm 1.26$ test sessions (i.e., time periods of three nights), resulting in smaller sample sizes in these tests (Table S2-1). The remaining tests could be completed on average in $2.07 \pm 0.80$ test sessions.

We started with the experiments only when subjects were active and motivated, i.e., when they were actively moving around and could be easily lured with a stick covered with banana to the start position at the opposite end of the experimental platform. We used small pieces of banana as food rewards. Before any experimental session, we cleaned the experimental platform, the test apparatus, or the respective arena for the open field test and plus maze with $70 \%$-ethanol to remove any odor cues. In experiments comprising 
several trials, we repeated the cleaning procedure throughout the trials as stated in the respective test section.

Each experimental test session was videotaped (Sony HDR-CX 240) and later analyzed using BORIS(Friard and Gamba 2016). For each test, $10 \%$ of the videos were double-coded by a second observer naïve to the research question, resulting in a mean interobserver reliability of $96.0 \%$ (min.: $80.2 \%$, max.: 100\%; Table S2-2).

\section{General statistical analyses}

We conducted all statistical analyses in R (version 4.0.0, R Core Team, 2020), using multivariate (mixed) models to examine interspecific and intraspecific variation in performances (gaussian linear models (LM), gaussian linear mixed models (LMM), negative binomial models (NBM), negative binomial mixed models (NBMM), zeroinflated negative binomial models (0-infl NBM), cox-proportional hazards models (cox PHM), poisson models (PM)), and factor analytical approaches to examine general intelligence factors. Since there is no general sexual dimorphism in either species, but body mass changes occur as a result of sex-specific energetic strategies, as well as with hormonally induced somatic changes(Eberle and Kappeler 2002; Dammhahn and Kappeler 2005), we controlled for sex and body condition using BMI as a proxy. In addition, we controlled for stable individual differences in behavior, i.e., personality traits, since an individual's exploration level or neophilic tendencies can potentially influence its engagement in experimental tasks and, subsequently, its performance level.

We examined interspecific variation in performances by setting species, sex, and age (log-transformed) as fixed factors. To examine intraspecific variation in performance, we set sex, season, BMI (log-transformed) and personality factors as fixed factors. For models including data of a smaller sample size, we calculated several reduced models by retaining only a maximum of $N=10$ data points for fixed factors each. We scaled covariates to a mean of zero and a standard deviation of one to obtain comparable estimates and to achieve more easily interpretable coefficients. If a continuous variable was not symmetrically distributed (visual inspection), we log-transformed it before scaling. 
We tested for interactions of species and sex with other fixed factors but included the interaction only if the model significantly differed from the model without interactions. We first tested the overall effect of the fixed factors by comparing the selected full model's deviance with that of its null model comprising only the intercepts (and random factors, if applicable). These tests of significance were based on an analysis of variance (ANOVA) with F-test for gaussian linear models, Wald-test for cox-proportional hazards models, or likelihood ratio test (LRT) for other models. For the individual predictors, we derived respective test-statistics and p-values using the function 'drop1', which compares the deviance of the full model with that of the reduced model, lacking the respective individual predictor. For all tests, we used a significance level of 0.05 . We extracted sample size adjusted effect sizes $\left(\mathrm{R}^{2}\right)$ directly from the summary output for linear models. For other models, we calculated Nagelkerke's $R^{2}$. We checked various diagnostics of model validity and stability (Cook's distance using the function 'cooks.distance'; DFBetas using the function 'dfbeta'; DFFits using the function 'dffits'; overdispersion; variance inflation factors using the function 'vif' ('car' package(Fox and Weisberg 2011)) applied to standard linear models without any random factors; normality and homogeneity of residuals by plotting a qq-plot for the residuals and a scatterplot between residuals and fitted values), which never indicated obvious influential cases, nor obvious deviations from the respective model assumptions.

Additionally, we tested the repeatability of the measured performances, as well as the interobserver reliability of video codes using the intraclass correlation coefficient (ICC; 'ICCest' function, 'ICC' package(Wolak 2015)).

\section{Task-specific experimental procedure and statistical analyses \\ Open field test: Experimental set up}

We assessed an individual's explorative tendencies in an unknown environment, using either a rectangular $(80 \mathrm{~cm} \times 60 \mathrm{~cm} \times 60 \mathrm{~cm})$ or cylindric wooden arena $(\varnothing 80 \mathrm{~cm} \times 80 \mathrm{~cm}$; Fig. 3.1A). To avoid potential influences on the personality measures arising from initial neophobic responses due to the new housing conditions, we conducted this test only after subjects got used to the new housing condition, i.e., after the first night being in camp. 
After subjects entered the arena voluntarily, they were observed for $5 \mathrm{~min}$ exploring the arena. To measure the repeatability of personality traits, we subjected individuals repeatedly to the open field test, using the two arenas alternately. The first repetition was usually conducted when subjects were captured for the second time (number of days between tests: mean $=81.38 \pm 88.02$ ). Further repetitions were conducted each time when we re-captured the subject until it had accomplished the complete test battery, but for a maximum of two times per field season (days between test 2 and 3: mean $=130.14 \pm 122.12$; days between test 3 and 4 : mean $=66.14 \pm 76.32$ ). We tested 118 GML and 32 MBML at least once and one third of all individuals up to three times (Table S2-3). Only a small subset was tested in a fourth $(N=29)$, fifth $(N=8)$, sixth $(N=4)$ or seventh $(N=1)$ open field test. $N=16$ individuals were tested in the rectangular arena in previous studies conducted $11-47$ months before (mean $=25.12 \pm 15.75$; Table S21(Huebner et al. 2018; Henke-von der Malsburg and Fichtel 2018)). Based on videorecordings, we measured time spent locomoting and exploratory tendencies of subjects (Table S2-2).

\section{Open field test: Statistical analyses}

We visually inspected whether the variables of the first open field test were symmetrically distributed and applied a log-transformation to achieve a more symmetric distribution if necessary. We checked the correlation matrix of symmetrically distributed (log-transformed) variables using the function 'rcorr' ('Hmisc' package(Harrell 2021)) and controlled for sphericity by applying Bartlett's test (function 'cortest.bartlett', 'psych' package(Revelle 2020)), which was significant $\left(X^{2}=203.51, d f=10, p<0.001\right)$. Then, we conducted a principal component analysis (PCA) using the 'pca' function ('psych' package) with the standard varimax rotation. The Kaiser-Meyer-Olkin index (KMO; 'KMO' function, 'psych' package(Morton and Altschul 2019)) revealed good sampling adequacy with a value of 0.56 . We retained two components as suggested by the function 'fa.parallel' ('psych' package). The first component explained an individual's active exploration, with locomotion and cells traversed loading positively and the latency enter (log-transformed) loading negatively. The second component explained an individual's 
more passive, stationary exploration, with rears, i.e., standing on hindlegs, (log-transformed) and dips, i.e., inserting head into blind holes or closed doors of the open field, (log-transformed) loading positively (Table S2-4).

To estimate variation in exploration, we used a linear model with gaussian error distribution (' $1 \mathrm{~m}$ ' function) with the duration subjects spent locomoting as response variable. In the interspecific model (Table S2-5, model: a), we used species in interaction with sex, and age (log-transformed) as fixed factors. In the intraspecific models (Table S217, Table S2-18, models: a), we used sex, season, and BMI (log-transformed) as fixed factors.

\section{Novel object test: Experimental set-up}

To assess an individual's neophilic tendencies, we introduced a novel object (either a plastic snoopy or a metallic toy car; Fig. 3.1C) directly after each open field test into the arena. Out of the 150 individuals, only one was tested with a similar novel object in a previous study 11 months earlier (Table S2-1; (Henke-von der Malsburg and Fichtel 2018).)

We measured an individual's latency to approach the novel object by one grid cell (approach latency), to contact the novel object (contact latency), and the number of contacts over the course of a $5 \mathrm{~min}$ test duration (contact frequency). With the two latencies, we calculated the approach speed by subtracting the contact latency from the approach latency. If an individual did not contact the novel object, we set the approach speed to the sum of contact latency (which was set to the 5 min test duration) and the respective approach latency (which was also set to $5 \mathrm{~min}$ in case the individual did not approach the object). We chose the contact frequency as our measure for neophilia, as the other variables were highly skewed towards individuals that did not contact the object.

\section{Novel object test: Statistical analyses}

To estimate variation in neophilia, we used a zero-inflated negative binomial model with logit-link error function (function 'zeroinfl' with the argument 'dist' set to 'negbin'; 'pscl' package(Jackman et al. 2020)) and the number of contacts with the novel object as response 
variable. In the interspecific model (Table S2-5, model: b), we used species, sex, and age (log-transformed) as fixed factors in the count part and set sex as fixed factor in the zero part. In the intraspecific models (Table S2-17, Table S2-18, models: b), we used sex, season, and BMI (log-transformed) as fixed factors in the count part and set sex as fixed factor in the zero part.

\section{Food extraction task: Experimental set-up}

To assess an individual's innovative propensities, we presented a problem-solving box $(6 \mathrm{~cm} \times 12 \mathrm{~cm}$; Fig. 3.1D). Each of the six uniform wells $(5 \mathrm{~cm} \times 4.5 \mathrm{~cm})$ was baited with a small piece of banana, which could be extracted by sliding a lid open. After the box was introduced onto the experimental platform within the subject's cage, the animals had 20 min to extract the six rewards. We tested a total of $N=112$ individuals (83 GML and $29 \mathrm{MBML}) . \quad N=5$ individuals were subjected to this task in a previous study $11-53$ months earlier (mean $=34.30 \pm 15.31$; (Huebner et al. 2018)).

We measured an individual's response latency from the start of the test, when the box was deposited on the experimental platform, to its approach to the experimental platform, visualizing the task. As initial innovation speed, we measured the success latency as time span between the response latency and the first success (i.e., extracting the $1^{\text {st }}$ piece of banana; solver). In case an individual did not succeed at all (non-solver), we set its success latency to $20 \mathrm{~min}$ as the maximum test duration. Additionally, we counted the number of successes as measure for repeated innovative propensity. We estimated the repeatability of the variables by calculating the ICC for $N=21$ individuals (15 GML and six MBML), that repeated this test (response latency: $\mathrm{ICC}=-0.050$, success latency: ICC $=0.605$, success number: ICC $=0.405)$.

\section{Food extraction task: Statistical analyses, innovation speed}

To investigate interspecific variation in innovation speed (Table S2-6, model: a), we calculated a cox-proportional-hazards model (function 'coxph', 'survival' package(Therneau et al. 2020)) with the function Surv(success latency (log- 
transformed) I solver) as proportional hazard and species, sex, and age (log-transformed) as fixed factors. In the model for GML (Table S2-17, model: c), we used sex, season, BMI (log-transformed), activity, and neophilia as fixed factors. For MBML, we calculated four less complex models (Table S2-18, model: c) according to the small sample size. We set sex and season, sex, and BMI (log-transformed), sex in interaction with activity, and sex and neophilia as fixed factors, respectively.

\section{Food extraction task: Statistical analyses, innovation propensity}

To investigate interspecific variation in innovative propensity (Table S2-6, model: b), we calculated a poisson model with log link error function (function 'glm', family = 'poisson') with the number of successes as response and species, sex, and age (log-transformed) as fixed factors. In the intraspecific model for GML (Table S2-17, model: d), we used sex, season, BMI (log-transformed), activity, and neophilia as fixed factors. For MBML, we calculated four less complex models (Table S2-18, model: d) adapted to the low sample size in this species. We set sex and season, sex and BMI (log-transformed), sex and activity, and sex and neophilia as fixed factors, respectively.

\section{Persistence test: Experimental set-up}

To assess an individual's persistence in manipulating an object with potential access to food, we modified the problem-solving box, in that five of the six lids were blocked and only one of the six rewards could be extracted. Therefore, the persistence test always followed the food extraction test. If an individual did not open all the six wells during the food extraction test, we performed an additional training session prior to the persistence test $(N=17)$. This training session was similar to the food extraction test, with the only modification that all lids were opened by $5 \mathrm{~mm}$.

The persistence test lasted $20 \mathrm{~min}$ and was extended by $10 \mathrm{~min}$ in case the individual was still manipulating the box after $20 \mathrm{~min}(N=6)$. We calculated an individual's persistence as the duration manipulating the box in relation to the duration being in contact with the box and noted whether an individual opened the well (solver) or 
not (non-solver). We tested a total of $N=108$ (82 GML and $26 \mathrm{MBML})$. We estimated the repeatability of the variables by calculating the ICC for $N=15$ individuals (nine GML and six MBML), that repeated this test (manipulation: ICC $=0.570$, contact: $\mathrm{ICC}=-0.147$, persistence: ICC $=0.725)$. Additionally, we estimated the repeatability of persistence across tasks by calculating the ICC across the food extraction task and the persistence test for $N=108$ individuals (82 GML and $26 \mathrm{MBML})$.

\section{Persistence test: Statistical analyses}

To investigate variation in persistence, we calculated a linear model with gaussian error distribution. We used persistence as response variable and species, sex, and age (log-transformed) as fixed factors in the interspecific model (Table S2-7, model: a). Running the same model excluding the $N=17$ individuals that received additional training, did not affect the results (Table S2-7, model: b). In the intraspecific model for GML (Table S2-17, model: e), we used sex, season, BMI (log-transformed), activity, and neophilia as fixed factors. For MBML, we calculated four less complex models (Table S218, model: e) due to the low sample size for this species. We set sex and season, sex in interaction with BMI (log-transformed), sex and activity, and sex and neophilia as fixed factors, respectively.

\section{Visual and spatial discrimination and repeated reversal learning paradigm: Experimental set-up}

To assess an individual's associative and flexible learning abilities, we used a repeated discrimination and reversal learning paradigm with four individual tasks. On a plate, we positioned four tubes that only differed in shape and pattern at the front part (Fig. 3.1H). Tubes were fixed with clips on the plate, so that we could easily exchange the position between trials. The tube opening was surrounded of a plate in the form of either a square (pattern: black-white striped), a circle (pattern: white), a triangle (pattern: black), or flower (pattern: white with black dots). In front of each plate, we attached a lid that could be easily rotated to access a small piece of banana. As an odor control for non-rewarded 
tubes, we placed a piece of banana in the center of each tube, which, however, was not accessible to the subjects.

Before the first testing session, we conducted a familiarization session. In this session, the order of the tubes was always the same across the subjects (square - circle - triangle - flower) and each tube opening was baited with a food reward. We removed the lids, so that the animals could easily access the food reward in each of the tubes. A trial started when the apparatus was fully introduced on the experimental platform within the subject's cage and lasted a maximum of $10 \mathrm{~min}$, or until the subject retrieved all four food rewards. After the subject had retrieved all four food rewards in six familiarization trials, we started with the actual experiment.

In the first experimental task, the visual discrimination, we randomly selected one of the four forms serving as $\mathrm{S}^{+}$, containing the food reward. Across sessions of 15 trials, we randomized the position of the $\mathrm{S}^{+}$-form across the four positions in a counterbalanced order between the left (position 1 and 2) and right side (position 3 and 4 ) of the plate (Fig. 3.1H). The $\mathrm{S}^{+}$-form was never presented on the same position for more than two times in a row. We additionally cleaned the apparatus after every third trial. The time-interval between trials within a session was 10-30 s, depending on how fast the experimenter could re-bait the tube, change the tube positions and clean the apparatus, as well as how easily the subject was lured back to the starting position. We defined a trial as correct, if the subject extracted the food reward by only manipulating the $S^{+}$-form. If it manipulated an incorrect form, we noted the trial as incorrect and let the subject continue to explore the apparatus until it retrieved the reward or a maximum of $10 \mathrm{~min}$. Depending on the subject's motivation (i.e., actively moving around and easily lured to the start position), we conducted two sessions in a row with a short break of 5 min between sessions and a break of at least $30 \mathrm{~min}$ to a third session. We conducted a total of four to five sessions per individual and night, always taking the subject's motivation into account. After the subject had correctly chosen the $\mathrm{S}^{+}$-form for at least 24 out of 30 trials $(80 \%$-criterion over two consecutive sessions), we proceeded with the next experimental task, the visual reversal learning.

For the visual reversal learning, the former $\mathrm{S}^{+}$-form became unrewarded, and we chose one of the other three forms as new $S^{+}$. We started with the first session of this part 
in the same testing period as the last visual discrimination session. The rest of the testing procedure remained the same as in the visual discrimination until the $80 \%$-learning criterion was reached.

As the third experimental task, we conducted a spatial discrimination test. In this task, the shape and pattern of the forms became irrelevant, but only the position of the tube served as $\mathrm{S}^{+}$. We conducted the first session in the night following the last visual reversal learning session, which could be either during the same testing period, or when we recaptured the individual (days between tests: mean $=6.88 \pm 7.99$, $\max =27$ ). As in the visual tasks, we removed odor cues from the apparatus after every third trial. Additionally, we cleaned the tube opening, the frontal form, and the lid after each trial to remove any remainder of the food reward from the previous trial, if this specific tube was not positioned at the $\mathrm{S}^{+}$-position in the subsequent trial. The rest of the testing procedure remained the same until the $80 \%$-learning criterion was reached.

Finally, we conducted a spatial reversal learning test, with the former $\mathrm{S}^{+}$-position becoming non-rewarded and another position becoming the new $\mathrm{S}^{+}$. We chose a position on the other side of the plate to avoid a potential side preference. If, for example, the $\mathrm{S}^{+}$-position in the spatial discrimination was position 1, we chose the $\mathrm{S}^{+}$-position in the spatial reversal learning as either position 3 or 4 . As for the visual reversal learning, we conducted the first spatial reversal session in the same testing period as the last spatial discrimination session. The rest of the testing procedure remained the same as in the spatial discrimination until the $80 \%$-learning criterion was achieved.

For each trial, we measured the response latency and success latency similarly to the other experiments. For each session, we counted the number of correct and incorrect trials (errors) and calculated the mean response latency and the mean success latency. We used the number of errors across sessions as proxy for associative learning abilities in each task. For the overall performance per task, we used the total number of trials until the learning criterion was reached. As measure of flexibility, we calculated a transfer index (TI, Equation 1; (Rumbaugh and Pate 1984)) for the transitions with changing reward contingencies (i.e., from the visual discrimination to the visual reversal, from the visual reversal to the spatial discrimination, and from the spatial discrimination to the spatial reversal). Therefore, we used the last 15 trials of the previous task (pre-reversal 
performance) and the trial 2 to 15 of the following task (post-reversal performance). The first trial of the post-reversal session was excluded, since it primarily served as a cue that the initial reward contingency has changed.

\section{Equation $2 \quad \mathrm{TI}=\frac{\text { post-reversal performance }}{\text { pre-reversal performance }}$}

The sample sizes slightly decreased over the experimental tasks (Table S2-1), since we failed to recapture some individuals before they completed the repeated discrimination and reversal learning paradigm. Only $N=1$ individual failed to learn the reward contingency in the spatial discrimination. For $N=2$ individuals, we erroneously continued with the subsequent experimental task before the previous reward contingency was learned, and they were subsequently excluded from the analyses.

\section{Discrimination and reversal learning paradigm: Statistical analyses, learning across sessions}

To assess differences in associative learning abilities within each task, we used negative binomial mixed models with log link error function (function 'glmer.nb', 'MASS' package(23)) with the number of incorrect trials per session as response. For all the interspecific models, we set subject ID and session ID as random factors. We controlled for a possible influence of performance of the $\mathrm{S}^{+}$but did not include this variable as fixed factor if it did not affect the performance in the respective task. For the visual discrimination model (Table S2-8, model: a), we set species in interaction with session, sex, and age (log-transformed) as fixed factors. For the visual reversal learning model (Table S2-9, model: a), we set species, sex, age (log-transformed) and session as fixed factors. For the spatial discrimination model (Table S2-10, model: a), we set species in interaction with age (log-transformed), species in interaction with session, sex in interaction with session, and the $\mathrm{S}^{+}$as fixed factors. Since this model did not converge with standard settings, we set the optimizer to "bobyqa" and the maximum likelihood estimation to 100,000 iterations. For the spatial reversal learning model (Table S2-11, model: a), we set species in interaction with session, sex, age (log-transformed), and the $\mathrm{S}^{+}$as fixed factors. Since 
this model also did not converge with standard settings, we set the optimizer to "bobyqa" and the maximum likelihood estimation to 100,000 iterations.

\section{Discrimination and reversal learning paradigm: Statistical analyses, performance within tasks}

To assess variation in the overall performance within each task, we used linear gaussian models with the total number of trials (log-transformed) as response. For the interspecific models of the visual tasks (Table S2-8, Table S2-9, models: b), we set species, sex, and the mean age (log-transformed) between the first and the last session as fixed factors. For the spatial discrimination model (Table S2-10, model: b), we set species in interaction with the mean age (log-transformed), sex, and the $S^{+}$as fixed factors. For the spatial reversal learning model (Table S2-11, model: b), we set species, sex in interaction with the $\mathrm{S}^{+}$, and the mean age (log-transformed) as fixed factors. Due to small sample sizes, we calculated four less complex models per task for GML to model intraspecific variation in associative learning performances (Table S2-17, models: $\mathrm{f}, \mathrm{g}$, i, k). Therefore, we set sex and mean BMI (log-transformed) between the first and last session, sex and activity, sex and neophilia, and sex and the $\mathrm{S}^{+}$as respective fixed factors. For MBML, we calculated five models per task, reducing the number of fixed factors to one (Table S2-18, models: $\mathrm{f}, \mathrm{g}, \mathrm{i}, \mathrm{k}$ ).

\section{Discrimination and reversal learning paradigm: Statistical analyses, flexible learning between tasks: transfer index}

To assess variation in flexible learning between tasks, we modelled the TI using linear gaussian models with species, sex, and the mean age (log-transformed) as fixed factors (Table S2-9 - Table S2-11, models: c). Due to small sample sizes, we calculated three less complex models per task for GML to model intraspecific variation in flexible learning (Table S2-17, models: $h, j, 1)$. Therefore, we set sex and mean BMI (log-transformed) between the last pre-reversal and the first post-reversal session, sex and activity, and sex and neophilia as respective fixed factors. For MBML, we calculated four models per task, reducing the number of fixed factors to one (Table S2-18, models: $h, j, 1$ ). 
Additionally, we calculated Spearman rank correlations between the TIs and the number of trials to reach the posterior learning criteria, as well as the innovative propensity to estimate potential covariations between these flexible learning proxies.

\section{Plus maze: Experimental set-up}

To assess an individual's spatial learning abilities and spatial memory, we set up a plus maze with four arms $(40 \mathrm{~cm} \times 17 \mathrm{~cm} \times 17 \mathrm{~cm})$ leading to four terminal boxes (20 cm x $17 \mathrm{~cm} \times 17 \mathrm{~cm}$; Fig. 3.1B). Each terminal box contained a plastic lid in its back at the opposite side of its door, in which we placed a small piece of banana in case of the rewarding location. For an initial familiarization trial, we baited three terminal boxes and released the subject at the fourth box at the start arm. The familiarization trial started when we opened the door of the start box and ended either when the subject had eaten all the three food rewards or after a maximum of $15 \mathrm{~min}$. In the latter case, we repeated the familiarization trial either at the same or the following testing night when the individual showed higher motivation to participate in the task. After the subject had eaten the third reward, we distracted it briefly with a stick smeared with banana at the backside of the box, closed the door and released it again at the start box for the next trial or in its home cage when the test was done.

For the actual test session (15 successive trials), only one terminal box (= goal box) was baited. As goal box, we chose the box in which we caught the animal during the familiarization trial $\left(\mathrm{N}_{\text {straight }}=22, \mathrm{~N}_{\text {right }}=47, \mathrm{~N}_{\text {left }}=52\right)$. Since only the goal box was baited during the test session, we placed on top of each terminal box a piece of banana peel that was out of reach for the subject to control for olfactory stimuli. Similar to the familiarization trial, a test trial started when we opened the door of the start box and ended as soon as the subject had retrieved the food reward in the goal box. However, we stopped a trial after $10 \mathrm{~min}$, when the subject did not succeed. We stopped a session if the subject did not exit the start box within $10 \mathrm{~min}$ and continued the session either later in the night or in the following night. We additionally cleaned the maze after every third trial. We 
rotated the terminal boxes throughout the session to prevent the subject to follow potential odor cues left inside the goal box.

We counted how often a subject entered the wrong arm and/or box and summed it up to an error score per trial, which we defined as spatial learning performance. For the overall spatial performance, we used the mean sum of errors throughout the 15 trials. We tested a total of $N=121$ (96 GML and $25 \mathrm{MBML}$ ). We estimated the repeatability of the mean sum of errors by calculating the ICC for $N=21$ individuals (15 GML and six MBML), that repeated this test $(\mathrm{ICC}=0.412) . N=5$ individuals were subjected to this task in a previous study 11 - 53 months earlier (mean = 33.23 \pm 15.80; (Huebner et al. 2018)).

\section{Plus maze: Statistical analyses, spatial learning across trials}

To investigate variation in spatial learning, we modelled the sum of errors per trial in a negative binomial mixed model with log link error function (function 'glmer.nb', 'MASS' package(Brian et al. 2021)). Since the model did not converge with standard settings, we set the optimizer to "bobyqa" and the maximum likelihood estimation to 100,000 iterations. We set species in interaction with trial, sex and the goal box as fixed factors and subject ID and trial ID as random factors (Table S2-12, model: a).

\section{Plus maze: Statistical analyses, spatial memory}

To investigate variation in spatial memory, we modelled the mean sum of errors across the 15 trials (log-transformed) in a gaussian linear model. For the interspecific model, we set species, sex, age (log-transformed), and the goal box as fixed factors (Table S2-12, model: b). In the intraspecific model for GML (Table S2-17, model: m), we set sex, season, BMI (log-transformed), activity, neophilia and the goal box as fixed factors. For MBML, we calculated four less complex models (Table S2-18, model: $\mathrm{m}$ ) due to the small sample size in this species. We set sex and season, sex and BMI (log-transformed), sex and activity, sex and neophilia, and sex and the goal box as respective fixed factors. 


\section{Cylinder test: Experimental set-up}

To assess an individual's inhibitory control, we conducted a detour-reaching task using the cylinder test design(MacLean et al. 2014; Kabadayi et al. 2018). Prior to testing, we conducted a training session with an opaque cylinder. The food reward was placed in the center of the cylinder, invisible for the subject during its approach. To reach the reward, the subject had to take a detour and enter the cylinder by one of the open sides which were set in an $90^{\circ}$ angle from the approach direction onto the experimental platform within the subject's cage. We additionally removed odor cues after every fifth trial. We counted the number of correct and incorrect trials until a learning criterion was met that was defined as five consecutive trials taking the detour as a first response without prior touching or sniffing at the cylinder(MacLean et al. 2014). In some cases $(N=23,18.9 \%)$, we only noticed during the video analysis that this criterion was not entirely met. $N=15$ individuals did not reach the criterion in the conducted trials. $N=8$ individuals already reached the criterion earlier, but some more trials were conducted. This erroneous evaluation happened when an individual approached very quickly without directly touching the cylinder but sniffing at its very edge. In these cases, it could be difficult to directly classify the sniffing as happening only from the side, i.e., the entrance of the cylinder (correct) or as happening from the front (incorrect). However, whether the learning criterion was achieved or not did not affect performance in the subsequent testing session indicated by a model excluding these individuals (Table S2-13, model: a).

For the actual testing session of ten trials, we changed the cylinder to a transparent cylinder and repeated the cleaning every third trial, the rest of the experimental set-up was the same as in the training (Fig. 3.1E). Using a transparent cylinder, the subject could see the charcoal-colored piece of banana, while approaching the task. It could also smell the banana from the front through little holes in the center of the cylinder. Nevertheless, it had to inhibit an initial response to reach directly through the transparent barrier. Instead, it had to take a detour to one of the open sides to enter the cylinder and reach the reward, as in the training. We used the number of incorrect trials as proxy for inhibitory control. We tested a total of $N=110$ individuals (83 GML and $27 \mathrm{MBML}$ ). We estimated the repeatability of the used variables by calculating the ICC for $N=12$ individuals (seven GML and five MBML), that repeated this test (number training trials: $\mathrm{ICC}=0.556$, 
number incorrect test trials: $\mathrm{ICC}=0.141) . N=2$ individuals were subjected to this task in a previous study $34-41$ months ago (mean $=37.61 \pm 3.91$; (Huebner et al. 2018)).

\section{Cylinder test: Statistical analyses}

To investigate variation in inhibitory control, we calculated a negative binomial model with log link error function (function 'glm.nb', 'MASS' package(Brian et al. 2021)). We set the number of incorrect trials during the testing session as response, and species, sex, age (log-transformed) and the number of training trials as fixed factors in the interspecific model (Table S2-13, model: b). In the intraspecific model for GML (Table S2-17, model: $n$ ), we set sex, season, BMI (log-transformed), activity and neophilia as fixed factors. For MBML, we calculated four less complex models (Table S2-18, model: $n$ ) due to the low sample size for this species. We set sex and season, sex and BMI (log-transformed), sex and activity, and sex and neophilia as fixed factors, respectively.

Additionally, we calculated Spearman rank correlations between inhibitory control and the TIs, the number of trials to reach the learning criteria in the discrimination and reversal learning paradigm, as well as the innovative propensity to investigate potential covariations between these proxies for flexible learning abilities.

\section{String-pulling task: Experimental set-up, single-string set-up}

To assess an individual's means-end understanding, we conducted a string-pulling task in the single-string set-up(Jacobs and Osvath 2015). As string served a cable tie that was placed onto an external platform attached to the subject's cage just in front of the experimental platform within the cage. At the far end of the cable tie, we attached a piece of banana onto a small plate. The inner end of the cable tie reached $5 \mathrm{~cm}$ into the cage (Fig. 3.1G). Just before we placed the cable tie at its position, we lured the subject to the top wire in the center of the experimental platform. After positioning the cable tie, the subject had $20 \mathrm{~min}$ time to pull the end of the cable tie into reach to access the reward. We measured the response latency as duration from positioning the cable tie until the subject's approach and calculated the success latency as timespan between the response and reaching the 
reward. For subjects that did not succeed (non-solver), we set the success latency to the maximum time of the trial $(20 \mathrm{~min})$ plus the response latency. We used this success latency as proxy for means-end understanding. We tested a total of $N=114$ individuals (86 GML and 28 MBML). We estimated the repeatability of the variables by calculating the ICC for $N=11$ individuals (seven GML and four MGML), that repeated this test (response latency: ICC $=-0.137$, success latency: $\mathrm{ICC}=-0.192) . N=5$ individuals were subjected to this task in a previous study $11-53$ months ago (mean $=34.36 \pm 15.31$; (Huebner 2020)).

\section{String-pulling task: Experimental set-up, perpendicular strings set-up}

To assess an individual's goal directedness, we conducted a string-pulling task with a perpendicular strings set-up(Jacobs and Osvath 2015). To ensure that the animal had sufficient understanding of the string-pulling task itself, we conducted a training prior to testing. This training consisted of the means-end understanding test, that was repeated until the subject successfully pulled the string three times. If it continuously failed to pull the cable tie for three trials, we moved the cable tie $2 \mathrm{~cm}$ further into the cage and decreased the maximum trial length to $10 \mathrm{~min}(N=11)$. If the subject failed to pull the cable tie for another three trials, we moved the cable tie another $2 \mathrm{~cm}$ further into the cage. We continued with this procedure until the subject succeeded. In the subsequent trials, we changed the set-up to the original one, with the cable tie reaching $5 \mathrm{~cm}$ into the cage. For the actual goal directedness task, we positioned two identic cable ties with four grid cells distance (about $6 \mathrm{~cm}$ ) at the center of the platform. The food reward was only attached to one cable tie (correct string), but we attached a plate at the outer end of both cable ties. The position of the correct string (left or right) was randomly assigned but counterbalanced across a session of ten trials and changed every second to third trial. The trials started when the two cable ties were fully introduced and ended when the subject reached the reward or after a maximum of $10 \mathrm{~min}$. When the subject pulled the incorrect string first, we did not stop the trial, but allowed it to further explore the task until it pulled the correct string and reached the reward within the maximum trial duration. After each trial, we removed potential odor cues with 70\%-ethanol. 
In the goal directedness task, we counted the number of correct and incorrect trials across one session of ten trials. We considered a trial as correct if the subject did only manipulate the correct string and reached the reward. We considered a trial as incorrect if the subject did not succeed, or if it manipulated the incorrect string. We used the number of incorrect trials as proxy for goal directedness. We tested a total of $N=105$ individuals (83 GML and $22 \mathrm{MBML}$ ). We estimated the repeatability of the number of incorrect trials by calculating the ICC for $N=8$ individuals (six GML and two MBML), that repeated this test $(\mathrm{ICC}=0.119)$.

\section{String pulling task: Statistical analyses, means-end understanding}

To investigate interspecific variation in means-end understanding, we calculated a cox proportional-hazards model with the function Surv(success latency I solver) as proportional hazard and species, sex, and age (log-transformed) as fixed factors (Table S214, model: a). In the intraspecific model for GML (Table S2-17, model: o), we set sex in interaction with neophilia, season, BMI (log-transformed) and activity as fixed factors. For MBML, we calculated four less complex models (Table S2-18, model: o) because of the low sample size in this species. We set sex and season, sex and BMI (log-transformed), sex and activity, and sex and neophilia as fixed factors, respectively.

\section{String pulling task: Statistical analyses, goal-directedness}

To investigate variation in goal directedness, we calculated a poisson model with log link error function. In the interspecific model (Table S2-14, model: b), we set the number of incorrect trials as response and species, sex, and age (log-transformed) as fixed factors. In the intraspecific model for GML (Table S2-17, model: p), we set sex, season, BMI (log-transformed), activity and neophilia as fixed factors. For MBML, we calculated four less complex models (Table S2-18, model: p) due to the low sample size for this species. We set sex and season, sex and BMI (log-transformed), sex and activity, and sex and neophilia as fixed factors, respectively. 


\section{General intelligence}

Finally, we investigated general intelligence across, as well as within species. For the interspecific $G$-factor, we calculated two principal axis factor analyses, using the function 'fa' with the argument ' $\mathrm{fm}$ ' set to 'pa' ('psych' package). The first PAF contained the performance scores of individuals that completed all tests (excluding the spatial discrimination and the spatial reversal due to very low sample sizes in these tasks; $N=21 \mathrm{GML}, N=9 \mathrm{MBML})$. For the second PAF, we used the performance scores of individuals that completed all tests, except for the discrimination and reversal learning paradigm ( $N=76 \mathrm{GML}, N=19 \mathrm{MBML})$. We controlled for sphericity by applying Bartlett's test and for sampling adequacy by applying KMO.

For the intraspecific $g$-factor, we used the same (log-transformed) performance scores as for the $G$-factor. For each species separately, we calculated two PCAs per species. The first PCA per species contained the performance scores of all tests (excluding the spatial discrimination and the spatial reversal due to very low sample sizes in these tasks), which resulted in a data set of $N=23 \mathrm{GML}$ and $N=9$ MBML. For the second PCA per species, we excluded all performance scores of the repeated discrimination and reversal learning paradigm, obtaining a sample size of $N=76 \mathrm{GML}$ and $N=19$ MBML. For each PCA, we controlled for sphericity by applying Bartlett's test and for sampling adequacy by applying KMO.

\section{Supplemental results and discussion}

\section{Interspecific comparisons}

We report the statistic results of the models estimating interspecific variation in personality traits (Table S2-5) and cognitive abilities (Table S2-6 - Table S2-14), as well as the results of the factor analytical approach to estimate a G/g-factor (Table S2-15 - Table S2-16). 


\section{Intraspecific variation in performance in personality and cognitive tests}

Since we did not find systematic variation among sex or age within species performance, we briefly summarize only significant differences below. The complete results are presented for GML in Table S2-17, and for MBML in Table S2-18.

\section{Gray mouse lemurs}

Briefly, performance in the food extraction and persistence task, the visual discrimination and reversal learning paradigm, spatial discrimination, plus maze, cylinder test, and string-pulling task was not influenced by sex, BMI, or personality factors (Table S2-17).

In the open field test, male GML were more explorative than females (LM: $p=0.009$, Table S2-17, model: a) and individuals in less good body condition were slightly more neophilic than individuals with higher BMI $(p=0.041$, Table S2-17, model: b). Variation in the spatial reversal performance (Table S2-17, model: k) was explained by sex (LM: $p=0.002)$ and body condition $(p=0.026)$. Males reached the learning criterion after more trials than females. GML in worse body condition reached the learning criterion after more trials than those in better body condition. Variation in flexible learning from the visual reversal learning to the spatial discrimination (Table S2-17, model: j) was explained by sex (LM: $p=0.049$ ), with males reaching a higher TI than females. Variation in flexible learning from the spatial discrimination to the spatial reversal learning (Table S2-17, model: 1) was explained by sex in interaction with neophilia (LM: $p=0.022)$, with more neophilic females achieving lower TIs than less neophilic females, whereas this effect was reversed for males.

\section{Madame Berthe's mouse lemurs}

Performance in the personality tests, food extraction task, visual discrimination and visual reversal performance, spatial discrimination and spatial reversal performance, flexible learning from the visual reversal learning to the spatial discrimination and from the spatial discrimination to the spatial reversal learning, plus maze, cylinder test, and goal directedness was not influenced by sex, BMI, or personality factors (Table S2-18). 
Variation in persistence (Table S2-18, model: e) was explained by sex in interaction with BMI (LM: $p=0.011$ ). Females in better body condition were less persistent than those in worse body condition and for males, this effect was reversed. Variation in flexible learning from the visual discrimination to the visual reversal learning (Table S2-18, model: h) was explained by sex (LM: $p=0.029)$, with males achieving lower TIs than females. Variation in means-end understanding (Table S2-18, model: o) was predicted by BMI (cox PHM: $p=0.016$ ), with MBML in better body condition solving the string-pulling task in the single-string set-up faster than those in worse body condition.

\section{Discussion: Intra-specific variation in performance in personality and cognitive tests}

Concerning individual characteristics influencing performance in personality and cognitive tests, we found minor and inconsistent sex or age effects across tasks. First, males and females differed only in four out of 22 performance measures (in two cognitive tests and in two personality tests). Second, we controlled for potential effects of motivational state to participate in tests by estimating an individual's BMI(Sol et al. 2012; Cauchoix et al. 2020), but BMI was positively correlated with cognitive performance in only two out of 22 performance measures, suggesting no systematic link between test performance and an individual's current motivational state. Third, learning abilities partially covaried with age but not consistently across tasks and species. In our study, we mainly tested rather young individuals of a limited age range, with only 10 individuals being 4 yrs. or older (mean age $=1.21 \pm 1.34$ yrs.). Previous studies reported a positive correlation between age and learning abilities, and an increased impairment of cognitive abilities in elderly individuals(Bonté et al. 2014; Franks and Thorogood 2018; Hopkins et al. 2020). However, previous studies on mouse lemurs were conducted on captive individuals of larger age ranges (up to 13 yrs.) with a categorization of "elderly" or "aged" individuals starting at an age of five or six years(Languille et al. 2012). Compared to younger individuals, aged mouse lemurs express deficits in memory, flexible learning, and spatial abilities, while simple associative abilities seem to be preserved(Trouche et al. 2010; Picq et al. 2012). Wild mouse lemurs have a relatively short modal life expectancy of about three years and have been previously reported to not exhibit functional senescence, unlike their captive 
conspecifics(Hämäläinen et al. 2014a). Thus, potential age effects in cognitive performance might be difficult to demonstrate in wild mouse lemurs and the here reported correlations do not allow for firm conclusions about a relationship between an individual's age and its cognitive performance.

Regarding the personality traits, neither neophilia, nor activity covaried with cognitive performance levels in either GML or MBML. In several prior studies, performance scores were confounded by variation in personality traits(Tebbich et al. 2012; Guenther et al. 2014). However, these links are by far not consistent across studies, emphasizing the importance to control for such non-cognitive factors(Ducatez et al. 2014a; Griffin et al. 2015; Guillette et al. 2015; Logan 2016; Huebner et al. 2018; Henke-von der Malsburg and Fichtel 2018; Rasolofoniaina et al. 2020; Schubiger et al. 2020).

\section{Supplemental tables}

Table S2-1 Sample sizes per species and sex for the single tasks, as well as the measured personality or cognitive trait, and the number of individuals that were not naïve to the respective experimental task. Sample sizes differ between tasks as it was not possible to recapture all individuals until they had completed all tasks of the test battery

\begin{tabular}{|c|c|c|c|c|c|c|c|c|}
\hline \multirow{2}{*}{ task } & \multirow{2}{*}{ measured trait } & \multicolumn{2}{|c|}{ GML } & \multirow{2}{*}{$\begin{array}{c}\text { not } \\
\text { naïve }\end{array}$} & \multicolumn{2}{|c|}{ MBML } & \multirow{2}{*}{\begin{tabular}{|c|} 
not \\
naïve
\end{tabular}} & \multirow{2}{*}{ total } \\
\hline & & $\mathbf{F}$ & $\mathbf{M}$ & & $\mathbf{F}$ & $\mathbf{M}$ & & \\
\hline open field test & activity & 51 & 67 & 15 & 19 & 13 & 1 & 150 \\
\hline novel object test & neophilia & 51 & 67 & 0 & 19 & 13 & 1 & 150 \\
\hline food extraction task & $\begin{array}{l}\text { innovative } \\
\text { propensity }\end{array}$ & 36 & 47 & 5 & 18 & 11 & 0 & 112 \\
\hline persistence test & persistence & 35 & 47 & 0 & 17 & 9 & 0 & 108 \\
\hline $\begin{array}{l}\text { visual } \\
\text { discrimination }\end{array}$ & $\begin{array}{l}\text { associative } \\
\text { learning }\end{array}$ & 14 & 11 & 0 & 8 & 6 & 0 & 39 \\
\hline visual reversal & $\begin{array}{l}\text { associative/ } \\
\text { flexible learning }\end{array}$ & 14 & 11 & 0 & 7 & 5 & 0 & 37 \\
\hline $\begin{array}{l}\text { spatial } \\
\text { discrimination }\end{array}$ & $\begin{array}{l}\text { associative/ } \\
\text { flexible learning }\end{array}$ & 12 & 11 & 0 & 4 & 5 & 0 & 32 \\
\hline spatial reversal & $\begin{array}{l}\text { associative/ } \\
\text { flexible learning }\end{array}$ & 11 & 10 & 0 & 2 & 3 & 0 & 26 \\
\hline plus maze & spatial memory & 39 & 57 & 5 & 15 & 10 & 0 & 121 \\
\hline cylinder test & $\begin{array}{l}\text { inhibitory } \\
\text { control }\end{array}$ & 37 & 46 & 2 & 17 & 10 & 0 & 110 \\
\hline string-pulling task & $\begin{array}{l}\text { means-end } \\
\text { understanding }\end{array}$ & 37 & 49 & 5 & 17 & 11 & 0 & 114 \\
\hline $\begin{array}{l}\text { perpendicular } \\
\text { strings }\end{array}$ & $\begin{array}{l}\text { goal } \\
\text { directedness }\end{array}$ & 36 & 47 & 0 & 15 & 7 & 0 & 105 \\
\hline
\end{tabular}


Table S2-2 Intraclass correlation coefficients (ICC) estimating the interobserver reliability of the measured behaviors for $10 \%$ of the analyzed videos per task

\begin{tabular}{|c|c|c|}
\hline measured behavior & ICC per behavior & ICC per test \\
\hline open field test & & 0.989 \\
\hline latency enter & $>0.999$ & \\
\hline stationary & 0.831 & \\
\hline locomotion & 0.866 & \\
\hline cells traversed & 0.990 & \\
\hline latency grid & 0.918 & \\
\hline grid cell changes & 0.995 & \\
\hline jumps & 0.914 & \\
\hline rears & 0.778 & \\
\hline dips & 0.396 & \\
\hline novel object test & & 0.905 \\
\hline approach latency & 0.971 & \\
\hline contact latency & 0.974 & \\
\hline approach speed & 0.862 & \\
\hline contact frequency & 0.910 & \\
\hline food extraction task & & $>0.999$ \\
\hline response latency & $>0.999$ & \\
\hline success latency & $>0.999$ & \\
\hline number of successes & 0.837 & \\
\hline persistence test & & 0.957 \\
\hline duration in contact & 0.906 & \\
\hline duration manipulating & 0.992 & \\
\hline solver & 1.000 & \\
\hline \multicolumn{2}{|c|}{ repeated discrimination and reversal learning paradigm } & 0.999 \\
\hline response latency & $>0.999$ & \\
\hline success latency & 0.997 & \\
\hline number of incorrect trials & 0.937 & \\
\hline \multicolumn{2}{|l|}{ plus maze } & 0.986 \\
\hline latency to enter correct arm per trial & 0.984 & \\
\hline sum of errors per trial & 0.877 & \\
\hline mean sum of errors per session & 0.961 & \\
\hline \multicolumn{2}{|l|}{ cylinder test } & 0.802 \\
\hline number of incorrect trials & 0.802 & \\
\hline \multicolumn{2}{|l|}{ string-pulling task single-string set-up } & $>0.999$ \\
\hline response latency & $>0.999$ & \\
\hline success latency & $>0.999$ & \\
\hline \multirow{2}{*}{\multicolumn{2}{|c|}{ string-pulling task perpendicular strings set-up }} & 1.000 \\
\hline & 1.000 & \\
\hline
\end{tabular}


Table S2-3 Repeatability of the personality tests. Intraclass correlation coefficients were calculated between the first and second test, between the first, second, and third test, and between the first, second, third, and fourth test. Sample sizes are given in parentheses

\begin{tabular}{|l|ccc|}
\hline behavior & test $\mathbf{1 - 2}(\mathbf{N}=\mathbf{1 0 4})$ & test $\mathbf{1 - 3}(\mathbf{N}=\mathbf{5 2})$ & test 1-4 $(\mathbf{N}=\mathbf{2 9})$ \\
\hline latency enter & -0.103 & -0.040 & -0.061 \\
locomotion & 0.343 & 0.409 & 0.415 \\
cells traversed & 0.381 & 0.423 & 0.358 \\
rears* & 0.365 & 0.275 & 0.269 \\
dips* & 0.132 & 0.059 & 0.076 \\
contact latency & 0.022 & -0.046 & 0.017 \\
approach speed & 0.158 & 0.044 & 0.073 \\
contact frequency & 0.106 & 0.002 & 0.036 \\
\hline
\end{tabular}

*log-transformed variables

Table S2-4 Loadings of the principal component analyses (varimax rotation) of the first and second open field test, including eigenvalues and explained variances per rotated component (RC), as well as the Kaiser-MeyerOlkin factor (KMO) and statistics of the Bartlett's test of sphericity

\begin{tabular}{|l|rr|rr|}
\hline \multirow{2}{*}{ behavior } & \multicolumn{2}{|c|}{ test 1 } & \multicolumn{2}{c|}{ test 2 } \\
\cline { 2 - 5 } & RC1 & RC2 & RC1 & RC2 \\
\hline latency enter* & -0.486 & -0.028 & -0.424 & -0.142 \\
locomotion & 0.898 & 0.056 & 0.916 & 0.042 \\
cells traversed & 0.904 & 0.138 & 0.912 & 0.107 \\
rears* & 0.260 & 0.773 & 0.306 & 0.787 \\
dips* & -0.073 & 0.868 & -0.018 & 0.892 \\
\hline eigenvalue & 1.93 & 1.37 & 1.95 & 1.45 \\
explained variance & 0.58 & 0.42 & 0.57 & 0.43 \\
\hline KMO & \multicolumn{2}{|c|}{0.555} & \multicolumn{2}{c|}{0.547} \\
\hline Bartlett's test & \multicolumn{2}{|c|}{$\mathrm{X}^{2}=203.509$} & \multicolumn{2}{c|}{$\mathrm{X}^{2}=151.463$} \\
& \multicolumn{2}{|c|}{$\mathrm{df}=10$} & $\mathrm{df}=10$ \\
\hline
\end{tabular}

${ }^{*} \log$-transformed variables 
Table S2-5 Personality traits. Given are estimates, test-statistics (ts), p-value and degrees of freedom (df) of the full-null-model comparison (bold), effect sizes $\left(\mathrm{R}^{2}\right)$, as well as estimates and standard errors (SE), teststatistics and p-values of the individual predictors. GML: gray mouse lemur, M: male

\begin{tabular}{|c|c|c|c|c|c|c|}
\hline model ID & $\begin{array}{l}\text { response } \\
\text { fixed factors }\end{array}$ & estimate $\pm \mathrm{SE}$ & ts & p & df & $\mathbf{R}^{2}$ \\
\hline a) & $\begin{array}{l}\text { activity } \\
\text { species * sex } \\
\text { age }\end{array}$ & $\begin{array}{l}\text { GML: }-38.45 \pm 10.90 \\
\text { M: }-18.26 \pm 14.58 \\
\text { GML:M: } 39.35 \pm 16.41 \\
-2.22 \pm 3.34\end{array}$ & $\begin{array}{l}3.99 \\
5.75\end{array}$ & $\begin{array}{l}\mathbf{0 . 0 0 4} \\
0.018\end{array}$ & 4 & 0.074 \\
\hline b) & $\begin{array}{l}\text { neophilia } \\
\text { species } \\
\text { sex } \\
\text { age } \\
\text { zero-part: sex }\end{array}$ & $\begin{array}{l}\text { GML: } 0.11 \pm 0.16 \\
\text { M: } 0.04 \pm 0.14 \\
-0.03 \pm 0.07 \\
\text { F: }-0.93 \pm 0.32 \\
\text { M: }-1.49 \pm 0.71\end{array}$ & $\begin{array}{r}8.56 \\
0.69 \\
0.27 \\
-0.49 \\
-2.87 \\
-2.12\end{array}$ & $\begin{array}{l}\mathbf{0 . 0 7 3} \\
0.489 \\
0.791 \\
0.621 \\
0.004 \\
0.034\end{array}$ & 4 & 0.056 \\
\hline
\end{tabular}

Table S2-6 Foraging extraction task. Given are estimates, test-statistics (ts), p-value and degrees of freedom (df) of the full-null-model comparison (bold), effect sizes $\left(\mathrm{R}^{2}\right)$, as well as estimates and standard errors (SE), test-statistics and p-values of the individual predictors. GML: gray mouse lemur, M: male

\begin{tabular}{|c|c|c|c|c|c|c|}
\hline model ID & $\begin{array}{l}\text { response } \\
\text { fixed factors }\end{array}$ & estimate $\pm S E$ & ts & $\mathbf{p}$ & df & $\mathbf{R}^{2}$ \\
\hline \multirow[t]{5}{*}{ a) } & \multicolumn{6}{|c|}{ innovation speed } \\
\hline & \multicolumn{2}{|c|}{ Surv(success latency | solver) } & 11.37 & 0.010 & 3 & 0.102 \\
\hline & species & GML: $0.61 \pm 0.24$ & 2.49 & 0.013 & & \\
\hline & $\operatorname{sex}$ & M: $0.41 \pm 0.20$ & 2.02 & 0.044 & & \\
\hline & age & $0.13 \pm 0.10$ & 1.26 & 0.209 & & \\
\hline \multirow[t]{5}{*}{ b) } & \multicolumn{6}{|c|}{ innovation propensity } \\
\hline & \multicolumn{2}{|c|}{$\mathrm{N}$ opened wells } & 8.39 & 0.039 & 3 & 0.073 \\
\hline & species & GML: $0.28 \pm 0.10$ & 7.64 & 0.006 & & \\
\hline & $\operatorname{sex}$ & M: $0.04 \pm 0.08$ & 0.181 & 0.670 & & \\
\hline & age & $0.01 \pm 0.04$ & 0.03 & 0.874 & & \\
\hline
\end{tabular}


Table S2-7 Persistence, a) total sample size, b) excluding $N=17$ individuals that received additional training. Given are estimates, test-statistics (ts), p-value and degrees of freedom (df) of the full-null-model comparison (bold), effect sizes $\left(R^{2}\right)$, as well as estimates and standard errors (SE), test-statistics and p-values of the individual predictors. GML: gray mouse lemur, M: male

\begin{tabular}{|l|llrrrr|}
\hline \multirow{2}{*}{ model ID } & $\begin{array}{l}\text { response } \\
\text { fixed factors }\end{array}$ & estimate $\pm \mathbf{S E}$ & ts & $\mathbf{p}$ & $\mathbf{d f}$ & $\mathbf{R}^{\mathbf{2}}$ \\
\hline a) & persistence, $\mathbf{N}=\mathbf{1 0 8}$ & $\mathbf{9 . 4 7}$ & $<\mathbf{0 . 0 0 1}$ & $\mathbf{3}$ & $\mathbf{0 . 1 9 2}$ \\
& species & GML: $0.15 \pm 0.03$ & 25.56 & $<0.001$ & & \\
& sex & M: $-0.03 \pm 0.03$ & 1.68 & 0.198 & & \\
& age & $-0.02 \pm 0.01$ & 2.92 & 0.090 & & \\
\hline b) & persistence, $\mathbf{N}=\mathbf{9 1}$ & $\mathbf{6 . 3 6}$ & $<\mathbf{0 . 0 0 1}$ & $\mathbf{3}$ & $\mathbf{0 . 1 5 2}$ \\
& species & GML: $0.15 \pm 0.04$ & 16.52 & $<0.001$ & & \\
& sex & M: $-0.04 \pm 0.03$ & 1.98 & 0.163 & & \\
& age & $-0.02 \pm 0.01$ & 2.39 & 0.126 & & \\
\hline
\end{tabular}

Table S2-8 Visual discrimination. Given are estimates, test-statistics (ts), p-value and degrees of freedom (df) of the full-null-model comparison (bold), effect sizes $\left(\mathrm{R}^{2}\right)$, as well as estimates and standard errors (SE), teststatistics and p-values of the individual predictors. GML: gray mouse lemur, M: male

\begin{tabular}{|c|c|c|c|c|c|c|}
\hline model ID & $\begin{array}{l}\text { response } \\
\text { fixed factors }\end{array}$ & estimate $\pm \mathrm{SE}$ & ts & p & df & $\mathbf{R}^{2}$ \\
\hline a) & $\begin{array}{l}\text { visual discriminati } \\
\mathbf{N} \text { incorrect trials } \\
\text { species }{ }^{*} \text { session } \\
\text { sex } \\
\text { age }\end{array}$ & $\begin{array}{l}\text { learning } \\
\text { GML: }-0.29 \pm 0.19 \\
\text { session: }-0.25 \pm 0.07 \\
\text { session:GML: } 0.14 \pm 0.06 \\
\text { M: } 0.18 \pm 0.11 \\
0.14 \pm 0.06\end{array}$ & $\begin{array}{r}20.99 \\
5.46\end{array}$ & $\begin{array}{r}<0.001 \\
0.020\end{array}$ & 5 & 0.088 \\
\hline b) & $\begin{array}{l}\text { visual discriminati } \\
\mathbf{N} \text { total trials } \\
\text { species } \\
\text { sex } \\
\text { age }\end{array}$ & $\begin{array}{l}\text { performance } \\
\text { GML: } 0.36 \pm 0.13 \\
\text { M: } 0.10 \pm 0.13 \\
0.18 \pm 0.07\end{array}$ & $\begin{array}{l}\mathbf{5 . 1 6} \\
7.70 \\
0.56 \\
7.81\end{array}$ & $\begin{array}{l}\mathbf{0 . 0 0 5} \\
0.009 \\
0.458 \\
0.008\end{array}$ & 3 & 0.247 \\
\hline
\end{tabular}


Table S2-9 Visual reversal and flexible learning. Given are estimates, test-statistics (ts), p-value and degrees of freedom (df) of the full-null-model comparison (bold), effect sizes $\left(\mathrm{R}^{2}\right)$, as well as estimates and standard errors (SE), test-statistics and p-values of the individual predictors. GML: gray mouse lemur, M: male

\begin{tabular}{|c|c|c|c|c|c|c|}
\hline model ID & $\begin{array}{l}\text { response } \\
\text { fixed factors }\end{array}$ & estimate $\pm \mathrm{SE}$ & ts & p & df & $\mathbf{R}^{2}$ \\
\hline a) & $\begin{array}{l}\text { visual reversal lea } \\
\mathrm{N} \text { incorrect trials } \\
\text { species } \\
\text { sex } \\
\text { age } \\
\text { session }\end{array}$ & $\begin{array}{l}\text { rning } \\
\text { GML: } 0.42 \pm 0.12 \\
\text { M: } 0.04 \pm 0.11 \\
-0.14 \pm 0.06 \\
-0.38 \pm 0.03\end{array}$ & $\begin{array}{r}41.57 \\
10.31 \\
0.10 \\
5.61 \\
24.75\end{array}$ & $\begin{array}{r}<0.001 \\
0.001 \\
0.758 \\
0.018 \\
<0.001\end{array}$ & 4 & 0.234 \\
\hline b) & $\begin{array}{l}\text { visual reversal per } \\
\mathbf{N} \text { total trials } \\
\text { species } \\
\text { sex } \\
\text { age }\end{array}$ & $\begin{array}{l}\text { formance } \\
\text { GML: } 0.31 \pm 0.10 \\
\text { M: } 0.05 \pm 0.10 \\
-0.07 \pm 0.05\end{array}$ & $\begin{array}{r}4.30 \\
10.29 \\
0.26 \\
1.83\end{array}$ & $\begin{array}{l}\mathbf{0 . 0 1 1} \\
0.003 \\
0.614 \\
0.186\end{array}$ & 3 & 0.216 \\
\hline c) & $\begin{array}{l}\text { flexible learning ( } \\
\text { TI } \\
\text { species } \\
\text { sex } \\
\text { age }\end{array}$ & $\begin{array}{l}\text { isual cues) } \\
\text { GML: } 0.09 \pm 0.09 \\
\text { M: }-0.12 \pm 0.09 \\
0.08 \pm 0.04\end{array}$ & $\begin{array}{l}2.87 \\
1.06 \\
1.77 \\
3.96\end{array}$ & $\begin{array}{l}\mathbf{0 . 0 5 0} \\
0.310 \\
0.192 \\
0.054\end{array}$ & 3 & 0.128 \\
\hline
\end{tabular}


Table S2-10 Spatial discrimination and flexible learning. Given are estimates, test-statistics (ts), p-value and degrees of freedom (df) of the full-null-model comparison (bold), effect sizes $\left(\mathrm{R}^{2}\right)$, as well as estimates and standard errors (SE), test-statistics and p-values of the individual predictors. GML: gray mouse lemur, M: male

\begin{tabular}{|c|c|c|c|c|c|c|}
\hline model ID & $\begin{array}{l}\text { response } \\
\text { fixed factors }\end{array}$ & estimate $\pm \mathrm{SE}$ & ts & p & df & $\mathbf{R}^{2}$ \\
\hline \multirow[t]{12}{*}{ a) } & \multicolumn{6}{|c|}{ spatial discrimination learning } \\
\hline & \multicolumn{2}{|c|}{$\mathrm{N}$ incorrect trials } & 73.17 & $<0.001$ & 10 & 0.412 \\
\hline & \multirow[t]{3}{*}{ species ${ }^{*}$ session } & GML: $-0.58 \pm 0.25$ & 10.02 & 0.002 & & \\
\hline & & session: $-0.33 \pm 0.07$ & & & & \\
\hline & & session:GML: $0.21 \pm 0.07$ & & & & \\
\hline & \multirow[t]{2}{*}{ sex ${ }^{*}$ session } & M: $0.14 \pm 0.23$ & 10.71 & 0.001 & & \\
\hline & & session:M: $-0.17 \pm 0.05$ & & & & \\
\hline & \multirow[t]{2}{*}{ species * age } & age: $-0.57 \pm 0.20$ & 7.83 & 0.005 & & \\
\hline & & age:GML: $0.68 \pm 0.24$ & & & & \\
\hline & \multirow[t]{3}{*}{$S^{ \pm}$} & position $2: 1.47 \pm 0.33$ & 27.91 & $<0.001$ & & \\
\hline & & position $3: 1.56 \pm 0.28$ & & & & \\
\hline & & position $4: 1.34 \pm 0.26$ & & & & \\
\hline \multirow[t]{9}{*}{ b) } & \multicolumn{6}{|c|}{ spatial discrimination performance } \\
\hline & \multicolumn{2}{|l|}{$\mathrm{N}$ total trials } & 3.48 & 0.010 & 7 & 0.359 \\
\hline & \multirow[t]{3}{*}{ species * age } & GML: $0.05 \pm 0.18$ & 4.58 & 0.043 & & \\
\hline & & age: $-0.30 \pm 0.16$ & & & & \\
\hline & & age:GML: $0.44 \pm 0.20$ & & & & \\
\hline & \multirow{4}{*}{$\begin{array}{l}\text { Sex } \\
S^{ \pm}\end{array}$} & M: $-0.02 \pm 0.19$ & 0.01 & 0.931 & & \\
\hline & & position 2: $0.96 \pm 0.29$ & 6.42 & 0.002 & & \\
\hline & & position 3: $0.93 \pm 0.24$ & & & & \\
\hline & & position $4: 0.74 \pm 0.23$ & & & & \\
\hline \multirow[t]{5}{*}{ c) } & \multicolumn{6}{|c|}{ flexible learning (cue change) } \\
\hline & \multicolumn{2}{|l|}{ TI } & 3.50 & 0.027 & 3 & 0.181 \\
\hline & species & GML: $0.16 \pm 0.09$ & 3.64 & 0.066 & & \\
\hline & sex & M: $0.22 \pm 0.08$ & 6.71 & 0.014 & & \\
\hline & age & $0.02 \pm 0.04$ & 0.17 & 0.680 & & \\
\hline
\end{tabular}


Table S2-11 Spatial reversal and flexible learning. Given are estimates, test-statistics (ts), p-value and degrees of freedom (df) of the full-null-model comparison (bold), effect sizes $\left(\mathrm{R}^{2}\right)$, as well as estimates and standard errors (SE), test-statistics and p-values of the individual predictors. GML: gray mouse lemur, M: male

\begin{tabular}{|c|c|c|c|c|c|c|}
\hline model ID & $\begin{array}{l}\text { response } \\
\text { fixed factors }\end{array}$ & estimate $\pm S E$ & ts & p & df & $\mathbf{R}^{2}$ \\
\hline a) & $\begin{array}{l}\text { spatial reversal } \\
\text { N incorrect tria } \\
\text { species } \\
\text { session } \\
\text { sex } \\
\text { age } \\
\mathrm{S}^{ \pm}\end{array}$ & $\begin{array}{l}\text { earning } \\
\text { GML: }-0.86 \pm 0.27 \\
\text { session: }-0.19 \pm 0.05 \\
\text { session:GML: } 0.13 \pm 0.05 \\
\text { M: } 0.35 \pm 0.16 \\
0.006 \pm 0.07 \\
\text { position } 2: 0.13 \pm 0.25 \\
\text { position } 3: 0.64 \pm 0.22 \\
\text { position } 4: 0.28 \pm 0.24\end{array}$ & $\begin{array}{l}4.51 \\
0.01 \\
8.24\end{array}$ & $\begin{array}{l}0.034 \\
0.931 \\
0.041\end{array}$ & 10 & 0.220 \\
\hline b) & $\begin{array}{l}\text { spatial reversal } \\
\text { N total trials } \\
\text { species } \\
\text { sex }{ }^{ \pm} S^{ \pm} \\
\text {age }\end{array}$ & $\begin{array}{l}\text { erformance } \\
\text { GML: }-0.24 \pm 0.21 \\
\text { M: } 0.53 \pm 0.29 \\
\text { position 2: } 0.61 \pm 0.42 \\
\text { position 2:M: }-0.42 \pm 0.53 \\
\text { position 3: } 0.23 \pm 0.26 \\
\text { position 3:M: } 0.95 \pm 0.40 \\
\text { position 4: } 0.45 \pm 0.5 \\
\text { position 4:M: }-0.58 \pm 0.38 \\
-0.14 \pm 0.09\end{array}$ & $\begin{array}{l}6.19 \\
1.33 \\
5.05\end{array}$ & $\begin{array}{r}<0.001 \\
0.267 \\
0.012\end{array}$ & 9 & 0.651 \\
\hline c) & $\begin{array}{l}\text { flexible learning } \\
\text { TI } \\
\text { species } \\
\text { sex } \\
\text { age }\end{array}$ & $\begin{array}{l}\text { spatial cues) } \\
\text { GML: } 0.39 \pm 0.10 \\
\text { M: }-0.13 \pm 0.09 \\
0.03 \pm 0.05\end{array}$ & $\begin{array}{r}6.90 \\
16.18 \\
2.01 \\
0.49\end{array}$ & $\begin{array}{r}\mathbf{0 . 0 0 1} \\
<0.001 \\
0.168 \\
0.492\end{array}$ & 3 & 0.364 \\
\hline
\end{tabular}


Table S2-12 Spatial memory. Given are estimates, test-statistics (ts), p-value and degrees of freedom (df) of the full-null-model comparison (bold), effect sizes $\left(\mathrm{R}^{2}\right)$, as well as estimates and standard errors (SE), teststatistics and p-values of the individual predictors. GML: gray mouse lemur, M: male

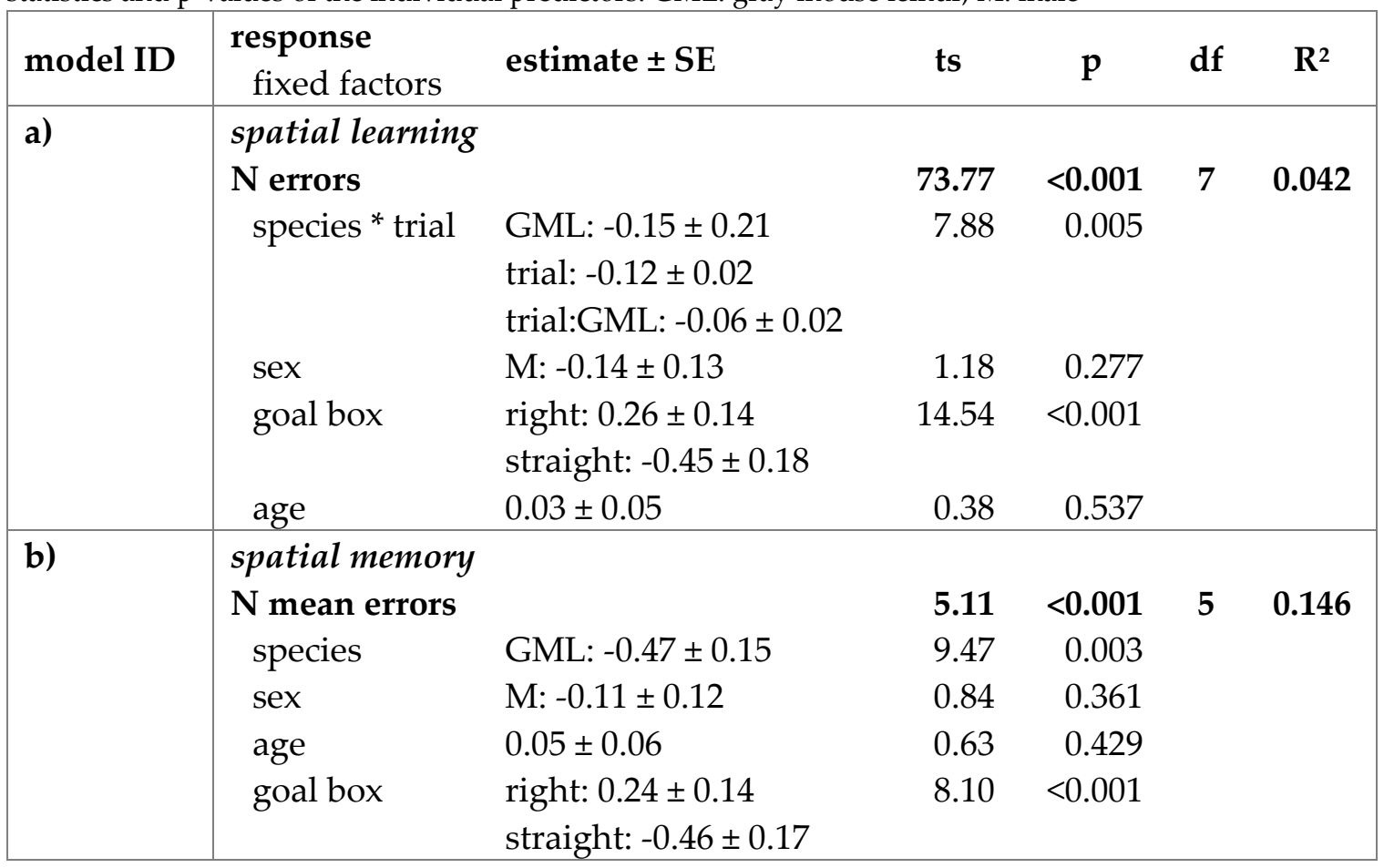

Table S2-13 Inhibitory control, a) excluding N = 21 individuals that did not meet the learning criterion in the prior training session, b) total sample size. Given are estimates, test-statistics (ts), p-value and degrees of freedom (df) of the full-null-model comparison (bold), effect sizes $\left(\mathrm{R}^{2}\right)$, as well as estimates and standard errors (SE), test-statistics and p-values of the individual predictors. GML: gray mouse lemur, M: male

\begin{tabular}{|c|c|c|c|c|c|c|}
\hline model ID & $\begin{array}{l}\text { response } \\
\text { fixed factors }\end{array}$ & estimate $\pm \mathrm{SE}$ & ts & p & df & $\mathbf{R}^{2}$ \\
\hline a) & $\begin{array}{l}\text { inhibitory control, } \\
\mathrm{N} \text { incorrect trials } \\
\text { species } \\
\text { sex } \\
\text { age } \\
\mathrm{N} \text { trials training }\end{array}$ & $\begin{array}{l}N=87 \\
\text { GML: }-0.16 \pm 0.17 \\
\text { M: }-0.09 \pm 0.14 \\
-0.04 \pm 0.07 \\
0.02 \pm 0.01\end{array}$ & $\begin{array}{l}8.22 \\
0.85 \\
0.38 \\
0.27 \\
6.85\end{array}$ & $\begin{array}{l}\mathbf{0 . 0 8 4} \\
0.356 \\
0.538 \\
0.598 \\
0.009\end{array}$ & 4 & 0.081 \\
\hline b) & $\begin{array}{l}\text { inhibitory control, } \\
\mathrm{N} \text { incorrect trials } \\
\text { species } \\
\text { sex } \\
\text { age } \\
\mathrm{N} \text { trials training }\end{array}$ & $\begin{array}{l}N=110 \\
\text { GML: }-0.21 \pm 0.14 \\
\text { M: } 0.03 \pm 0.12 \\
-0.06 \pm 0.06 \\
0.02 \pm 0.01\end{array}$ & $\begin{array}{r}\mathbf{1 0 . 3 4} \\
2.34 \\
0.08 \\
0.99 \\
7.98\end{array}$ & $\begin{array}{l}\mathbf{0 . 0 3 5} \\
0.126 \\
0.783 \\
0.319 \\
0.005\end{array}$ & 4 & 0.082 \\
\hline
\end{tabular}


Table S2-14 Means-end understanding and goal directedness. Given are estimates, test-statistics (ts), p-value and degrees of freedom (df) of the full-null-model comparison (bold), effect sizes $\left(\mathrm{R}^{2}\right)$, as well as estimates and standard errors (SE), test-statistics and p-values of the individual predictors. GML: gray mouse lemur, M: male

\begin{tabular}{|l|llcccc|}
\hline \multirow{2}{*}{ model ID } & $\begin{array}{l}\text { response } \\
\text { fixed factors }\end{array}$ & estimate \pm SE & ts & $\mathbf{p}$ & $\mathbf{d f}$ & $\mathbf{R}^{\mathbf{2}}$ \\
\hline a) & means-end understanding & & & & \\
& Surv(success latency l solver) & $\mathbf{1 . 9 8}$ & $\mathbf{0 . 6 0 0}$ & $\mathbf{3}$ & $\mathbf{0 . 0 1 8}$ \\
& species & GML: $0.33 \pm 0.24$ & 2.01 & 0.156 & & \\
& sex & M: $0.01 \pm 0.20$ & 0.00 & 0.986 & & \\
& age & $0.01 \pm 0.09$ & 0.01 & 0.942 & & \\
\hline b) & goal directedness & & & & & \\
& N incorrect trials & & $\mathbf{0 . 6 7}$ & $\mathbf{0 . 8 7 4}$ & $\mathbf{3}$ & $\mathbf{0 . 0 0 6}$ \\
& species & GML: $-0.05 \pm 0.10$ & 0.25 & 0.621 & & \\
& sex & M: $0.06 \pm 0.09$ & 0.44 & 0.508 & & \\
& age & $0.01 \pm 0.04$ & 0.10 & 0.747 & & \\
\hline
\end{tabular}

Table S2-15 General intelligence on reduced set of tests. Interspecific G loadings of the PAF, as well as intraspecific $g$ loadings for gray mouse lemurs (GML) and Madame Berthe's mouse lemurs (MBML). For each factor and component, the squared factor (SS) loadings or eigenvalue, and the proportion of explained variance are given. For each analysis, the sample size $(\mathrm{N})$, the Kaiser-Meyer-Olkin (KMO) index, and the statistics for the Bartlett's test of sphericity are given

\begin{tabular}{|l|ccc|}
\hline cognitive performance & $\begin{array}{c}\text { G loadings } \\
\left.\mathbf{( N G M L}=\mathbf{7 6}, \mathbf{N}_{\text {MBML }} \mathbf{1 9 )}\right)\end{array}$ & $\begin{array}{c}\text { g loadings } \\
\text { GML (N = 76) }\end{array}$ & $\begin{array}{c}\text { g loadings } \\
\text { MBML (N = 19) }\end{array}$ \\
\hline FE: success latency & 0.54 & -0.57 & -0.31 \\
FEP: persistence & -0.02 & -0.43 & 0.47 \\
CT: N incorrect trials & -0.02 & 0.07 & 0.21 \\
SP1: success latency & -0.22 & 0.29 & 0.53 \\
SP2: N incorrect trials & -0.07 & 0.21 & -0.26 \\
PM: N mean errors & 0.50 & -0.60 & -0.54 \\
\hline SS loadings & 0.60 & & \\
eigenvalue & & 1.18 & 1.45 \\
explained variance & 0.10 & 0.23 & 0.37 \\
\hline KMO & 0.45 & 0.45 & 0.55 \\
\hline Bartlett's test & $\mathrm{X}^{2}=19.51$ & $\mathrm{X}^{2}=18.95$ & $\mathrm{X}^{2}=19.95$ \\
& $\mathrm{df}=15$ & $\mathrm{df}=15$ & $\mathrm{df}=15$ \\
& $\mathrm{p}=0.192$ & $\mathrm{p}=0.216$ & $\mathrm{p}=0.174$ \\
\hline
\end{tabular}


Table S2-16 General intelligence including all cognitive performance measures. Interspecific G loadings of the PAF, as well as intraspecific g loadings for gray mouse lemurs (GML) and Madame Berthe's mouse lemurs (MBML). For each factor and component, the squared factor (SS) loadings or eigenvalue, and the proportion of explained variance are given. For each analysis, the sample size (N), the Kaiser-Meyer-Olkin (KMO) index, and the statistics for the Bartlett's test of sphericity are given

\begin{tabular}{|l|ccc|}
\hline cognitive performance & $\begin{array}{c}\text { G loadings } \\
\left(\mathbf{N G M L}_{\mathbf{~ = ~ 2 1 , ~ N M B M L ~}} \mathbf{9}\right)\end{array}$ & $\begin{array}{c}\text { g loadings } \\
\text { GML }(\mathbf{N}=\mathbf{2 1})\end{array}$ & $\begin{array}{c}\text { g loadings } \\
\text { MBML }(\mathbf{N}=\mathbf{9})\end{array}$ \\
\hline FE: success latency & 0.47 & -0.40 & 0.50 \\
FEP: persistence & 0.03 & -0.15 & -0.26 \\
CT: N incorrect trials & 0.29 & 0.06 & 0.36 \\
SP1: success latency & -0.45 & 0.17 & -0.41 \\
SP2: N incorrect trials & -0.01 & -0.43 & -0.39 \\
PM: N mean errors & 0.74 & -0.54 & 0.27 \\
Dv: N trials & -0.20 & -0.36 & 0.13 \\
Rv: N trials & -0.08 & -0.43 & -0.39 \\
\hline SS loadings & 1.11 & & \\
eigenvalue & & 1.43 & 1.67 \\
explained variance & 0.14 & 0.20 & 0.35 \\
\hline KMO & 0.49 & 0.32 & 0.21 \\
\hline Bartlett's test & $\mathrm{X}^{2}=24.71$ & $\mathrm{X}^{2}=36.60$ & $\mathrm{X}^{2}=26.26$ \\
& $\mathrm{df}=28$ & $\mathrm{df}=45$ & $\mathrm{df}=28$ \\
& $p=0.644$ & $p=0.810$ & $p=0.559$ \\
\hline
\end{tabular}


Table S2-17 Gray mouse lemurs, all statistic models. Given are test-statistics (ts), p-value and degrees of freedom (df) of the full-null-model comparison (bold), effect sizes $\left(R^{2}\right)$, as well as estimates and standard errors (SE), test-statistics and p-values of the individual predictors. M: male; visual stimuli: DF: dotted flower, SS: striped square, WC: black triangle

\begin{tabular}{|c|c|c|c|c|c|c|}
\hline model ID & $\begin{array}{l}\text { response } \\
\text { fixed factors }\end{array}$ & estimate $\pm S E$ & ts & p & df & $\mathbf{R}^{2}$ \\
\hline a) & $\begin{array}{l}\text { activity } \\
\quad \text { sex } \\
\text { season } \\
\text { BMI }\end{array}$ & $\begin{array}{l}\text { M: } 20.43 \pm 7.71 \\
\text { dry-wet: } 4.49 \pm 8.64 \\
-0.192 \pm 3.92\end{array}$ & $\begin{array}{l}3.00 \\
7.03 \\
0.27 \\
0.24\end{array}$ & $\begin{array}{l}\mathbf{0 . 0 3 4} \\
0.009 \\
0.604 \\
0.625\end{array}$ & 3 & 0.049 \\
\hline b) & $\begin{array}{l}\text { neophilia } \\
\text { sex } \\
\text { season } \\
\text { BMI } \\
\text { zero-part: sex }\end{array}$ & $\begin{array}{l}\text { M: }-0.04 \pm 0.16 \\
\text { dry-wet: }-0.10 \pm 0.17 \\
-0.157 \pm 0.08 \\
\text { F: }-083 \pm 0.35 \\
\text { M: }-1.53 \pm 0.73\end{array}$ & $\begin{array}{l}10.65 \\
-0.25 \\
-0.58 \\
-2.05 \\
-2.34 \\
-2.10\end{array}$ & $\begin{array}{l}\mathbf{0 . 0 3 1} \\
0.806 \\
0.561 \\
0.041 \\
0.019 \\
0.035\end{array}$ & 4 & 0.088 \\
\hline c) & $\begin{array}{l}\text { innovation spee } \\
\text { Surv(success la } \\
\text { sex } \\
\text { season } \\
\text { BMI } \\
\text { activity } \\
\text { neophilia }\end{array}$ & $\begin{array}{l}\text { cyl solver) } \\
\text { M: } 0.34 \pm 0.24 \\
\text { dry-wet: } 0.43 \pm 0.24 \\
0.22 \pm 0.14 \\
0.11 \pm 0.13 \\
0.02 \pm 0.12\end{array}$ & $\begin{array}{l}9.29 \\
2.04 \\
3.34 \\
2.58 \\
0.75 \\
0.04\end{array}$ & $\begin{array}{l}\mathbf{0 . 1 0 0} \\
0.153 \\
0.067 \\
0.108 \\
0.388 \\
0.847\end{array}$ & 5 & 0.110 \\
\hline d) & $\begin{array}{l}\text { innovation prof } \\
\text { N successes } \\
\text { sex } \\
\text { season } \\
\text { BMI } \\
\text { activity } \\
\text { neophilia }\end{array}$ & $\begin{array}{l}\text { isity } \\
\qquad \begin{array}{l}\text { M: } 0.03 \pm 0.10 \\
\text { dry-wet: } 0.04 \pm 0.09 \\
-0.02 \pm 0.05 \\
0.01 \pm 0.05 \\
0 \pm 0.05\end{array}\end{array}$ & $\begin{array}{l}\mathbf{0 . 4 8} \\
0.09 \\
0.20 \\
0.09 \\
0.07 \\
0.00\end{array}$ & $\begin{array}{l}\mathbf{0 . 9 9 3} \\
0.771 \\
0.654 \\
0.763 \\
0.795 \\
0.998\end{array}$ & 5 & 0.006 \\
\hline e) & $\begin{array}{l}\text { persistence } \\
\text { sex } \\
\text { season } \\
\text { BMI } \\
\text { activity } \\
\text { neophilia }\end{array}$ & $\begin{array}{l}\text { M: }-0.04 \pm 0.03 \\
\text { dry-wet: }-0.03 \pm 0.03 \\
-0.02 \pm 0.02 \\
0.05 \pm 0.02 \\
-0.01 \pm 0.01\end{array}$ & $\begin{array}{l}1.92 \\
1.91 \\
0.99 \\
1.49 \\
7.36 \\
0.13\end{array}$ & $\begin{array}{l}\mathbf{0 . 1 0 0} \\
0.171 \\
0.323 \\
0.226 \\
0.008 \\
0.721\end{array}$ & 5 & 0.054 \\
\hline f) & $\begin{array}{l}\text { visual discrimi } \\
\mathbf{N} \text { total trials } \\
\text { sex } \\
\text { BMI } \\
\mathbf{N} \text { total trials } \\
\text { sex } \\
\text { activity } \\
\mathbf{N} \text { total trials } \\
\text { sex } \\
\text { neophilia }\end{array}$ & $\begin{array}{l}\text { tion } \\
\text { M: } 0.11 \pm 0.19 \\
0.21 \pm 0.10 \\
\text { M: }-0.04 \pm 0.20 \\
0.12 \pm 0.10 \\
\text { M: } 0.00 \pm 0.20 \\
0.10 \pm 0.10\end{array}$ & $\begin{array}{l}2.34 \\
0.36 \\
4.66 \\
\mathbf{0 . 6 6} \\
0.03 \\
1.30 \\
\mathbf{0 . 4 7} \\
0.00 \\
0.93\end{array}$ & $\begin{array}{l}\mathbf{0 . 1 2 0} \\
0.552 \\
0.042 \\
\mathbf{0 . 5 2 8} \\
0.857 \\
0.267 \\
\mathbf{0 . 6 3 1} \\
0.995 \\
0.347\end{array}$ & 2 & -0.029 \\
\hline
\end{tabular}


Chapter III: Empirical study

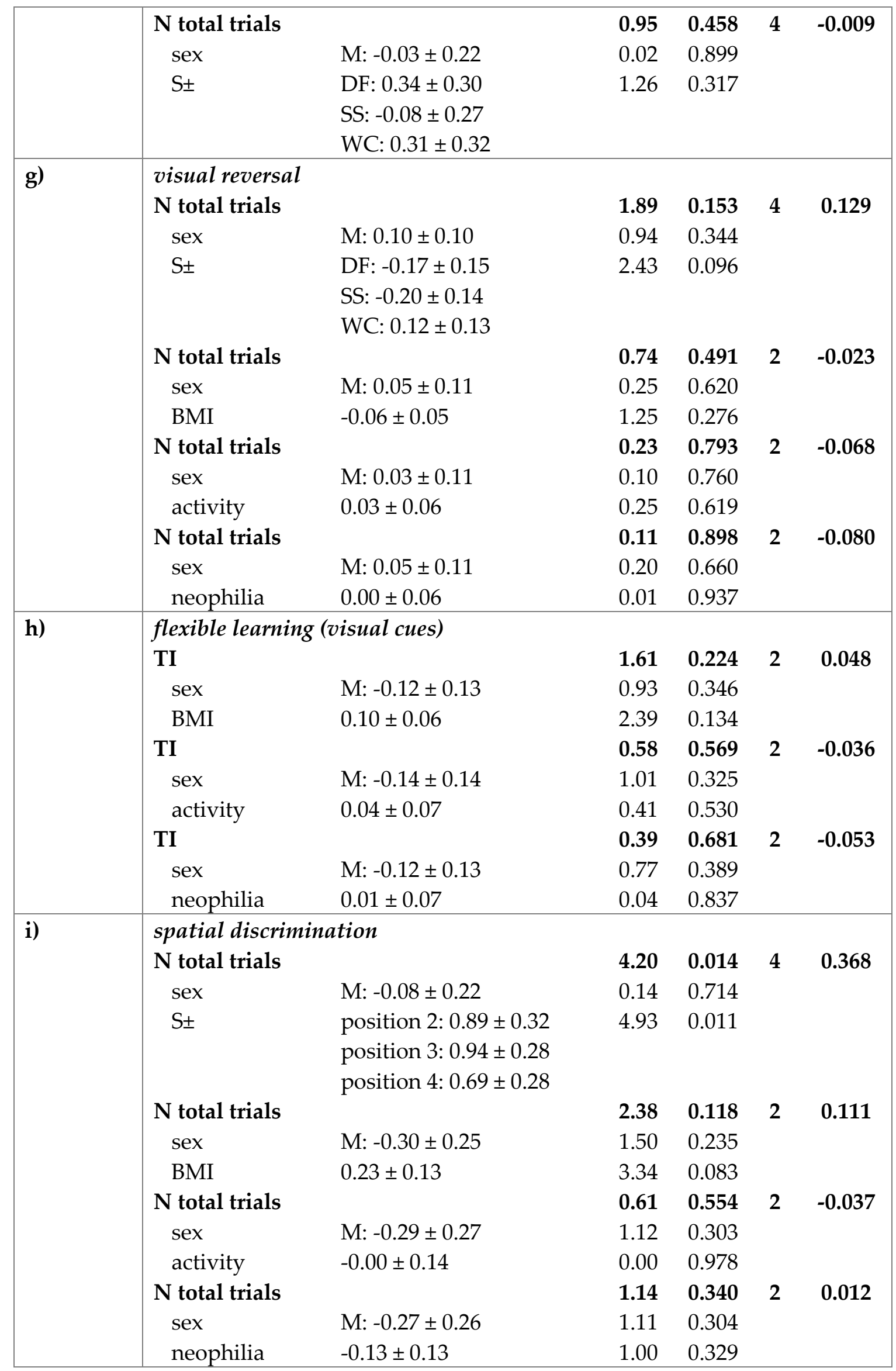




\begin{tabular}{|c|c|c|c|c|c|c|}
\hline \multirow[t]{10}{*}{ j) } & \multicolumn{6}{|c|}{ flexible learning (cue change) } \\
\hline & \multicolumn{2}{|l|}{ TI } & 4.22 & 0.029 & 2 & 0.219 \\
\hline & sex & M: $0.19 \pm 0.09$ & 4.33 & 0.049 & & \\
\hline & BMI & $-0.09 \pm 0.05$ & 4.09 & 0.056 & & \\
\hline & \multicolumn{2}{|l|}{ TI } & 2.33 & 0.122 & 2 & 0.103 \\
\hline & sex & M: $0.16 \pm 0.10$ & 2.47 & 0.131 & & \\
\hline & activity & $0.05 \pm 0.05$ & 0.85 & 0.366 & & \\
\hline & \multicolumn{2}{|l|}{ TI } & 1.90 & 0.174 & 2 & 0.073 \\
\hline & sex & M: $0.20 \pm 0.10$ & 3.79 & 0.065 & & \\
\hline & neophilia & $-0.02 \pm 0.05$ & 0.13 & 0.722 & & \\
\hline \multirow[t]{15}{*}{ k) } & \multicolumn{6}{|l|}{ spatial reversal } \\
\hline & \multicolumn{2}{|l|}{$\mathbf{N}$ total trials } & 9.65 & 0.001 & 2 & 0.464 \\
\hline & sex & M: $0.73 \pm 0.20$ & 13.75 & 0.002 & & \\
\hline & BMI & $-0.24 \pm 0.10$ & 5.90 & 0.026 & & \\
\hline & \multicolumn{2}{|l|}{$\mathbf{N}$ total trials } & 6.03 & 0.004 & 4 & 0.501 \\
\hline & sex & M: $0.66 \pm 0.20$ & 11.00 & 0.004 & & \\
\hline & \multirow[t]{3}{*}{$S \pm$} & position 2: $0.15 \pm 0.29$ & 3.23 & 0.050 & & \\
\hline & & position 3: $0.72 \pm 0.24$ & & & & \\
\hline & & position $4: 0.20 \pm 0.27$ & & & & \\
\hline & \multicolumn{2}{|l|}{$\mathbf{N}$ total trials } & 6.04 & 0.010 & 2 & 0.335 \\
\hline & $\operatorname{sex}$ & M: $0.66 \pm 0.22$ & 8.60 & 0.009 & & \\
\hline & activity & $0.13 \pm 0.11$ & 1.27 & 0.275 & & \\
\hline & $\mathbf{N}$ total trials & & 5.34 & 0.015 & 2 & 0.303 \\
\hline & sex & M: $0.70 \pm 0.22$ & 0.97 & 0.006 & & \\
\hline & neophilia & $0.07 \pm 0.12$ & 0.37 & 0.549 & & \\
\hline \multirow[t]{10}{*}{ 1) } & \multicolumn{6}{|c|}{ flexible learning (spatial cues) } \\
\hline & \multicolumn{2}{|c|}{ TI } & 4.42 & 0.016 & 3 & 0.318 \\
\hline & sex* neophilia & M: $-0.17 \pm 0.10$ & 6.17 & 0.022 & & \\
\hline & & $\begin{array}{l}\text { neophilia: }-0.20 \pm 0.06 \\
\text { neophilia:M: } 0.26 \pm 0.11\end{array}$ & & & & \\
\hline & \multicolumn{2}{|r|}{$x_{1}$} & 2.03 & 0.158 & 2 & 0.085 \\
\hline & sex & M: $-0.18 \pm 0.11$ & 2.39 & 0.138 & & \\
\hline & BMI & $0.08 \pm 0.06$ & 1.66 & 0.212 & & \\
\hline & TI & & 1.87 & 0.180 & 2 & 0.073 \\
\hline & sex & M: $-0.21 \pm 0.12$ & 3.20 & 0.089 & & \\
\hline & activity & $0.07 \pm 0.06$ & 1.38 & 0.253 & & \\
\hline \multirow[t]{8}{*}{ m) } & \multicolumn{6}{|l|}{ spatial memory } \\
\hline & \multicolumn{2}{|l|}{$\mathbf{N}$ mean errors } & 3.01 & 0.007 & 7 & 0.129 \\
\hline & sex & M: $-0.18 \pm 0.14$ & 1.64 & 0.203 & & \\
\hline & season & dry-wet: $0.06 \pm 0.14$ & 0.17 & 0.685 & & \\
\hline & BMI & $0.12 \pm 0.07$ & 3.02 & 0.086 & & \\
\hline & activity & $0.09 \pm 0.07$ & 1.64 & 0.204 & & \\
\hline & neophilia & $-0.02 \pm 0.07$ & 0.06 & 0.805 & & \\
\hline & goal box & $\begin{array}{l}\text { right: } 0.30 \pm 0.15 \\
\text { straight: }-0.44 \pm 0.20\end{array}$ & 7.22 & 0.001 & & \\
\hline
\end{tabular}




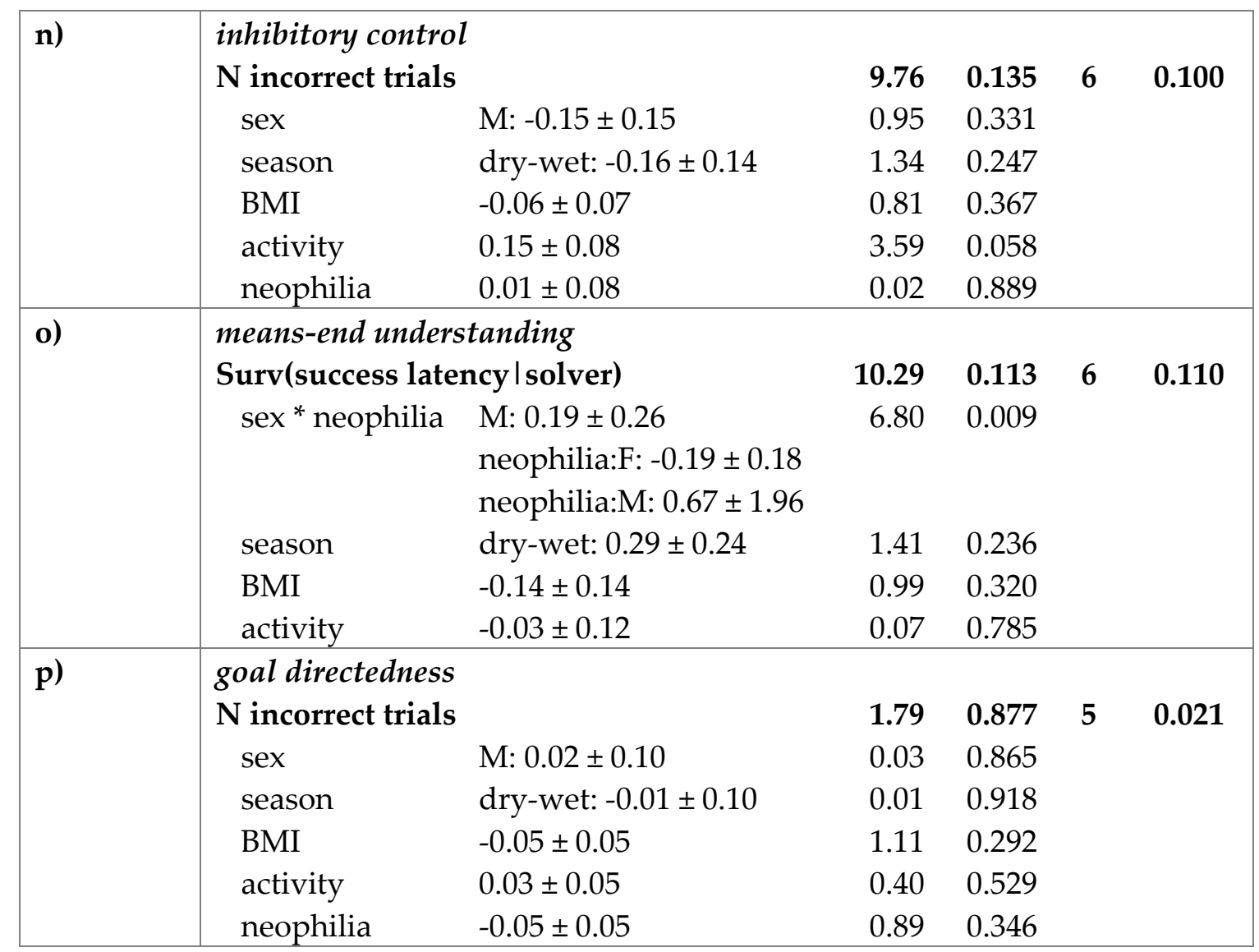

Table S2-18 Madame Berthe's mouse lemurs, all statistic models. Given are estimates, test-statistics (ts), pvalue and degrees of freedom ( $\mathrm{df}$ ) of the full-null-model comparison (bold), effect sizes $\left(\mathrm{R}^{2}\right)$, as well as estimates and standard errors (SE), test-statistics and p-values of the individual predictors. M: male; visual stimuli: DF: dotted flower, SS: striped square, WC: black triangle

\begin{tabular}{|c|c|c|c|c|c|c|}
\hline model ID & $\begin{array}{l}\text { response } \\
\text { fixed factors }\end{array}$ & estimate $\pm \mathrm{SE}$ & ts & $\mathrm{p}$ & df & $\mathbf{R}^{2}$ \\
\hline a) & $\begin{array}{l}\text { activity } \\
\text { sex } \\
\text { season } \\
\text { BMI }\end{array}$ & $\begin{array}{l}\text { M: }-13.00 \pm 15.51 \\
\text { dry-wet: }-12.23 \pm 16.83 \\
11.40 \pm 7.67\end{array}$ & $\begin{array}{l}\mathbf{1 . 2 7} \\
0.70 \\
0.53 \\
2.21\end{array}$ & $\begin{array}{l}\mathbf{0 . 3 0 3} \\
0.409 \\
0.473 \\
0.148\end{array}$ & 3 & 0.026 \\
\hline b) & $\begin{array}{l}\text { neophilia } \\
\text { sex } \\
\text { season } \\
\text { BMI } \\
\text { zero-part: sex }\end{array}$ & $\begin{array}{l}\text { M: } 0.19 \pm 0.28 \\
\text { dry-wet: }-0.06 \pm 0.31 \\
0.08 \pm 0.15 \\
\text { F: }-1.07 \pm 0.64 \\
\text { M: }-1.01 \pm 1.31\end{array}$ & $\begin{array}{r}\mathbf{1 . 7 1} \\
0.67 \\
-0.21 \\
0.55 \\
-1.68 \\
-0.77 \\
\end{array}$ & $\begin{array}{l}\mathbf{0 . 7 8 9} \\
0.501 \\
0.835 \\
0.581 \\
0.093 \\
0.439\end{array}$ & 4 & 0.053 \\
\hline c) & $\begin{array}{c}\text { innovation spec } \\
\text { Surv(success la } \\
\text { sex* activity }\end{array}$ & $\begin{array}{l}\text { solver) } \\
\text { M: } 0.52 \pm 0.43 \\
\text { activity:F: }-0.65 \pm 0.52 \\
\text { activity:M: } 1.33 \pm 0.55\end{array}$ & $\begin{array}{l}6.87 \\
6.38\end{array}$ & $\begin{array}{l}\mathbf{0 . 0 8 0} \\
0.012\end{array}$ & 3 & 0.235 \\
\hline
\end{tabular}




\begin{tabular}{|c|c|c|c|c|c|c|}
\hline & \multicolumn{2}{|c|}{ Surv(success latency| solver) } & \multirow{2}{*}{$\begin{array}{l}\mathbf{1 . 6 2} \\
1.16\end{array}$} & \multirow{2}{*}{$\begin{array}{l}\mathbf{0 . 4 0 0} \\
0.247\end{array}$} & \multirow[t]{2}{*}{2} & \multirow[t]{3}{*}{0.033} \\
\hline & sex & M: $0.52 \pm 0.45$ & & & & \\
\hline & season & dry-wet: $-0.41 \pm 0.45$ & -0.90 & 0.366 & & \\
\hline & \multicolumn{2}{|c|}{ Surv(success latency | solver) } & 0.94 & 0.600 & 2 & 0.059 \\
\hline & sex & M: $0.36 \pm 0.42$ & 0.85 & 0.396 & & \\
\hline & BMI & $-0.08 \pm 0.20$ & -0.37 & 0.713 & & \\
\hline & \multicolumn{2}{|c|}{ Surv(success latency | solver } & 1.99 & 0.400 & 2 & 0.076 \\
\hline & sex & M: $0.61 \pm 0.8$ & 1.29 & 0.200 & & \\
\hline & neophilia & $-0.30 \pm 0.27$ & -1.12 & 0.261 & & \\
\hline \multirow[t]{13}{*}{ d) } & \multicolumn{6}{|c|}{ innovation propensity } \\
\hline & \multicolumn{2}{|l|}{$\mathbf{N}$ successes } & 1.39 & 0.500 & 2 & 0.051 \\
\hline & sex & M: $0.11 \pm 0.19$ & 0.34 & 0.560 & & \\
\hline & BMI & $0.10 \pm 0.09$ & 1.18 & 0.278 & & \\
\hline & \multicolumn{2}{|l|}{ N successes } & 0.76 & 0.682 & 2 & 0.028 \\
\hline & sex & M: $0.05 \pm 0.19$ & 0.08 & 0.772 & & \\
\hline & season & dry-wet: $0.14 \pm 0.19$ & 0.56 & 0.455 & & \\
\hline & \multicolumn{2}{|l|}{$\mathbf{N}$ successes } & 1.15 & 0.562 & 2 & 0.042 \\
\hline & sex & M: $0.06 \pm 0.19$ & 0.09 & 0.759 & & \\
\hline & activity & $-0.09 \pm 0.09$ & 0.94 & 0.331 & & \\
\hline & \multicolumn{2}{|l|}{ N successes } & 0.34 & 0.844 & 2 & 0.013 \\
\hline & sex & M: $0.07 \pm 0.19$ & 0.12 & 0.725 & & \\
\hline & neophilia & $0.03 \pm 0.09$ & 0.13 & 0.717 & & \\
\hline \multirow[t]{13}{*}{ e) } & \multicolumn{2}{|l|}{ persistence } & 5.97 & 0.004 & 3 & 0.374 \\
\hline & \multirow[t]{3}{*}{ sex ${ }^{*} \mathrm{BMI}$} & M: $-0.05 \pm 0.04$ & 7.61 & 0.011 & & \\
\hline & & BMI: $-0.08 \pm 0.02$ & & & & \\
\hline & & BMI:M: $0.11 \pm 0.04$ & & & & \\
\hline & \multicolumn{2}{|l|}{ persistence } & 1.06 & 0.363 & 2 & 0.005 \\
\hline & sex & M: $-0.06 \pm 0.05$ & 1.39 & 0.250 & & \\
\hline & season & dry-wet: $0.05 \pm 0.05$ & 1.04 & 0.318 & & \\
\hline & persistence & & 1.39 & 0.270 & 2 & 0.030 \\
\hline & sex & M: $-0.06 \pm 0.05$ & 1.67 & 0.209 & & \\
\hline & activity & $-0.03 \pm 0.02$ & 1.67 & 0.210 & & \\
\hline & persistence & & 1.16 & 0.324 & 2 & 0.015 \\
\hline & sex & M: $-0.03 \pm 0.05$ & 0.54 & 0.468 & & \\
\hline & neophilia & $-0.03 \pm 0.02$ & 1.28 & 0.270 & & \\
\hline \multirow[t]{9}{*}{ f) } & \multicolumn{6}{|c|}{ visual discrimination } \\
\hline & \multicolumn{2}{|l|}{$\mathbf{N}$ total trials } & 1.02 & 0.332 & 1 & 0.002 \\
\hline & activity & $-0.08 \pm 0.08$ & 1.023 & 0.332 & & \\
\hline & \multicolumn{2}{|l|}{$\mathrm{N}$ total trials } & 0.01 & 0.927 & 1 & -0.083 \\
\hline & $\operatorname{sex}$ & M: $-0.02 \pm 0.16$ & 0.01 & 0.927 & & \\
\hline & \multicolumn{2}{|l|}{$\mathbf{N}$ total trials } & 0.59 & 0.456 & 1 & -0.032 \\
\hline & BMI & $-0.06 \pm 0.08$ & 0.59 & 0.456 & & \\
\hline & \multicolumn{2}{|l|}{$\mathrm{N}$ total trials } & 0.44 & 0.522 & 1 & -0.045 \\
\hline & neophilia & $0.05 \pm 0.08$ & 0.44 & 0.522 & & \\
\hline
\end{tabular}


Chapter III: Empirical study

\begin{tabular}{|c|c|c|c|c|c|c|}
\hline & $\begin{array}{l}\mathrm{N} \text { total trials } \\
\mathrm{S}^{+}\end{array}$ & $\begin{array}{l}\text { DF: } 0.31 \pm 0.21 \\
\text { SS: }-0.01 \pm 0.23 \\
\text { WC: } 0.04 \pm 0.23\end{array}$ & $\begin{array}{l}1.22 \\
1.22\end{array}$ & $\begin{array}{l}\mathbf{0 . 3 5 3} \\
0.353\end{array}$ & 3 & 0.048 \\
\hline \multirow[t]{4}{*}{ g) } & $\begin{array}{l}\text { visual reversal } \\
\mathrm{N} \text { total trials } \\
\mathrm{S}^{+}\end{array}$ & $\begin{array}{l}\text { DF: } 0.04 \pm 0.23 \\
\text { SS: } 0.42 \pm 0.24 \\
\text { WC: }-0.11 \pm 0.24\end{array}$ & $\begin{array}{l}2.22 \\
2.22\end{array}$ & $\begin{array}{l}\mathbf{0 . 1 6 4} \\
0.164\end{array}$ & 3 & 0.249 \\
\hline & $\begin{array}{l}\mathbf{N} \text { total trials } \\
\text { sex }\end{array}$ & M: $0.17 \pm 0.18$ & $\begin{array}{l}\mathbf{0 . 8 3} \\
0.83\end{array}$ & $\begin{array}{l}\mathbf{0 . 3 8 4} \\
0.384\end{array}$ & 1 & -0.016 \\
\hline & $\begin{array}{l}\mathbf{N} \text { total trials } \\
\text { BMI }\end{array}$ & $0.07 \pm 0.09$ & $\begin{array}{l}\mathbf{0 . 5 5} \\
0.55\end{array}$ & $\begin{array}{l}\mathbf{0 . 4 7 6} \\
0.476\end{array}$ & 1 & -0.043 \\
\hline & $\begin{array}{l}\mathbf{N} \text { total trials } \\
\text { activity } \\
\mathbf{N} \text { total trials } \\
\text { neophilia }\end{array}$ & $\begin{array}{l}-0.07 \pm 0.10 \\
-0.04 \pm 0.10\end{array}$ & $\begin{array}{l}\mathbf{0 . 5 1} \\
0.51 \\
\mathbf{0 . 1 6} \\
0.16\end{array}$ & $\begin{array}{l}\mathbf{0 . 4 9 3} \\
0.493 \\
\mathbf{0 . 7 0 0} \\
0.700\end{array}$ & 1 & $\begin{array}{l}-0.047 \\
-0.083\end{array}$ \\
\hline \multirow[t]{4}{*}{ h) } & $\begin{array}{l}\text { flexible learnin } \\
\text { TI } \\
\text { sex }\end{array}$ & $\begin{array}{l}\text { l cues) } \\
\text { M: }-0.25 \pm 0.10\end{array}$ & $\begin{array}{l}6.15 \\
6.15\end{array}$ & $\begin{array}{l}\mathbf{0 . 0 2 9} \\
0.029\end{array}$ & 1 & 0.284 \\
\hline & $\begin{array}{l}\text { TI } \\
\text { BMI }\end{array}$ & $0.05 \pm 0.06$ & $\begin{array}{l}\mathbf{0 . 5 3} \\
0.53\end{array}$ & $\begin{array}{l}\mathbf{0 . 4 8 1} \\
0.481\end{array}$ & 1 & -0.038 \\
\hline & \multicolumn{2}{|l|}{ TI } & $\begin{array}{l}\mathbf{1 . 8 5} \\
1.85\end{array}$ & $\begin{array}{l}\mathbf{0 . 1 9 8} \\
0.198\end{array}$ & 1 & 0.062 \\
\hline & \multicolumn{2}{|l|}{ TI } & $\begin{array}{l}3.75 \\
3.75\end{array}$ & $\begin{array}{l}\mathbf{0 . 0 7 7} \\
0.077\end{array}$ & 1 & 0.175 \\
\hline \multirow[t]{5}{*}{ i) } & $\begin{array}{l}\text { spatial discrim } \\
\mathbf{N} \text { total trials } \\
\text { sex }\end{array}$ & M: $-0.32 \pm 0.24$ & $\begin{array}{l}1.74 \\
1.74\end{array}$ & $\begin{array}{l}\mathbf{0 . 2 2 9} \\
0.229\end{array}$ & 1 & 0.085 \\
\hline & \multicolumn{2}{|l|}{$\mathrm{N}$ total trials } & $\begin{array}{l}\mathbf{0 . 2 1} \\
0.21\end{array}$ & $\begin{array}{l}\mathbf{0 . 6 6 1} \\
0.661\end{array}$ & 1 & -0.110 \\
\hline & \multicolumn{2}{|l|}{$\mathrm{N}$ total trials } & $\begin{array}{l}\mathbf{0 . 2 8} \\
0.28\end{array}$ & $\begin{array}{l}\mathbf{0 . 6 1 1} \\
0.611\end{array}$ & 1 & -0.098 \\
\hline & & $-0.15 \pm 0.13$ & $\begin{array}{l}\mathbf{1 . 2 7} \\
1.27\end{array}$ & $\begin{array}{l}\mathbf{0 . 2 9 8} \\
0.298\end{array}$ & 1 & 0.032 \\
\hline & $\begin{array}{l}\mathbf{N} \text { total trials } \\
\mathrm{S}^{+}\end{array}$ & $\begin{array}{l}\text { position } 2: 0.10 \pm 0.41 \\
\text { position 3: } 0.10 \pm 0.41 \\
\text { position } 4: 0.53 \pm 0.32\end{array}$ & $\begin{array}{l}\mathbf{0 . 9 4} \\
0.94\end{array}$ & $\begin{array}{l}\mathbf{0 . 4 8 9} \\
0.489\end{array}$ & 3 & -0.025 \\
\hline \multirow[t]{5}{*}{ j) } & \multicolumn{6}{|c|}{ flexible learning (cue change) } \\
\hline & $\begin{array}{l}\text { TI } \\
\text { sex }\end{array}$ & M: $0.24 \pm 0.14$ & $\begin{array}{l}2.97 \\
2.97\end{array}$ & $\begin{array}{l}\mathbf{0 . 1 1 9} \\
0.119\end{array}$ & 1 & 0.164 \\
\hline & TI & & 0.10 & 0.756 & 1 & -0.099 \\
\hline & BMI & $-0.03 \pm 0.08$ & 0.10 & 0.756 & & \\
\hline & $\begin{array}{l}\text { TI } \\
\text { activity }\end{array}$ & $-0.12 \pm 0.07$ & $\begin{array}{l}2.82 \\
2.82\end{array}$ & $\begin{array}{l}\mathbf{0 . 1 2 8} \\
0.128\end{array}$ & 1 & 0.154 \\
\hline
\end{tabular}


Chapter III: Empirical study

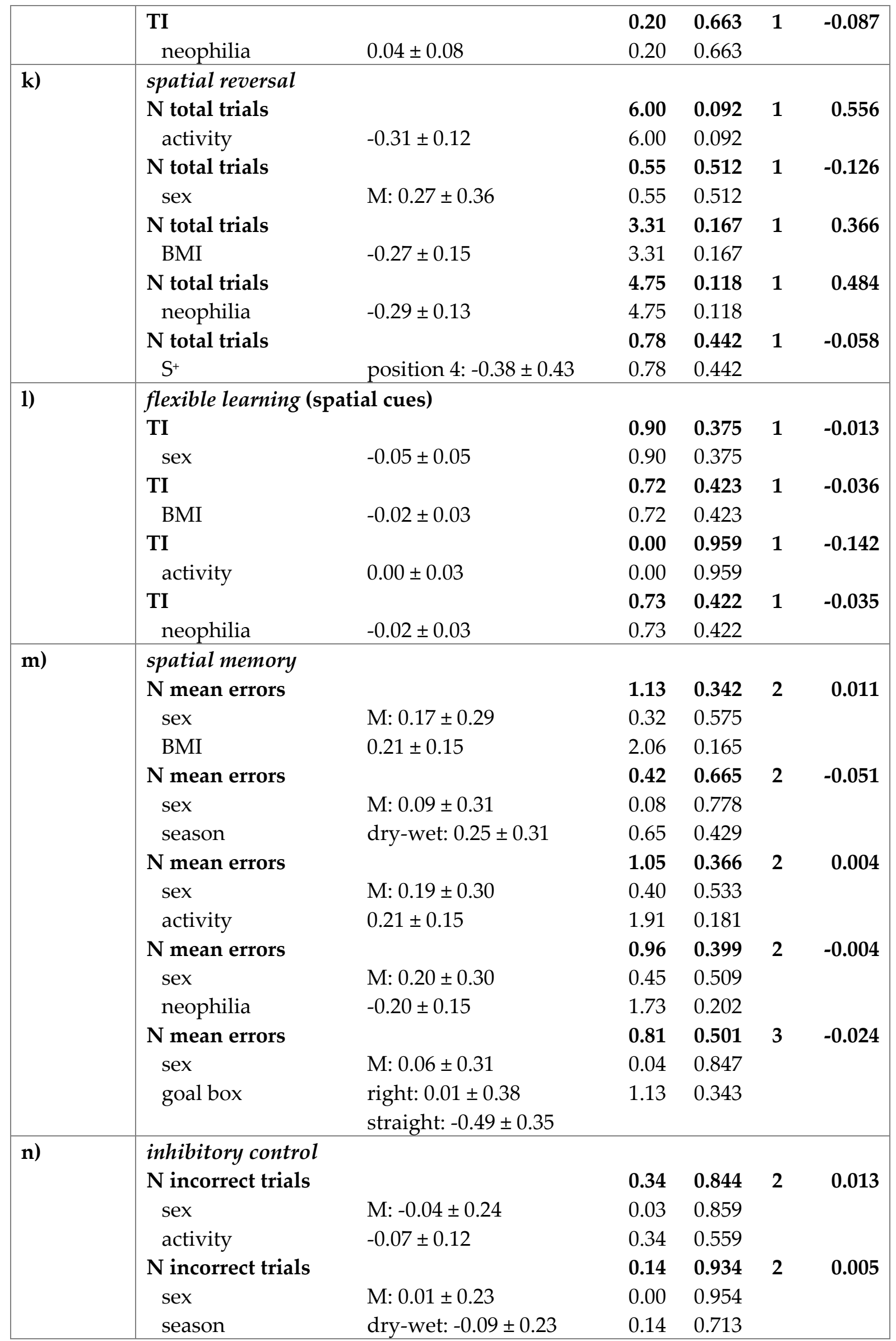


Chapter III: Empirical study

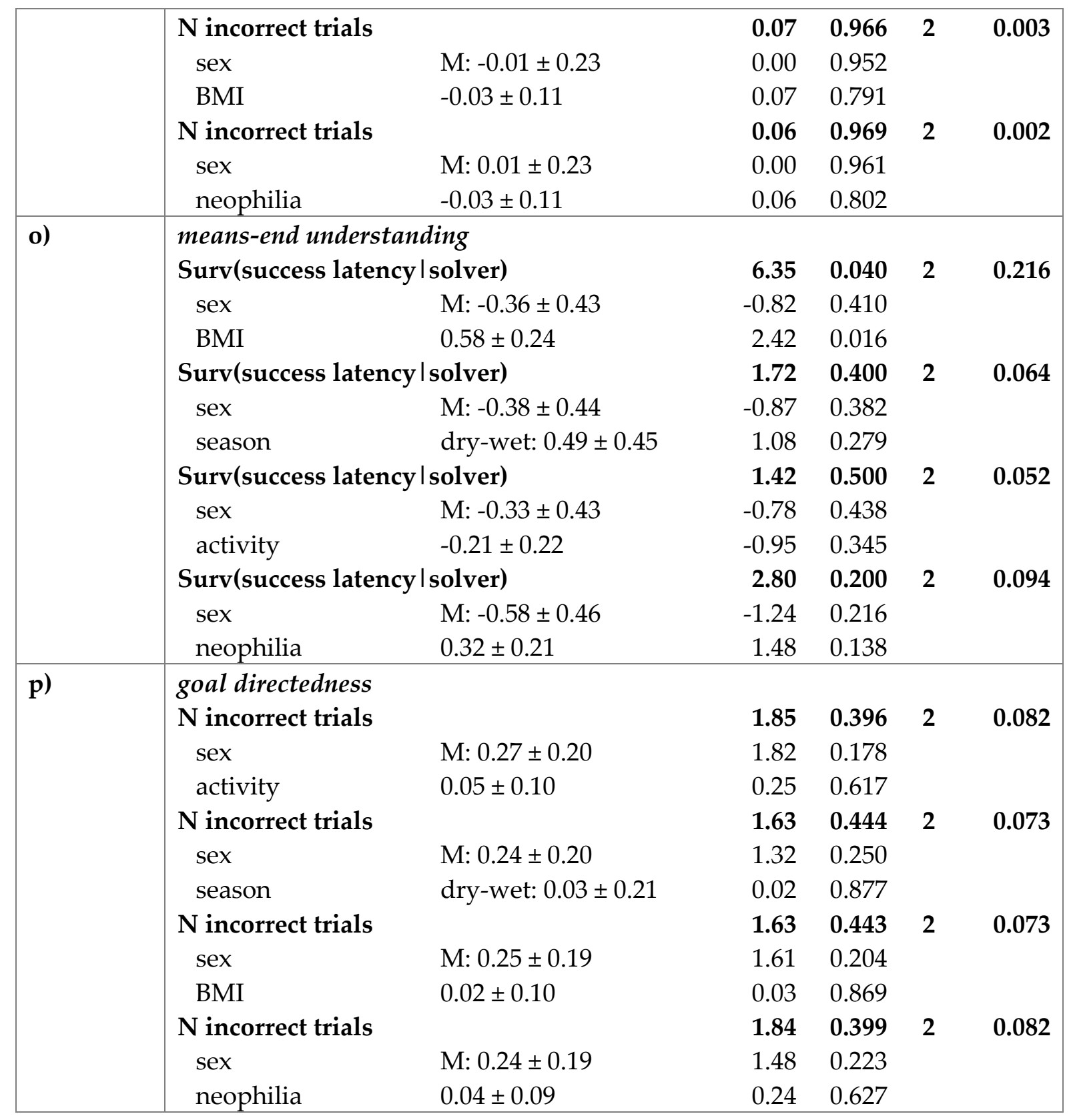




\section{Chapter IV:General discussion}

The objective of my thesis was to broaden our understanding of cognitive evolution in relation to ecological factors, with a specific focus on the degree of ecological specialization. The specific aims were to summarize common links of variation in cognitive test performances with ecological factors across the animal kingdom (Chapter II), and to link cognitive performances across a broad range of cognitive abilities with the degree of ecological specialization and species-specific ecological adaptations (Chapter III). To this end, I systematically investigated patterns of (co)variation between cognitive performances and species-specific ecological adaptations across the animal kingdom, and empirically assessed cognitive performances in two sympatric mouse lemur species, that differ in their degree of ecological specialization. In my first theoretic approach, I found that cognitive performances have been associated with ecological factors, such as food patch distribution, characteristics of food items, habitat complexity or seasonality. Other ecological factors, such as the synecology with predators or parasites remain largely understudied. Also, a covariation of cognitive performances with the degree of ecological specialization has rarely been explicitly investigated. By classifying the species in comparison in their degree of dietary and habitat specialization, I found that this categorical proxy for a species-specific ecological niche does not consistently correlate with cognitive performances (Chapter II). In my second experimental approach, I found that the generalist gray mouse lemur does not consistently outperform the more specialized and sympatric Madame Berthe's mouse lemur across a broad range of cognitive abilities. The gray mouse lemur was more innovative and showed greater persistence, whereas the Madame Berthe's mouse lemur was more active and learned associative reward contingencies faster. The two species did not differ in inhibitory control, means-end understanding, or goal directedness (Chapter III). These experimental results parallel the theoretic findings of the reviewed literature, in that generalist species do not outperform specialist species per se. Instead, generalists are specifically more innovative, which may provide them the adaptive advantage to better cope with rapidly changing environmental conditions. Other cognitive abilities, however, may rather covary with other ecological adaptations, such as species-specific ranging behavior or foraging 
strategies, or social factors that are unrelated to the degree of dietary and habitat specialization.

In this last chapter, I evaluate the compiled results by integrating them into the theory of cognitive variation and evolutionary development. Additionally, I discuss how my results can be useful for future studies on cognition within the animal kingdom.

\section{The generalist's advantage?}

Studies examining innovative problem-solving, reversal leaning abilities or inhibitory control, aim to investigate an individual's behavioral flexibility (Amici et al. 2008; Griffin and Guez 2014; Beran 2015; Griffin 2016). Having the potential to behave flexibly, allows an individual to better adapt to variable conditions (Lee 2003; Lefebvre et al. 2004; Sol et al. 2005a; Lea et al. 2020). Generalists are exposed to more variable conditions, i.e., a greater range of habitat types or a broader dietary niche. Thus, they presumably face a greater variety of ecological challenges in their natural habitats, which might explain their advantageous ability to invent novel or modified solutions to occurring problems (Reader and Laland 2003; Sol et al. 2005a). In fact, large literature reviews on innovation counts report generalist birds and primates to be more innovative than more specialized species (Overington et al. 2011; Ducatez et al. 2015). Similarly, dietary generalist primates possess better inhibitory control than more specialized primates (MacLean et al. 2014). Additionally, most of the here reviewed experimental studies reported generalists to outperform more specialized species in innovative problem-solving or reversal learning tasks (Day et al. 1999a; Day et al. 2003; Bond et al. 2007; Griffin and Diquelou 2015; Szabo and Whiting 2020). In mouse lemurs, I could replicate the correlation regarding innovative potential, with the generalist gray mouse lemurs performing better in the innovative

problem-solving task. However, the more specialized Madame Berthe's mouse lemur learned reversed reward contingencies faster, while the two species only partly differed in their degree of an initial flexible response (transfer index) and did not differ in their inhibitory control. Additionally, innovative propensity and inhibitory control did not correlate with each other, with the transfer indices or the discrimination and reversal 
learning performances. Hence, these proxies may capture different aspects of flexibility in mouse lemurs.

In a previous study comparing innovative abilities in the same species, the more specialized Madame Berthe's mouse lemur outperformed the generalist gray mouse lemur in a modified version of the presented problem-solving task (Henke-von der Malsburg and Fichtel 2018). In that study, gray mouse lemurs persisted to use a previously successful solving technique, while Madame Berthe's mouse lemurs were less conservative and, thus, better able to adjust their behavior to a more efficient solution (Henke-von der Malsburg and Fichtel 2018). Persistence reflects task-directed motivation and seems to be specifically important for innovative problem-solving using novel extractive foraging tasks (Benson-Amram and Holekamp 2012; Thornton and Samson 2012; Huebner and Fichtel 2015; Chow et al. 2016; Guez and Griffin 2016). However, persistence can hinder efficient problem-solving, when individuals conservatively use previous solving techniques which result in a high number of perseverative errors (Hauser 1999). In general, conservatism may prevent generalists to encounter potentially dangerous situations, such as the production of more costly and/or less efficient novel or modified behaviors (Omnivore's paradox; Rozin 1976). This conservatism can be specifically favored over a more flexible response, when animals face more complex modifications (Chow et al. 2016; Davis et al. 2019; Lea et al. 2020), as it may have been the case in the previous study (Henke-von der Malsburg and Fichtel 2018) and in the here presented reversal learning paradigm.

This challenges the notion of the generalist's advantage to respond flexibly to changing environmental conditions, which, amongst others, come along with the current climatic change or anthropogenic disturbances (Sol et al. 2002; Schuck-Paim et al. 2008; Wilson and Yoshimura 2008; Wright et al. 2010).

\subsection{Insufficient explanatory potential of the categorical system of niche theory}

Despite the apparent superposition of generalists with respect to innovative problemsolving, they do not consistently outperform specialists in other cognitive tasks. Thus, 
categorizing species into generalists and specialists cannot sufficiently explain speciesspecific cognitive adaptations. As with any categorical system, the degree of ecological specialization captures only a subset of an animal's ecological adaptations (Hutchinson 1957; Devictor et al. 2010). Moreover, an animal can be a generalist regarding one niche dimension, while being more specialized within another dimension (Futuyma and Moreno 1988). For example, golden lion tamarins (Leontopithecus rosalia) are omnivorous, while being restricted to the tropical rain forest in south-eastern Brazil (Day et al. 2003). They are, thus, categorized as dietary generalists and habitat specialists. Additionally, the classification can either be based on the relative comparison to other species, or on an estimate, such as Levin's standardized index which measures dietary breadth (Krebs 1989). For instance, the fat-tailed dwarf lemur (Cheirogaleus medius) is broadly described as omnivorous, but prefers a frugivorous diet, which leads to a more specialized classification (Lahann 2007; Teichroeb and Vining 2019). Similarly, the gray mouse lemur has a broad dietary breadth (Levin's standardized index $B=0.63$ ) and adjusts its diet flexibly to seasonal food availabilities throughout the year in dry-deciduous forests. However, in the same area, at the onset of the dry season they feed on a large proportion on homopteran secretions $(>60 \%)$ which is mostly complemented with animal matter (Dammhahn and Kappeler 2008a; Dammhahn and Kappeler 2008b). Whereas in rain forests, they are mainly frugivorous year-round (Atsalis 1999; Lahann 2007). Thus, regarding its dietary preferences and depending on season and region, the gray mouse lemur maybe categorized as generalist or as more specialized when their mean dietary breadth throughout the year is not estimated using a metric index.

While providing a ubiquitous scaling system, niche breadth estimates are rarely used to classify a species ecological niche in cognitive studies. These studies rather provide a descriptive overview of a species' preferences which then are correlated with cognitive performances (Henry and Stoner 2011; Teichroeb and Vining 2019). Additionally, studies systematically estimating a species ecological niche, are only available for a subset of species, making it difficult to compare interspecific variation across studies. Similarly, a numeric degree of ecological specialization, which should categorize a certain ecological niche (Hutchinson 1953), is sparsely available across species and can be differently defined and measured. When defining the ecological specialization as niche breadth, the diversity 
of resources a species uses would be measured (Roughgarden 1972; Bolnick et al. 2003). Accordingly, a specialist would use a narrower niche than a generalist. Alternatively, the ecological specialization can be defined as niche overlap. Here, the deviation of used resources in comparison to another species would be measured and a species would be categorized as specialist if it uses resources which are only rarely used by other species (Bolnick et al. 2002). Consequently, the interspecific comparison of cognitive abilities in relation to the species' degree of ecological specialization can be already confounded by methodological issues.

\subsection{Factors explaining interspecific variation in cognitive abilities}

\subsubsection{Social and cultural intelligence}

Social factors can covary with both, social and non-social cognition (Whiten and van Schaik 2007). For instance, social group size correlated positively with performances in reversal learning paradigms in birds on the species and the individual level (Bond et al. 2007; Ashton et al. 2018a; Ashton et al. 2019). Social group size and dominance hierarchies, as well as dynamics in fission-fusion systems correlated positively with performances on transitive inference and inhibitory control in primates and birds (MacLean et al. 2008; Amici et al. 2008; Miller et al. 2019).

Consequently, supporters of the Social Intelligence Hypothesis (Humphrey 1976; Whiten and Byrne 1988) or the related Cultural Intelligence Hypothesis (Whiten and van Schaik 2007; Herrmann et al. 2007; Street et al. 2017) suggest that social complexity broadly covaries with various cognitive abilities, not only within the social domain. These ideas are based on human evolution where humans have evolved outstanding cognitive abilities, specifically in western, educated, industrialized, rich, and democratic (WEIRD) communities. The formation of cultural structures thereby seems to evoke an immense selection pressure towards better cognitive abilities and ultimately to enlarged brains (Jerison 1973; Harvey et al. 1980; Herrmann et al. 2007; Street et al. 2017). Cultural structures are considered to include a sophisticated vocal communication, and physical cognition that is transmitted and spread via forms of social learning (Galef 1992; Boyd and Richerson 1996; De Waal 1999). Traits that are also apparent in non-human animals. 
Consequently, recent research investigated cultural formation also in non-human primates, birds, or cetaceans that live in well-organized social groups, communicate vocally (e.g., bird songs), possess well-developed physical cognition (e.g., tool-use), as well as forms of social learning (e.g., motor imitation) (Whiten 2000; Rendell and Whitehead 2001; Whiten 2017; Aplin 2019). The Cultural Intelligence Hypothesis found, for instance, support in non-human primates, when great apes were reported to possess better cognitive abilities than monkeys and prosimians in terms of tool-use (Kittler et al. 2018), flexible learning (Rumbaugh and Gill 1973), or social learning (Reader and Laland 2002).

However, the great ape's superposition is not consistent across studies and cognitive traits (Deaner et al. 2006; Herrmann et al. 2007; Reader et al. 2011; Herrmann and Call 2012; Amici et al. 2012), contradicting the General Intelligence Hypothesis, which posits the convergent evolution of cognitive abilities within and across cognitive domains (Spearman 1904; Humphreys 1979). Instead, cognitive abilities may evolve in a rather domain-specific way, which is supported by the mixed evidence for general intelligence in other non-human animals (Cosmides and Tooby 1994; reviewed in Burkart et al. 2017), including mouse lemurs, both, on the species-level and on the individual-level (Kittler 2017; Huebner 2020). Hence, different cognitive abilities may be differently selected for, leading to a more modular representation of cognitive abilities (Amici et al. 2012; DeCasien and Higham 2019).

\subsubsection{Ecological intelligence}

Cognitive abilities can covary with ecological factors when they provide benefits for daily challenges, such as efficient habitat exploration and exploitation (Dukas 1998; Dukas 2004). In this respect, the assessment of spatial abilities receives great scientific attention, as spatial cognitive traits can be advantageous for solving various ecological problems, such as to efficiently navigate through large home ranges or complex habitats, or to (re-)locate previous, stationary or distributed food patches. Thus, spatial abilities covary broadly with a species diet (e.g., frugivory) or a migratory lifestyle (Platt et al. 1996; Pleskacheva et al. 2000; Cristol et al. 2003; Barkley and Jacobs 2007; Bond et al. 2007; Haupt 
et al. 2010; Henry and Stoner 2011; Rosati and Hare 2012; Clarin et al. 2013; Rosati et al. 2014; White and Brown 2015a; Trapanese et al. 2019; Teichroeb and Vining 2019).

Moreover, in most of the reviewed studies investigating variation in spatial abilities, the more generalist species outperformed the more specialized species. This parallels the experimental finding in mouse lemurs, with the gray mouse lemur achieving higher performance scores than the Madame Berthe's mouse lemur in the plus maze. However, this result was unexpected, since Madame Berthe's mouse lemurs have larger home ranges and rely more on stationary food resources (Dammhahn and Kappeler 2005; Dammhahn and Kappeler 2008b) for which they would benefit from enhanced spatial abilities. However, their spatial performance may be confounded by individual characteristics, or methodological factors, that I discuss below.

Another frequently examined ecological factor covarying with cognitive variation, is a species' diet and related foraging behaviors. According to its diet, a species has evolved specific foraging strategies that can involve certain cognitive abilities. For instance, the dependence on scatter hoarding or food-caching requires spatial memory abilities to relocate the cached food items (Clayton and Krebs 1994a). Consequently, species that are more dependent on these foraging strategies, perform better in spatial memory tasks (Cristol et al. 2003; Barkley and Jacobs 2007; Bond et al. 2007). However, food-caching Clark's nutcrackers performed worse than non-caching Mexican jays in a spatial memory task, where they were supposed to recover food caches made by conspecifics (Bednekoff and Balda 1996). In that study, the unexpected species difference with regard to a correlation with ecological adaptations, was explained by social factors, since Mexican jays experience more complex social organizations than Clark's nutcrackers (Bednekoff and Balda 1996). Thus, potential interactions between ecological and social factors must be considered when designing experimental tasks.

Species feeding predominantly on embedded or cryptic food items, benefit from cognitive abilities that facilitate the detection and extraction of these food items (Parker and Gibson 1977; Vitale et al. 1990). For instance, in order to extract concealed prey, lion tamarins (Leonthopitecus spp.) have not only evolved specialized morphological structures, i.e., elongated fingers, but also extractive manipulative techniques (Dietz et al. 1997). In 
comparison to other callitrichids, that are less extractive or visual foraging, they, subsequently perform better in extractive problem-solving tasks (Day et al. 2003).

Additionally, discriminative abilities allow to detect and discriminate between food items with higher nutritional value from food items with lower nutritional value or even detrimental food items which becomes specifically important for species feeding on ephemeral or cryptic prey (Shettleworth 2001; Dukas 2004). However, in my literature survey I did not detect comparative studies that investigated discriminative abilities between species within the same order that differed in this specific foraging ecology. Nevertheless, when primates that do not choose between ephemeral and resident food items on a regular basis, were compared to cleaner fish that prefer to clean and feed on ephemeral clients, cleaner fish performed better in a respective dichotomous choice task (Salwiczek et al. 2012; Prétôt et al. 2016). However, given the great phylogenetic distance between fish and primates, the different task performance might also result from other factors, such as adjusted experimental designs or species-specific solving strategies.

In mouse lemurs, I found Madame Berthe's mouse lemurs to learn visual and spatial reward associations faster than gray mouse lemurs. However, Madame Berthe's mouse lemurs feed predominantly on homopteran secretions (Dammhahn and Kappeler 2008b) which does not imply an apparent need for specific discrimination abilities. Thus, the interspecific variation in discrimination learning performance might be explained by other factors that do not necessarily relate to their foraging ecology. Specifically, as the presented artificial stimuli did not relate to any naturalistic stimulus mouse lemurs encounter in their natural habitat.

In addition to dietary or habitat characteristics, ecological factors related to a synecology with predators or parasites might also covary with certain cognitive abilities. However, factors related to a species-specific synecology have rarely been associated with cognitive abilities. One example is provided by an intraspecific comparison in guppies (Poecilia reticulata), where predation risk was related to spatial memory performance (Burns and Rodd 2008). When compared to the population in the low-predation regime, the population in the high-predation regime was described as less hasty and tended to have larger telencephalons, which would provide greater cognitive capacity to process spatial information. Yet, the two populations did not differ in their spatial memory 
performance (Burns and Rodd 2008). Concerning a potential link between cognitive performance and parasitism, a conclusive review on birds suggests that intraspecific variation in cognition covaries with exposure to parasites, as well as metabolic and energetic influences by the parasite on the host (Ducatez et al. 2020a). However, respective patterns seem to be mixed across species and do not yet allow for general conclusions. Respective interspecific comparisons have rarely been conducted, but suggest a positive correlation between innovation rates and parasite load in birds (Garamszegi et al. 2007; Vas et al. 2011; Soler et al. 2012).

In summary, cognitive abilities in non-human animals coevolve with social and ecological factors, but not consistently with the degree of ecological specialization. While species-specific foraging behavior, habitat complexity, or responses to seasonal variability have been linked with innovative propensities, flexible problem-solving performances, or spatial memory abilities, potential patterns of covariation with the predator regime or parasite abundances require further systematic investigations.

\section{Coevolution with brain size and fitness implications}

Explaining variation in cognitive abilities with socio-ecological factors constitutes one step towards an understanding of how cognition evolves. However, in order to fully understand the evolution of cognition, we need to elucidate the benefits of cognitive abilities that are genetically inherited and ultimately lead to a greater fitness (Dukas 2004; Thornton and Lukas 2012; Thornton et al. 2014).

\subsection{Linking cognition with brain size proxies}

The fundamental structural variation of the brain underlies a cost-benefit trade-off with energetic requirements, and it is phylogenetically constrained (Laughlin et al. 1998; Lennie 2003; Kotrschal et al. 2013; Logan et al. 2018). Various proxies of cortical development have been linked to cognitive abilities (Jerison 1973; Healy and Rowe 2007; Kotrschal et al. 2013; Logan et al. 2018). Such proxies can be measures of absolute or relative brain size, as well as assessments of cortical development by determining neuron density or connectivity or 
by ascertaining volume or density of gray and white matter. Which proxy is subsequently used to explain cognitive abilities, differs across studies and, more importantly, affects patterns of covariation (Deaner et al. 2000; Deaner et al. 2007; Healy and Rowe 2007). Additionally, data acquisition on brain structures can be complicated or even impossible in some species due to methodological or ethical issues. It is, therefore, difficult to reliably relate cognitive performance measures with structural variations of the brain across a broad range of species within the animal kingdom.

In the scope of my thesis, I did not find a consistent covariation of cognitive abilities with cortical measures, which were mostly extracted from secondary literature (Chapter II). In the subjected wild mouse lemurs (Chapter III), it was ethically not justifiable to euthanize single individuals with the purpose to measure brain sizes. Instead, a rough estimation of head volumes based on head width, indicates that gray mouse lemurs have, on average, slightly larger absolute and relative brain sizes than Madame Berthe's mouse lemurs (absolute: gray mouse lemur: $4.88 \pm 0.96 \mathrm{~cm}^{3}$, Madame Berthe's mouse lemur: $3.50 \pm 1.12 \mathrm{~cm}^{3}$; relative: gray mouse lemur: $55.45 \pm 9.53 \mathrm{~mm}^{3}$, Madame Berthe's mouse lemur: $47.14 \pm 12.63 \mathrm{~mm}^{3}$; Appendix 3, Table S3-1). Additionally, systematic prior investigations reported that dietary generalist birds and primates developed larger brains than dietary specialist species (Reader 2003; Lefebvre et al. 2004; Sol et al. 2005b; Ducatez et al. 2015). Thus, if brain size was a reliable predictor for cognitive abilities, gray mouse lemurs should consistently outperform Madame Berthe's mouse lemurs. Since that was not the case, I cannot conclude that the size of the whole brain covaries with all cognitive abilities in mouse lemurs. Instead, brain size may correlate with innovative propensities, as suggested by previous studies (Reader and Laland 2002; Ratcliffe et al. 2006; Sayol et al. 2016; Buechel et al. 2018; Ducatez et al. 2020b), while other cognitive abilities may better correlate with the size or structure of single brain areas that I could not estimate in wild mouse lemurs.

\subsection{Adaptive advantage of enhanced cognitive abilities}

Developing a larger brain and/or enhanced cognitive abilities can have fitness implications. This relationship is important as it builds the basis for natural selection on 
heritable variations. Investigating effects of cognitive variation on individual fitness can, thus, elucidate the adaptive value of cognitive abilities, as well as of cortical development (Thornton et al. 2014). For example, innovative propensities relate with greater invasion success and a greater diversification potential (Sol and Lefebvre 2000; Sol et al. 2002; Nicolakakis et al. 2003; Sol et al. 2005c; Griffin and Diquelou 2015; Reader et al. 2016). More accurate spatial memory or problem-solving performance were linked with male mating success or reproductive success in birds (Keagy et al. 2009; Cole et al. 2012; Cauchard et al. 2017; Wetzel 2017; Shaw et al. 2019; Sonnenberg et al. 2019). Faster responses to predation stimuli or better spatial memory increased survival in rodents (Maille and Schradin 2016b). However, survival was not linked to spatial memory or problem-solving performance in gray mouse lemurs (Huebner et al. 2018). Thus, enhanced cognitive abilities may provide adaptive fitness advantages. However, this research field has not yet received great empirical interest and needs further exploration to allow broad and general conclusions across the animal kingdom.

Overall, cognitive abilities evolve in covariation with social and ecological factors, can covary with cortical development and can provide fitness benefits (Thornton et al. 2014). However, cortical development also has fitness costs, due to a high energy consumption (Laughlin et al. 1998; Lennie 2003; Kotrschal et al. 2013). Additionally, as social and ecological factors are likely to interact with each other, it is difficult to disentangle their exclusive impact on the evolution of cognition. Similarly, occupying different ecological niches, while experiencing similar ecological challenges, assumes complex evolutionary processes for ecological adaptations that require systematic and more in-depth future investigations.

\section{Limitations and future implications}

With the present study, I provide a broad overview of how cognitive abilities can vary with species-specific ecological adaptations. By investigating patterns of covariation between cognitive task performances and the degree of ecological specialization, I broaden our understanding of how cognitive abilities link with a species ecological niche. However, this niche concept is only a categorical estimation of species-specific ecological 
adaptations. To further understand to which extent certain ecological factors relate with certain cognitive abilities, future studies might profit from focusing on metric ecological variables. Specifically, niche breadth can be assessed by behavioral observations in combination with fecal analyses or environmental characteristics, resulting in a metric standardized index (Colwell and Futuyma 1971; Krebs 1989), which could be related to cognitive performance measures.

Further, my thesis stresses the importance to consider several methodological aspects of testing animals, especially in the wild. Experimental approaches must be properly designed to match a species' morphological possibilities, but also to reduce potential internal (e.g., motivational aspects, personality traits) or external influences (e.g., distractions by other organisms, distracting interactions with human experimenters) to a minimum, or to be able to adequately control for such potential influences (Barrett 2014; Rowe and Healy 2014; Thornton 2014; Schubiger et al. 2020). First, considering the here conducted plus maze, the spatial learning score was confounded by species-specific avoidance behavior. Although neophilia scores did not correlate with spatial learning scores, it is likely that other individual characteristics, such as boldness impacted individual's behaviors in this relatively open arena. Indeed, bolder individuals explore their environment more actively which can influence learning performances (Sih et al. 2004; Verdolin and Harper 2013; Guenther et al. 2014; Liedtke and Fromhage 2019). It is therefore important to consider and control for various non-cognitive traits (Thornton and Lukas 2012; Griffin and Guez 2014; Griffin et al. 2015; Dougherty et al. 2018).

Second, automatic testing procedures reduce distracting interactions with the human experimenter and facilitate the execution of cognitive tasks that require multiple trials (Wild et al. 2008; Fagot and Bonté 2010; McBride et al. 2016; Wither et al. 2020). However, to date most available automatic testing apparatuses are not yet well adapted to field studies, as they require stable set-ups and constant energy supply, and cannot sustain high temperatures, humidity, or relatively dusty environments.

Third, in experimental tasks, researcher usually use stimuli that might not be relevant in the natural environment of an animal (Lahti 2015). On purpose, rather artificial stimuli are chosen with the aim to solely measure specific behavioral traits instead of a behavioral response towards a potentially familiar stimulus that would influence the 
measured performance score by experience (Rowe and Healy 2014; Shaw and Schmelz 2017). However, when these artificial stimuli do not have any relevance for the study subject, there might simply be no need for the animal to learn an association or to explore the presented stimulus/task, resulting in relatively low performances and rather arbitrary patterns of variation that do not match the species-specific ecological relevance (Rowe and Healy 2014; Gingins and Bshary 2016; van Horik and Madden 2016; Farashahi et al. 2020). Additionally, a defined "better" cognitive performance depends on the researchers understanding of how a given task is designed to be solved. However, the subjected animal may perceive the presented problem differently and approach the task in a different manner than expected. This can result in "lower" performance scores which do not necessarily reflect the actual cognitive ability (Byrne and Bates 2006; Bayne et al. 2019).

Finally, despite increasing efforts, cognitive testing and the use of comprehensive test batteries remain rare in wild animals (Halsey et al. 2006; Benson-Amram and Holekamp 2012; Thornton and Samson 2012; Shaw et al. 2015; Cauchoix et al. 2017; Huebner et al. 2018; Audet et al. 2018; Henke-von der Malsburg and Fichtel 2018; Kumpan et al. 2020). However, to better understand, which ecological factors covary with cognitive performances and to disentangle social and ecological influences on cognitive variation, we need to test individuals in their natural ecological environment rather than under controlled conditions in captivity and to compare sympatric species differing in their socio-ecological adaptations (Domjan and Galef 1983; Pritchard et al. 2016). The thereby accumulated information could further impact future conservation efforts (Greggor et al. 2014; Ratsimbazafy et al. 2016; Dinnen and Ware 2020), which would be desirable for many endangered species (Schwitzer et al. 2014b; Schwitzer et al. 2014a; Estrada et al. 2017).

\section{Conclusions}

As the first large scale comparative approach to assess cognitive variation among species differing in their degree of ecological specialization, the results of my thesis offer substantial insight into the link between cognition and ecological factors, as well as the evolution of cognitive abilities on the species-level. The results of my literature review and 
my empirical cognitive test battery show, that generalist species do not outperform more specialized species per se. Nevertheless, their greater innovative potential may allow for more adaptive responses towards changing environmental conditions. Other cognitive abilities, however, can be better explained by species-specific foraging or ranging behavior that do not necessarily correlate with the degree of ecological specialization. Additionally, cognitive abilities may rather evolve in a domain-specific way, as I did not find evidence for a general intelligence factor in mouse lemurs which parallels other studies on nonhuman animals. Essentially, cognitive performances covary with a complex of several ecological and social factors, as well as individual characteristics. To disentangle single relationships, it is advisable to investigate patterns of covariation between different cognitive performances and measurable socio-ecological factors that go beyond speciesspecific foraging behavior or social group size, and to include various species across the animal kingdom. 


\section{Appendix 3}

Table S3-1 Head volumes for a total of 422 gray mouse lemurs ( $\mathrm{N}=145$ females, $\mathrm{N}=277$ males; Mmu) and 42 Madame Berthe's mouse lemurs ( $\mathrm{N}=23$ females, $\mathrm{N}=19$ males; Mbe) that were captured between April 2013 and November 2019. The total data set of 865 measures for Mmu and 106 measures for Mbe includes repeated measures (mean $=3.04 \pm 1.57$ times) for $223 \mathrm{Mmu}$ and $26 \mathrm{Mbe}$. Absolute head volumes were estimated using the formula $4 / 3 \pi \mathrm{r}^{\wedge} 3$, with $\mathrm{r}$ being half of the head width [mm]. Relative head volumes were related to the individuals' body length [mm]. Given are mean \pm standard deviation (SD), minimum and maximum values

\begin{tabular}{|ll|lll|lll|}
\hline \multirow{2}{*}{ species } & \multirow{2}{*}{ sex } & \multicolumn{3}{|c|}{ absolute $\left[\mathrm{cm}^{3}\right]$} & \multicolumn{3}{c|}{ relative $\left[\mathrm{mm}^{3}\right]$} \\
& & mean \pm SD & min & max & mean \pm SD & min & max \\
\hline Mmu & combined & $4.88 \pm 0.96$ & 2.57 & 18.48 & $55.45 \pm 9.53$ & 8.94 & 205.30 \\
& female & $4.97 \pm 1.27$ & 2.57 & 18.48 & $55.70 \pm 12.81$ & 8.94 & 205.30 \\
& male & $4.83 \pm 0.70$ & 3.11 & 14.00 & $55.30 \pm 6.82$ & 38.82 & 147.33 \\
\hline Mbe & combined & $3.50 \pm 1.12$ & 2.15 & 14.00 & $47.14 \pm 12.63$ & 30.62 & 164.66 \\
& female & $3.65 \pm 1.42$ & 2.31 & 14.00 & $48.14 \pm 16.01$ & 34.57 & 164.66 \\
& male & $3.28 \pm 0.36$ & 2.15 & 4.00 & $45.76 \pm 4.99$ & 30.62 & 53.37 \\
\hline
\end{tabular}




\section{References}

Aicha M, Hadjer A, Raounak K, Karima M (2017) Diet analysis of Acanthodactylus scutellatus (AUDOUIN, 1809) (Lacertidae) in the Algerian septentrional Sahara (Oued Souf). J Entomol Zool Stud 5:302-305

Alvarez J, Willig MR, Jones KJ, Webster DW (1991) Glossophaga soricina. Am Soc Mammal 379:1-7

Amici F, Aureli F, Call J (2008) Fission-fusion dynamics, behavioral flexibility, and inhibitory control in primates. Curr Biol 18:1415-1419 . https://doi.org/10.1016/j.cub.2008.08.020

Amici F, Barney B, Johnson VE, Call J, Aureli F (2012) A modular mind? A test using individual data from seven primate species. PLoS ONE 7:e51918. https://doi.org/10.1371/journal.pone.0051918

Amici F, Call J, Watzek J, Brosnan S, Aureli F (2018) Social inhibition and behavioural flexibility when the context changes: a comparison across six primate species. Sci Rep 8:3067 . https://doi.org/10.1038/s41598-018-21496-6

Anderson B (1993) Evidence from the rat for a general factor that underlies cognitive performance and that relates to brain size: intelligence? Neurosci Lett 153:98-102 . https://doi.org/10.1016/0304-3940(93)90086-Z

Anderson RC, Searcy WA, Peters S, Hughes M, DuBois AL, Nowicki S (2017) Song learning and cognitive ability are not consistently related in a songbird. Anim Cogn 20:309-320 . https://doi.org/10.1007/s10071-016-1053-7

Andriatsitohaina B, Ramsay MS, Kiene F, Lehman SM, Rasoloharijaona S, Rakotondravony R, Radespiel U (2020) Ecological fragmentation effects in mouse lemurs and small mammals in northwestern Madagascar. Am J Primatol 82:1-11 . https://doi.org/10.1002/ajp.23059

Aplin LM (2019) Culture and cultural evolution in birds: a review of the evidence. Anim Behav 147:179-187 . https://doi.org/10.1016/j.anbehav.2018.05.001

Arden R, Adams MJ (2016) A general intelligence factor in dogs. Intelligence 55:79-85 . https://doi.org/10.1016/j.intell.2016.01.008

Ashton BJ, Ridley AR, Edwards EK, Thornton A (2018a) Cognitive performance is linked to group size and affects fitness in Australian magpies. Nature 554:364-367. https://doi.org/10.1038/nature25503

Ashton BJ, Thornton A, Ridley AR (2018b) An intraspecific appraisal of the social intelligence hypothesis. Phil Trans R Soc B 373:20170288 . https://doi.org/10.1098/rstb.2017.0288

Ashton BJ, Thornton A, Ridley AR (2019) Larger group sizes facilitate the emergence and spread of innovations in a group-living bird. Anim Behav 158:1-7 . https://doi.org/10.1016/j.anbehav.2019.10.004

Atsalis S (1999) Seasonal fluctuations in body fat and activity levels in a rain-forest species of mouse Lemur, Microcebus rufus. Int J Primatol 20:883-910 . https://doi.org/10.1023/A:1020826502103

Audet J, Kayello L, Ducatez S, Perillo S, Cauchard L, Howard JT, O'Connell LA, Jarvis ED, Lefebvre L (2018) Divergence in problem-solving skills is associated with differential expression of glutamate receptors in wild finches. Sci Adv 4:eaao6369. https://doi.org/10.1126/sciadv.aao6369

Aureli F, Schaffner CM, Boesch C, Bearder SK, Call J, Chapman CA, Connor R, Fiore A Di, Dunbar RIM, Henzi SP, Holekamp K, Korstjens AH, Layton R, Lee P, Lehmann J, Manson JH, RamosFernandez G, Strier KB, Schaik CP van (2008) Fission-fusion dynamics. Curr Anthropol 49:627-654 . https://doi.org/10.1086/586708

Banerjee K, Chabris CF, Johnson VE, Lee JJ, Tsao F, Hauser MD (2009) General intelligence in another primate: Individual differences across cognitive task performance in a New World monkey (Saguinus oedipus). PLoS ONE 4:e5883 . https://doi.org/10.1371/journal.pone.0005883

Barkley CL, Jacobs LF (2007) Sex and species differences in spatial memory in food-storing 


\section{References}

kangaroo rats. Anim Behav 73:321-329 . https://doi.org/10.1016/j.anbehav.2006.07.009

Barrett L (2014) What counts as (non) cognitive? A comment on Rowe and Healy. Behav Ecol 25:1293-1294 . https://doi.org/10.1093/beheco/aru114

Barton RA (1996) Neocortex size and behavioural ecology in primates. Proc R Soc B 263:173-177 . https://doi.org/10.1098/rspb.1996.0028

Barton RA, Harvey PH (2000) Mosaic evolution of brain structure in mammals. Nature 405:10551058. https://doi.org/10.1038/35016580

Barton RA, Purvis A, Harvey PH (1995) Evolutionary radiation of visual and olfactory brain system in primates, bats and insectivores. Phil Trans $R$ Soc B 348:381-392. https://doi.org/10.1098/rstb.1995.0076

Bayne T, Brainard D, Byrne RW, Chittka L, Clayton NS, Heyes C, Mather J, Ölveczky B, Shadlen M, Suddendorf T, Webb B (2019) What is cognition? Curr Biol 29:R608-R615 . https://doi.org/10.1016/j.cub.2019.05.044

Beauchamp G, Fernández-Juricic E (2004) Is there a relationship between forebrain size and group size in birds? Evol Ecol Res 6:833-842

Bednekoff PA, Balda RP (1996) Observational spatial memory in Clark's nutcrackers and Mexican jays. Anim Behav 52:833-839 . https://doi.org/10.1006/anbe.1996.0228

Bednekoff PA, Balda RP, Kamil AC, Hile AG (1997) Long-term spatial memory in four seed-caching corvid species. Anim Behav 53:335-341 . https://doi.org/10.1006/anbe.1996.0395

Benson-Amram S, Dantzer B, Stricker G, Swanson EM, Holekamp KE (2016) Brain size predicts problem-solving ability in mammalian carnivores. P Natl Acad Sci USA 113:2532-2537 . https://doi.org/10.1073/pnas.1505913113

Benson-Amram S, Holekamp KE (2012) Innovative problem solving by wild spotted hyenas. Proc R Soc B 279:4087-4095 . https://doi.org/10.1098/rspb.2012.1450

Beran MJ (2015) The comparative science of "self-control" what are we talking about? Front Psychol 6:939-944 . https://doi.org/10.3389/fpsyg.2015.00051

Bieberich C (2007) Microtus oeconomus. In: Anim Divers Web. https://animaldiversity.org/accounts/Microtus_oeconomus/. Accessed 12 Sep 2020

BirdLife International (2017) Poecile palustris. IUCN Red List Threat Species 2017 eT22735995A118837418 8235

BirdLife International (2019) Poecile montanus. IUCN Red List Threat Species 2019 eT155139697A155139155 8235: . https://doi.org/10.2305/IUCN.UK.20193.RLTS.T155139697A155139155.en

Bókony V, Lendvai ÁZ, Vágási CI, Pătraş L, Pap PL, Németh J, Vincze E, Papp S, Preiszner B, Seress G, Liker A, Pa L, Sheffield S (2014) Necessity or capacity? Physiological state predicts problem-solving performance in house sparrows. Behav Ecol 25:124-135 . https://doi.org/10.1093/beheco/art094

Bolnick DI, Svanbäck R, Fordyce JA, Yang LH, Davis JM, Hulsey CD, Forister ML (2003) The ecology of individuals: Incidence and implications of individual specialization. Am Nat 161:1-28 . https://doi.org/10.1086/343878

Bolnick DI, Yang LH, Fordyce JA, Davis JM, Svanbäck R (2002) Measuring individual-level resource specialization. Ecology 83:2936-2941 . $\quad$ https://doi.org/10.1890/00129658(2002)083[2936:MILRS]2.0.CO;2

Bond AB, Kamil AC, Balda RP (2003) Social complexity and transitive inference in corvids. Anim Behav 65:479-487 . https://doi.org/10.1006/anbe.2003.2101

Bond AB, Kamil AC, Balda RP (2007) Serial reversal learning and the evolution of behavioral flexibility in three species of North American corvids (Gymnorhinus cyanocephalus, Nucifraga columbiana, Aphelocoma californica). J Comp Psychol 121:372-379 . https://doi.org/10.1037/07357036.121.4.372

Bons N, Rieger F, Prudhomme D, Fisher A, Krause KH (2006) Microcebus murinus: A useful primate model for human cerebral aging and Alzheimer's disease? Genes, Brain Behav 5:120- 
130 . https://doi.org/10.1111/j.1601-183X.2005.00149.x

Bonté E, Kemp C, Fagot J (2014) Age effects on transfer index performance and executive control in baboons (Papio papio). Front Psychol 5:1-7 . https://doi.org/10.3389/fpsyg.2014.00188

Boogert NJ, Anderson RC, Peters S, Searcy WA, Nowicki S (2011) Song repertoire size in male song sparrows correlates with detour reaching, but not with other cognitive measures. Anim Behav 81:1209-1216 . https://doi.org/10.1016/j.anbehav.2011.03.004

Boubli J, Alves SL, Buss G, Calouro AM, Carvalho A, N. C-M, Heymann EW, Lynch Alfaro J, Martins AB, Messias M, Mittermeier RA, Mollinedo J, Moscoso P, Palacios E, Ravetta, A., Rumiz DI, Rylands AB, Shanee S, Stevenson PR, de la Torre S, Urbani B (2020) Sapajus apella. IUCN Red List Threat Species 2020 eT172351505A172353050. https://doi.org/10.2305/IUCN.UK.2020-2.RLTS.T172351505A172353050.en 10

Boyd R, Richerson PJ (1996) Why culture is common, but cultural evolution is rare. Proc Br Acad 88:77-93

Bräuer J, Hanus D, Pika S, Gray R, Uomini N (2020) Old and new approaches to animal cognition: There is not "one cognition." J Intell 8:28 . https://doi.org/10.3390/jintelligence8030028

Brian A, Venables B, Bates DM, Hornik K, Gebhardt A, Firth D (2021) Support functions and datasets for venables and ripley's MASS. CRAN Repos

Brown C, Braithwaite VA (2005) Effects of predation pressure on the cognitive ability of the poeciliid Brachyraphis episcopi. Behav Ecol 16:482-487 . https://doi.org/10.1093/beheco/ari016

Buechel SD, Boussard A, Kotrschal A, van der Bijl W, Kolm N (2018) Brain size affects performance in a reversal-learning test. Proc R Soc B 285:20172031 . https://doi.org/10.1098/rspb.2017.2031

Burkart JM, Schubiger MN, van Schaik CP (2017) The evolution of general intelligence. Behav Brain Sci 40:e195 . https://doi.org/10.1017/S0140525X16000959

Burns JG, Rodd FH (2008) Hastiness, brain size and predation regime affect the performance of wild guppies in a spatial memory task. Anim Behav 76:911-922 . https://doi.org/10.1016/j.anbehav.2008.02.017

Byrne RW, Bates LA (2010) Primate social cognition: uniquely primate, uniquely social, or just unique? Neuron 65:815-830 . https://doi.org/10.1016/j.neuron.2010.03.010

Byrne RW, Bates LA (2006) Why are animals cognitive? Curr Biol 16:445-448 . https://doi.org/10.1016/j.cub.2006.05.040

Cassola F (2016) Dipodomys microps. In: IUCN Red List Threat Species 2016eT42603A22227645

Cauchard L, Angers B, Boogert NJ, Lenarth M, Bize P, Doligez B (2017) An experimental test of a causal link between problem-solving performance and reproductive success in wild great tits. Front Ecol Evol 5:1-8 . https://doi.org/10.3389/fevo.2017.00107

Cauchoix M, Chaine AS, Barragan-Jason G (2020) Cognition in context: Plasticity in cognitive performance in response to ongoing environmental variables. Front Ecol Evol 8:1-8 . https://doi.org/10.3389/fevo.2020.00106

Cauchoix M, Chow PKY, Van Horik JO, Atance CM, Barbeau EJ, Barragan-Jason G, Bize P, Boussard A, Buechel SD, Cabirol A, Cauchard L, Claidière N, Dalesman S, Devaud JM, Didic M, Doligez B, Fagot J, Fichtel C, Henke-von der Malsburg J, Hermer E, Huber L, Huebner F, Kappeler PM, Klein S, Langbein J, Langley EJG, Lea SEG, Lihoreau M, Lovlie H, Matzel LD, Nakagawa S, Nawroth C, Oesterwind S, Sauce B, Smith EA, Sorato E, Tebbich S, Wallis LJ, Whiteside MA, Wilkinson A, Chaine AS, Morand-Ferron J (2018) The repeatability of cognitive performance: A meta-analysis. Phil Trans R Soc B 373: https://doi.org/10.1098/rstb.2017.0281

Cauchoix M, Hermer E, Chaine AS, Morand-Ferron J (2017) Cognition in the field: Comparison of reversal learning performance in captive and wild passerines. Sci Rep 7:1-10 . https://doi.org/10.1038/s41598-017-13179-5

Chittka L, Niven J (2009) Are Bigger Brains Better? Curr Biol 19:995-1008 . https://doi.org/10.1016/j.cub.2009.08.023

Chow PKY, Clayton NS, Steele MA (2021) Cognitive performance of wild Eastern gray squirrels 


\section{References}

(Sciurus carolinensis) in rural and urban, native, and non-native environments. Front Ecol Evol 9:1-15 . https://doi.org/10.3389/fevo.2021.615899

Chow PKY, Lea SEG, Leaver LA (2016) How practice makes perfect: The role of persistence, flexibility and learning in problem-solving efficiency. Anim Behav 112:273-283 . https://doi.org/10.1016/j.anbehav.2015.11.014

Clarin TMA, Ruczyński I, Page RA, Siemers BM (2013) Foraging ecology predicts learning performance in insectivorous bats. PLoS ONE 8:e64823. https://doi.org/10.1371/journal.pone.0064823

Clary D, Kelly DM (2011) Cache protection strategies of a non-social food-caching corvid, Clark's nutcracker (Nucifraga columbiana). Anim Cogn 14:735-744 . https://doi.org/10.1007/s10071-0110408-3

Clayton NS (1998) Memory and the hippocampus in food-storing birds: A comparative approach. Neuropharmacology 37:441-452 . https://doi.org/10.1016/S0028-3908(98)00037-9

Clayton NS, Krebs JR (1994a) Memory for spatial and object-specific cues in food-storing and nonstoring birds. J Comp Physiol A 174:371-379 . https://doi.org/10.1007/BF00240218

Clayton NS, Krebs JR (1994b) One-trial associative memory: comparison of food-storing and nonstoring species of birds. Anim Learn Behav 22:366-372 . https://doi.org/10.3758/BF03209155

Clutton-Brock TH, Harvey PH (1980) Primates, brains and ecology. J Zool 190:309-323 . https://doi.org/10.1111/j.1469-7998.1980.tb01430.x

Cole EF, Morand-Ferron J, Hinks AE, Quinn JL (2012) Cognitive ability influences reproductive life history variation in the wild. Curr Biol 22:1808-1812 . https://doi.org/10.1016/j.cub.2012.07.051

Cole FR, Wilson DE (2006) Leptonycteris yerbabuenae. Mamm Species 797:1-7 . https://doi.org/10.1644/797.1

Colwell RK, Futuyma DJ (1971) On the measurement of niche breadth and overlap. Ecology 52:567576 . https://doi.org/10.2307/1934144

Cosmides L, Tooby J (1994) Origins of domain specificity: The evolution of functional organization. In: Hirschfeld L, Gelman S (eds) Mapping the Mind: Domin specificity in cognition and culture. Cambridge University Press, Cambridge, pp 85-116

Cristol DA, Reynolds EB, Leclerc JE, Donner AH, Farabaugh CS, Ziegenfus CWS (2003) Migratory dark-eyed juncos, Junco hyemalis, have better spatial memory and denser hippocampal neurons than nonmigratory conspecifics. Anim Behav 66:317-328 . https://doi.org/10.1006/anbe.2003.2194

Croston R, Branch CL, Pitera AM, Kozlovsky DY, Bridge ES, Parchman TL, Pravosudov V V. (2017) Predictably harsh environment is associated with reduced cognitive flexibility in wild foodcaching mountain chickadees. Anim Behav 123:139-149 . https://doi.org/10.1016/j.anbehav.2016.10.004

Daly M, Rauschenberger J, Behrends P (1982) Food aversion learning in kangaroo rats: A specialistgeneralist comparison. Anim Learn Behav 10:314-320 . https://doi.org/10.3758/BF03213716

Damerius LA, Burkart JM, van Noordwijk MA, Haun DBM, Kosonen ZK, Galdikas BMF, Saraswati Y, Kurniawan D, van Schaik CP (2019) General cognitive abilities in orangutans (Pongo abelii and Pongo pygmaeus). Intelligence 74:3-11 . https://doi.org/10.1016/j.intell.2018.10.008

Dammhahn M (2012) Are personality differences in a small iteroparous mammal maintained by a life-history trade-off? Proc Biol Sci 279:2645-51 . https://doi.org/10.1098/rspb.2012.0212

Dammhahn M, Almeling L (2012) Is risk taking during foraging a personality trait? A field test for cross-context consistency in boldness. Anim Behav 84:131-1139. https://doi.org/10.1016/j.anbehav.2012.08.014

Dammhahn M, Kappeler PM (2008a) Small-scale coexistence of two mouse lemur species (Microcebus berthae and M. murinus) within a homogeneous competitive environment. Oecologia 157:473-483 . https://doi.org/10.1007/s00442-008-1079-x

Dammhahn M, Kappeler PM (2008b) Comparative feeding ecology of sympatric Microcebus berthae 
and M. murinus. Int J Primatol 29:1567-1589 . https://doi.org/10.1007/s10764-008-9312-3

Dammhahn M, Kappeler PM (2005) Social system of Microcebus berthae, the world's smallest primate. Int J Primatol 26:407-435 . https://doi.org/10.1007/s10764-005-2931-z

Dammhahn M, Kappeler PM (2012) Seasonality and behavioral energy strategies in Microcebus berthae and M. murinus. In: Masters JC, Gamba M, Génin F (eds) Leaping Ahead. Springer New York, New York, NY, pp 215-223

Davis SJ, Schapiro SJ, Lambeth SP, Wood LA, Whiten A (2019) Behavioral conservatism is linked to complexity of behavior in chimpanzees (Pan troglodytes): Implications for cognition and cumulative culture. J Comp Psychol 133:20-35 . https://doi.org/10.1037/com0000123

Day LB, Crews D, Wilczynski W (1999a) Spatial and reversal learning in congeneric lizards with different foraging strategies. Anim Behav 57:393-407 . https://doi.org/10.1006/anbe.1998.1007

Day LB, Crews D, Wilczynski W (1999b) Relative medial and dorsal cortex volume in relation to foraging ecology in congeneric lizards. Brain Behav Evol 54:314-322 . https://doi.org/10.1159/000006631

Day RL, Coe RL, Kendal JR, Laland KN (2003) Neophilia, innovation and social learning: a study of intergeneric differences in callitrichid monkeys. Anim Behav 65:559-571 . https://doi.org/10.1006/anbe.2003.2074

De Waal FBM (1999) Cultural primatology comes of age. Nature 399:635-636 . https://doi.org/10.1038/21310

Deaner RO, Barton RA, van Schaik CP (2003) Primate brains and life histories: Renewing the connection. In: Kappeler PM, Pereira ME (eds) Primates life histories and socioecology. The University of Chicago Press, Chicago, pp 233-265

Deaner RO, Isler K, Burkart JM, van Schaik CP (2007) Overall brain size, and not encephalization quotient, best predicts cognitive ability across non-human primates. Brain Behav Evol 70:115124 . https://doi.org/10.1159/000102973

Deaner RO, Nunn CL, van Schaik CP (2000) Comparative tests of primate cognition: Different scaling methods produce different results. Brain Behav Evol 55:44-52 . https://doi.org/10.1159/000006641

Deaner RO, van Schaik CP, Johnson V, Robert O, van Schaik CP, Johnson V (2006) Do some taxa have better domain-general cognition than others? A meta-analysis of nonhuman primate studies. Evol Psychol 4:147470490600400 . https://doi.org/10.1177/147470490600400114

DeCasien AR, Higham JP (2019) Primate mosaic brain evolution reflects selection on sensory and cognitive specialization. Nat Ecol Evol 3:1483-1493 . https://doi.org/10.1038/s41559-019-09690

DeCasien AR, Williams SA, Higham JP (2017) Primate brain size is predicted by diet but not sociality. Nat Ecol Evol 1:112 . https://doi.org/10.1038/s41559-017-0112

Devictor V, Clavel J, Julliard R, Lavergne S, Mouillot D, Thuiller W, Venail P, Villéger S, Mouquet N (2010) Defining and measuring ecological specialization. J Appl Ecol 47:15-25 . https://doi.org/10.1111/j.1365-2664.2009.01744.x

Dewar RE, Richard AF (2007) Evolution in the hypervariable environment of Madagascar. P Natl Acad Sci USA 104:13723-13727 . https://doi.org/10.1073/pnas.0704346104

Dicke U, Roth G (2016) Neuronal factors determining high intelligence. Phil Trans R Soc B 371:20150180 . https://doi.org/10.1098/rstb.2015.0180

Dietz C, Kiefer A (2016) Bats of Britain and Europe. Bloomsbury Publishing, London

Dietz JM, Peres CA, Pinder L (1997) Foraging ecology and use of space in wild golden lion tamarins (Leontopithecus rosalia). Am J Primatol 41:289-305 . https://doi.org/10.1002/(SICI)10982345(1997)41:4<289::AID-AJP2>3.0.CO;2-T

Dingle H (2014) Migration. Oxford University Press, Oxford

Dinnen S, Ware S (2020) Conservation research, policy and practice. Cambridge University Press

Domjan M, Galef BG (1983) Biological constraints on instrumental and classical conditioning: Retrospect and prospect. Anim Learn Behav 11:151-161 . https://doi.org/10.3758/BF03199643 


\section{References}

Dougherty LR, Guillette LM, Guillette LM (2018) Linking personality and cognition: a metaanalysis. Phil Trans R Soc B 373:20170282 . https://doi.org/10.1098/rstb.2017.0282

Du Toit N, Pillay N, Ganem G, Rekton C (2016) A conservation assessment of Rhabdomys spp. Red List Mamm South Africa 1-6

Ducatez S, Audet JN, Lefebvre L (2014a) Problem-solving and learning in Carib grackles: individuals show a consistent speed-accuracy trade-off. Anim Cogn 18:485-496 . https://doi.org/10.1007/s10071-014-0817-1

Ducatez S, Clavel J, Lefebvre L (2015) Ecological generalism and behavioural innovation in birds: technical intelligence or the simple incorporation of new foods? J Anim Ecol 84:79-89 . https://doi.org/10.1111/1365-2656.12255

Ducatez S, Lefebvre L, Sayol F, Audet J-NN, Sol D (2020a) Host cognition and parasitism in birds: A review of the main mechanisms. Front Ecol Evol 8:1-102 . https://doi.org/10.3389/fevo.2020.00102

Ducatez S, Sol D, Sayol F, Lefebvre L (2020b) Behavioural plasticity is associated with reduced extinction risk in birds. Nat Ecol Evol 4:788-793 . https://doi.org/10.1038/s41559-020-1168-8

Ducatez S, Tingley R, Shine R (2014b) Using species co-occurrence patterns to quantify relative habitat breadth in terrestrial vertebrates. Ecosphere 5:1-12 . https://doi.org/10.1890/ES1400332.1

Dukas R (2004) Evolutionary Biology of Animal Cognition. Annu Rev Ecol Evol Syst 35:347-374 . https://doi.org/10.1146/annurev.ecolsys.35.112202.130152

Dukas R (1998) Evolutionary ecology of learning. In: Cognitive Ecology: The Evolutionary Ecology of Information Processing and Decision Making. pp 129-174

Dukas R, Ratcliffe JM (2009) Cognitive ecology II. The University of Chicago Press, Chicago

Dunbar RIM (1998) The social brain hypothesis. Evol Anthropol 6:178-190 . https://doi.org/10.1002/(SICI)1520-6505(1998)6:5<178::AID-EVAN5>3.0.CO;2-8

Dunbar RIM, Bever J (2010) Neocortex size predicts group size in carnivores and some insectivores. Ethology 104:695-708 . https://doi.org/10.1111/j.1439-0310.1998.tb00103.x

Dunbar RIM, Shultz S (2007) Understanding primate brain evolution. Phil Trans R Soc B 362:649658 . https://doi.org/10.1098/rstb.2006.2001

Dunbar RIM, Shultz S (2017) Why are there so many explanations for primate brain evolution? Phil Trans R Soc B 372:20160244 . https://doi.org/10.1098/rstb.2016.0244

Dunbar RIM, Shultz S, Press H, York N, Nw A (2007) Evolution in the social brain. Science 317:13441347 . https://doi.org/10.1126/science.1145463

Dunn JC, Cole EF, Quinn JL (2011) Personality and parasites : sex-dependent associations between avian malaria infection and multiple behavioural traits. Behav Ecol Sociobiol 65:1459-1471 . https://doi.org/10.1007/s00265-011-1156-8

Eberle M, Kappeler PM (2002) Mouse lemurs in space and time: A test of the socioecological model. Behav Ecol Sociobiol 51:131-139 . https://doi.org/10.1007/s002650100409

Eberle M, Kappeler PM (2006) Family insurance: Kin selection and cooperative breeding in a solitary primate (Microcebus murinus). Behav Ecol Sociobiol 60:582-588. https://doi.org/10.1007/s00265-006-0203-3

Eberle M, Kappeler PM (2004) Sex in the dark: Determinants and consequences of mixed male mating tactics in Microcebus murinus, a small solitary nocturnal primate. Behav Ecol Sociobiol 57:77-90 . https://doi.org/10.1007/s00265-004-0826-1

Emery NJ, Clayton NS (2004) The mentality of crows: convergent evolution of intelligence in corvids and apes. Science 306:1903-1907 . https://doi.org/10.1126/science.1098410

Estrada A, Garber PA, Rylands AB, Roos C, Fernandez-duque E, Fiore A Di, Nekaris KA, Nijman V, Heymann EW, Lambert JE, Rovero F, Barelli C, Setchell JM, Gillespie TR, Mittermeier RA, Arregoitia LV (2017) Impending extinction crisis of the world's primates: Why primates matter. https://doi.org/10.1126/sciadv.1600946

Eudey A, Kumar A, Singh M, Boonratana R (2020) Macaca fascicularis. IUCN Red List Threat Species 
2.RLTS.T12551A17949449.en

Fagot J, Bonté E (2010) Automated testing of cognitive performance in monkeys: Use of a battery of computerized test systems by a troop of semi-free-ranging baboons (Papio papio). Behav Res Methods 42:507-516 . https://doi.org/10.3758/BRM.42.2.507

Farashahi S, Xu J, Wu S-WW, Soltani A (2020) Learning arbitrary stimulus-reward associations for naturalistic stimuli involves transition from learning about features to learning about objects. Cognition 1-34 . https://doi.org/10.1016/j.cognition.2020.104425

Fernandes HBFF, Woodley of Menie MA, te Nijenhuis J (2014) Differences in cognitive abilities among primates are concentrated on G: Phenotypic and phylogenetic comparisons with two meta-analytical databases. Intelligence 46:311-322 . https://doi.org/10.1016/j.intell.2014.07.007

Ferrari MCO (2014) Short-term environmental variation in predation risk leads to differential performance in predation-related cognitive function. Anim Behav 95:9-14 . https://doi.org/10.1016/j.anbehav.2014.06.001

Fichtel C, Dinter K, Kappeler PM (2020) The lemur baseline: how lemurs compare to monkeys and apes in the Primate Cognition Test Battery. PeerJ 8:e10025 . https://doi.org/10.7717/peerj.10025

Finarelli JA, Flynn JJ (2009) Brain-size evolution and sociality in Carnivora. P Natl Acad Sci USA 106:9345-9349 . https://doi.org/10.1073/pnas.0901780106

Forstmeier W, Schielzeth H (2011) Cryptic multiple hypotheses testing in linear models: Overestimated effect sizes and the winner's curse. Behav Ecol Sociobiol 65:47-55 . https://doi.org/10.1007/s00265-010-1038-5

Fox J, Weisberg S (2011) An R companion to applied regression, 2nd edn.

Franks VR, Thorogood R (2018) Older and wiser? Age differences in foraging and learning by an endangered passerine. Behav Process 148:1-9 . https://doi.org/10.1016/j.beproc.2017.12.009

Friard O, Gamba M (2016) BORIS: a free, versatile open-source event-logging software for video/audio coding and live observations. Methods Ecol Evol 7:1325-1330. https://doi.org/10.1111/2041-210X.12584

Futuyma DJ, Moreno G (1988) The evolution of ecological specialization. Annu Rev Ecol Syst Vol 19 19:207-233 . https://doi.org/10.1146/annurev.es.19.110188.001231

Galef BG, National G The question of animal culture. 3:157-178

Galsworthy MJ, Arden R, Chabris CF (2014) Animal models of general cognitive ability for genetic research into cognitive functioning. In: Finkel D, Reynolds C (eds) Behavior Genetics of Cognition Across the Lifespan. Springer New York, New York, NY, pp 257-278

Galsworthy MJ, Paya-Cano JL, Monleón S, Plomin R (2002) Evidence for general cognitive ability (g) in heterogeneous stock mice and an analysis of potential confounds. Genes, Brain Behav 1:88-95 . https://doi.org/10.1034/j.1601-183X.2002.10204.x

Ganem G, Dufour CMS, Avenant NL, Caminade P, Eiseb SJ, Tougard C, Pillay N (2020) An update on the distribution and diversification of Rhabdomys sp. (Muridae, Rodentia). J Vertebr Biol 69:1 . https://doi.org/10.25225/jvb.20013

Ganem G, Meynard CN, Perigault M, Lancaster J, Edwards S, Caminade P, Watson J, Pillay N (2012) Environmental correlates and co-occurrence of three mitochondrial lineages of striped mice (Rhabdomys) in the Free State Province (South Africa). Acta Oecologica 42:30-40 . https://doi.org/10.1016/j.actao.2012.01.003

Ganzhorn JU, Hapke A, Lahann P, Raharivololona B, Ramanamanjato J-B, Refaly E, Schmid J, Schad J, Sommer S (2012) Population genetics, parasitism, and long-term population dynamics of Microcebus murinus in littoral forest fragments of South-Eastern Madagascar. In: Leaping Ahead. Springer New York, New York, NY, pp 61-69

Ganzhorn JU, Schmid J (1998) Different population dynamics of Microcebus murinus in primary and secondary deciduous dry forests of Madagascar. Int J Primatol 19:785-796 . https://doi.org/10.1023/A:1020337211827

Garamszegi LZ, Erritzøe J, Møller AP (2007) Feeding innovations and parasitism in birds. Biol J 


\section{References}

Linn Soc 90:441-455 . https://doi.org/10.1111/j.1095-8312.2007.00733.x

Gibson BM, Kamil AC (2005) The fine-grained spatial abilities of three seed-caching corvids. Learn Behav 33:59-66 . https://doi.org/10.3758/bf03196050

Gigerenzer G (2008) Why heuristics work. Perspect Psychol Sci 3:20-29 . https://doi.org/10.1111/j.1745-6916.2008.00058.x

Gingins S, Bshary R (2016) The cleaner wrasse outperforms other labrids in ecologically relevant contexts, but not in spatial discrimination. Anim Behav 115:145-155 . https://doi.org/10.1016/j.anbehav.2016.02.022

Gottfredson LS (1997) Why g matters: The complexity of everyday life. Intelligence 24:79-132 . https://doi.org/10.1016/S0160-2896(97)90014-3

Greenberg R (2003) The role of neophobia and neophilia in the development of innovative behavior of birds. In: Reader SM, Laland KN (eds) Animal Innovation. Oxford University Press, pp 175-196

Greenberg R (1983) The role of neophobia in determining the degree of foraging specialization in some migrant warblers. Am Nat 122:444-453

Greggor AL, Clayton NS, Phalan B, Thornton A (2014) Comparative cognition for conservationists. Trends Ecol Evol 29:1-7 . https://doi.org/10.1016/j.tree.2014.06.004

Griffin AS (2016) Innovativeness as an emergent property: a new alignment of comparative and experimental research on animal innovation. Phil Trans R Soc B 371:20150544 . https://doi.org/10.1098/rstb.2015.0544

Griffin AS, Diquelou MC (2015) Innovative problem solving in birds: a cross-species comparison of two highly successful passerines. Anim Behav 100:84-94. https://doi.org/10.1016/j.anbehav.2014.11.012

Griffin AS, Guez D (2014) Innovation and problem solving: A review of common mechanisms. Behav Process 109:121-134 . https://doi.org/10.1016/j.beproc.2014.08.027

Griffin AS, Guillette LM, Healy SD, Griffin AS, Guillette LM, Healy SD (2015) Cognition and personality: An analysis of an emerging field. Trends Ecol Evol 30:1-8 . https://doi.org/10.1016/j.tree.2015.01.012

Guenther A, Brust V, Dersen M, Trillmich F (2014) Learning and personality types are related in cavies (Cavia aperea). J Comp Psychol 128:74-81 . https://doi.org/10.1037/a0033678

Guez D, Griffin AS (2016) Unraveling the key to innovative problem solving: A test of learning versus persistence. Behav Ecol 27:1449-1460 . https://doi.org/10.1093/beheco/arw055

Guillette LM, Hahn AH, Hoeschele M, Przyslupski AM, Sturdy CB (2015) Individual differences in learning speed, performance accuracy and exploratory behaviour in black-capped chickadees. Anim Cogn 18:165-178 . https://doi.org/10.1007/s10071-014-0787-3

Hafner MS, Hafner JC (1984) Brain size, adaptation and heterochrony in geomyoid rodents. Evolution 38:1088-1098 . https://doi.org/10.1111/j.1558-5646.1984.tb00378.x

Halsey LG, Bezerra BM, Souto AS (2006) Can wild common marmosets (Callithrix jacchus) solve the parallel strings task? Anim Cogn 9:229-233 . https://doi.org/10.1007/s10071-006-0016-9

Hämäläinen A, Dammhahn M, Aujard F, Eberle M, Hardy I, Kappeler PM, Perret M, SchlieheDiecks S, Kraus C (2014a) Senescence or selective disappearance? Age trajectories of body mass in wild and captive populations of a small-bodied primate. Proc R Soc B 281:20140830 . https://doi.org/10.1098/rspb.2014.0830

Hämäläinen A, Heistermann M, Fenosoa ZSE, Kraus C (2014b) Evaluating capture stress in wild gray mouse lemurs via repeated fecal sampling: Method validation and the influence of prior experience and handling protocols on stress responses. Gen Comp Endocrinol 195:68-79 . https://doi.org/10.1016/j.ygcen.2013.10.017

Harrell FE (2021) Harrell Miscellaneous. CRAN Repos

Hartwig W, Rosenberger AL, Norconk MA, Owl MY (2011) Relative brain size, gut size, and evolution in New World Monkeys. Anat Rec 294:2207-2221 . https://doi.org/10.1002/ar.21515

Harvey PH, Clutton-Brock TH, Mace GM (1980) Brain size and ecology in small mammals and 
primates. J Zool 77:4387-4389

Haupt M, Eccard JA, Winter Y (2010) Does spatial learning ability of common voles (Microtus arvalis) and bank voles (Myodes glareolus) constrain foraging efficiency? Anim Cogn 13:783791 . https://doi.org/10.1007/s10071-010-0327-8

Hauser MD (1999) Perseveration, inhibition and the prefrontal cortex: A new look. Curr Opin Neurobiol 9:214-222 . https://doi.org/10.1016/S0959-4388(99)80030-0

Healy SD, Bacon IEE, Haggis O, Harris APP, Kelley LAA (2009) Explanations for variation in cognitive ability: Behavioural ecology meets comparative cognition. Behav Process 80:288294 . https://doi.org/10.1016/j.beproc.2008.10.002

Healy SD, Rowe C (2007) A critique of comparative studies of brain size. Proc R Soc B 274:453-464 . https://doi.org/10.1098/rspb.2006.3748

Healy SD, Suhonen J (1996) Memory for locations of stored food in willow tits and marsh tits. Behaviour 133:71-80 . https://doi.org/10.1163/156853996X00035

Heldstab SA, Kosonen ZK, Koski SE, Burkart JM, van Schaik CP, Isler K (2016) Manipulation complexity in primates coevolved with brain size and terrestriality. Sci Rep 6:24528 . https://doi.org/10.1038/srep24528

Henke-von der Malsburg J, Fichtel C (2018) Are generalists more innovative than specialists? A comparison of innovative abilities in two wild sympatric mouse lemur species. R Soc Open Sci 5:180480 . https://doi.org/10.1098/rsos.180480

Henke-von der Malsburg J, Kappeler PM, Fichtel C (2020) Linking ecology and cognition: does ecological specialisation predict cognitive test performance? Behav Ecol Sociobiol 74:154 . https://doi.org/10.1007/s00265-020-02923-z

Henrich J, Heine SJ, Norenzayan A (2010) The weirdest people in the world? Behav Brain Sci 33:6183 . https://doi.org/10.1017/S0140525X0999152X

Henry M, Stoner KE (2011) Relationship between spatial working memory performance and diet specialization in two sympatric nectar bats. PLoS ONE 6:e23773 . https://doi.org/10.1371/journal.pone.0023773

Herculano-Houzel S (2017) Numbers of neurons as biological correlates of cognitive capability. Curr Opin Behav Sci 16:1-7 . https://doi.org/10.1016/j.cobeha.2017.02.004

Hernández-Montero JR, Schöner CR, Kerth G (2020) No evidence for memory retention of a learned association between a cue and roost quality after hibernation in free-ranging bats. Ethology 126:761-771 . https://doi.org/10.1111/eth.13029

Herndon JG, Moss MB, Rosene DL, Killiany RJ (1997) Patterns of cognitive decline in aged rhesus monkeys. Behav Brain Res 87:25-34 . https://doi.org/10.1016/S0166-4328(96)02256-5

Herrmann E, Call J (2012) Are there geniuses among the apes? Phil Trans R Soc Lond B 367:27532761 . https://doi.org/10.1098/rstb.2012.0191

Herrmann E, Call J, Hernàndez-Lloreda MV, Hare B, Tomasello M (2007) Humans Have Evolved Specialized Skills of Social Cognition: The Cultural Intelligence Hypothesis. Science 317:13601366 . https://doi.org/10.1126/science.1146282

Hills TT, Todd PM, Lazer D, Redish AD, Couzin ID (2015) Exploration versus exploitation in space, mind, and society. Trends Cogn Sci 19:46-54 . https://doi.org/10.1016/j.tics.2014.10.004

Hoedjes KM, Steidle JLMM, Werren JH, Vet LEMM, Smid HM (2012) High-throughput olfactory conditioning and memory retention test show variation in Nasonia parasitic wasps. Genes, Brain Behav 11:879-887 . https://doi.org/10.1111/j.1601-183X.2012.00823.x

Hoffmeister DF (1957) Review of the long-nosed bats of the genus Leptonycteris. J Mammal 38:454 . https://doi.org/10.2307/1376397

Holekamp KE, Sakai ST, Lundrigan BL (2007) Social intelligence in the spotted hyena (Crocuta crocuta). Phil Trans R Soc B 362:523-538 . https://doi.org/10.1098/rstb.2006.1993

Hopkins WD, Mareno MC, Neal Webb SJ, Schapiro SJ, Raghanti MA, Sherwood CC (2020) Agerelated changes in chimpanzee (Pan troglodytes) cognition: Cross-sectional and longitudinal analyses. Am J Primatol 1-9 . https://doi.org/10.1002/ajp.23214 


\section{References}

Hopkins WD, Russell JL, Schaeffer J (2014) Chimpanzee intelligence is heritable. Curr Biol 24:1649_ 1652 . https://doi.org/10.1016/j.cub.2014.05.076

Howard VWJ (1994) Kangaroo rats. In: Hygnstrom SE, Timm RM, Larson GE (eds) Prevention and Control of Wildlife Damage. University of Nebraska-Lincoln, Lincoln, p B-101-B-104

Huebner F (2020) Cognition in the wild: Individual differences in cognitive abilities and their link with fitness in a wild primate, the grey mouse lemur (Microcebus murinus). Georg-August Universität Göttingen

Huebner F, Fichtel C (2015) Innovation and behavioral flexibility in wild redfronted lemurs (Eulemur rufifrons). Anim Cogn 18:777-787 . https://doi.org/10.1007/s10071-015-0844-6

Huebner F, Fichtel C, Kappeler PM (2018) Linking cognition with fitness in a wild primate: Fitness correlates of problem-solving performance and spatial learning ability. Phil Trans R Soc B 373: . https://doi.org/10.1098/rstb.2017.0295

Hughes JB (2000) The scale of resource specialization and the distribution and abundance of lycaenid butterflies. Oecologia 123:375-383 . https://doi.org/10.1007/s004420051024

Humphrey N (1976) The social function of intellect. In: Bateson PPG, Hinde RA (eds) Growing Points in Ethology. Cambridge University Press, Cambridge, pp 303-317

Humphreys LG (1979) The construct of general intelligence. Intelligence 3:105-120

Hutchinson GE (1957) Concluding remarks. Cold Spring Harb Symp 22:415-427 . https://doi.org/10.1101/SQB.1957.022.01.039

Hutchinson GE (1953) The concept of pattern in ecology. P Acad Nat Sci Phila 105:1-12

Irwin MT, Wright PC, Birkinshaw C, Fisher BL, Gardner CJ, Glos J, Goodman SM, Loiselle P, Rabeson P, Raharison JL, Raherilalao MJ, Rakotondravony D, Raselimanana A, Ratsimbazafy J, Sparks JS, Wilmé L, Ganzhorn JU (2010) Patterns of species change in anthropogenically disturbed forests of Madagascar. Biol Conserv 143:2351-2362. https://doi.org/10.1016/j.biocon.2010.01.023

Isden J, Panayi C, Dingle C, Madden J (2013) Performance in cognitive and problem-solving tasks in male spotted bowerbirds does not correlate with mating success. Anim Behav 86:829-838 . https://doi.org/10.1016/j.anbehav.2013.07.024

Iwaniuk AN, Arnold KE (2004) Is cooperative breeding associated with bigger brains? A comparative test in the Corvida (Passeriformes). Ethology 110:203-220 . https://doi.org/10.1111/j.1439-0310.2003.00957.x

Jackman S, Tahk A, Zeileis A, Maimone C, Fearon J, Meers Z (2020) Political science computational laboratory. CRAN Repos

Jacobs IF, Osvath M (2015) The string-pulling paradigm in comparative psychology. J Comp Psychol 129:89-120 . https://doi.org/10.1037/a0038746

Jensen AR (1985) The nature of the black-white difference on various psychometric tests: Spearman's hypothesis. Behav Brain Sci 8:193-219 . https://doi.org/10.1017/S0140525X00020392

Jerison HJ (1973) Evolution of the brain and intelligence. Academic Press, New York

Jolly A (1966) Lemur social behavior and primate intelligence. Science 153:501-506 . https://doi.org/10.1126/science.153.3735.501

Jolly A (1964) Prosimians' manipulation of simple object problems. Anim Behav 12:560-570 . https://doi.org/10.1016/0003-3472(64)90080-6

Joly M, Ammersdörfer S, Schmidtke D, Zimmermann E (2014) Touchscreen-Based Cognitive Tasks Reveal Age-Related Impairment in a Primate Aging Model, the Grey Mouse Lemur (Microcebus murinus). PLoS ONE 9:e109393 . https://doi.org/10.1371/journal.pone.0109393

Jones S, Paul ES, Dayan P, Robinson ESJJ, Mendl M (2017) Pavlovian influences on learning differ between rats and mice in a counter-balanced Go/NoGo judgement bias task. Behav Brain Res 331:214-224 . https://doi.org/10.1016/j.bbr.2017.05.044

Kabadayi C, Bobrowicz K, Osvath M (2018) The detour paradigm in animal cognition. Anim Cogn 21:21-35 . https://doi.org/10.1007/s10071-017-1152-0 
Kappeler PM (2019) A framework for studying social complexity. Behav Ecol Sociobiol 73:1-14 . https://doi.org/10.1007/s00265-018-2601-8

Keagy J, Savard JF, Borgia G (2011) Complex relationship between multiple measures of cognitive ability and male mating success in satin bowerbirds, Ptilonorhynchus violaceus. Anim Behav 81:1063-1070 . https://doi.org/10.1016/j.anbehav.2011.02.018

Keagy J, Savard JF, Borgia G (2009) Male satin bowerbird problem-solving ability predicts mating success. Anim Behav 78:809-817 . https://doi.org/10.1016/j.anbehav.2009.07.011

Kittler K (2017) ROOTS OF PRIMATE COGNITION The Primate Cognition Test Battery applied to three species of lemurs (Varecia variegata, Lemur catta and Microcebus murinus). Georg-AugustUniversität Göttingen

Kittler K, Kappeler PM, Fichtel C (2018) Instrumental problem-solving abilities in three lemur species (Microcebus murinus, Varecia variegata, and Lemur catta). J Comp Psychol 132:306-314 . https://doi.org/10.1037/com0000113

Kotrschal A, Rogell B, Bundsen A, Svensson B, Zajitschek S, Brännström I, Immler S, Maklakov AA, Kolm N (2013) Artificial selection on relative brain size in the guppy reveals costs and benefits of evolving a larger brain. Curr Biol 23:168-171 . https://doi.org/10.1016/j.cub.2012.11.058

Kraus C, Eberle M, Kappeler PM (2008) The costs of risky male behaviour: sex differences in seasonal survival in a small sexually monomorphic primate. Proc Biol Sci 275:1635-1644 . https://doi.org/10.1098/rspb.2008.0200

Krebs CJ (1989) Niche overlap and diet analysis. In: Pisano S (ed) Ecological methodology. HarperCollinsPublishers, New York, pp 372-377

Krebs JR (1990) Food-storing birds: adaptive specialization in brain and behaviour? Phil Trans R Soc Lond Ser B 329:153-160 . https://doi.org/10.1098/rstb.1990.0160

Kudo H, Dunbar RIMRIM (2001) Neocortex size and social network size in primates. Anim Behav 62:711-722 . https://doi.org/10.1006/anbe.2001.1808

Kumpan LT, Smeltzer EA, Teichroeb JA (2020) Animal cognition in the field: performance of wild vervet monkeys (Chlorocebus pygerythrus) on a reversal learning task. Anim Cogn 23:523-534 . https://doi.org/10.1007/s10071-020-01356-5

Lahann P (2007) Feeding ecology and seed dispersal of sympatric cheirogaleid lemurs (Microcebus murinus, Cheirogaleus medius, Cheirogaleus major) in the littoral rainforest of south-east Madagascar. J Zool 271:88-98 . https://doi.org/10.1111/j.1469-7998.2006.00222.x

Lahann P, Schmid J, Ganzhorn JU (2006) Geographic variation in populations of Microcebus murinus in Madagascar: Resource seasonality or Bergmann's rule? Int J Primatol 27:983-999 . https://doi.org/10.1007/s10764-006-9055-y

Lahti DC (2015) The limits of artificial stimuli in behavioral research: The umwelt gamble. Ethology 121:529-537 . https://doi.org/10.1111/eth.12361

Languille S, Blanc S, Blin O, Canale CI, Dal-Pan A, Devau G, Dhenain M, Dorieux O, Epelbaum J, Gomez D, Hardy I, Henry P-Y, Irving EA, Marchal J, Mestre-Francés N, Perret M, Picq J-L, Pifferi F, Rahman A, Schenker E, Terrien J, Théry M, Verdier J-M, Aujard F (2012) The grey mouse lemur: A non-human primate model for ageing studies. Ageing Res Rev 11:150-162 . https://doi.org/10.1016/j.arr.2011.07.001

Laughlin SB, De Ruyter Van Steveninck RR, Anderson JC (1998) The metabolic cost of neural information. Nat Neurosci 1:36-41 . https://doi.org/10.1038/236

Lea SEG (2020) Behavioral flexibility : A review, a model, and some exploratory tests

Lea SEG, Chow PKY, Leaver LA, McLaren IPL (2020) Behavioral flexibility: A review, a model, and some exploratory tests. Learn Behav 48:173-187 . https://doi.org/10.3758/s13420-020-00421-w

Lee PC (2003) Innovation as a behavioural response to environmental challenges: a cost and benefit approach. In: Reader SM, Laland KN (eds) Animal Innovation. Oxford University Press, Oxford, pp 261-278

Lefebvre L, Reader SM, Sol D (2004) Brains, innovations and evolution in birds and primates. Brain Behav Evol 63:233-246 . https://doi.org/10.1159/000076784 


\section{References}

Lefebvre L, Sol D (2008) Brains, lifestyles and cognition: Are there general trends? Brain Behav Evol 72:135-144 . https://doi.org/10.1159/000151473

Lennie P (2003) The cost of cortical computation. Curr Biol 13:493-497 . https://doi.org/10.1016/S0960-9822(03)00135-0

Liedtke J, Fromhage L (2019) Modelling the evolution of cognitive styles. BMC Evol Biol 19:1-10 . https://doi.org/10.1186/s12862-019-1565-2

Locurto C, Benoit A, Crowley C, Miele A (2006) The structure of individual differences in batteries of rapid acquisition tasks in mice. J Comp Psychol 120:378-388 . https://doi.org/10.1037/07357036.120.4.378

Locurto C, Fortin E, Sullivan R (2003) The structure of individual differences in heterogeneous stock mice across problem types and motivational systems. Genes, Brain Behav 2:40-55 . https://doi.org/10.1034/j.1601-183X.2003.00006.x

Logan CJ (2016) How far will a behaviourally flexible invasive bird go to innovate? R Soc Open Sci 3:160247 . https://doi.org/http://dx.doi.org/10.1098/rsos.160247

Logan CJ, Avin S, Boogert N, Buskell A, Cross FR, Currie A, Jelbert S, Lukas D, Mares R, Navarrete AF, Shigeno S, Montgomery SH (2018) Beyond brain size: Uncovering the neural correlates of behavioral and cognitive specialization. Comp Cogn Behav Rev 13:55-89 . https://doi.org/10.3819/CCBR.2018.130008

Lucas JR, Brodin A, de Kort SR, Clayton NS (2004) Does hippocampal size correlate with the degree of caching specialization? Proc R Soc Lond B 271:2423-2429 . https://doi.org/10.1098/rspb.2004.2912

Lührs ML, Dammhahn M, Kappeler PM, Fichtel C, Lu M, Kappeler PM, Fichtel C (2009) Spatial memory in the grey mouse lemur (Microcebus murinus). Anim Cogn 12:599-609 . https://doi.org/10.1007/s10071-009-0219-y

Lukas D, Clutton-Brock TH (2013) The evolution of social monogamy in mammals. Science 341:526530. https://doi.org/10.1126/science. 1238677

Lundrigan B, Mueller M (2003) Myodes glareolus. In: Anim Divers Web. https://animaldiversity.org/accounts/Myodes_glareolus/. Accessed 12 Sep 2020

MacArthur RH (1957) On the relative abundance of bird species. Zoology 43:293-295

Mace GM, Harvey PH, Clutton-Brock TH (2009) Brain size and ecology in small mammals. J Zool 193:333-354 . https://doi.org/10.1111/j.1469-7998.1981.tb03449.x

Mackay MK, Pillay N (2017) Similarities in spatial cognition in sister species of the striped mouse Rhabdomys originating from different ecological contexts. Behaviour 154:1397-1420 . https://doi.org/10.1163/1568539X-00003474

MacLean EL, Barrickman NL, Johnson EM, Wall CE (2009) Sociality, ecology, and relative brain size in lemurs. J Hum Evol 56:471-478 . https://doi.org/10.1016/j.jhevol.2008.12.005

MacLean EL, Hare B, Nunn CL, Addessi E, Amici F, Anderson RC, Aureli F, Baker JM, Bania AE, Barnard AM, Boogert NJ, Brannon EM, Bray EE, Bray J, Brent LJN, Burkart JM, Call J, Cantlon JF, Cheke LG, Clayton NS, Delgado MM, DiVincenti LJ, Fujita K, Herrmann E, Hiramatsu C, Jacobs LF, Jordan KE, Laude JR, Leimgruber KL, Messer EJE, de A. Moura AC, Ostoji L, Picard A, Platt ML, Plotnik JM, Range F, Reader SM, Reddy RB, Sandel AA, Santos LR, Schumann K, Seed AM, Sewall KB, Shaw RC, Slocombe KE, Su Y, Takimoto A, Tan J, Tao R, van Schaik $\mathrm{CP}$, Viranyi Z, Visalberghi E, Wade JC, Watanabe A, Widness J, Young JK, Zentall TR, Zhao Y (2014) The evolution of self-control. P Natl Acad Sci USA 111:E2140-E2148 . https://doi.org/10.1073/pnas.1323533111

MacLean EL, Matthews LJ, Hare BA, Nunn CL, Anderson RC, Aureli F, Brannon EM, Call J, Drea CM, Emery NJ, Haun DBM, Herrmann E, Jacobs LF, Platt ML, Rosati AG, Sandel AA, Schroepfer KK, Seed AM, Tan J, van Schaik CP, Wobber V (2012) How does cognition evolve? Phylogenetic comparative psychology. Anim Cogn 15:223-238. https://doi.org/10.1007/s10071-011-0448-8

MacLean EL, Merritt DJ, Brannon EM (2008) Social complexity predicts transitive reasoning in 
prosimian primates. Anim Behav 76:479-486 . https://doi.org/10.1016/j.anbehav.2008.01.025

MacLean EL, Sandel AA, Bray J, Oldenkamp RE, Reddy RB, Hare BA (2013) Group size predicts social but not nonsocial cognition in lemurs. PLoS ONE 8:e66359 . https://doi.org/10.1371/journal.pone.0066359

Maille A, Schradin C (2016a) Ecophysiology of cognition: How do environmentally induced changes in physiology affect cognitive performance? Biol Rev 92:1101-1112 . https://doi.org/10.1111/brv.12270

Maille A, Schradin C (2016b) Survival is linked with reaction time and spatial memory in African striped mice. Biol Lett 12: . https://doi.org/10.1098/rsbl.2016.0346

Marine J, Michel B, Deputte B, Verdier JM (2004) Odor discrimination assessment with an automated olfactometric method in a prosimian primate, Microcebus murinus. Physiol Behav 82:325-329 . https://doi.org/10.1016/j.physbeh.2004.03.019

Markolf M, Schäffler L, Kappeler PM (2020) Microcebus berthae. IUCN Red List Threat Species ${ }^{\mathrm{TM}}$ 8235:

Martin AE, Fahrig L (2018) Habitat specialist birds disperse farther and are more migratory than habitat generalist birds. Ecology 99:2058-2066 . https://doi.org/10.1002/ecy.2428

Matzel LD, Han YR, Grossman H, Karnik MS, Patel D, Scott N, Specht SM, Gandhi CC (2003) Individual differences in the expression of a "general" learning ability in mice. J Neurosci 23:6423-33 . https://doi.org/10.1523/JNEUROSCI.23-16-06423.2003

Matzel LD, Wass C, Kolata S (2011) Individual differences in animal intelligence: Learning, reasoning, selective attention and inter-species conservation of a cognitive trait. Int J Comp Psychol 24:

Mazza V, Jacob J, Dammhahn M, Zaccaroni M, Eccard JA (2019) Individual variation in cognitive style reflects foraging and anti-predator strategies in a small mammal. Sci Rep 9:10157 . https://doi.org/10.1038/s41598-019-46582-1

McBride SD, Perentos N, Morton AJ (2016) A mobile, high-throughput semi-automated system for testing cognition in large non-primate animal models of Huntington disease. J Neurosci Methods 265:25-33 . https://doi.org/10.1016/j.jneumeth.2015.08.025

McCabe CM, Reader SM, Nunn CL (2015) Infectious disease, behavioural flexibility and the evolution of culture in primates. Proc R Soc B 282:20140862 . https://doi.org/10.1098/rspb.2014.0862

McCormack JE, Smith TB (2008) Niche expansion leads to small-scale adaptive divergence along an elevation gradient in a medium-sized passerine bird. Proc R Soc B 275:2155-2164 . https://doi.org/10.1098/rspb.2008.0470

Mettke-Hofmann C (2014) Cognitive ecology: ecological factors, life-styles, and cognition. WIRE Cogn Sci 5:345-360 . https://doi.org/10.1002/wcs.1289

Meynard CN, Pillay N, Perrigault M, Caminade P, Ganem G (2012) Evidence of environmental niche differentiation in the striped mouse (Rhabdomys sp.): Inference from its current distribution in southern Africa. Ecol Evol 2:1008-1023 . https://doi.org/10.1002/ece3.219

Miller R, Boeckle M, Jelbert SA, Frohnwieser A, Wascher CAF, Clayton NS (2019) Self-control in crows, parrots and nonhuman primates. WIRE Cogn Sci 10:1-17 . https://doi.org/10.1002/wcs.1504

Milton K (1988) Foraging behaviour and the evolution of primate intelligence. In: Byrne RW, Whiten A (eds) Machiavellian intelligence: Social expertise and the evolution of intellect in monkeys, apes, and humans. Oxford University Press, New York, pp 285-305

Mittermeier RA, Ganzhorn JU, Konstant WR, Glander K, Tattersall I, Groves CP, Rylands AB, Hapke A, Ratsimbazafy J, Mayor MI, Louis EE, Rumpler Y, Schwitzer C, Rasoloarison RM (2008) Lemur diversity in Madagascar. Int J Primatol 29:1607-1656 . https://doi.org/10.1007/s10764-008-9317-y

Mittermeier RA, Louis EE, Richardson M, Schwitzer C, Rylands AB, Hawkins F, Rajaobelina S, Ratsimbazafy J, Rasoloarison RM, Roos C, Kappeler PM, MacKinnon J, Tatterson I, Konstant 


\section{References}

WiR, Meyers DM, Mast RB (2010) Lemurs of Madagascar, 3rd edn.

Møller AP, Erritzøe J, Garamszegi LZ (2005) Covariation between brain size and immunity in birds: Implications for brain size evolution. J Evol Biol 18:223-237 . https://doi.org/10.1111/j.14209101.2004.00805.x

Morand-Ferron J, Cole EF, Quinn JL (2016) Studying the evolutionary ecology of cognition in the wild: A review of practical and conceptual challenges. Biol Rev 91:367-389 . https://doi.org/10.1111/brv.12174

Morand-Ferron J, Hermer E, Jones TB, Thompson MJ (2019) Environmental variability, the value of information, and learning in winter residents. Anim Behav 147:137-145

Morton FB, Altschul D (2019) Data reduction analyses of animal behaviour: avoiding Kaiser's criterion and adopting more robust automated methods. Anim Behav 149:89-95

Navarrete AF, Reader SM, Street SE, Whalen A, Laland KN (2016) The coevolution of innovation and technical intelligence in primates. Phil Trans R Soc Lond B 371:20150186- . https://doi.org/10.1098/rstb.2015.0186

Nicolakakis N, Sol D, Lefebvre L (2003) Behavioural flexibility predicts species richness in birds, but not extinction risk. Anim Behav 65:445-452 . https://doi.org/10.1006/anbe.2003.2085

Noble S (2017) Microtus arvalis. In: Anim Divers Web. https://animaldiversity.org/accounts/Microtus_arvalis/. Accessed 12 Sep 2020

Overington SE, Griffin AS, Sol D, Lefebvre L (2011) Are innovative species ecological generalists? A test in North American birds. Behav Ecol 22:1286-1293. https://doi.org/10.1093/beheco/arr130

Overington SE, Morand-Ferron J, Boogert NJ, Lefebvre L (2009) Technical innovations drive the relationship between innovativeness and residual brain size in birds. Anim Behav 78:10011010 . https://doi.org/10.1016/j.anbehav.2009.06.033

Park CR, Zoladz PR, Conrad CD, Fleshner M, Diamond DM (2008) Acute predator stress impairs the consolidation and retrieval of hippocampus-dependent memory in male and female rats. Learn Mem 15:271-280 . https://doi.org/10.1101/lm.721108

Parker CE (1978) Opportunism and the Rise of Intelligence. J Hum Evol 7:597-608

Parker ST, Gibson KR (1977) Object manipulation, tool use and sensorimotor intelligence as feeding adaptations in cebus monkeys and great apes. J Hum Evol 6:623-641. https://doi.org/10.1016/S0047-2484(77)80135-8

Peckre L, Kappeler PM, Fichtel C (2019) Clarifying and expanding the social complexity hypothesis for communicative complexity. Behav Ecol Sociobiol 73:11 . https://doi.org/10.1007/s00265018-2605-4

Peichl L, Kaiser A, Rakotondraparany F, Dubielzig RR, Goodman SM, Kappeler PM (2019) Diversity of photoreceptor arrangements in nocturnal, cathemeral and diurnal Malagasy lemurs. J Comp Neurol 527:13-37 . https://doi.org/10.1002/cne.24167

Peres CA (1989) Exudate-eating by wild golden lion tamarins, Leontopithecus rosalia. Biotropica 21:287-288

Pérez-Barbería FJ, Shultz S, Dunbar RIM (2007) Evidence for coevolution of sociality and relative brain size in three orders of mammals. Evolution 61:2811-2821 . https://doi.org/10.1111/j.15585646.2007.00229.x

Perry SE, Barrett BJ, Godoy I (2017) Older, sociable capuchins (Cebus capucinus) invent more social behaviors, but younger monkeys innovate more in other contexts. P Natl Acad Sci USA 114:7806-7813 . https://doi.org/10.1073/pnas.1620739114

Picq JL (2007) Aging affects executive functions and memory in mouse lemur primates. Exp Gerontol 42:223-232 . https://doi.org/10.1016/j.exger.2006.09.013

Picq JL, Aujard F, Volk A, Dhenain M (2012) Age-related cerebral atrophy in nonhuman primates predicts cognitive impairments. Neurobiol Aging 33:1096-1109 . https://doi.org/10.1016/j.neurobiolaging.2010.09.009

Pike TW, Ramsey M, Wilkinson A (2018) Environmentally induced changes to brain morphology 
predict cognitive performance. Phil Trans R Soc B 373: . https://doi.org/10.1098/rstb.2017.0287 Pladevall J, Mendes N, Riba D, Llorente M, Amici F (2020) No evidence of what-where-when memory in great apes (Pan troglodytes, Pan paniscus, Pongo abelii, and Gorilla gorilla). J Comp Psychol 134:252-261 . https://doi.org/10.1037/com0000215

Platt ML, Brannon EM, Briese TL, French JA (1996) Differences in feeding ecology predict differences in performance between golden lion tamarins (Leontopithecus rosalia) and Wied's marmosets (Callithrix kuhli) on spatial and visual memory tasks. Anim Learn Behav 24:384393 . https://doi.org/10.3758/BF03199010

Pleskacheva MG, Nikolenko DL, Wolfer DP, Scheffrahn H, Dell'Omo G, Lipp H-PP, Kupriyanova IF, Nikolenko DL, Scheffrahn H, Dell'Omo G, Lipp H-PP (2000) Hippocampal mossy fibers and swimming navigation learning in two vole species occupying different habitats. Hippocampus 10:17-30 . https://doi.org/10.1002/(SICI)1098-1063(2000)10:1<17::AIDHIPO2>3.0.CO;2-O

Poirier M-A, Kozlovsky DY, Morand-Ferron J, Careau V (2020) How general is cognitive ability in non-human animals? A meta-analytical and multi-level reanalysis approach. Proc R Soc B 287:20201853 . https://doi.org/10.1098/rspb.2020.1853

Powell LE, Isler K, Barton RA (2017) Re-evaluating the link between brain size and behavioural ecology in primates. Proc R Soc B 284:20171765 . https://doi.org/10.1098/rspb.2017.1765

Pravosudov V V., De Kort SR (2005) Is the western scrub-jay (Aphelocoma californica) really an underdog among food-caching corvids when it comes to hippocampal volume and food caching propensity? Brain Behav Evol 67:1-9 . https://doi.org/10.1159/000088855

Preiszner B, Papp S, Pipoly I, Seress G, Vincze E, Liker A, Bókony V (2017) Problem-solving performance and reproductive success of great tits in urban and forest habitats. Anim Cogn 20:53-63 . https://doi.org/10.1007/s10071-016-1008-z

Prétôt L, Bshary R, Brosnan SF, Bshary R, Brosnan SF, Prétôt L, Bshary R, Brosnan SF (2016) Factors influencing the different performance of fish and primates on a dichotomous choice task. Anim Behav 119:189-199 . https://doi.org/10.1016/j.anbehav.2016.06.023

Pritchard DJ, Hurly TA, Tello-Ramos MC, Healy SD (2016) Why study cognition in the wild (and how to test it)? J Exp Anal Behav 105:41-55 . https://doi.org/10.1002/jeab.195

Rakotondranary SJ, Ganzhorn JU (2012) Habitat separation of sympatric microcebus spp. in the dry spiny forest of South-Eastern madagascar. Folia Primatol 82:212-223 . https://doi.org/10.1159/000334816

Rakotondravony R, Radespiel U (2009) Varying patterns of coexistence of two mouse lemur species (Microcebus ravelobensis and M. murinus) in a heterogeneous landscape. Am J Primatol 71:928938 . https://doi.org/10.1002/ajp.20732

Rakotoniaina JH, Kappeler PM, Ravoniarimbinina P, Pechouskova E, Hämäläinen AM, Grass J, Kirschbaum C, Kraus C (2016) Does habitat disturbance affect stress, body condition and parasitism in two sympatric lemurs? Conserv Physiol 4:cow034. https://doi.org/10.1093/conphys/cow034

Ramsey G, Bastian ML, van Schaik CP (2007) Animal innovation defined and operationalized. Behav Brain Sci 30:393-407; discussion 407-32 . https://doi.org/10.1017/S0140525X07002373

Rasoloarison RM, Goodman SM, Ganzhorn JU (2000) A taxonomic revision of mouse lemurs (Microcebus) occurring in the western portion of Madagascar. Int J Primatol 21:963-1019

Rasolofoniaina BN, Kappeler PM, Fichtel C (2020) Wild narrow-striped mongooses use social information to enhance behavioural flexibility. Ethology 127:1-14 . https://doi.org/10.1111/eth.13123

Ratcliffe JM, Fenton MB, Shettleworth SJ (2006) Behavioral flexibility positively correlated with relative brain volume in predatory bats. Brain Behav Evol 67:165-176 . https://doi.org/10.1159/000090980

Ratsimbazafy J, Clark C, Durbin J, Randriamahazo H, Copsey J, Rakotondravony D, Ramilijaona O (2016) Gestion des Éspèces Menacées: Cas des Vertébrés. In: Cullman G, Rakotobe D (eds) 


\section{References}

Lessons in Conservation. Center for Biodiversity and Conservation of the American Museum of Natural History, pp 62-79

Reader SM (2003) Innovation and social learning: individual variation and brain evolution. Anim Biol 53:147-158 . https://doi.org/10.1163/157075603769700340

Reader SM, Hager Y, Laland KN (2011) The evolution of primate general and cultural intelligence. Phil Trans R Soc B 366:1017-1027 . https://doi.org/10.1098/rstb.2010.0342

Reader SM, Laland KN (2002) Social intelligence, innovation, and enhanced brain size in primates. P Natl Acad Sci USA 99:4436-4441 . https://doi.org/10.1073/pnas.062041299

Reader SM, Laland KN (2003) Animal innovation. Oxford University Press

Reader SM, MacDonald K (2003) Environmental variability and primate behavioural flexibility. In: Reader SM, Laland KN (eds) Animal Innovation. Oxford University Press, Oxford, pp 83-116

Reader SM, Morand-Ferron J, Flynn E (2016) Animal and human innovation: novel problems and novel solutions. Phil Trans R Soc B 371:20150182 . https://doi.org/10.1098/rstb.2015.0182

Rendell L, Whitehead H (2001) Culture in whales and dolphins. Behav Brain Sci 24:309-324 . https://doi.org/10.1017/S0140525X0100396X

Reuter KE, Blanco M, Ganzhorn J, Schwitzer C (2020) Microcebus murinus (amended version of 2020 assessment). IUCN Red List Threat Species 2020 eT163314248A182239898. https://doi.org/10.2305/IUCN.UK.2020-3.RLTS.T163314248A182239898.en

Revelle W (2020) Procedures for psychological, psychometric, and personality research. CRAN Repos

Rico-Guevara A, Rubega MA, Hurme KJ, Dudley R (2019) Shifting paradigms in the mechanics of nectar extraction and hummingbird bill morphology. Integr Org Biol 1: . https://doi.org/10.1093/iob/oby006

Riley E, Lee R, Sangermano F, Cannon C, Shekelle M (2020) Macaca tonkeana. IUCN Red List Threat Species $2020 \quad$ eT12563A17947990. https://doi.org/10.2305/IUCN.UK.20203.RLTS.T12563A17947990.en

Roobas B, Feulner GR (2013) A population of Bosk's fringe-toed lizard Acanthodactylus boskianus (Daudin, 1802) in the Hajar Mountain foothills of the UAE. In: Tribulus. Emirates Natural History Group, pp 24-37

Rosati AG (2017a) Foraging cognition: reviving the ecological intelligence hypothesis. Trends Cogn Sci 21:691-702 . https://doi.org/10.1016/j.tics.2017.05.011

Rosati AG (2017b) The evolution of primate executive function : From response control to strategic decision-making. In: Evolution of Nervous System. Elsevier, pp 423-437

Rosati AG, Hare B (2012) Chimpanzees and bonobos exhibit divergent spatial memory development. Dev Sci 6:840-853 . https://doi.org/10.1111/j.1467-7687.2012.01182.x

Rosati AG, Rodriguez K, Hare B (2014) The ecology of spatial memory in four lemur species. Anim Cogn 17:947-961 . https://doi.org/10.1007/s10071-014-0727-2

Roth G, Dicke U (2005) Evolution of the brain and intelligence. Trends Cogn Sci 9:250-257 . https://doi.org/10.1016/j.tics.2005.03.005

Roth TC, Ladage LD, Pravosudov V V. (2010) Learning capabilities enhanced in harsh environments: a common garden approach. Proc R Soc B 277:3187-3193 . https://doi.org/10.1098/rspb.2010.0630

Roth TC, Pravosudov V V. (2009) Hippocampal volumes and neuron numbers increase along a gradient of environmental harshness: a large-scale comparison. Proc R Soc B 276:401-405 . https://doi.org/10.1098/rspb.2008.1184

Roughgarden J (1972) Evolution of niche width. Am Nat 106:683-718

Rowe C, Healy SD (2014) Measuring variation in cognition. Behav Ecol 25:1287-1292 . https://doi.org/10.1093/beheco/aru090

Rozin P (1976) Psychobiological and cultural determinants of food choice. In: Appetite and food intake: Report of the Dahlem Workshop. Abakon Verlagsgesellschaft, Oxford, England, England, p 499 
Rumbaugh DM, Bell CL, Gill T V. (1972) Two discrimination test apparatuses for primates. Behav Res Methods Instrum 4:6-10 . https://doi.org/10.3758/BF03209962

Rumbaugh DM, Gill T V. (1973) The learning skills of great apes. J Hum Evol 2:171-179 . https://doi.org/10.1016/0047-2484(73)90073-0

Rumbaugh DM, Pate JL (1984) Priamtes' learning by levels. In: Greenberg G, Tobach E (eds) Behavioral Evolution and Integrative Levels. pp 221-239

Rumbaugh DM, Washburn DA (2003) Intelligence of apes and other rational beings. Yale Universitay Press

Rylands AB (1989) Sympatric brazilian callitrichids: the black tufted-ear marmoset, Callithrix kuhli, and the golden-headed lion tamarin, Leontopithecus chrysomelas. J Hum Evol 18:679-695 . https://doi.org/10.1016/0047-2484(89)90100-0

Salwiczek LH, Prétôt L, Demarta L, Proctor D, Essler J, Pinto AI, Wismer S, Stoinski T, Brosnan SF, Bshary R, Salwiczek LH, Pre L, Wismer S, Stoinski T, Brosnan SF, Bshary R (2012) Adult cleaner wrasse outperform capuchin monkeys, chimpanzees and orang-utans in a complex foraging task derived from cleaner-client reef fish cooperation. PLoS ONE 7: . https://doi.org/10.1371/journal.pone.0049068

Santos LR, Mahajan N, Barnes JL (2005) How prosimian primates represent tools: experiments with two lemur species (Eulemur fulvus and Lemur catta). J Comp Psychol 119:394-403 . https://doi.org/10.1037/0735-7036.119.4.394

Sargeant BL (2007) Individual foraging specialization: niche width versus niche overlap. Oikos 116:1431-1437 . https://doi.org/10.1111/j.2007.0030-1299.15833.x

Sayol F, Maspons J, Lapiedra O, Iwaniuk AN, Székely T, Sol D (2016) Environmental variation and the evolution of large brains in birds. Nat Commun 7:13971 . https://doi.org/10.1038/ncomms13971

Schäffler L, Kappeler PM (2014) Distribution and abundance of the world's smallest primate, Microcebus berthae, in central western Madagascar. Int J Primatol 35:557-572 . https://doi.org/10.1007/s10764-014-9768-2

Schäffler L, Kappeler PM, Halley JM (2021) Mouse lemurs in an assemblage of cheirogaleid primates in Menabe central, western Madagascar - three reasons to coexist. Front Ecol Evol 9:1-17 . https://doi.org/10.3389/fevo.2021.585781

Schilling A (2012) Cognitive capacities of captive gray mouse lemurs as evidenced by object manipulation. In: Masters J, Gamba M, Génin F (eds) Leaping Ahead. Springer New York, New York, NY, pp 331-340

Schilling A (1979) Olfactory communication in prosimians. In: Doyle GA, Martin RD (eds) The study of prosimian behavior. Academic Press, London, pp 461-542

Schmid J (1999) Sex-specific differences in activity patterns and fattening in the gray mouse lemur (Microcebus murinus) in Madagascar. J Mammal 80:749-757 . https://doi.org/10.2307/1383244

Schmid J (1998) Tree holes used for resting by gray mouse lemurs (Microcebus murinus) in Madagascar: Insulation capacities and energetic consequences. Int J Primatol 19:797-809 . https://doi.org/10.1023/A:1020389228665

Schmid J, Kappeler PM (1994) Sympatric mmouse lemurs (Microcebus spp.) in western Madagascar. Folia Primatol 63:162-170 . https://doi.org/10.1159/000156812

Schmid J, Ruf T, Heldmaier G (2000) Metabolism and temperature regulation during daily torpor in the smallest primate, the pygmy mouse lemur (Microcebus myoxinus) in Madagascar. J Comp Physiol B-Biochemical Syst Environ Physiol 170:59-68. https://doi.org/10.1007/s003600050008

Schmidtke D, Ammersdörfer S, Joly M, Zimmermann E (2018) First comparative approach to touchscreen-based visual object-location paired-associates learning in humans (Homo sapiens) and a nonhuman primate (Microcebus murinus). J Comp Psychol 132:315-325 . https://doi.org/10.1037/com0000116

Schnitzler HU, Moss CF, Denzinger A (2003) From spatial orientation to food acquisition in 


\section{References}

echolocating bats. Trends Ecol Evol 18:386-394 . https://doi.org/10.1016/S0169-5347(03)00185$\mathrm{X}$

Schradin C, Pillay N (2004) The striped mouse (Rhabdomys pumilio) from the succulent Karoo, South Africa: A territorial group-living solitary forager with communal breeding and helpers at the nest. J Comp Psychol 118:37-47 . https://doi.org/10.1037/0735-7036.118.1.37

Schubiger MN, Fichtel C, Burkart JM (2020) Validity of cognitive tests for non-human animals: Pitfalls and prospects. Front Psychol 11:1835 . https://doi.org/10.3389/fpsyg.2020.01835

Schuck-Paim C, Alonso WJ, Ottoni EB, Ottoni B, Ottoni EB (2008) Cognition in an ever-changing world: Climatic variability is associated with brain size in neotropical parrots. Brain Behav Evol 71:200-215 . https://doi.org/10.1159/000119710

Schülke O, Ostner J (2007) Physiological ecology of cheirogaleid primates: Variation in hibernation and torpor. Acta Ethol 10:13-21 . https://doi.org/10.1007/s10211-006-0023-5

Schüßler D, Blanco MB, Salmona J, Poelstra J, Andriambeloson JB, Miller A, Randrianambinina B, Rasolofoson DW, Mantilla-Contreras J, Chikhi L, Louis EE, Yoder AD, Radespiel U (2020) Ecology and morphology of mouse lemurs (Microcebus spp.) in a hotspot of microendemism in northeastern Madagascar, with the description of a new species. Am J Primatol 82:1-18 . https://doi.org/10.1002/ajp.23180

Schwab D, Ganzhorn JU (2004) Distribution, population structure and habitat use of Microcebus berthae compared to those of other sympatric cheirogaleids. Int J Primatol 25:307-330 . https://doi.org/10.1023/B:IJOP.0000019154.17401.90

Schwitzer C, Mittermeier RA, Johnson SE, Donati G, Irwin M, Peacock H, Ratsimbazafy J, Razafindramanana J, Louis EE, Chikhi L, Colquhoun IC, Tinsman J, Dolch R, LaFleur M, Nash S, Patel E, Randrianambinina B, Rasolofoharivelo T, Wright PC (2014a) Averting lemur extinctions amid Madagascar's political crisis. Science 343:842-843. https://doi.org/10.1126/science.1245783

Schwitzer C, Mittermeier RA, Rylands AB, Taylor LA, Chiozza F, Williamson EA, Wallis J, Clark FE (2014b) Primates in Peril. IUCN SSC Primate Specialist Group (PSG), International Primatological Society (IPS), Conservation International (CI), Bristol Zoological Society (BZS)

Shaw RC, Boogert NJ, Clayton NS, Burns KC (2015) Wild psychometrics: evidence for 'general' cognitive performance in wild New Zealand robins, Petroica longipes. Anim Behav 109:101111 . https://doi.org/10.1016/j.anbehav.2015.08.001

Shaw RC, MacKinlay RD, Clayton NS, Burns KC (2019) Memory performance influences male reproductive success in a wild bird. Curr Biol 29:1498-1502.e3 . https://doi.org/10.1016/j.cub.2019.03.027

Shaw RC, Schmelz M (2017) Cognitive test batteries in animal cognition research: evaluating the past, present and future of comparative psychometrics. Anim Cogn 20:1003-1018 . https://doi.org/10.1007/s10071-017-1135-1

Sherry DF, Jacobs LF, Gaulin SJC (1992) Spatial memory and adaptive specialization of the hippocampus. Trends Neurosci 15:298-303 . https://doi.org/10.1016/0166-2236(92)90080-R

Shettleworth SJ (2009) Cognition, evolution, and behavior, 2nd edn. Oxford University Press, Oxford

Shettleworth SJ (1990) Spatial memory in food-storing birds. Phil Trans R Soc Lond Ser B 329:143151 . https://doi.org/10.1098/rstb.1990.0159

Shettleworth SJ (2001) Animal cognition and animal behaviour. Anim Behav 61:277-286 . https://doi.org/10.1006/anbe.2000.1606

Shettleworth SJ, Krebs JR, Healy SD, Thomas CM (1990) Spatial memory of food-storing tits (Parus ater and P. atricapillus) : comparison of storing and nonstoring tasks. J Comp Psychol 104:7181 . https://doi.org/10.1037/0735-7036.104.1.71

Shultz S, Dunbar RIM (2006) Both social and ecological factors predict ungulate brain size. Proc R Soc B 273:207-215 . https://doi.org/10.1098/rspb.2005.3283

Shultz S, Dunbar RIM (2007) The evolution of the social brain: Anthropoid primates contrast with 
other vertebrates. Proc R Soc B 274:2429-2436 . https://doi.org/10.1098/rspb.2007.0693

Shumway CA (2008) Habitat complexity, brain, and behavior. Brain Behav Evol 72:123-134 . https://doi.org/10.1159/000151472

Siemers BM, Goerlitz HR, Robsomanitrandrasana E, Piep M, Ramanamanjato JB, Rakotondravony D, Ramilijaona O, Ganzhorn JU (2007) Sensory basis of food detection in wild Microcebus murinus. Int J Primatol 28:291-304 . https://doi.org/10.1007/s10764-007-9135-7

Sih A (2013) Understanding variation in behavioural responses to human-induced rapid environmental change: A conceptual overview. Anim Behav 85:1077-1088 . https://doi.org/10.1016/j.anbehav.2013.02.017

Sih A, Bell A, Johnson JC (2004) Behavioral syndromes: An ecological and evolutionary overview. Trends Ecol Evol 19:372-378 . https://doi.org/10.1016/j.tree.2004.04.009

Sol D, Bacher S, Reader SM, Lefebvre L (2008) Brain size predicts the success of mammal species introduced into novel environments. Am Nat 172:S63-S71 . https://doi.org/10.1086/588304

Sol D, Duncan RP, Blackburn TM, Cassey P, Lefebvre L (2005a) Big brains, enhanced cognition, and response of birds to novel environments. P Natl Acad Sci USA 102:5460-5465 . https://doi.org/10.1073/pnas.0408145102

Sol D, Griffin AS, Bartomeus I (2012) Consumer and motor innovation in the common myna: the role of motivation and emotional responses. Anim Behav 83:179-188 . https://doi.org/10.1016/j.anbehav.2011.10.024

Sol D, Lapiedra O, González-Lagos C (2013) Behavioural adjustments for a life in the city. Anim Behav 85:1101-1112 . https://doi.org/10.1016/j.anbehav.2013.01.023

Sol D, Lefebvre L (2000) Behavioural flexibility predicts invasion success in birds introduced to New Zealand. Oikos 90:599-605 . https://doi.org/10.1034/j.1600-0706.2000.900317.x

Sol D, Lefebvre L, Rodríguez-Teijeiro JD (2005b) Brain size, innovative propensity and migratory behaviour in temperate Palaearctic birds. Proc R Soc B 272:1433-1441. https://doi.org/10.1098/rspb.2005.3099

Sol D, Stirling DG, Lefebvre L (2005c) Behavioral drive or behavioral inhibition in evolution: Subspecific diversification in holarctic passerines. Evolution 59:2669 . https://doi.org/10.1554/05-196.1

Sol D, Timmermans S, Lefebvre L (2002) Behavioural flexibility and invasion success in birds. Anim Behav 63:495-502 . https://doi.org/10.1006/anbe.2001.1953

Soler JJ, Peralta-Sánchez JM, Martín-Vivaldi M, Martín-Platero AM, Flensted-Jensen E, Møller AP (2012) Cognitive skills and bacterial load: comparative evidence of costs of cognitive proficiency in birds. Naturwissenschaften 99:111-122 . https://doi.org/10.1007/s00114-0110875-z

Sonnenberg BR, Branch CL, Pitera AM, Bridge E, Pravosudov V V., Sonnenberg BR, Branch CL, Pitera AM, Bridge E, Pravosudov V V. (2019) Natural selection and spatial cognition in wild food-caching mountain chickadees. Curr Biol 29:670-676.e3 . https://doi.org/10.1016/j.cub.2019.01.006

Spearman C (1904) "General intelligence" objectively determined and measured. Am J Psychol 15:201-292

Stevens JR, Hallinan E V., Hauser MD (2005) The ecology and evolution of patience in two New World monkeys. Biol Lett 1:223-226 . https://doi.org/10.1098/rsbl.2004.0285

Street SE, Navarrete AF, Reader SM, Laland KN (2017) Coevolution of cultural intelligence, extended life history, sociality, and brain size in primates. P Natl Acad Sci USA 114:7908-7914 . https://doi.org/10.1073/pnas.1620734114

Striedter GF (2006) Précis of principles of brain evolution. Behav Brain Sci 29:1-12 . https://doi.org/10.1017/S0140525X06009010

Szabo B, Whiting MJ (2020) Do lizards have enhanced inhibition? A test in two species differing in ecology and sociobiology. Behav Process 172:104043 https://doi.org/10.1016/j.beproc.2020.104043 


\section{References}

Tebbich S, Griffin AS, Peschl MF, Sterelny K (2016) From mechanisms to function: an integrated framework of animal innovation. Phil Trans R Soc Lond B 371:20150195- . https://doi.org/10.1098/rstb.2015.0195

Tebbich S, Stankewitz S, Teschke I (2012) The relationship between foraging, learning abilities and neophobia in two species of Darwin's finches. Ethology 118:135-146. https://doi.org/10.1111/j.1439-0310.2011.02001.x

Tebbich S, Teschke I (2014) Coping with uncertainty: Woodpecker finches (Cactospiza pallida) from an unpredictable habitat are more flexible than birds from a stable habitat. PLoS ONE 9:e91718 . https://doi.org/10.1371/journal.pone.0091718

Teichroeb JA, Vining AQ (2019) Navigation strategies in three nocturnal lemur species: diet predicts heuristic use and degree of exploratory behavior. Anim Cogn 22:343-354 . https://doi.org/10.1007/s10071-019-01247-4

Temeles EJ, Linhart YB, Masonjones M, Masonjones HD (2002) The role of flower width in hummingbird bill length - Flower length relationships. Biotropica 34:68-80 . https://doi.org/10.1111/j.1744-7429.2002.tb00243.x

Therneau T, Lumley T, Atkinson E, Crowson C (2020) Survival analysis. CRAN Repos

Thorndike EL (1898) Animal intelligence: An experimental study of the associative processes in animals. Am Psychol 53:551-553

Thornton A (2014) How and why are some species so smart? A comment on Rowe and Healy. Behav Ecol 25:1294-1295 . https://doi.org/10.1093/beheco/aru115

Thornton A, Isden J, Madden JR (2014) Toward wild psychometrics: Linking individual cognitive differences to fitness. Behav Ecol 25:1299-1301 . https://doi.org/10.1093/beheco/aru095

Thornton A, Lukas D (2012) Individual variation in cognitive performance: developmental and evolutionary perspectives. Phil Trans R Soc B 367:2773-2783. https://doi.org/10.1098/rstb.2012.0214

Thornton A, Samson J (2012) Innovative problem solving in wild meerkats. Anim Behav 83:14591468 . https://doi.org/10.1016/j.anbehav.2012.03.018

Timm R, Álvarez-Castaeda ST, Lacher T (2016) Dipodomys merriami. In: IUCN Red List Threat Species 2016eT92465716A115515430

Todorov OS, Weisbecker V, Gilissen E, Zilles K, De Sousa AA, Sousa AA De (2019) Primate hippocampus size and organization are predicted by sociality but not diet. Proc R Soc B 286: . https://doi.org/10.1098/rspb.2019.1712

Tooby J, Cosmides L (2003) The psychological foundations of culture. Psychology Press, New York Trapanese C, Robira B, Tonachella G, di Gristina S, Meunier H, Masi S (2019) Where and what? Frugivory is associated with more efficient foraging in three semi-free ranging primate species. R Soc Open Sci 6:181722 . https://doi.org/10.1098/rsos.181722

Trouche SG, Maurice T, Rouland S, Verdier JM, Mestre-Francés N (2010) The three-panel runway maze adapted to Microcebus murinus reveals age-related differences in memory and perseverance performances. Neurobiol Learn Mem 94:100-106 . https://doi.org/10.1016/j.nlm.2010.04.006

van Horik JO, Madden JR (2016) A problem with problem solving: Motivational traits, but not cognition, predict success on novel operant foraging tasks. Anim Behav 114:189-198 . https://doi.org/10.1016/j.anbehav.2016.02.006

van Schaik CP, Burkart JM (2011) Social learning and evolution: the cultural intelligence hypothesis. Phil Trans R Soc B 366:1008-1016 . https://doi.org/10.1098/rstb.2010.0304

van Woerden JT, Willems EP, van Schaik CP, Isler K (2012) Large brains buffer energetic effects of seasonal habitats in catarrhine primates. Evolution 66:191-199 . https://doi.org/10.1111/j.15585646.2011.01434.x

Vas Z, Lefebvre L, Johnson KP, Reiczigel J, Rózsa L (2011) Clever birds are lousy: Co-variation between avian innovation and the taxonomic richness of their amblyceran lice. Int J Parasitol 41:1295-1300 . https://doi.org/10.1016/j.ijpara.2011.07.011 
Verdolin JL, Harper J (2013) Are shy individuals less behaviorally variable? Insights from a captive population of mouse lemurs. Primates 54:309-314 . https://doi.org/10.1007/s10329-013-0360-8

Vitale AF, Visalberghi E, De Lillo C (1990) Responses to a model snake in captive crab-eating macaques (Macaca fascicularis) and tufted capuchins (Cebus apella): A comparison. Ethol Ecol Evol 2:333-334 . https://doi.org/10.1080/08927014.1990.9525479

Völter CJ, Tinklenberg B, Call J, Seed AM (2018) Comparative psychometrics: establishing what differs is central to understanding what evolves. Phil Trans R Soc B 373:20170283 . https://doi.org/10.1098/rstb.2017.0283

Wall V, Stephen B (1990) Food hoarding in animals. University of Chicago Press, Chicago

Wetzel DP (2017) Problem-solving skills are linked to parental care and offspring survival in wild house sparrows. Ethology 123:475-483 . https://doi.org/10.1111/eth.12618

White GE, Brown C (2015a) Cue choice and spatial learning ability are affected by habitat complexity in intertidal gobies. Behav Ecol 26:178-184 . https://doi.org/10.1093/beheco/aru178

White GE, Brown C (2015b) Microhabitat use affects brain size and structure in intertidal gobies. Brain Behav Evol 85:107-116 . https://doi.org/10.1159/000380875

Whiten A (2017) Culture extends the scope of evolutionary biology in the great apes. P Natl Acad Sci USA 114:7790-7797 . https://doi.org/10.1073/pnas.1620733114

Whiten A (2000) Primate culture and social learning. Cogn Sci 24:477-508 . https://doi.org/10.1207/s15516709cog2403_6

Whiten A, Byrne RW (1988) Machiavellian intelligence: Social expertise and the evolution of intellect in monkeys, apes, and humans. Oxford University Press, New York

Whiten A, van Schaik CP (2007) The evolution of animal "cultures" and social intelligence. Phil Trans R Soc B 362:603-620 . https://doi.org/10.1098/rstb.2006.1998

Wild K, Howieson D, Webbe F, Seelye A, Kaye J (2008) Status of computerized cognitive testing in aging: A systematic review. Alzheimer's Dement 4:428-437. https://doi.org/10.1016/j.jalz.2008.07.003

Wilke A, Hutchinson JMCC, Todd PM, Czienskowski U (2009) Fishing for the right words: Decision rules for human foraging behavior in internal search tasks. Cogn Sci 33:497-529 . https://doi.org/10.1111/j.1551-6709.2009.01020.x

Wilmé L, Goodman S, Ganzhorn J (2006) Biogeographic evolution of Madagascar's microendemic biota. Science 312:1063-1065

Wilson DS, Yoshimura J (2008) On the coexistence of specialists and generalists. Am Nat 144:692707

Wither RG, Boehnke SE, Lablans A, Armitage-Brown B, Munoz DP (2020) Behavioral shaping of rhesus macaques using the Cambridge neuropsychological automated testing battery. J Neurosci Methods 342:108803 . https://doi.org/10.1016/j.jneumeth.2020.108803

Wolak M (2015) Facilitating estimation of the intraclass correlation coefficient. CRAN Repos 1-9

Woodley of Menie MA, Fernandes HBF, Hopkins WD (2015) The more g-loaded, the more heritable, evolvable, and phenotypically variable: Homology with humans in chimpanzee cognitive abilities. Intelligence 50:159-163 . https://doi.org/10.1016/j.intell.2015.04.002

Wright TF, Eberhard JRR, Hobson E a. A, Avery MLL, Russello M a. A (2010) Behavioral flexibility and species invasions: The adaptive flexibility hypothesis. Ethol Ecol Evol 22:393-404 . https://doi.org/10.1080/03949370.2010.505580

Yoder AD, Campbell CR, Blanco MB, Dos Reis M, Ganzhorn JU, Goodman SM, Hunnicutt KE, Larsen PA, Kappeler PM, Rasoloarison RM, Ralison JM, Swofford DL, Weisrock DW (2016) Geogenetic patterns in mouse lemurs (genus $<\mathrm{i}>$ Microcebus $<\mathrm{i} />$ ) reveal the ghosts of Madagascar's forests past. P Natl Acad Sci USA 113:8049-8056 . https://doi.org/10.1073/pnas.1601081113

Yoder AD, Rasoloarison RM, Goodman SM, Irwin JA, Atsalis S, Ravosa MJ, Ganzhorn JU (2000) Remarkable species diversity in Malagasy mouse lemurs (primates, Microcebus). P Natl Acad Sci USA 97:11325-30 . https://doi.org/10.1073/pnas.200121897 


\section{References}

Zimmermann E, Radespiel U (2014) Species concepts, diversity, and evolution in primates: Lessons to be learned from mouse lemurs. Evol Anthropol 23:11-14 https://doi.org/10.1002/evan.21388 


\section{Acknowledgements}

To plan, execute and finalize a research project at the scale of my thesis that constitutes a combination of various experimental studies applied to a large sample size of wild animals, it requires a lot of physical assistance, but also mental support. Many scholars and non-scholars supported me and my work in the past four years.

Essentially, Dr. Claudia Fichtel and Prof. Peter M. Kappeler gave me the chance to develop and work on this exciting project. I want to express my sincere appreciation for their academic supervision, their constructive feedback, and their faith in my professional working attitude that helped to improve my research, as well as my scientific skills.

I am thankful to Prof. Julia Ostner and Prof. Lars Penke for their critical ideas and suggestions on my project in the scope of our thesis committee meetings, and for their realistic estimation of a healthy workload. My deep gratitude goes to Prof. Stefan Scheu, who, as my mentor within the Dorothea Schlözer mentoring program, helped me to overcome periods of struggles, to stay motivated, and to regain resilience which ultimately led me to finalize my dissertation. I want to further thank Prof. Dr. Katja Liebal for complementing my examination board.

In Madagascar, I received major assistance of the Equipe Kirindy, most importantly from Bruno Tsiverimanana and his unfailing commitment to co-work with me at any occurring tasks which stand in relation to the mouse lemurs. Nandeha haingana ny fotoana - ny fotoana tena nahafinaritra niaraka taminao, niaraka amin'ny asa mafy be sy ny mozika be dia be. Misaotra betsaka daholo e, nanao ny telo taona ho zavatra tsy hay hadinoina ho ahy! Mandra-pihaona sy ho ela velona!

Also, a huge thank you to my highly motivated student volunteers - Jan Förster, Joe Buchner, Luca Hahn, Svenja Blödorn, Joana Niedner, Colleen Illing, Annkatrin Pahl, Frederike Hoppmann, and Tímea Kovács - for their help with the data collection and video analyses. I also want to thank Katja Rudolph, Colleen Illing, Bako N. Rasolofoniaina, and Lea Prox for helpful comments on parts of my thesis, and all the past and present members of the Behavioral Ecology and Sociobiology Unit of the German Primate Center for fruitful scientific discussions. Thank you Mamy S. Razafindrasamba for allowing to 


\section{Acknowledgements}

include one of your incredible drawings into my thesis, and Katja Rudolph for providing the adorable mouse lemur icons.

Finally, most of the mental support came from my family, my friends, and my colleagues in Madagascar and Germany. Thank you, Mama, Papa, Katja, Colleen, Flo and all the others for your confidence and support during various periods and stages of my studies. 


\title{
Curriculum Vitae
}

\author{
Personal information \\ Johanna Henke-von der Malsburg \\ Born May 31st, 1993 in Gräfelfing (Germany) \\ Nationality: German
}

\section{Scientific education and research experience}

$04 / 2017-05 / 2021$

$12 / 2016-05 / 2017$

$10 / 2014-01 / 2017$

$10 / 2011-09 / 2014$

\section{Ph. D. in Behavior and Cognition}

Behavioral Ecology and Sociobiology Unit, German Primate Center \& Georg-August-University Göttingen, Germany

Project: Cognition in two sympatric mouse lemur species, the Grey mouse lemur (M. murinus) and the Madame Berthe's mouse lemur (M. berthae)

03/2017 - 11/2019 (1.5 years) Field experience: data collection, Kirindy forest, Morondava (Madagascar)

2020 Mentee in the Dorothea Schlözer mentoring program of the Georg-August-University Göttingen, Germany

Research assistant: Statistic analyses, experiments on cognition in lemurs

Behavioral Ecology and Sociobiology Unit, German Primate Center

M. Sc. Developmental, Neural and Behavioral Biology

Georg-August-University Göttingen, Germany

Thesis: Innovation in two sympatric mouse lemurs (M. murinus and M. berthae)

09/2015 - 10/2015 Field experience: lab course, Kirindy forest, Morondava (Madagascar)

08/2016 - 10/2016 Field experience: data collection for master's thesis, Kirindy forest, Morondava (Madagascar)

\section{B. Sc. Biology}

University of Hamburg, Germany

Thesis: Populationsgenetik des Graubraunen Mausmakis (M. griseorufus) im Nationalpark Tsimanampetsotsa, Madagaskar [Population genetics of the grey-brown mouse lemur (M. griseorufus) in the national park Tsimanampetsotsa, Madagascar]

09/2013 - 02/2014 Study abroad: Grado Biología at Universidad de La Laguna, San Cristóbal de La Laguna (Tenerife, Spain) 


\section{Scientific grants and awards}

2019 Diverse Intelligence Summer Institute

Templeton World Charity Foundation, St Andrews, Scotland, June 30 - July 20

2019 GfP-Award for best poster

$16^{\text {th }}$ Conference of the Gesellschaft für Primatologie e.V., Göttingen (Germany), February 13-15

2017 GfP-Award for master's Thesis

Gesellschaft für Primatologie e.V.

2016 PROMOS: Master's Thesis in Kirindy Forest (Madagascar)

Data collection of cognitive experiments on wild living mouse lemurs

2015 PROMOS: lab course in Kirindy Forest (Madagascar) within M. Sc.

Project: Reversal learning in two sympatric mouse lemurs (Microcebus murinus and M. berthae)

2013 ERASMUS: study abroad at Universidad de La Laguna (San Cristóbal de La Laguna, Tenerife, Spain): Grado Biología

\section{Publications and conference contributions}

In prep Article: Henke-von der Malsburg J., Fichtel C. Memory retention in relation to differential activity patterns over a hibernation period in gray mouse lemurs.

In prep Article: Henke-von der Malsburg J., Fichtel C. On the investigation of interspecific variation in cognition and the potential link with ecological factors.

2020 Article: Henke-von der Malsburg J., Kappeler P. M., Fichtel C. Linking ecology and cognition: does ecological specialization predict cognitive test performance?

Behavioral Ecology and Sociobiology, 74:154. DOI 10.1007/s00265-020-02923-z

2019 Presentation: Innovation in two sympatric mouse lemurs (Microcebus murinus, M. berthae)

$16^{\text {th }}$ Conference of the Gesellschaft für Primatologie e.V., Göttingen, February 13-15

2019 Poster: Do generalists and specialists differ in cognitive abilities? Award for best poster

$16^{\text {th }}$ Conference of the Gesellschaft für Primatologie e.V., Göttingen, February 13-15

2018 Article: Henke-von der Malsburg J., Fichtel C. Are generalists more innovative than specialists? A comparison of innovative abilities in two wild sympatric mouse lemur species.

Royal Society Open Science, 4, 180-480. DOI 10.1098/rsos.180480

2017 Poster: Behavioral Flexibility in problem-solving abilities in a generalist and specialist species, the grey mouse lemur and Madame Berthe's mouse lemur.

$50^{\text {th }}$ Anniversary of the Primatological Society of Great Britain, London (UK), November 28-29 
2017 Poster: Duel of mouse lemurs: Comparing innovative abilities in two sympatric mouse lemur species (Microcebus murinus \& Microcebus berthae).

$12^{\text {th }}$ Conference of the Ethologische Gesellschaft e.V., Bonn (Germany), February 22-24

2017 Poster: Innovation in two sympatric mouse lemur species (Microcebus murinus \& M. berthae).

$15^{\text {th }}$ Conference of the Gesellschaft für Primatologie e.V., Zurich (Switzerland), February 15-17

2015 Article: Scheel B. M., Henke-von der Malsburg J., Giertz P., Rakotondranary S. J., Hausdorf B., \& Ganzhorn J. U. Testing the influence of habitat structure and geographic distance on the genetic differentiation of mouse lemurs (Microcebus) in Madagascar.

International Journal of Primatology, 36(4), 823-838. DOI 10.1007/s10764-015-9855-z

2015 Poster: Population genetics of the reddish-grey mouse lemur (Microcebus griseorufus) in Tsimanampetsotsa National Park, Madagascar.

$14^{\text {th }}$ Conference of the Gesellschaft für Primatologie e.V., Leipzig (Germany), February 25-27 


\section{Declaration}

I hereby declare that all parts of my thesis titled "Cognitive adaptations in two sympatric mouse lemur species occupying different ecological niches" were written by myself. Assistance of third parties was only accepted if scientifically justifiable and acceptable in regard to the examination regulations. Assistance or contributions to the individual chapters are indicated and all sources have been quoted.

Göttingen, 21.04.2021

Johanna Henke-von der Malsburg 